\title{
Statistical Physics of Vehicular Traffic and Some Related Systems ${ }^{1}$
}

\author{
Debashish Chowdhury ${ }^{\mathrm{a}, 2}$ Ludger Santen ${ }^{\mathrm{a}, 3}$ \\ Andreas Schadschneider ${ }^{\text {a, }} 4$
}

${ }^{a}$ Institut für Theoretische Physik, Universität zu Köln, D-50923 Köln, Germany

Physics Reports 329, 199 (2000)

\begin{abstract}
In the so-called "microscopic" models of vehicular traffic, attention is paid explicitly to each individual vehicle each of which is represented by a "particle"; the nature of the "interactions" among these particles is determined by the way the vehicles influence each others' movement. Therefore, vehicular traffic, modeled as a system of interacting "particles" driven far from equilibrium, offers the possibility to study various fundamental aspects of truly nonequilibrium systems which are of current interest in statistical physics. Analytical as well as numerical techniques of statistical physics are being used to study these models to understand rich variety of physical phenomena exhibited by vehicular traffic. Some of these phenomena, observed in vehicular traffic under different circumstances, include transitions from one dynamical phase to another, criticality and self-organized criticality, metastability and hysteresis, phase-segregation, etc. In this critical review, written from the perspective of statistical physics, we explain the guiding principles behind all the main theoretical approaches. But we present detailed discussions on the results obtained mainly from the so-called "particle-hopping" models, particularly emphasizing those which have been formulated in recent years using the language of cellular automata.
\end{abstract}

Key words: Cellular automata, complex systems, nonequilibrium physics 


\section{Contents}

$\begin{array}{lll}1 & \text { Introduction } & 6\end{array}$

$2 \quad$ Fundamental and practical questions $\quad 9$

$\begin{array}{lll}2.1 & \text { Some fundamental questions } & 10\end{array}$

$\begin{array}{lll}2.2 & \text { Some practical questions } & 11\end{array}$

$3 \quad$ Some empirical facts and phenomenological explanations 12

$\begin{array}{llr}3.1 & \text { Acceleration noise } & 12\end{array}$

$\begin{array}{lll}3.2 & \text { Formation and characterization of traffic jams } & 12\end{array}$

\begin{tabular}{|lll}
\hline 3.3 & Flux-density relation & 14
\end{tabular}

\begin{tabular}{|lll}
3.4 & Microscopic states of traffic flow and phase transitions & 17
\end{tabular}

\begin{tabular}{lll}
\hline 3.5 & Time- and distance-headways & 18
\end{tabular}

$4 \quad$ Fluid-dynamical Theories of vehicular traffic 20

4.1 Lighthill-Whitham theory and kinematic waves 21

4.2 Diffusion term in Lighthill-Whitham theory and its effects 24

4.3 Greenshields model and Burgers equation 24

$4.4 \quad$ Navier-Stokes-like momentum equation and consequences $\quad 25$

$4.5 \quad$ Fluid-dynamical theories for multi-lane highways and city traffic 26

4.6 $\quad$ Some recent results of the fluid-dynamical theories and their physical $\begin{array}{ll}\text { implications } & 27\end{array}$

$\begin{array}{lll}5 & \text { Kinetic theories of vehicular traffic } & 29\end{array}$

$\begin{array}{lll}5.1 & \text { Prigogine model } & 30\end{array}$

$\begin{array}{lll}5.2 & \text { Paveri-Fontana model } & 31\end{array}$

1 Partially supported by SFB 341 (Köln-Aachen-Jülich)

2 On leave from Physics Department, I.I.T., Kanpur 208016, India; E-mail: debch@iitk.ac.in

3 present address: CNRS-Laboratoire de Physique Statistique, Ecole Normale Supérieure, 24, rue Lhomond, 75231 Paris Cedex 05, France ; E-mail: Ludger.Santen@lps.ens.fr

4 E-mail: as@thp.uni-koeln.de 
5.3 Derivation of the phenomenological equations of the macroscopid fluid-dynamical theories from the microscopic gas-kinetic models

$6 \quad$ Car-following theories of vehicular traffid 36

\begin{tabular}{|lll}
\hline 6.1 & Follow-the-leader model & 36
\end{tabular}

$\begin{array}{lll}6.2 & \text { Optimal velocity models } & 38\end{array}$

$7 \quad$ Coupled-map lattice models of vehicular traffic 42

$7.1 \quad$ The model of Yukawa and Kikuchi 43

\begin{tabular}{lll}
\hline 7.2 & The model of Nagel and Herrmann & 45
\end{tabular}

7.3 The model of Krauss, Wagner and Gawron 45

$8 \quad$ Nagel-Schreckenberg cellular automata model of vehicular traffic on $\begin{array}{ll}\text { highways } & 48\end{array}$

\begin{tabular}{lll}
\hline 8.1 & Relation with other models & 51
\end{tabular}

$\begin{array}{lll}8.2 & \text { Limiting cases of the NaSch mode } & 53\end{array}$

8.3 Analytical theories of the NaSch model with periodic boundary conditions 55

8.4 Spatio-temporal organization of vehicles; is there a phase transition? $\quad 66$

8.5 Exact solution of the NaSch model with $v_{\max }=1$ and open boundary conditions 76

$9 \quad$ Generalizations and extensions of the NaSch mode 81

$\begin{array}{lll}9.1 & \text { Single-lane highways } & 81\end{array}$

$\begin{array}{lll}9.2 & \text { Multi-lane highways } & 95\end{array}$

$\begin{array}{lll}9.3 & \text { Bidirectional traffic } & 98\end{array}$

$\begin{array}{lll}10 & \text { Effects of quenched disorder on traffic } & 100\end{array}$

10.1 Randomness in the braking probability of drivers and Bose-Einstein$\begin{array}{ll}\text { like condensation } & 100\end{array}$

$\begin{array}{ll}10.2 \text { Random } v_{\max } & 103\end{array}$

10.3 Randomly placed bottlenecks on the roads and the maximum flux principle 
$\begin{array}{lll}11 & \text { Other CA models of highway traffic } & 110\end{array}$

$\begin{array}{lll}11.1 & \text { Fukui-Ishibashi model } & 110\end{array}$

11.2 Galilei-invariant vehicle-vehicle interaction and metastability 111



$\begin{array}{ll}11.4 \text { CA from ultra-discretization } & 117\end{array}$

$\begin{array}{lll}12 & \text { Cellular automata models of city traffic and road networks } & 118\end{array}$

\begin{tabular}{|lll}
\hline 12.1 & Biham-Middleton-Levine model and its generalizations & 119
\end{tabular}

12.2 Marriage of the NaSch model and the BML model; a "unified" CA $\begin{array}{ll}\text { model of city traffid } & 127\end{array}$

12.3 Practical applications of the models of vehicular traffic; On-line simulation of traffic networks 131

$13 \quad$ Some related systems, models and phenomena 132



$\begin{array}{ll}13.2 \text { Surface growth, KPZ equation and Bethe Ansatz } & 134\end{array}$

$\begin{array}{ll}13.3 \text { Protein synthesis } & 135\end{array}$

$\begin{array}{ll}13.4 \text { Granular flow } & 136\end{array}$

$\begin{array}{ll}13.5 \text { The bus route model } & 136\end{array}$

\begin{tabular}{ll}
\hline 13.6 Mobile directional impurities & 138
\end{tabular}

$\begin{array}{lr}13.7 \text { Computer networks } & 139\end{array}$

\begin{tabular}{llr}
\hline 14 & Summary and conclusion & 140
\end{tabular}

\begin{tabular}{|ll}
\hline A $\quad$ Definition of update orders & 144
\end{tabular}

\begin{tabular}{lll}
\hline B TASEP & 145
\end{tabular}

$\begin{array}{lll}\text { C Naive site-oriented mean-field treatment of the NaSch mode } & 146\end{array}$

\begin{tabular}{lll}
\hline D & Paradisical mean-field theory & 147
\end{tabular}

E Equations of car-oriented theory of NaSch Model and COMF approximation 150

\begin{tabular}{lll}
\hline F The matrix-product Ansatz for stochastic systems & 150
\end{tabular} 
G Two schemes for solving the mean-field approximation of the DTASEP

H Self-consistent equations for $v_{x}$ and $v_{y}$ in the mean-field approximation of the BML model

I Derivation of the equations in the microscopic theory of the BMD model

References

156 


\section{Introduction}

The concepts and techniques of statistical physics are being used nowadays to study several aspects of complex systems [1] many of which, till a few decades ago, used to fall outside the traditional domain of physical systems [2]. Physical-, chemical-, earth-, biological- and social-sciences as well as technology meet at this frontier area of inter-disciplinary research. Flow of vehicular traffic and granular matter [3,4], folding of proteins [5], formation and growth of bacterial colonies [6], biological evolution of species [7] and transactions in financial markets [8] are just a few examples of exotic phenomena in such systems. Most of these systems are interesting not only from the point of view of Natural Sciences for fundamental understanding of how Nature works but also from the points of view of applied sciences and engineering for the potential practical use of the results of the investigations. Our review of the current status and future trends of research on the theory of vehicular traffic (and some related systems) will, we hope, convince you that, indeed, the results of recent studies of complex systems have been "a conceptual revolution, a paradigm shift that has far reaching consequences for the very definition of physics" [9].

For almost half a century physicists have been trying to understand the fundamental principles governing the flow of vehicular traffic using theoretical approaches based on statistical physics [3,4,10-13]. The approach of a physicist is usually quite different from that of a traffic engineer. A physicist would like to develop a model of traffic by incorporating only the most essential ingredients which are absolutely necessary to describe the general features of typical real traffic. The theoretical analysis and computer simulation of these models not only provide deep insight into the properties of the model but also help in better understanding of the complex phenomena observed in real traffic. Our aim in this review is to present a critical survey of the progress made so far towards understanding the fundamental aspects of traffic phenomena from the perspective of statistical physics.

There are two different conceptual frameworks for modeling traffic. In the "coarse-grained" fluid-dynamical description, the traffic is viewed as a compressible fluid formed by the vehicles but these individual vehicles do not appear explicitly in the theory. In contrast, in the so-called "microscopic" models of vehicular traffic attention is explicitly focused on individual vehicles each of which is represented by a "particle"; the nature of the interactions among these particles is determined by the way the vehicles influence each others' movement. In other words, in the "microscopic" theories vehicular traffic is treated as a system of interacting "particles" driven far from equilibrium. Thus, vehicular traffic offers the possibility to study various fundamental aspects of the dynamics of truly nonequilibrium systems which are of current 
interest in statistical physics [14-17].

In order to provide a broad perspective, we describe both "macroscopic" and "microscopic" approaches although we put more emphasis on the latter. Sometimes the phenomenological equations of traffic flow in the "macroscopic" models can be obtained from microscopic considerations in the same spirit in which macroscopic or phenomenological theories of matter are derived from their molecular-theoretic description.

At present, even within the conceptual framework of "microscopic" approach, there are several different types of mathematical formulations of the dynamical evolution of the system. For example, the probabilistic description of vehicular traffic in the kinetic theory is developed by appropriately modifying the kinetic theory of gases. On the other hand, a deterministic description of the motion of individual vehicles is provided by the so-called car-following theories which are based on the basic principles of classical Newtonian dynamics. In contrast, the so-called particle-hopping models describe traffic in terms of a stochastic dynamics of individual vehicles. We explain the guiding principles behind all these formulations. But we discuss in detail the results obtained mainly from the investigations of the recently developed "particle-hopping" models which are usually formulated using the language of cellular automata (CA) [18]. At present, there is no traffic model yet which can account for all aspects of vehicular traffic. In this review we consider a wide variety of CA models which describe various different types of traffic phenomena.

We map the particle-hopping models of vehicular traffic onto some other model systems; these mappings indicate the possibility of exploiting powerful techniques, used earlier for other systems, to study traffic models and, sometimes, enable us to obtain results for traffic models directly from the known results for models of other systems. We present pedagogical summaries of the statistical mechanical treatments of the CA models of traffic. We critically examine the regimes of validity of the approximation schemes of analytical calculations which we illustrate with explicit calculations in those limiting cases where these usually yield exact results. The results of the theoretical analysis of these models are compared with those obtained from computer simulations and, wherever possible, with the corresponding empirical results from real traffic. We also compare vehicular traffic with many other similar physical systems to show the ubiquity of some physical phenomena.

Computer simulations are known to provide sufficiently accurate quantitative results when analytical treatments require approximations which are too crude to yield results of comparable accuracy. In this review we demonstrate how computer simulations often help in getting deep insight into various phenomena involved in traffic and in qualitative understanding of the basic principles governing them thereby avoiding potentially hazardous experiments with real 
traffic. Computer simulations of the "microscopic" models of traffic have not only attracted the attention of a growing number of statistical physicists in the recent years, but have also been received positively by many traffic engineers. The ongoing research efforts to utilize computer simulations of the microscopic models for practical applications in planning and design of transportation networks have been reviewed very recently by Nagel et al. [19].

Our review is complimentary to those published in the recent years by Helbing [13] and by Nagel et al. [19]. A large number of important papers on traffic published in recent years are based on the particle-hopping models. But these works have received very little attention in [13]. We discuss the methods and results for the particle-hopping models in great detail in this review after explaining the basic principles of all the theoretical approaches. Moreover, we focus almost exclusively on the fundamental principles from the point of view of statistical physics while Nagel et al. [19] emphasize practical applications which are directly relevant for traffic engineering.

At this point a skeptic may raise a serious question: "can we ever predict traffic phenomena with statistical mechanical theories without taking into account effects arising from widely different human temperaments and driving habits of the individual drivers?" We admit that, unlike the particles in a gas, a driver is an intelligent agent who can "think", make individual decisions and "learn" from experience. Besides, the action of the driver may also depend on his/her physical and mental states (e.g., sorrow, happiness, etc.). It is also true that the behavior of each individual driver does not enter explicitly into the "microscopic" models of traffic. Nevertheless, as we shall show in this review, many general features of traffic can be explained in general terms with these models provided the different possible behavioural effects are captured collectively through a probabilistic description which requires only a few phenomenological parameters. Similar strategies have been suggested also for capturing the behavioural effects of some individual traders in financial markets collectively through probabilistic descriptions [1]. These probabilistic descriptions make the dynamics of the models intrinsically stochastic.

Throughout this article the terms vehicle (or, car) and driver are used interchangeably, although each of these terms usually refers to the composite unit consisting of the vehicle and the driver. The main questions addressed by physicists are posed as problems in Section 2. As a motivation we present some relevant empirically observed general features of real traffic as well as their plausible phenomenological explanations in Section 3. The following sections review the different theoretical approaches. The classification of the models into different classes is not unique. Mostly we choose a classification according to the use of discrete or continuous space, time and state variables. The conceptual basis of the older theoretical approaches, namely, the fluid-dynamical theories, the kinetic theories and the car-following theories, are explained, in 
the Sections 4,5 and 6, respectively, where the corresponding recent developments are also summarized. These model classes are continuous in space, time and state variables. Some coupled map lattice models of traffic are considered in Section 7. They are discrete in time. CA models are discrete in space, time and state variables. The model suggested by Nagel and Schreckenberg (NaSch) [20] is the minimal model of traffic on highways; the theoretical results on various aspects of this model are discussed in Section 8 where the nature of the spatio-temporal organization of vehicles are also investigated and the fundamental question of the (im-)possibility of any dynamical phase transition in the NaSch model is addressed. Various generalizations and extensions of the NaSch model (including those for multi-lane traffic) are reviewed in Section 9. The occurrence of self-organized criticality in the so-called cruisecontrol limit of the NaSch model is pointed out. It is demonstrated how additional "slow-to-start" rules of CA can give rise to metastability, hysteresis and phase-separation in the generalized NaSch models, in qualitative agreement with empirical observations. In Section 10 the formation and "coarsening" of platoons of vehicles are investigated in an appropriate generalization of the NaSch model with one type of quenched randomness; your attention is drawn to the formal analogy between this phenomenon and the Bose-Einstein condensation. In this section the effects of other kinds of quenched disorder on the nature of the steady-states of the NaSch model are also considered. In Section 11 we present some other CA models of highway traffic which are not directly related to the NaSch model. The Biham-Middleton-Levine (BML) [21] model is the earliest CA model of traffic in idealized networks of streets in cities; it exhibits a first order phase transition. A critical review of this model is presented in Section 12 together with a list of its generalizations which have been reported so far. Furthermore a marriage of the NaSch description of traffic and the BML model, which has led to the development of a novel model of city traffic, is explained. A brief status report of the ongoing efforts to make practical use of the theoretical models for traffic engineering is also presented. The similarities between various particle-hopping models of traffic and some other systems far from equilibrium are pointed out in Section 13 followed by the concluding Section 14 where your attention is also drawn towards challenging open questions. Several Appendices deal mostly with more technical aspects

of some important calculations, but are not necessary for an understanding of the main text.

\section{Fundamental and practical questions}

The aim of basic research in traffic science is to discover the fundamental laws governing traffic systems. The main aim of traffic engineering is on planning, design and implementation of transportation network and traffic control 
systems. Statistical physicists have been contributing to traffic science by developing models of traffic and drawing general conclusions about the basic principles governing traffic phenomena by studying these models using the tools of statistical physics. Moreover, using these models, statistical physicists have also been calculating several quantities which may find practical applications in traffic engineering. Furthermore, several groups of statistical physicists are currently also engaged in developing strategies for fast on-line simulation and traffic control so as to optimize traffic flow; significant contributions to this traditional domain of traffic engineering can reduce the financial burden on the governments.

\subsection{Some fundamental questions}

Because of the apparent similarities between the "microscopic" models of traffic and macroscopic samples of ionic conductors in the presence of external electric field, the tools of statistical mechanics seem to be the natural choice for studying these models. However, the actual calculation of even the steadystate properties of traffic from the "microscopic" models is a highly difficult problem because (apart from the human element involved) (a) the vehicles interact with each other and (b) the system is driven far from equilibrium, although it may attain a nonequilibrium steady-state.

In principle, the time-independent observable properties of large pieces of matter can be calculated within the general framework of equilibrium statistical mechanics, pioneered by Maxwell, Boltzmann and Gibbs, provided the system is in thermal equilibrium. Of course, in practice, it may not be possible to carry out the calculations without making approximations because of the interactions among the constituents of the system. Some time-dependent phenomena, e.g., fluctuation and relaxation, can also be investigated using the Linear Response Theory provided the system is not too far from equilibrium. Unfortunately, so far there is no general theoretical formalism for dealing with systems far from equilibrium. Moreover, the condition of detailed balance does not hold [22] although a condition of pairwise balance [23] holds for some special systems driven far from equilibrium.

The dynamical phases of systems driven far from equilibrium are counterparts of the stable phases of systems in equilibrium. Some of the fundamental questions related to the nature of these phases are as follows.

(i) What are the various dynamical phases of traffic? Does traffic exhibit phase-coexistence, phase transition, criticality [24,25] or self-organized criticality $[26,27]$ and, if so, under which circumstances?

(ii) What is the nature of fluctuations around the steady-states of traffic? Analogous phenomenon of the fluctuations around stable states in equilibrium is 
by now quite well understood.

(iii) If the initial state is far from a stationary state of the driven system, how does it evolve with time to reach a truly steady-state? Analogous phenomena of equilibration of systems evolving from metastable or unstable initial states through nucleation (for example, in a supersaturated vapour) or spinodal decomposition (for example, in a binary alloy) have also been studied earlier extensively [28].

(iv) What are the effects of quenched (static or time-independent) disorder on the answers of the questions posed in (i)-(iii) above?

\subsection{Some practical questions}

Let is first define some characteristic quantitative features of vehicular traffic. The flux $J$, which is sometimes also called flow or current, is defined as the number of vehicles crossing a detector site per unit time [29]. The distance from a selected point on the leading vehicle to the same point on the following vehicle is defined as the distance-headway [29]. The time-headway is defined as the time interval between the departures (or arrivals) of two successive vehicles recorded by a detector placed at a fixed position on the highway [29]. The distributions of distance-headways and time-headways are regarded as important characteristic of traffic flow. For example, larger headways provide greater margins of safety whereas higher capacities of the highway require smaller headways.

Let us now pose some questions which are of practical interest in traffic engineering.

(a) What is the relation between density $c$ and flux $J$ ? In traffic engineering, this relation is usually referred to as the fundamental diagram.

(b) What are the distributions of the distance-headway and time-headway?

(c) How should on- and off-ramps be designed?

(d) Does an additional lane really lead to an improvement?

(e) What are the effects of a new road on the performance of the road network?

(f) What type of signaling strategy should be adopted to optimize the traffic flow on a given network of streets and highways?

(g) The generalized travelling salesman problem: Is the shortest trip also the fastest? 


\section{Some empirical facts and phenomenological explanations}

For several reasons, it is difficult to obtain very reliable (and reproducible) detailed empirical data on real traffic. First of all, unlike controlled experiments performed in the conventional fields of research in physical sciences, it is not possible to perform such laboratory experiments on vehicular traffic. In other words, empirical data are to be collected through passive observations rather than active experiments. Secondly, unambiguous interpretation of the collected data is also often a subtle exercise because traffic states depend on several external influences, e.g. the weather conditions. The systematic investigation of traffic flow has a quite long history [30-32]. Although we now have a clear understanding of many aspects of real traffic several other controversial aspects still remain intellectual challenges for traffic scientists. In this section we give an overview of some of the well understood experimental findings, which are relevant for our theoretical analysis in the following sections. Moreover, wherever possible, we provide phenomenological explanations of these empirically observed traffic phenomena. Furthermore, we shall also mention some of the more recent empirical observations for which, at present, there are no generally accepted explanations.

\subsection{Acceleration noise}

In general, because of the different human temperaments and driving habits, different drivers react slightly differently to the same conditions on a highway, even when no other vehicle influences its motion. Consequently, even on an empty stretch of a highway, a driver can neither maintain a constant desired speed nor accelerate in a smooth fashion. In addition to the type of the highway (i.e., the surface conditions, frequency of the curves, etc.) the driver-to-driver fluctuation of the acceleration also depends on the density of vehicles on the highway. The root-mean-square deviation of the acceleration of the vehicles is a measure of the so-called acceleration noise. The distributions of the accelerations have been measured since mid nineteen fifties and are well documented [33,34].

\subsection{Formation and characterization of traffic jams}

Traffic jam is the most extensively studied traffic phenomenon. Traffic jams can emerge because of various different reasons. Most often traffic jams are observed at bottlenecks, e.g. lane-reductions or crossings of highways [35]. At bottlenecks the capacity of the road is locally reduced thereby leading to the formation of jams upstream traffic. Downstream the bottleneck, typically, a 
free-flow region is observed. In addition, traffic accidents, which also lead to a local reduction of the capacity of the highway, can give rise to traffic jams.


Fig. 1. Experimental flow speed-diagrams near on- and off ramps of a three lane highway. The upper part of the figure shows the empirical results. Each dot represents a five-minute average of the local measurement. The lines serve merely as guide to the eyes. The lower part of the figure shows the location of the detectors (from [32]).

Fig. 1 shows as an example empirical data of the velocity at a three-lane highway close to an on- and off-ramp [32]. The data show that downstream the bottleneck (at detector $\mathrm{C}$ ) no slow vehicle has been recorded. In contrast, in the merging regime near detector A vehicles often have to move slowly. In between the on- and off-ramps the vehicles move with larger velocities compared to those in location A although the number of vehicles passing detector $\mathrm{B}$ is maximal. Therefore, the on-ramp causes a local reduction of the capacity of the highway.

Perhaps, what makes the study of traffic jams so interesting is that jams often appear, as if, from nowhere (apparently without obvious reasons) suddenly on crowded highways; these so-called "phantom jams" " are formed by spontaneous fluctuations in an otherwise streamlined flow. Direct empirical evidence for this spontaneous formation of jams was presented by Treiterer [36] by analyzing a series of aerial photographs of a multi-lane highway. In Fig. 2 the picture from [36] is redrawn. Each line represents the trajectory of an individual vehicle on one lane of the highway'. The space-time plot (i.e., the trajectories $x(t)$ of the vehicles) shows the formation and propagation of a traffic jam. In the beginning of the analysed time vehicles are well separated from each other. Then, due to fluctuations, a dense region appears which, finally, leads to the formation of a jam. The jam remains stable for a certain

\footnotetext{
5 "Stau aus dem Nichts" in german

6 The discontinuous trajectories correspond to vehicles changing the lane.
} 
period of time but, then, disappears again without any obvious reason. This figure clearly establishes not only the spontaneous formation of traffic jam but also shows that such jams can propagate upstream (opposite to the direction of flow of the vehicles). Moreover, it is possible that two or more jams coexist on a highway.



Fig. 2. Trajectories of individual vehicles on a single lane of a multi-lane highway. The trajectories were drawn from aerial photographs. During the analyzed time-interval the spontaneous formation, propagation and dissolution of a jam has been observed (from [36]).

A more detailed analysis of traffic jams in absence of hindrances has been given by Kerner and Rehborn [37-39] who pointed out the following characteristic features of wide jams. They found that the upstream velocity and, therefore, the outflow from a jam is approximately constant. The outflow from a jam and the velocity of the jam fronts are now regarded as two important empirical parameters of highway traffic which can be used for calibrating theoretical models.

\subsection{Flux-density relation}

Obviously, traffic flow phenomena strongly depend on the occupancy of the road. What type of variation of flux and average velocity $\langle v\rangle$ with density $c$ can 
one expect on the basis of intuitive arguments? So long as $c$ is sufficiently small, the average speed $\langle v\rangle$ is practically independent of $c$ as the vehicles are too far apart to interact mutually. Therefore, at sufficiently low density of vehicles, practically "free flow" takes place. However, from the practical experience that vehicles have to move slower with increasing density, one expects that at intermediate densities,

$$
\frac{d\langle v\rangle}{d c} \leq 0
$$

when the forward movement of the vehicles is strongly hindred by others because of the reduction in the average separation between them. A fasterthan-linear monotonic decrease of $\langle v\rangle$ with increasing $c$ can lead to a maximum [29] in the flux $\langle J\rangle=\langle c v\rangle$ at $c=c_{m}$; for $c<c_{m}$, increasing $c$ would lead to increasing $\langle J\rangle$ whereas for $c>c_{m}$ sharp decrease of $\langle v\rangle$ with increase of $c$ would lead to the overall decrease of $\langle J\rangle$. However, contrary to this naive expectation, in recent years some nontrivial variation of flux with density have been observed. The nature of the variation of the flux with the density is still not clearly understood [40] since the details of the complex experimental setup can strongly influence the empirical results.



Fig. 3. Empirical data for flow and occupancy. The data have been collected by counting loops on a Canadian highway. Both the occupancy and the flow have been directly measured by the detector. Each point in the diagram corresponds to an average over a time interval of five minutes (from [40]).

Fig. 3 shows typical time averaged local measurements of the density and flow 
which have been obtained from the Queen Elizabeth Way in Ontario (Canada) [40]. At low densities the data indicate a linear dependence of the flow on the density. In contrast strong fluctuations of the flow at large densities exist which prevents a direct evaluation of the functional form at high densities.



Fig. 4. Schematic representation of fundamental diagrams consistent with empirical investigations (from [40]).

In order to use the empirical results for a theoretical analysis it is often more convenient to use the mean-values of the flow at a given density. Fig. 4 shows a collection of possible forms of averaged fundamental diagrams consistent with empirical data [40]. While the discontinuity of the fundamental diagram now seems to be well established [41] no clear answer can be given to the question on the form of the diagram in the free-flow or high-density regime. In the low density regime linear as well as non-linear functional forms of the fundamental diagrams have been suggested. For the high density branch no consistent picture for the high density branch exists. Here the results strongly depend on the specific road network.

In several situations it has been observed that $J$ does not depend uniquely on $c$ in an intermediate regime of density; it indicates the existence of hysteresis effects and meta-stable states. In the context of traffic flow, hysteresis effects have the following meaning: if a measurement starts in the free-flow 




Fig. 5. Time-traced measurements of the flow (from [40]). Each data point corresponds to a time-averaged value of the flow and density obtained from a local measurement. The average was performed over five minute intervals.

regime, an increase of the density leads to an increase of the flow. However, beyond a certain density, a further increase of the density leads to a discontinuous reduction of the stationary flow ("capacity drop") and jams emerge. The corresponding fundamental diagram (Fig. 4 A, B) then has the so-called 'inverse- $\lambda$ form'. Figure 5 shows an experimental verification of a hysteresis loop [40] at a transition from a free flow to a congested state.

Recent empirical observations which have been obtained near a crossing of highways exhibit [41] a flat plateau (i.e., a density-indepedent flux) over an intermediate regime of density of the vehicles.

\subsection{Microscopic states of traffic flow and phase transitions}

The results for the flux-density relation already suggest the existence of at least two different dynamical phases of vehicular traffic on highways, namely a freeflow phase and a congested phase. In the free-flow regime all vehicles can move with high speed close to the speed limit. The nature of the congested traffic is still under debate. Careful empirical observations in the recent years indicate the existence of two different congested phases, namely, the synchronized phase and the stop-and-go traffic phase [38,39,42-44]. Vehicles move rather slowly 
in the synchronized states, as compared to the free-flow states, but the flux in the synchronized states can take a values close to the optimum value because of relatively small headways. Besides, non-trivial strong correlations between the density on different lanes exist in the synchronized state $[38,44,45]$ which actually motivates the notation synchronized traffic. The stop-and-go traffic differs from the synchronized states in the sense that every vehicle inside the jams come to a complete halt for a certain period of time.


Fig. 6. Fundamental diagrams corresponding to different types of synchronized flow. Measurements which correspond to synchronized traffic of type (i) are localised in a narrow region of the flow density plane, while synchronized states of type (ii) are similar to the results of free-flow states. In contrast for synchronized traffic of type (iii) the time-traced measurements show an unsystematic behaviour.

Following Kerner three different types of synchronized traffic can be distringuished by the time-dependent behaviour of the density and flow $[38,39,44]$. In synchronized traffic of type (i) constant values of density and flow can be observed during a long period of time. In synchronized traffic of type (ii) patterns of density and flow quite similar to free flow states have been observed. The differences between synchronized states of type (ii) and free-flow are given by the reduced average velocities and the alignment of the speeds on different lanes in synchronized traffic. Moreover irregular patterns of time-traced measurements of the flow have been found in synchronized traffic (see Fig. 6) of type (iii).

\subsection{Time- and distance-headways}

The flux $J$ can be written as $J=N / T$ where $T=\sum_{i=1}^{N} t_{i}$ is the sum of the time-headways recorded for all the $N$ vehicles. Hence, $J=1 / T_{a v}$ where $T_{a v}=(1 / N) \sum_{i} t_{i}$ is the average $\mathrm{TH}$. Therefore, the $\mathrm{TH}$ distribution contains more detailed informations on traffic flow than that available from the flux alone. With the variation of density $c$ of the vehicles, $T_{a v}$ exhibits a minimum at $c=c_{m}$ where the flux is maximum [29]. 




Fig. 7. Time-headway distribution for different density regimes. Top: In free-flow traffic the $\Delta t$-distribution is dominated by two peaks at $0.8 \mathrm{sec}$ and $1.8 \mathrm{sec}$. Middle: In synchronized traffic cars with narrow time gaps are to find as well as a dominating peak. Bottom: In stop-and-go traffic short time headways are surpressed. The peak at $1.8 \mathrm{sec}$ remains since vehicles are leaving the jam with a typical temporal headway of approximately $2 \mathrm{sec}$.

The results discussed in the preceeding subsections are based on time averaged local measurements. But it is also very useful to analyze the single-vehicle data directly $[46-48,41]$. The single-vehicle data allows calculation of the timeheadway distributions [41]. All the time-headway distributions in the free-flow regime show a two peak structure. The first peak at $\Delta t=0.8 \mathrm{sec}$ represents the global maximum of the distribution. On a microscopic level these short timeheadways correspond to platoons of some vehicles traveling very fast - their drivers are taking the risk of driving "bumper-to-bumper" with a rather high speed. These platoons are the reason for the occurrence of high-flow states in free traffic. The corresponding states exhibit metastability, i.e. a perturbation of finite magnitude and duration is able to destroy such a high-flow state [39]. Additionally, a second peak emerges at $\Delta t=1.8 \mathrm{sec}$ which can be associated with a typical drivers' urge to maintain a temporal headway of $\approx 2 \mathrm{sec}$ (which is the safe distance recommended in driving schools).

Surprisingly, the small time headways have much less weight in congested traffic. Only the peak at $\Delta t=1.8 \mathrm{sec}$ is recovered, where the time headway distribution now takes the maximum value. But nevertheless, the small time headways $(\Delta t<1.8 \mathrm{sec})$ contribute significantly in synchronized traffic. In stop-and-go traffic only the 1.8 sec-peak remains and short time-headways are surpressed. The asymptotic behavior is rather unsystematic and reflects the dynamics of vehicles inside the jams. 




Fig. 8. Speed-distance relation in free and congested traffic. It turns out that the typical velocities at large distance headways strongly depend on the present traffic state (from [41]).

Another important result characterizing the microscopic states is the dependence of the velocity of individual vehicles on the distance headway (see Fig. 8). This function is also of great importance for theoretical approaches, e.g. it is used as input for the so-called optimal velocity model. In the free-flow regime it is evident that the asymptotic velocity is reached already for small distanceheadways. The slope of the velocity function is much lower than that in the free-flow regime. Surprisingly, the asymptotic velocity depends strongly on the microscopic state as well as on the density, e.g., in dense traffic low velocities of the vehicles are also observed even when large distance headways are available.

\section{Fluid-dynamical Theories of vehicular traffic}

When viewed from a long distance, say, an aircraft, flow of fairly heavy traffic appears like a stream of a fluid. Therefore, a "macroscopic" theory of traffic can be developed, in analogy with the hydrodynamic theory of fluids, by treating traffic as an effectively one-dimensional compressible fluid (a continuum) [49]. We follow the convention that traffic is flowing from left to right.

Suppose, $c(x ; t)$ and $J(x ; t)$ are the "coarse-grained" density and flux at an arbitrary location $x$ at an arbitrary instant of time $t$. The equation of continuity 
for the fluid representing traffic is

$$
\frac{\partial c(x ; t)}{\partial t}+\frac{\partial J(x ; t)}{\partial x}=\sum_{i=1}^{J_{\text {in }}} \alpha_{i}\left(x-x_{i} ; t\right)-\sum_{j=1}^{J_{\text {out }}} \beta_{j}\left(x-x_{j} ; t\right)
$$

where the first and the second terms on the right hand side take care of the sources and sinks, respectively, at the $J_{i n}$ on-ramps situated at $x_{i}(i=$ $\left.1,2, \ldots, J_{\text {in }}\right)$ and $J_{\text {out }}$ off-ramps situated at $x_{j}\left(j=1,2, \ldots, J_{\text {out }}\right)$. We can write $\alpha_{i}\left(x-x_{i} ; t\right)$ and $\beta_{j}\left(x-x_{j} ; t\right)$ as

$$
\alpha_{i}\left(x-x_{i} ; t\right)=\alpha_{i}^{0}(t) \phi_{i}\left(x-x_{i}\right) \quad \text { and } \quad \beta_{j}\left(x-x_{j} ; t\right)=\beta_{j}^{0}(t) \phi_{j}\left(x-x_{j}\right)
$$

where $\phi_{i}\left(x-x_{i}\right)$ and $\phi_{j}\left(x-x_{j}\right)$ describe the spatial distribution of the incoming and outgoing flux, respectively, while $\alpha_{i}^{0}(t)$ and $\beta_{j}^{0}(t)$ account for the corresponding temporal variations.

In the following subsections, for simplicity, we shall consider a given stretch of highway with no entries or exits. In such special situations the equation of continuity reduces to the simpler form [50]

$$
\frac{\partial c(x ; t)}{\partial t}+\frac{\partial J(x ; t)}{\partial x}=0
$$

One cannot get two unknowns, namely, $c(x ; t)$ and $J(x ; t)$ (or, equivalently, $v(x ; t))$ by solving only one equation, namely $(4)$, unless they are related to each other. In order to proceed further, one needs another independent equation, say, for $v(x ; t)$; we shall write down such an equation later in subsection 4.4. An alternative possibility, which Lighthill and Whitham [50] adopted in their pioneering work, is to assume that $J(x ; t)$ is determined primarily by the local density $c(x ; t)$ so that $J(x ; t)$ can be treated as a function of only $c(x ; t)$. Consequently, the number of unknown variables is reduced to one as, according to this assumption, the two unknowns $c(x ; t)$ and $J(x ; t)$ are not independent of each other.

\subsection{Lighthill-Whitham theory and kinematic waves}

As a first approximation, let us begin with Lighthill-Whitham assumption that

$$
J(x ; t)=j(c(x ; t))
$$

where $j(c)$ is a function of $c$. The functional relation (5) between density and flux cannot be calculated within the framework of the fluid-dynamical theory; this must be either taken as a phenomenological relation extracted from 
empirical data or derived from more microscopic considerations. In general, the flux-density curve implied by equation (5) need not be identical with the fundamental diagram in the steady-state.

Under the assumption (5), the $x$-dependence of the local flux $J(x ; t)$ arises only from the $x$-dependence of $c(x ; t)$. Alternatively, since $J(x ; t)=c(x ; t) v(x ; t)$, assuming $v(x ; t)$ to depend only on $c(x ; t)$ the $x$-dependence of $v(x ; t)$ arises only from the $x$-dependence of $c(x ; t)$. Using (5) the equation of continuity (4) can be expressed as

$$
\frac{\partial c(x ; t)}{\partial t}+\frac{\partial c(x ; t)}{\partial x}\left[v(x ; t)+c(x ; t) \frac{d v}{d c}\right]=\frac{\partial c(x ; t)}{\partial t}+v_{g} \frac{\partial c(x ; t)}{\partial x}=0
$$

where $v_{g}=d J / d c$. Note that the equations (5) and (6) form the complete system of dynamical equations governing traffic flow in this first approximation. However, the equation (6) is non-linear because, in general, $v_{g}=d J / d c=$ $v(c)+c \frac{d v(c)}{d c}$ depends on $c$. If $v_{g}$ were a constant $v_{0}$, independent of $c$, equation (6) would become linear and the general solution would be of the form $c(x ; t)=f\left(x-v_{0} t\right)$ where $f$ is an arbitrary function of its argument. In that case, the solution of any particular problem would be found by merely matching the function $f$ to the corresponding given initial and boundary conditions. Such a solution describes a density wave motion as an initial density profile would get translated by a distance $v_{0} t$ in a time interval $t$ without any change in its shape. However, the non-linearity of the equation (6) gives rise to subtleties which are essential to capture at least some aspects of real traffic.

The solution of the nonlinear equation (6) is of the general form

$$
c(x ; t)=F\left(x-v_{g} t\right)
$$

where $F$ is an arbitrary function of its arguments. If we define a wave to be "recognizable signal that is transferred from one part of a medium to another with a recognizable velocity of propagation" [51] then the solutions of the form (7) can be regarded as a "density wave". There are several similarities between the density wave and the more commonly encountered waves like, for example, acoustic or elastic waves, But, the acoustic or elastic waves are solutions of linearized partial differential equations whereas the equation (6) is nonlinear and, hence, $v_{g}$ is $c$-dependent. Besides, the waves of the type (7) are called kinematic waves [50-52] to emphasize their purely kinematic origin, in contrast to the dynamic origin of the accoustic and elastic waves. From the initial given density profile $c(x ; 0)$ the profile $c(x ; \Delta t)$ at time $\Delta t$ can be obtained by moving each point on the initial profile a distance $v_{g}(c) t$ to the right; obviously, the distance moved is different for different values of $c$. The time-evolution of the density profile can be shown graphically $[50,51]$ on the space-time diagram (i.e., the $x-t$ plane) where an arbitrary point $x_{0}$ on the $t=0$ axis moves along a straight line of slope $v_{g}(c)$ if the initial density at $x_{0}$ is 
c. These straight lines are referred to as characteristics; different characteristics corresponding to different $c$ have different slopes $v_{g}(c)$.

The speed $v_{g}(c)$ of the density wave should not be confused with $v(c)$, the actual speed of the continuum fluid representing traffic. In fact, at any instant of time $v(x ; t)$ can be obtained from the corresponding density profile $c(x ; t)$ by using the relation $v(x ; t)=j(c(x ; t)) / c(x ; t)$. Moreover, since $v_{g}=v(c)+c \frac{d v(c)}{d c}$ and since $\frac{d v(c)}{d c}<0$, the speed of the density wave is less than that of the fluid. Therefore, the density wave propagates backward relative to the traffic and the drivers are thereby warned of density fluctuations ahead downstream. Furthermore, the density wave moves forward or backward relative to the road, depending on whether $c<c_{m}$ or $c>c_{m}$ where $c_{m}$ corresponds to the maximum in the function $j(c)$.

When $J(c)$ is convex, i.e., $d^{2} J / d c^{2}<0$, we have $d v_{g} / d c<0$; consequently, higher values of $c$ propagate slower than lower values of $c$ thereby distorting the initial density profile. On the other hand, when $d v_{g} / d c>0$ higher values of $c$ propagate faster and the distortion has the opposite tendency as compared to the case of $d v_{g} / d c<0$. In both the situations the distortion of the initial density profile is caused by the $c$-dependence of $v_{g}$ which arises from the nonlinearity of the equation (6). The distortion of the density profile with time can also be followed on the space-time diagram. If $d v_{g} / d c<0$, in regions of decreasing density (i.e., $c\left(x_{1}\right)>c\left(x_{2}\right)$ for $x_{1}<x_{2}$ ) the characteristics move away from each other whereas, in regions of increasing density, the characteristics move towards each other.

When two characteristic lines on the space-time diagram intersect the density would be double-valued at the point of intersection. We can avoid this apparently impossible scenario by the following interpretation: When two characteristic lines intersect a shock wave is generated. By definition, a shock represents a mathematical discontinuity in $c$ and, hence, also in $v$. The speed of a shock wave is given by

$$
v_{s}=\frac{J\left(c^{+}\right)-J\left(c^{-}\right)}{c^{+}-c^{-}}
$$

where $c^{+}$and $c^{-}$are, respectively, the densities immediately in front (downstream) and behind (upstream) the shock while $J\left(c^{+}\right)$and $J\left(c^{-}\right)$represent the corresponding downstream and upstream fluxes, respectively. Note that the shock wave moves downstream (upstream) if $v_{s}$ is positive (negative). Often the shock is weak in the sense that the relative discontinuity $\left(c^{+}-c^{-}\right) / c^{-}$is small and in such cases the shock wave speed tends to $v_{g}=d J / d c$. As a shock separates a section of high an low densities of the model, it corresponds to a section of a highway where a free-flow and a congested regime is present. In particular for large differences between $c^{+}$and $c^{-}$the velocity of the shock can be interpreted as the velocity of a backwards moving jam. 
One advantage of the kinematic approach outlined above over any dynamic approach is that the dynamical equation, which will be given in Sec. 4.4, is difficult to derive from basic first principles and usually involve quite a few phenomenological parameters and even a phenomenological function. On the other hand, the only input needed for the kinematic approach is the phenomenological function $J(c)$ which can be obtained from empirical data.

\subsection{Diffusion term in Lighthill-Whitham theory and its effects}

Let us now make improvement over the original Lighthill-Whitham theory, which is based on the first approximation (5). We now assume that the local flux $J(x ; t)$ is determined not only by the local density $c(x ; t)$ but also by the gradient of the density. In other words, we replace the assumption (5) by

$$
J(c)=j(c)-D \frac{\partial c}{\partial x}
$$

where $D$ is a positive constant. Note that, for fixed $c(x ; t)$ (and, hence, fixed $j(c)$ ), a positive (negative) density gradient leads to a lower (higher) flux as the drivers are expected to reduce (increase) the speed of their vehicles depending on whether approaching a more (less) congested region. Using the relation (9) in the equation of continuity (4) we now get

$$
\frac{\partial c(x ; t)}{\partial t}+v_{g} \frac{\partial c(x ; t)}{\partial x}=D \frac{\partial^{2} c(x ; t)}{\partial x^{2}}
$$

where $v_{g}(c)=\frac{d j(c)}{d c}$. The equation (10) reduces to the equation (6) when $D=0$. The nonlinearity and diffusion have opposite effects: the term $v_{g}(c) \frac{\partial c}{\partial x}$ leads to "steepening" and ultimate "breaking" of the wave whereas the term $D \partial^{2} c / \partial x^{2}$ smoothens out the profile. Nonvanishing $D$ also leads to a non-zero width of the shock wave.

\subsection{Greenshields model and Burgers equation}

So far in the preceding subsections we have not considered any specific form of the function $j(c)$ relating flux with density. One can start with the simplest (differentiable) approximation capturing the basic form of the fundamental diagram,

$$
J=v_{\max } c(1-c) .
$$

Note that $v_{\max }$ in (11) is a phenomenological parameter and it is interpreted to be the maximum average speed for $c \rightarrow 0$. In traffic science and engineering, 
one usually uses $1-c / c_{\text {jam }}$ instead of $1-c$ in the equation (11) and the corresponding form of the relation between $J$ and $c$ is known as the Greenshields [30] model. Substituting (11) into the equation (10) we get

$$
\frac{\partial c(x ; t)}{\partial t}+v_{\max } \frac{\partial c(x ; t)}{\partial x}-2 v_{\max } c \frac{\partial c(x ; t)}{\partial x}=D \frac{\partial^{2} c}{\partial x^{2}}
$$

Introducing the linear transformation of variables

$$
x=v_{\text {max }} t^{\prime}-x^{\prime} ; \quad t=t^{\prime}
$$

one gets

$$
\frac{\partial c(x ; t)}{\partial t^{\prime}}+2 v_{\max } c \frac{\partial c(x ; t)}{\partial x^{\prime}}=D \frac{\partial^{2} c}{\partial x^{\prime 2}}
$$

which is the (deterministic) Burgers equation [51,53]. Note that the transformation (13) takes one from the space-fixed coordinate system $(x, t)$ to a coordinate system $\left(x^{\prime}, t^{\prime}\right)$ that moves with uniform speed $v_{\max }$; so, vehicles moving with speed $v_{\max }$ with respect to the coordinate system $(x, t)$ do not move at all with respect to the coordinate system $\left(x^{\prime}, t^{\prime}\right)$.

The advantage of this route to the theory of traffic flow is that the Burgers equation (14) can be transformed further into a diffusion equation, thereby getting rid of the nonlinearity, through a nonlinear transformation called the Cole-Hopf transformation [51]. Since it is straightforward to write down the formal solution to the diffusion equation, one can see clearly the role of the coefficient $D$ and the nature of the solutions in the limit $D \rightarrow 0$.

If equation (6) is assumed to be the only equation governing traffic flow then an inhomogeneous initial state can lead to a shock wave but the amplitude of the shock wave decreases with time and eventually the shock wave fades out leading to a homogeneous steady state in the limit $t \rightarrow \infty$. Leibig [54] has studied how a random initial distribution of steps in the density profile evolves with time in this theory. No traffic jam forms spontaneously from a state of uniform density at this level of sophistication of the fluid-dynamical approach.

\subsection{Navier-Stokes-like momentum equation and consequences}

Corresponding to the assumption (9) we can write a velocity equation

$$
v(x ; t)=v(c(x ; t))-\frac{D}{c} \frac{\partial c(x ; t)}{\partial x}
$$


where $v(c)=\frac{j(c(x ; t))}{c(x ; t)}$. In the kinematic approach discussed so far in the preceding subsections it is implicitly assumed that, following any change in the local density (and density gradient) is followed by an immediate response (without delay) of the velocity field. For a more realistic description, the local speed should be allowed to relax after a non-zero delay time $t_{d}$. So, it seems natural to treat the right hand side in (15) as a desired local velocity at $x$ and write the total derivative $d v / d t$ of $v$ with respect to time as [55-58]

$$
c \frac{\partial v}{\partial t}+v \frac{\partial v}{\partial x}=\frac{c}{t_{d}}\left[v_{s a f e}(c)-v\right]-D \frac{\partial c(x ; t)}{\partial x}
$$

where the function $v_{\text {safe }}(c)$ is identical to $v(c)$. Note that $v_{\text {safe }}(c)$ is a monotonically decreasing function of $c$, i.e, $d v_{\text {safe }} / d c<0$. Equation (16) is an additional dynamical equation describing the time-dependence of the velocity $v(x ; t)$.

Now let us interpret the two terms on the right hand side of (16). The phenomenological function $v_{\text {safe }}(c)$ gives the safe speed, corresponding to the vehicle density $c$, that is achieved in time-independent and homogeneous traffic flow and $t_{d}$ is the corresponding average relaxation time. Next, note that the term $D \frac{\partial c(x ; t)}{\partial x}$ takes into account the natural tendency of the drivers to accelerate (decelerate) if the density gradient is negative (positive), i.e. if the density in front becomes smaller (larger); therefore, it can be interpreted as proportional to the pressure gradient in the fluid describing traffic. In addition to these terms, another term proportional to $\frac{\partial^{2} v}{\partial x^{2}}$ is also added to the right hand side of the velocity equation; this tends to reduce spatial inhomogeneities of the velocity field and is usually interpreted as the analogue of the viscous dissipation term in the Navier-Stokes equation.

Thus, finally, in the fluid-dynamical approach, a complete mathematical description of the vehicular traffic on highways is provided by two equations, namely, the equation of continuity (2) and the Navier-Stokes-like velocity equation [59-62]

$$
c\left[\frac{\partial v}{\partial t}+v \frac{\partial v}{\partial x}\right]=-D \frac{\partial c}{\partial x}+\mu \frac{\partial^{2} v}{\partial x^{2}}+\frac{c}{t_{d}}\left[v_{s a f e}(c)-v\right]
$$

where $D, \mu$ and $t_{d}$ are phenomenological constants.

\subsection{Fluid-dynamical theories for multi-lane highways and city traffic}

One can describe the traffic on two-lane highways [63] by two equations each of the same form (2) and where the source term in the equation for lane 1 (lane 
2) takes into account the vehicles which enter into it from the lane 2 (lane 1) while the sink term takes into account those vehicles entering the lane 2 (lane 1) from the lane 1 (lane 2 ).

A lattice hydrodynamic theory for city traffic has been formulated recently [64]. This fluid-dynamical model is motivated by the CA model, developed by Biham et al. [21], which will be discussed in detail later in this review. Instead of generalizing the Navier-Stokes equation (17) a simpler form of the velocity equation has been assumed.

\subsection{Some recent results of the fluid-dynamical theories and their physical implications}

The fluid-dynamical model of vehicular traffic has been studied numerically by discretizing the partial differential equations (2) and (17) together with appropriate initial and boundary conditions. Both periodic boundary conditions and open boundary conditions with time-independent external flux $\alpha_{i}^{0}(t)=\beta_{i}^{0}(t)=\gamma$ have been considered.



Fig. 9. Time evolution of a density profile in the Kühne-Kerner-Konhäuser model [57-59] consisting of equations (2) and (17) with a special choice of $v_{\text {safe }}(c)$. Initially an approximately constant density profile is assumed. The figure shows that the homogeneous state is not stable, already for small deviations from a flat profile (from [13]).

In the fluid-dynamical theory, based on the equation of continuity (2) and the Navier-Stokes-like equation (17) traffic jams can appear spontaneously, even if the initial density profile $c(x ; 0)$ deviates very little from the homogeneous state (fig. 9). In order to understand the physical mechanism of the formation of local cluster of vehicles, let us consider a local increase of the density $\Delta c(x)>0$ at some location $x$. Since $d v_{\text {safe }} / d c<0$, the local increase of the density leads to a decrease of $v_{\text {safe }}$. This decrease in the safe velocity forces drivers to reduce their average velocity $v$ sharply if $\left|d v_{\text {safe }} / d c\right|$ is large enough. On the other hand, it follows from the equation of continuity that the local 
decrease of $v$ gives rise to further increase of $c$ around $x$ and, consequently, further subsequent decrease of $v(x)$ in this location. This avalanche-like process, which tends to increase the amplitude of the local fluctuation of the density around the homogeneous state, competes against other processes, like diffusion and viscous dissipation, which tend to decrease inhomogeneities.

To our knowledge, the first attempt to understand the physical mechanism of synchronized traffic within the framework of the fluid-dynamical formalism was made by Lee et al. [62] . On a finite stretch of highway, of length $L$, they installed an on-ramp and an off-ramp on the model highway with a separation of $L / 2$ between them. They chose the spatial distribution of the external flux $\phi(x)$ in equation (3) as

$$
\phi(x)=\left(2 \pi \sigma^{2}\right)^{-1 / 2} \exp \left(-x^{2} / 2 \sigma^{2}\right)
$$

with $\sigma=56.7 m$. They also assumed the form $v_{\text {safe }}(c)=v_{0}(1-c / \hat{c}) /[1+$ $\left.E(c / \hat{c})^{\theta}\right]$ for the safe velocity with adjustable parameters $v_{0}, E, \theta, \hat{c}$. Lee et al. [62] first allowed the system to reach the steady state after applying a weak time-independent flux $\alpha_{i}^{0}(t)=\beta_{i}^{0}(t)=\gamma$ and simulating the time-evolution of the traffic by solving simultaneously the equation of continuity (2) and the Navier-Stokes-like equation (17) with a specific set of chosen values for the parameters $t_{d}, D, \mu, v_{0}, E, \theta, \hat{c}$, etc. Since they chose $\gamma<\gamma_{c}$, an initially homogeneous traffic reaches a steady "free-flow" where homogeneous regions with different densities are separated from each other by narrow "transition layers" near the ramps ( no stable "free-flow" exists if $\gamma \geq \gamma_{c}$ ). Then, they applied a pulse of additional flux $\delta q$ at the on-ramp for a short duration $\delta t$. After a transient period, which depends on the parameters of the model, the system was found to settle in a limit cycle in which the local density and local flux oscillate periodically and the oscillations are localized near the on-ramp. The discontinuous change of the spatio-temporally averaged velocity induced by the localized perturbations of finite amplitude, associated hysteresis effects and the stability of the limit cycle were found to be qualitatively similar to some of the empirically observed characteristics of synchronized flow in real traffic; therefore, Lee et al. [62] identified the limit cycle observed in their theoretical investigation as the synchronized state of vehicular traffic. They drew analogy between this state and a "self-excited oscillator" [62]. However, this mechanism of the synchronized state is not yet accepted as the true and only possible explanation of the phenomena associated with the synchronized state observed empirically.

Meanwhile similar results have been obtained for a gas-kinetic based traffic model [66], also using on- and off-ramps in order to explain the transition from free-flow to synchronised states. This work was completed in a recent paper,

$\overline{7}$ A brief summary of that work can be found in [65]. 
where a phase diagram was calculated, which dependends on the on-ramp activity and the flow on the highway [67]. Summarising the recent results of the macroscopic traffic models, there seems to be evidence that on-and off-ramps play an important role for a theoretical explanation of synchronised traffic. Nevertheless some experimental features are still not captured by these approaches. E.g. the empirical results show that for synchonised traffic of type (iii) no correlations between density and flow exist [41], in contrast to the regular patterns of the oscillating states found in simulations of the macroscopic models.

Despite its success in capturing many aspects of traffic flow the fluid-dynamical approach has its limitations; for example, viscosity of traffic is not a directly measurable quantity. Nevertheless, the fluid dynamical approach is being pursued not only by some physicists but also by several members of the traffic engineering community $[68,69]$.

\section{$5 \quad$ Kinetic theories of vehicular traffic}

In the kinetic theory, traffic is treated as a gas of interacting particles where each particle represents a vehicle. The various different versions of the kinetic theory of vehicular traffic $[12,70-76]$ have been developed by modifying the kinetic theory of gases.

Recall that in the kinetic theory of gases [77] $f(\vec{r}, \vec{p} ; t) d^{3} r d^{3} p$ denotes the number of molecules which, at time $t$, have positions lying within a volume element $d^{3} r$ about $\vec{r}$ and momenta lying within the momentum-space element $d^{3} p$ about $\vec{p}$. The Boltzmann equation, which describes the time-evolution of the distribution $f(x, v ; t)$, is given by

$$
\left[\frac{\partial f}{\partial t}+\frac{\vec{p}}{m} \cdot \nabla_{r}+\vec{F} \cdot \nabla_{p}\right] f(\vec{r}, \vec{p} ; t)=\left(\frac{\partial f}{\partial t}\right)_{\text {coll }}
$$

where the symbols $\nabla_{r}$ and $\nabla_{p}$ denote gradient operators with respect to $\vec{r}$ and

$\vec{p}$, respectively, while $\vec{F}$ is the external force. The term $\left(\frac{\partial f}{\partial t}\right)_{\text {coll }}$ represents the rate of change of $f$, with time, which is caused by the mutual collisions of the molecules.

In the first of the following two subsections we present the earliest version of the kinetic theory of vehicular traffic which was introduced by Prigogine and coworkers $[70,12]$ by modifying some of the key concepts in the kinetic theory of gases and by writing down an equation analogous to the Boltzmann equation (19). In the subsequent subsection we discuss the kinetic theory developed later by Paveri-Fontana [71] to cure the defects from which the Prigogine 
theory was found to suffer.

\subsection{Prigogine model}

Suppose $f(x, v ; t) d x d v$ denotes the number of vehicles, at time $t$, between $x$ and $x+d x$, having actual velocity between $v$ and $v+d v$. In addition, Prigogine and coworkers $[70,12]$ introduced a desired distribution $f_{\text {des }}(x, v)$ which is a mathematical idealization of the goals that the population of the drivers collectively strives to achieve. The actual distribution may deviate from the desired distribution because of various possible influences, e.g., road conditions, weather conditions or interaction with other vehicles, etc. They also argued that some of these influences cease after some time while the interactions with the other vehicles persist for ever. For example, only a short stretch of the road surface may be icy and strong winds or rain may stop after a short duration; in such situations $f$ can relax to $f_{\text {des }}$ over a relaxation time $\tau_{\text {rel }}$ provided mutual interactions of the vehicles is negligibly small. On the basis of these arguments, Prigogine and coworkers [70,12] suggested that the analogue of the Boltzmann equation for the traffic should have the form

$$
\frac{\partial f}{\partial t}+v \frac{\partial f}{\partial x}=\left(\frac{\partial f}{\partial t}\right)_{r e l}+\left(\frac{\partial f}{\partial t}\right)_{i n t}
$$

where $\left(\frac{\partial f}{\partial t}\right)_{r e l}$ accounts for the relaxation of $f$ towards $f_{\text {des }}$ in the absence of mutual interactions of the vehicles while $\left(\frac{\partial f}{\partial t}\right)_{i n t}$ accounts for the changes of $f$ arising from mutual interactions among the vehicles. Note that the term $\left(\frac{\partial f}{\partial t}\right)_{i n t}$ on the right hand side of (20) may be interpreted as the analogue of the term $\left(\frac{\partial f}{\partial t}\right)_{\text {coll }}$ in the equation (19) whereas the term $\left(\frac{\partial f}{\partial t}\right)_{r e l}$ in equation (20) may be interpreted as the counterpart of the term $\vec{F} \cdot \nabla_{p} f(\vec{r}, \vec{p} ; t)$ in the equation (19).

Prigogine and coworkers wrote down an explicit form for the term $\left(\frac{\partial f}{\partial t}\right)_{i n t}$ by generalizing that for the term $\left(\frac{\partial f}{\partial t}\right)_{\text {coll }}$ in the kinetic theory of gases. We shall consider this term in the next subsection. In order to write down a simple explicit form of the relaxation term in the equation (20) they assumed that (i) the collective relaxation, which would cause the actual distribution to tend towards the desired distribution, involves only a single relaxation time $\tau_{r e l}$ so that

$$
\left(\frac{\partial f}{\partial t}\right)_{r e l}=-\frac{f-f_{d e s}}{\tau_{r e l}}
$$

and

(ii) the desired speed distribution $F_{\text {des }}(v)$ remains independent of the local 
concentration $c(x ; t)$ so that

$$
f_{\text {des }}(x, v ; t)=c(x ; t) F_{\text {des }}(v)
$$

Therefore, a more explicit form of the Boltzmann-like equation (20) in the Prigogine theory is given by

$$
\frac{\partial f}{\partial t}+v \frac{\partial f}{\partial x}=-\frac{f(x, v ; t)-c(x ; t) F_{d e s}(v)}{\tau_{r e l}}+\left(\frac{\partial f}{\partial t}\right)_{i n t}
$$

Note that, in the absence of mutual interactions of the vehicles, the distribution $f(x, v ; t)$ would relax exponentially with time. The concept of desired distribution $f_{\text {des }}(x, v ; t)$ and this scenario of collective relaxation of $f$ towards $f_{\text {des }}$ has subsequently come under severe criticism [71]. Analyzing a set of "ideal experiments" in the light of the Prigogine theory, Paveri-Fontana [71] showed that the results obtained from the Boltzmann-like equation (23) are physically unsatisfactory.

More recently, Lehmann [72] has attempted to revive the Prigogine approach by reformulating it as a semi- phenomenological theory where the distribution $f(x, v ; t)$ is assumed to follow the simpler form

$$
\frac{\partial f}{\partial t}+v \frac{\partial f}{\partial x}=-\frac{f-f_{\text {des }}(v, c)}{\tau_{\text {rel }}}
$$

and the effects of the interactions are taken into account implicitly through a density-dependent desired distribution function $f_{\text {des }}(v, c)$ which has to determined empirically.

\subsection{Paveri-Fontana model}

In order to remove the conceptual as well as mathematical drawbacks of the Prigogine model of the kinetic theory of vehicular traffic, Paveri-Fontana [71] argued that each vehicle, in contrast to the molecules in a gas, has a desired velocity towards which its actual velocity tends to "relax" in the absence of "interaction" with other vehicles. Thus, Paveri-Fontana model is based on a scenario of relaxation of the velocities of the individual vehicles rather than a collective relaxation of the distribution of the velocities.

In mathematical language, Paveri-Fontana introduced an additional phasespace coordinate, namely, the desired velocity. Suppose, $g\left(x, v, v_{d e s} ; t\right) d x d v d v_{d e s}$ denotes the number of vehicles at time $t$ between $x$ and $x+d x$, having actual velocity between $v$ and $v+d v$ and desired velocity between $v_{d e s}$ and $v_{\text {des }}+d v_{\text {des }}$. The one-vehicle actual velocity distribution function 


$$
f(x, v ; t)=\int d v_{d e s} g\left(x, v, v_{d e s} ; t\right)
$$

describes the probability of finding a vehicle between $x$ and $x+d x$ having actual velocity between $v$ and $v+d v$ at time $t$. Similarly, the one-vehicle desired velocity distribution function

$$
f_{0}\left(x, v_{d e s} ; t\right)=\int d v g\left(x, v, v_{d e s} ; t\right)
$$

describes the probability of finding a vehicle between $x$ and $x+d x$ having desired velocity between $v_{d e s}$ and $v_{d e s}+d v_{d e s}$. The local density of the vehicles $c(x ; t)$ at the position $x$ at time $t$ can be obtained from

$$
c(x ; t)=\int_{0}^{\infty} d v_{d e s} \int_{0}^{\infty} d v g\left(x, v, v_{d e s} ; t\right) .
$$

Similarly, the corresponding average actual speed $\langle v(x ; t)\rangle$ and the average desired speed $\left\langle v_{\text {des }}(x ; t)\right\rangle$ are defined as

$$
\begin{aligned}
\langle v(x ; t)\rangle & =\frac{\int_{0}^{\infty} d v_{\text {des }} \int_{0}^{\infty} d v v g\left(x, v, v_{\text {des }} ; t\right)}{c(x ; t)} . \\
\left\langle v_{\text {des }}(x ; t)\right\rangle & =\frac{\int_{0}^{\infty} d v_{\text {des }} \int_{0}^{\infty} d v v_{\text {des }} g\left(x, v, v_{\text {des }} ; t\right)}{c(x ; t)} .
\end{aligned}
$$

Finally, the local flux $J(x ; t)$ is defined as $J(x ; t)=c(x ; t)\langle v(x ; t)\rangle$.

Now let us assume that the desired velocity of each individual driver is independent of time, i.e., $d v_{d e s} / d t=0$. Of course, the drivers may also adapt to the changing traffic environment and their desired velocities may change accordingly. In principle, these features can be incorporated into the kinetic theory at the cost of increasing complexity of the formalism.

Next, let us also assume that, in the absence of interaction with other vehicles, an arbitrary vehicle reaches the desired velocity exponentially with time, i.e., $d v / d t=\left(v_{\text {des }}-v\right) / \tau$ where $\tau$ is a relaxation time. The Boltzmann-like kinetic equation for $g\left(x, v, v_{\text {des }} ; t\right)$ can be written as

$$
\left[\frac{\partial}{\partial t}+v \frac{\partial}{\partial x}\right] g+\frac{\partial}{\partial v}\left[\frac{v_{d e s}-v}{\tau} g\right]=\left(\frac{\partial g}{\partial t}\right)_{i n t}
$$

In order to write down an explicit form of the "interaction term" we have to model the interactions among the vehicles. First of all, we model the vehicles as point-like objects. We consider the scenario where a fast vehicle, when hindered by a slow leading vehicle, either passes or slows down to the velocity of the lead vehicle. Let us now make some further simplifying assumptions: 
(i) The slowing down takes place with a probability $1-P_{\text {pass }}$ where $P_{\text {pass }}$ is the probability of passing.

(ii) If the fast vehicle passes the slower leading vehicle, its own velocity remains unchanged.

(iii) The velocity of the slower leading vehicle remains unchanged, irrespective of whether the faster following vehicle passes or slows down.

(iv) The slowing down process is instantaneous, i.e., the braking time is negligibly small.

(v) It is adequate to consider only two-vehicle interactions; there is no need to consider three-vehicle (or multi-vehicle) interactions.

(vi) The postulate of "vehicular chaos", which is the analogue of the postulate of "molecular chaos" in the kinetic theory of gases, holds, so that the twovehicle distribution function $g_{2}\left(x, v, v_{d e s}, x^{\prime}, v^{\prime}, v_{d e s}^{\prime} ; t\right)$ can be approximated as a product of two one-particle distributions $g\left(x, v, v_{\text {des }} ; t\right)$ and $g\left(x^{\prime}, v^{\prime}, v_{d e s}^{\prime} ; t\right)$, i.e., $g_{2}\left(x, v, v_{\text {des }}, x^{\prime}, v^{\prime}, v_{\text {des }}^{\prime} ; t\right) \simeq g\left(x, v, v_{\text {des }} ; t\right) g\left(x^{\prime}, v^{\prime}, v_{\text {des }}^{\prime} ; t\right)$. Thus, the equation $(27)$ can be written explicitly as

$$
\begin{aligned}
{\left[\frac{\partial}{\partial t}+v \frac{\partial}{\partial x}\right] g+} & \frac{\partial}{\partial v}\left[\frac{v_{d e s}-v}{\tau} g\right] \\
= & f(x, v ; t) \int_{v}^{\infty} d v^{\prime}\left(1-P_{\text {pass }}\right)\left(v^{\prime}-v\right) g\left(x, v^{\prime}, v_{d e s} ; t\right) \\
& -g\left(x, v, v_{\text {des }} ; t\right) \int_{0}^{v} d v^{\prime}\left(1-P_{\text {pass }}\right)\left(v-v^{\prime}\right) f\left(x, v^{\prime} ; t\right)
\end{aligned}
$$

where the form of the "interaction term" on the right-hand side of the equation (28) follows from the assumptions (i)-(vi) above. The first term on the right hand side of $(28)$ describes the "gain" of probability $g\left(x, v, v_{\text {des }} ; t\right)$ from the interaction of vehicles of actual velocity $v^{\prime}$ with slower leading vehicle of actual velocity $v$ while the second term describes the loss of the probability $g\left(x, v, v_{\text {des }} ; t\right)$ arising from the interaction of vehicles of actual velocity $v$ with even slower leading vehicle of actual velocity $v^{\prime}$.

The stationary homogeneous solution $g\left(v, v_{\text {des }}\right)$ is, by definition, independent of $x$ and $t$. But, to our knowledge, so far it has not been possible to get even this solution of the Boltzmann-like integro-differential equation (28) by solving it analytically even for the simplest possible choice of the desired distribution function although numerical solutions [74,76] provide some insights into the regimes of validity of the equation (28) and gives indications as to the directions of further improvements of the Paveri-Fontana model. For example, the finite sizes of the vehicles must be taken into account at high densities. Besides, the assumption (iv) of instantaneous relaxation has also been relaxed in a more recent extension [74].

Normally passing would require more than one lane on the highway. Therefore, the models discussed so far in the context of the kinetic theory may be 
regarded, more appropriately, as quasi-one-dimensional. These neither deal explicitly with $g_{i}\left(x, v, v_{d e s} ; t\right)$ for the individual lanes (labeled by $i$ ) nor take into account the process of lane-changing. Besides, all the vehicles were assumed to be of the same type. Now, in principle, we can generalize the formalism of the kinetic theory of traffic to deal with different types of vehicles on multilane highways. Suppose $g_{i}^{a}\left(x, v, v_{\text {des }} ; t\right)$ is the distribution for vehicles of type $a$ on the $i$-th lane of the highway. Obviously, the Boltzmann-like equations for the different lanes are coupled to each other. However, one needs additional postulates to model the lane-changing rules $[78,79]$.

Very little work has been done so far on developing kinetic theories of twodimensional traffic flow which would represent, for example, traffic in cities. Suppose, for simplicity, that the network of the streets consists of north-south and east-west streets and that east-west streets allow only east-bound traffic while only north-bound traffic flow takes place along the north-south streets. Let $P_{x x}\left(P_{x y}\right)$ denote the probabilities of an east-bound vehicle passing another east-bound (north-bound) vehicle. Similarly, suppose, $P_{y x}\left(P_{y y}\right)$ denote the probabilities of a north-bound vehicle passing an east-bound (north-bound) vehicle. Let $g_{x}\left(x, y, u, u_{d e s} ; t\right)$ and $g_{y}\left(x, y, v, v_{d e s} ; t\right)$ represent the distributions for the east-bound and north-bound vehicles, respectively, where $u$ and $v$ refer to the actual their actual velocities whereas $u_{\text {des }}$ and $v_{\text {des }}$ refer to the corresponding desired velocities. The Boltzmann-like equations governing the time evolutions of these distributions are given by [80]

$$
\left[\frac{\partial}{\partial t}+u \frac{\partial}{\partial x}+v \frac{\partial}{\partial y}\right] g_{x}+\frac{\partial}{\partial u}\left[\frac{u_{d e s}-u}{\tau} g_{x}\right]=\left(\frac{\partial g_{x}}{\partial t}\right)_{x, \text { coll }}+\left(\frac{\partial g_{x}}{\partial t}\right)_{y, \text { coll }}
$$

where

$$
\begin{aligned}
\left(\frac{\partial g_{x}}{\partial t}\right)_{x, \text { coll }} & =f_{x}(x, y, u ; t) \int_{u}^{\infty} d u^{\prime}\left(1-P_{x x}\right)\left(u^{\prime}-u\right) g_{x}\left(x, y, u^{\prime}, u_{d e s} ; t\right) \\
& -g_{x}\left(x, y, u, u_{d e s} ; t\right) \int_{0}^{u} d u^{\prime}\left(1-P_{x x}\right)\left(u-u^{\prime}\right) f_{x}\left(x, y, u^{\prime} ; t\right)
\end{aligned}
$$

and

$$
\begin{aligned}
\left(\frac{\partial g_{x}}{\partial t}\right)_{y, \text { coll }} & =\delta_{u, 0} \int_{0}^{\infty} d v^{\prime} f_{y}\left(x, y, v^{\prime} ; t\right) \int_{0}^{\infty} d u^{\prime}\left(1-P_{x y}\right) u^{\prime} g_{x}\left(x, y, u^{\prime}, u_{d e s} ; t\right) \\
& -g_{x}\left(x, y, u, u_{d e s} ; t\right) \int_{0}^{\infty} d v^{\prime}\left(1-P_{x y}\right) u f_{y}\left(x, y, v^{\prime} ; t\right)
\end{aligned}
$$

The first term on the right hand side of the equation (30) describes gain of population of east-bound vehicles with velocity $u$ because of interaction with other east-bound vehicles with velocity $u^{\prime} \geq u$ while the second term describes 
the loss of population of east-bound vehicles because of interaction with eastbound vehicles of velocity $u^{\prime}<u$. The right hand side of the equation (31) is based on the assumption that when an east-bound vehicle interacts with a north-bound vehicle at a crossing, it either passes or stops.

\subsection{Derivation of the phenomenological equations of the macroscopic fluid- dynamical theories from the microscopic gas-kinetic models}

In this section we discuss the results of the attempts to derive the phenomenological equations of traffic flow in the macroscopic fluid-dynamical theories from the microscopic gas-kinetic models. Several attempts have been made so far to derive the equation of continuity and the Navier-Stokes-like equation for traffic from the corresponding Boltzmann-like equation in the same spirit in which the derivations of the equation of continuity and Navier-Stokes equation for viscous fluids from the Boltzmann-equation have been carried out. However, because of the postulate of "vehicular chaos", the equation (28) is expected to be valid only at very low densities where the correlations between the vehicles is negligibly small whereas traffic is better approximated as a continuum fluid at higher densities!

Let us define the moments

$$
m_{k, \ell}(x ; t)=\int d v \int d v_{d e s} v^{k} v_{d e s}^{\ell} g\left(x, v, v_{d e s} ; t\right)
$$

Note that $c=m_{0,0},\langle v\rangle=m_{1,0}$. Integrating the Boltzmann-like equation (28) over the actual velocities we get

$$
\frac{\partial}{\partial t} f_{0}\left(x, v_{d e s} ; t\right)+\frac{\partial}{\partial x}\left[\bar{v}\left(x, v_{d e s} ; t\right) f_{0}\left(x, v_{d e s} ; t\right)\right]=0
$$

where $\bar{v}\left(x, v_{d e s} ; t\right)$ is defined as

$$
\bar{v}\left(x, v_{d e s} ; t\right)=\frac{\int d v v g\left(x, v, v_{\text {des }} ; t\right)}{f_{0}\left(x, v_{\text {des }} ; t\right)}
$$

The equation (33) is an equation of continuity for each desired speed $v_{\text {des }}$ separately; it is consequence of the assumption that $d v_{d e s} / d t=0$, i.e., no driver changes the desired speed.

Using the Boltzmann-like equation (28) and the definition (32) we can get separate partial differential equations for the moments of $v$, moments of $v_{\text {des }}$ and the mixed moments of $v$ and $v_{\text {des }}$. Unfortunately, these lead to a hierarchy of moment equations where each evolution equation for moment of a given order involves also moments of the next higher order. In order to close this system of equations, one needs to make appropriate justifiable assumptions. 


\section{Car-following theories of vehicular traffic}

In the car-following theories $[10,11,81]$ one writes, for each individual vehicle, an equation of motion which is the analogue of the Newton's equation for each individual particle in a system of interacting classical particles. In Newtonian mechanics, the acceleration may be regarded as the response of the particle to the stimulus it receives in the form of force which includes both the external force as well as those arising from its interaction with all the other particles in the system. Therefore, the basic philosophy of the car-following theories $[10,11,81]$ can be summarized by the equation

$$
[\text { Response }]_{n} \propto[\text { Stimulus }]_{n}
$$

for the $n$-th vehicle $(n=1,2, \ldots)$. Each driver can respond to the surrounding traffic conditions only by accelerating or decelerating the vehicle. Different forms of the equations of motion of the vehicles in the different versions of the car-following models arise from the differences in their postulates regarding the nature of the stimulus (i.e., "behavioural force" or a "generalized force" [82]). The stimulus may be composed of the speed of the vehicle, the difference in the speeds of the vehicle under consideration and its lead vehicle, the distanceheadway, etc., and, therefore, in general,

$$
\ddot{x}_{n}=f_{s t i}\left(v_{n}, \Delta x_{n}, \Delta v_{n}\right)
$$

where the function $f_{\text {sti }}$ represents the stimulus received by the $n$-th vehicle. Different versions of the car-following models model the function $f_{\text {sti }}$ differently. In the next two subsections we discuss two different conceptual frameworks for modeling $f_{\text {sti }}$.

\subsection{Follow-the-leader model}

In the earliest car-following models $[83,84]$ the difference in the velocities of the $n$-th and $(n+1)$-th vehicles was assumed to be the stimulus for the $n$-th vehicle 8 . In other words, it was assumed that every driver tends to move with the same speed as that of the corresponding leading vehicle so that

$$
\ddot{x}_{n}(t)=\frac{1}{\tau}\left[\dot{x}_{n+1}(t)-\dot{x}_{n}(t)\right]
$$

where $\tau$ is a parameter that sets the time scale of the model. Note that $1 / \tau$ in the equation (37) can be interpreted as a measure of the sensitivity coefficient

$\overline{8}$ In the following we label the vehicles in driving direction such that the $(n+1)$-th vehicle is in front of the $n$-th vehicle. 
$\mathcal{S}$ of the driver; it indicates how strongly the driver responds responds to unit stimulus. According to such models (and their generalizations proposed in the fifties and sixties) the driving strategy is to follow the leader and, therefore, such car-following models are collectively referred to as the follow-the-leader model.

Pipes [84] derived the equation (37) by differentiating, with respect to time, both sides of the equation

$$
\Delta x_{n}(t)=x_{n+1}(t)-x_{n}(t)=(\Delta x)_{s a f e}+\tau \dot{x}_{n}(t)
$$

which encapsulates his basic assumption that (a) the higher is the speed of the vehicle the larger should be the distance-headway, and, (b) in order to avoid collision with the leading vehicle, each driver must maintain a safe distance $(\Delta x)_{s a f e}$ from the leading vehicle.

It has been argued [85] that, for a more realistic description, the strength of the response of a driver at time $t$ should depend on the stimulus received from the other vehicles at time $t-T$ where $T$ is a response time lag. Therefore, generalizing the equation (37) one would get [85]

$$
\ddot{x}_{n}(t+T)=\mathcal{S}\left[\dot{x}_{n+1}(t)-\dot{x}_{n}(t)\right]
$$

where the sensitivity coefficient $\mathcal{S}$ is a constant independent of $n$.

According to the equations (37) and (39), a vehicle would accelerate or decelerate to acquire the same speed as that of its leading vehicle. This implies that, as if, slower following vehicle are dragged by their faster leading vehicle. In these linear dynamical models the acceleration response of a driver is completely independent of the distance-headway. Therefore, this oversimplified equation, fails to account for the clustering of the vehicles observed in real traffic. Moreover, since there is no density-dependence in this dynamical equation, the fundamental relation cannot be derived from this dynamics. In order to make the model more realistic, we now assume [86] that the closer is the $n$-th vehicle to the $(n+1)$-th the higher is the sensitivity of the driver of the $n$-th car. In this case, the dynamical equation (39) is further generalized to

$$
\ddot{x}_{n}(t+T)=\frac{\kappa}{\left[x_{n+1}(t)-x_{n}(t)\right]}\left[\dot{x}_{n+1}(t)-\dot{x}_{n}(t)\right]
$$

where $\kappa$ is a constant. An even further generalization of the the model can be achieved $[87,88]$ by expressing the sensitivity factor for the $n$-th driver as

$$
\mathcal{S}_{n}=\frac{\kappa\left[v_{n}(t+\tau)\right]^{m}}{\left[x_{n+1}(t)-x_{n}(t)\right]^{\ell}}
$$


where $\ell$ and $m$ are phenomenological parameters to be fixed by comparison with empirical data. These generalized follow-the-leader models lead to coupled non-linear differential equations for $x_{n}$. Thus, in this "microscopic" theoretical approach, the problem of traffic flow reduces to problems of nonlinear dynamics.

So far as the stability analysis is concerned, there are two types of analysis that are usually carried out. The local stability analysis gives information on the nature of the response offered by the following vehicle to a fluctuation in the motion of its leading vehicle. On the other hand, the manner in which a fluctuation in the motion of any vehicle is propagated over a long distance through a sequence of vehicles can be obtained from an asymptotic stability analysis.

From experience with real traffic we know that drivers often observe not only the leading vehicle but also a few other vehicles ahead of the leading vehicle. For example, the effect of the leading vehicle of the leading vehicle can be incorporated in the same spirit as the effect of "next-nearest-neighbour" in various lattice models in statistical mechanics. A linear dynamical equation, which takes into account this "next-nearest-neighbour" within the framework of the follow-the-leader model, can be written as [81]

$$
\ddot{x}_{n}(t+T)=\mathcal{S}^{(1)}\left[\dot{x}_{n+1}(t)-\dot{x}_{n}(t)\right]+\mathcal{S}^{(2)}\left[\dot{x}_{n+2}(t)-\dot{x}_{n}(t)\right]
$$

where $\mathcal{S}^{(1)}$ and $\mathcal{S}^{(2)}$ are two phenomenological response coefficients.

The weakest point of these theories is that these involve several phenomenological parameters which are determined through "calibration", i.e., by fitting some predictions of the theory with the corresponding empirical data. Besides, extension of these models to multi-lane traffic is difficult since every driver is satisfied if he/she can attain the desired speed!

\subsection{Optimal velocity models}

We can express the driving strategy of the driver in the car-following models in terms of mathematical symbols by writing

$$
\ddot{x}_{n}(t)=\frac{1}{\tau}\left[V_{n}^{\text {desired }}(t)-v_{n}(t)\right]
$$

where $V_{n}^{\text {desired }}(t)$ is the desired speed of the $n$-th driver at time $t$. In all followthe-leader models mentioned above the driver maintains a safe distance from the leading vehicle by choosing the speed of the leading vehicle as his/her own desired speed, i.e., $V_{n}^{\text {desired }}(t)=\dot{x}_{n+1}$. 
An alternative possibility has been explored in recent works based on the carfollowing approach [89-104]. This formulation is based on the assumption that $V_{n}^{\text {desired }}$ depends on the distance-headway of the $n$-th vehicle, i.e., $V_{n}^{\text {desired }}(t)=$ $V^{\text {opt }}\left(\Delta x_{n}(t)\right)$ so that

$$
\ddot{x}_{n}(t)=\frac{1}{\tau}\left[V^{\text {opt }}\left(\Delta x_{n}(t)\right)-v_{n}(t)\right]
$$

where the so-called optimal velocity function $V^{\text {opt }}\left(\Delta x_{n}\right)$ depends on the corresponding instantaneous distance-headway $\Delta x_{n}(t)=x_{n+1}(t)-x_{n}(t)$. In other words, according to this alternative driving strategy, the $n$-th vehicle tends to maintain a safe speed that depends on the relative position, rather than relative velocity, of the $n$-th vehicle. In general, $V^{\text {opt }}(\Delta x) \rightarrow 0$ as $\Delta x \rightarrow 0$ and must be bounded for $\Delta x \rightarrow \infty$. For explicit calculations, one has to postulate a specific functional form of $V^{o p t}(\Delta x)$. Car-following models along this line of approach have been introduced by Bando et al. [89,90]. For obvious reasons, these models are usually referred to as optimal velocity (OV) models.

Since the equations of motion in the follow-the-leader models involve only the velocities, and not positions, of the vehicles these can be formulated as essentially first order differential equations (for velocities) with respect to time. In contrast, since the equations of motion in the OV model involve the positions of the vehicles explicitly, the theoretical problems of this model are formulated mathematically in terms of second order differential equations (for the positions of the vehicles) with respect to time $[89,90]$.

The simplest choice for $V^{o p t}(\Delta x)$ is $[91,92]$

$$
V^{o p t}(\Delta x)=v_{\max } \Theta(\Delta x-d)
$$

where $d$ is a constant and $\Theta$ is the Heavyside step function. According this form of $V^{o p t}(\Delta x)$, a vehicle should stop if the corresponding distance-headway is less than $d$; otherwise, it can accelerate so as to reach the maximum allowed velocity $v_{\max }$. A somewhat more realistic choice $[92,96]$ is

$$
V^{\text {opt }}(\Delta x)= \begin{cases}0 & \text { for } \quad \Delta x<\Delta x_{A} \\ f \Delta x & \text { for } \quad \Delta x_{A} \leq \Delta x \leq \Delta x_{B} \\ v_{\max } & \text { for } \quad \Delta x_{B}<\Delta x\end{cases}
$$

The main advantage of the forms (45) and (46) of the OV function is that exact analytical calculations, e.g. in the jammed region, are possible [92]. Although (45) and (46) may not appear very realistic, they capture several key features of more realistic forms of OV function [89], e.g.,

$$
V^{o p t}(\Delta x)=\tanh \left[\Delta x-\Delta x_{c}\right]+\tanh \left[\Delta x_{c}\right]
$$


for which analytical calculations are very difficult. For the convenience of numerical investigation, the dynamical equation (44) for the vehicles in the OV model has been discretized and, then, rewritten as a difference equation [104].



Fig. 10. Fundamental diagram of the OV model. The solid line shows the OV function and the dots simulation data. One can distinguish five different density regimes with respect to the stable stationary state (from [92]).

The main question addressed by the OV model is the following: what is the condition for the stability of the homogeneous solution

$$
x_{n}^{h}=b n+c t
$$

where $b=(\Delta x)_{a v}=L / N$ is the constant average spacing between the vehicles and $c$ is the constant velocity. It is not difficult to argue that, in general, in the OV models the homogeneous flow becomes unstable when $\left.\frac{\partial V^{\text {opt }}}{\partial \Delta x}\right|_{\Delta x=b}>\frac{2}{\tau}$ [89].

One can distinguish five different density regimes with respect to the stability of microscopic states (see Fig. 10). At low and at high densities the homogeneous states are stable. For intermediate densities one can distinguish three regimes where jammed states exist. In region III the jammed state is stable whereas in regions II and IV both homogeneous and jammed states form stable structures. Beyond the formation of jams also hysteresis effects have been observed. Thus the OV model is able to reproduce many aspects of experimental findings.

Modified Korteweg-de Vries (KdV) equation has been derived from the equation (44) in a special regime of the parameters [93] and the relations between its kink solutions and traffic congestion have been elucidated [99]. A generalization to two-lane traffic can be found in [105]. In order to account for 
traffic consisting of two different types of vehicles, say, cars and trucks, Mason et al. [97] generalized the formulation of Bando et al. [89] by replacing the constant $\tau$ by $\tau_{n}$ so that

$$
\ddot{x}_{n}(t)=\frac{1}{\tau_{n}}\left[V_{n}^{o p t}(t)-v_{n}(t)\right]
$$

where $\tau_{n}$ now depends on whether the $n$-th vehicle is a car or a truck. Since a truck is expected to take longer to respond than a car we should assign larger $\tau$ to trucks and smaller $\tau$ to cars. Some other mathematically motivated generalizations of the OV model have also been considered $[95,98,100]$.

As mentioned earlier, drivers often receive stimulus not only from the leading vehicle but also a few other vehicles ahead of the leading vehicle. One possible way to generalize the OV models for taking into account such multi-vehicle interactions [102] is to write the dynamical equations as

$$
\ddot{x}_{n}=\sum_{j=1}^{m} \mathcal{S}_{j}\left[V^{\text {opt }}\left(\frac{x_{n+j}-x_{n}}{j}\right)-v_{n}\right]
$$

where $\mathcal{S}_{j}$ are sensitivity coefficients; one of the commonly used explicit forms of the optimal velocity function, for example (47) can be chosen for that of the function $V^{\text {opt }}$ in (50).

Before concluding our discussions on the OV models let us mention a very simple model [94] where one assumes that

$$
\ddot{x}_{n}(t)=\left\{\begin{array}{lll}
a & \text { for } & \Delta x_{n} \geq \Delta x_{c} \\
-a & \text { for } & \Delta x_{n}<\Delta x_{c} .
\end{array}\right.
$$

with $a>0$. Obviously, $\Delta x_{c}$ may be interpreted as the safety distance. Moreover, a restriction $v_{\text {min }} \leq v_{n} \leq v_{\max }$ is imposed on the allowed velocities of the vehicles by introducing the allowed minimum and maximum velocities $v_{\min }$ and $v_{\max }$, respectively. Note that in this oversimplified model $\ddot{x}_{n}$ depends on the corresponding distance-headway and, therefore, has some apparent similarities with the OV models. But, unlike the more general OV models, in this model $\ddot{x}_{n}$ does not depend on its instantaneous velocity. The main reason for considering such a oversimplified scenario is that velocity of the propagation of jams can be calculated analytically.

Some ideas of the OV model has been utilized by Mahnke and Pieret [106] in their master equation approach to the study of jam dynamics. They assumed that, at a time, only one vehicle can go into or come out of a jam; this, naturally, does not take into the merging or splitting of jams. Under this assumption, the master equation (see e.g. App. F, equation (F.1)) governing the probability distribution $P(n ; t)$ of the jam sizes $n$ is given by 


$$
\begin{aligned}
\frac{d P(n ; t)}{d t} & =W_{+}(n-1) P(n-1 ; t)+W_{-}(n+1) P(n+1 ; t) \\
& -\left[W_{+}(n)+W_{-}(n)\right] P(n ; t)
\end{aligned}
$$

where $W_{+}$and $W_{-}$, are the "growth" and "decay" transition rates, respectively. It has been argued $[106,107]$ that $W_{-}(n)=1 / \tau=$ constant since a vehicle would require a constant average time $\tau$ to come out of a jam. However, $W_{+}(n)$ would depend on $V^{o p t}(\Delta x)$ since the time taken by a free-flowing vehicle immediately behind a jam to get into the jam would depend on $\Delta x$ as well as on $V^{o p t}(\Delta x)$ although the actual expression of $W_{+}(n)$ may be complicated in a reasonable ansatz [107]. Moreover, several assumptions of the model will have to be relaxed before the results of this approach can be compared with those from real traffic.

Before concluding this section we would like to emphasize that while formulating the dynamical equations for updating the velocities and positions of the vehicles in any "microscopic" theory the following points should be considered: (i) in the absence of any disturbance from the road conditions and interactions with other vehicles, a driver tends to drive with a desired velocity $v_{\text {des }}$; if the actual current velocity of the vehicle $v(t)$ is smaller (larger) than $v_{\text {des }}$, the vehicle accelerates (decelerates) so as to approach $v_{\text {des }}$.

(ii) In a freely-flowing traffic, even when a driver succeeds in attaining the desired velocity $v_{d e s}$, the velocity of the vehicle fluctuates around $v_{\text {des }}$ rather than remaining constant in time.

(iii) The interactions between a pair of successive vehicles in a lane cannot be neglected if the gap between them is shorter than $v_{d e s}$; in such situations the following vehicle must decelerate so as to avoid collision with the leading vehicle.

Clearly, the reliability of the predictions of the OV model depends on the appropriate choice of the optimal velocity function.

\section{Coupled-map lattice models of vehicular traffic}

Recall that, in the car-following models, space is assumed to be a continuum and time is represented by a continuous variable $t$. Besides, velocity and acceleration of the individual vehicles are also real variables. However, most often, for numerical manipulations of the differential equations of the car-following models, one needs to discretize the continuous variables with appropriately chosen grids. In contrast, in the coupled-map lattice approach [108], one starts with a discrete time variable and, the dynamical equations for the individual vehicles are formulated as discrete dynamical maps that relate the state variables at time $t$ with those at time $t+1$, although position, 
velocity and acceleration are not restricted to discrete integer values. The unit of time in this scheme (i.e., one time step) may be interpreted as the reaction time of the individual drivers as the velocity of of a vehicle at the time step $t$ depends on the traffic conditions at the preceding time step $t-1$.

Keeping in mind the general points raised at the end of the preceding section regarding the formulation of the dynamical equations for updating the velocities and positions of the vehicles, the general forms of the dynamical maps in the coupled-map lattice models can be expressed as:

$$
\begin{aligned}
v_{n}(t+1) & =\operatorname{Map}_{n}\left[v_{n}(t), v_{\text {des }}, \Delta x_{n}(t)\right] \\
x_{n}(t+1) & =v_{n}(t)+x_{n}(t)
\end{aligned}
$$

where $v_{d e s}$ is a desired velocity. In general, the dynamical map $\operatorname{Map}\left[v_{n}(t), v_{d e s}, \Delta x_{n}(t)\right]$ takes into account the velocity $v_{n}(t)$ and the distance-headway $\Delta x_{n}(t)$ of the $n$-th vehicle at time $t$ for deciding the velocity $v_{n}(t+1)$ at time $t+1$. The effects of the interactions among the vehicles enter into the dynamical updating rules (53), (54) only through the distance-headway $\Delta x_{n}$.

\subsection{The model of Yukawa and Kikuchi}

Yukawa and Kikuchi [109-111] have studied coupled-map models based on the map

$$
v(t+1)=F(v(t)):=\gamma v(t)+\beta \tanh \left(\frac{v^{F}-v(t)}{\gamma}\right)+\epsilon
$$

for the uninfluenced motion of a single vehicle. $v^{F}$ is the preferred velocity of the vehicle and $\beta, \gamma, \delta$ and $\epsilon$ are parameters. For $\gamma$ close to 1 the map becomes chaotic, but acceleration and deceleration are approximately constant far from $v^{F}$. Their magnitude is determined by the parameter $\beta . \epsilon$ controls the difference of the acceleration and deceleration capabilities. Although the model is deterministic, fluctuations in the velocity are introduced through deterministic chaos. These fluctuations around $v^{F}$ are determined by the parameter $\delta$.

If there is more than one vehicle on the road one needs an additional deceleration mechanism to avoid collisions. This can be achieved by introducing a deceleration map. Assuming that deceleration is dominated by the headway, two models have been studied in [109]. In model A, the deceleration map describes a sudden braking process. If the front-bumper to front-bumper distance $\Delta x_{n}$ to the next vehicle ahead is less than the current velocity $v_{n}(t)$ of the following vehicle, then the velocity is reduced to $\Delta x_{n}-l$ where $l$ is the length of the vehicles. The corresponding map is $B\left(\Delta x_{n}(t)\right)=\Delta x_{n}(t)-l$. 
Model B has a more complex deceleration map:

$$
\begin{aligned}
v_{n}(t+1)=G\left(\Delta x_{n}(t), v_{n}(t)\right):= & \frac{F\left(v_{n}(t)\right)-v_{n}(t)}{(\alpha-1) v_{n}(t)}\left[\Delta x_{n}(t)-l-v_{n}(t)\right] \\
& \left(\text { for } v_{n}(t) \leq \Delta x_{n}(t)-l \leq \alpha v_{n}(t)\right) .
\end{aligned}
$$

The parameter $\alpha$ determines the range within which the deceleration map $G(\Delta x, v)$ is used. For headways less than $\alpha v_{n}(t)$ the map $G$ is used instead of $F(v)$. Note that $G(\Delta x, v=\Delta x-l)=\Delta x-l$ and $G(\Delta x, v=(\Delta x-l) / \alpha)=$ $F(v=(\Delta x-l) / \alpha)$, i.e. $G$ interpolates between the free-motion map $F$ and the sudden braking map of model A. The full velocity map of Model B is thus given by

$$
\operatorname{Map}_{n}\left(v_{n}(t), \Delta x_{n}(t)\right)= \begin{cases}F\left(v_{n}(t), v_{n}^{F}\right) & \text { for } \alpha v_{n}(t) \leq \Delta x_{n}(t) \\ G\left(\Delta x_{n}(t), v_{n}(t)\right) & \text { for } \left.v_{n}(t) \leq \Delta x_{n}(t) \leq \alpha v_{n}(t)\right) \\ B\left(\Delta x_{n}(t)\right) & \text { for } \left.\Delta x_{n}(t) \leq v_{n}(t)\right)\end{cases}
$$

Local measurements of the flow for a system of vehicles with different preferred velocities $v_{n}^{F}$ produce a fundamental diagram of inverse- $\lambda$ shape (see Fig. 4A) [111]. Here the non-uniqueness of the flow has a simple explanation. Due to the different $v_{n}^{F}$, platoons form behind the slowest vehicles. Whenever such a platoon passes the measurement region, a flow value on the lower branch is recorded. Otherwise, the flow corresponds to the upper branch.

Measurements of the power spectral density of temporal density fluctuations, i.e. the Fourier transform of the time-series of local densities, show a $1 / f^{\alpha_{-}}$ behaviour with $\alpha \approx 1.8$ in the free-flow regime. Due to the deterministic dynamics, the system evolves into a state with power-law fluctuations. In $[110,111]$ it has been suggested that the origin of the $1 / f^{\alpha}$-fluctuations is the power-law distribution $\left(\propto 1 /(\Delta x)^{3.0}\right)$ of the headways $\Delta x$, since these are related to density waves. The occurence of jams destroys long-time correlations since vehicles loose their memory of current fluctuations when they are forced to stop in a jam [110]. Therefore no $1 / f^{\alpha}$-behaviour can be observed in the jammed regime.

In [112] a coupled-map model based on optimal-velocity functions has been introduced by discretizing the time variable of the OV model (see Sec. 6.2). This allows to study systems with open boundaries and multilane systems. Furthermore a multiplicative random noise can be imposed in the velocity update so that the velocity map is given by

$$
v(t+1)=\left[v(t)+\alpha\left(V_{\text {opt }}(\Delta x)-v(t)\right)\right]\left(1+f_{\text {noise }} \xi\right)
$$


where $\xi \in[-1 / 2,1 / 2]$ is a uniform random variable and $f_{\text {noise }}$ the noise level.

\subsection{The model of Nagel and Herrmann}

Nagel and Herrmann (NH) [113] have introduced a coupled-map model which is related to the continuum limit of the Nagel-Schreckenberg cellular automata model (see Sec. 8). A generalization of the NH model has later been presented in [114]. Vehicles are characterized by a maximal velocity $v_{\max }$ and a safety distance $\alpha$. The velocity map for the NH model is given by

$$
v_{n}(t+1)= \begin{cases}\max \left(\Delta x_{n}(t)-\delta, 0\right) & \text { for } v_{n}(t)>\Delta x_{n}(t)-\alpha \\ \min \left(v_{n}(t)+a, v_{\max }\right) & \text { for } v_{n}(t)<\Delta x_{n}(t)-\beta\end{cases}
$$

In the velocity update step, vehicles which have a headway $\Delta x$ smaller than the safety distance $\alpha$ decelerate. The headway distance after deceleration is determined by the parameter $\delta$. Vehicles which have a large enough headway, on the other hand, accelerate. The acceleration coefficient $a$ is determined by $a=a_{\max } \max \left(1, \Delta x_{n}(t) / \gamma\right)$.

Since the dynamics of the model is deterministic, the behaviour depends strongly on fluctuations of the initial state [114]. For equidistant starting positions of the vehicles the fundamental diagram consists of two linear branches with maximum flow $f\left(c_{m}\right)=v_{\max } c_{m}$ at density $c_{m}=1 /\left(v_{\max }+\beta\right)$. For homogeneous starting positions the system is free-flowing up to a critical density $c_{\text {crit }}$. Beyond this density free-flowing and congested areas coexist.

\subsection{The model of Krauss, Wagner and Gawron}

Krauss et al. $[115,116]$ introduced a whole class of stochastic models by considering necessery conditions for the collision-free motion of vehicles. The models are continuous in space and discrete in time.

The vehicles are characterized by a maximum velocity $v_{\max }$, their acceleration and deceleration capabilities $a(v)$ and $b(v)$, respectively, and their length $l$ which will be taken to be $l=1$ in the following. Then the update rules for the velocity $v$ and the space coordinate $x$ of each vehicle are as follows:

Step 1: Determine desired velocity.

$$
v_{\text {des }}=\min \left[v_{\max }, v+a(v), v_{\text {safe }}\right]
$$


Step 2: Randomization.

$$
v=\max \left[0, \operatorname{rand}\left(v_{\text {des }}-a, v_{d e s}\right)\right]
$$

Step 3: Vehicle movement.

$$
x \rightarrow x+v
$$

Here $\operatorname{rand}\left(v_{1}, v_{2}\right)$ denotes a random number uniformly distributed in the interval $\left[v_{1}, v_{2}\right)$ and $v_{\text {safe }}$ is a velocity which guarantees collision motion of the vehicles. It is given explicitly by

$$
v_{\text {safe }}=v_{p}+b(\hat{v}) \frac{g-v_{p}}{\hat{v}+b(\hat{v})} .
$$

where $v_{p}$ is the velocity of the preceding vehicle located at $x_{p}$ and $g=x_{p}-x-1$ is the headway, i.e. the distance to the preceding vehicle.

In the simplest case the acceleration and deceleration capabilities do not depend on the velocity, i.e. $a(v)=a=$ const and $b(v)=b=$ const. The behaviour of the model can be classified in three different families (see Fig. 11).



Fig. 11. Schematic sketch of the three different classes of models in the KWG model.

The three families of models sketched schematically in Fig. 11 can be characterized as follows:

\section{Class I: High acceleration}

Here no spontaneous jamming exists. For $a \rightarrow v_{\max }$ and $b \gg 1$ the behaviour is similar to that of a cellular automata model without velocity memory introduced in [117] which is closely related to the Kasteleyn model [118] of statistical physics. It can also be interpreted as 5-vertex model [119]. Another model belonging to this class has been introduced by Fukui and Ishibashi [120] (see Sec. 11.1).

\section{Class II: High acceleration - low deceleration}

The outflow from a jam is identical to the maximal possible flow. The jamming transition is not a true phase transition, but rather a crossover. The limit 
$b \rightarrow \infty, a=1$ corresponds to a continuum version of the Nagel-Schreckenberg cellular automata model [20] which will be introduced in the next section.

Class III: Low acceleration - low deceleration

These models exhibit phase separation and metastability. The jamming transition is of first order. The outflow from a jam is not maximal. For $a \ll v_{\max }$ and $b \ll v_{\max }$ the model is closely related to the Gipps model [88] discussed in Sec. 6.1. Other models belonging to this class are the Kerner-Konhäuser model (see Sec. 4.4), the optimal-velocity model (Sec. 6.2) and the models with slow-to-start rules which will be introduced in Sec. 9.1.2.

On a macroscopic level, classes I, II and III can be distinguished by the ordering of the densities $c_{f}$ and $c_{c}$, where $c_{f}$ is the density of the outflow from a jam and $c_{c}$ is the density where homogeneous flow becomes unstable [116]. For $c_{c}>c_{f}$ the outflow from a jam is stable and the system phase-separates into free-flow and jammed regions. Furthermore, metastable states can be found. This is the type of the behaviour found in class III. For $c_{c}<c_{f}$, on the other hand, the outflow from a jam is unstable and no metastable states or phase separation can be found. This is the typical behaviour of classes I and II. These classes can further be distinguished since in class I one does not find any structure formation, like spontaneous jamming, in contrast to class II.

A related model has been studied before by Migowsky et al. [121]. In this model vehicles are also characterized by a maximum velocity $v_{\max }$ and a bounded acceleration capability $\left(-b_{\max }<\ddot{x}<a_{\max }\right)$ which determines the safety distance $d_{s}$ necessary to avoid accidents. The investigations in [121] focussed on the effect of so-called driving strategies. These strategies are characterized by a vector $\left(f_{v}, f_{a}, f_{s}\right)$, where $f_{v}, f_{a}$ and $f_{s}$ are the fraction of the vehicle's maximal velocity, acceleration and safety distance actually used?' respectively. This can lead to the possibility of accidents and allows to study the number of crashes as a function of the driving strategies.

In [121] different strategies have been compared. Furthermore, dynamical changes of strategies can be introduced which allow the drivers to adapt to the local traffic conditions. In general this leads to a decrease in the number of accidents, jams and fuel consumption, but at high densities the flow is reduced compared to the case of fixed strategies.

9 In other words, the maximum velocity is given by $v_{\max }^{(n)}=f_{v}^{(n)} v_{\max }$ etc. 


\section{Nagel-Schreckenberg cellular automata model of vehicular traffic on highways}

In general, CA are idealization of physical systems in which both space and time are assumed to be discrete and each of the interacting units can have only a finite number of discrete states. Note that for a discretization of differential equations, e.g. those of the hydrodynamic approach, space and time variables are discrete, but the state variable still is continuous. The concept of CA was introduced in nineteen fifties by von Neumann while formulating an abstract theory of self-replicating computing machines [122]. However, it received the attention of a wider audience in the nineteen seventies through Conway's game of life [123]. The family of one-dimensional CA was studied systematically, in the nineteen eighties, from the point of view of dynamical systems and popularized by Wolfram $[18,124]$. Since then the concept of CA has been applied to model a wide variety of systems [125-127]. To our knowledge, the first CA model for vehicular traffic was introduced by Cremer and Ludwig [128].

In the CA models of traffic the position, speed, acceleration as well as time are treated as discrete variables. In this approach, a lane is represented by a one-dimensional lattice. Each of the lattice sites represents a "cell" which can be either empty or occupied by at most one "vehicle" at a given instant of time (see Fig. 12). At each discrete time step $t \rightarrow t+1$, the state of the system is updated following a well defined prescription (a summary of various possible different schemes of updating is given in Appendix A). The computational efficiency of the discrete CA models is the main advantage of this approach over the car-following and coupled-map lattice approaches.



Fig. 12. A typical configuration in the NaSch model. The number in the upper right corner is the speed of the vehicle.

In the NaSch model, the speed $v$ of each vehicle can take one of the $v_{\max }+1$ allowed integer values $v=0,1, \ldots, v_{\max }$. Suppose, $x_{n}$ and $v_{n}$ denote the position and speed, respectively, of the $n$-th vehicle. Then, $d_{n}=x_{n+1}-x_{n}$, is the gap in between the $n$-th vehicle and the vehicle in front of it at time $t$. At each time step $t \rightarrow t+1$, the arrangement of the $N$ vehicles on a finite lattice of length $L$ is updated in parallel according to the following "rules":

Step 1: Acceleration. If $v_{n}<v_{\max }$, the speed of the $n$-th vehicle is increased 
by one, but $v_{n}$ remains unaltered if $v_{n}=v_{\max }$, i.e.

$$
v_{n} \rightarrow \min \left(v_{n}+1, v_{\max }\right)
$$

Step 2: Deceleration (due to other vehicles). If $d_{n} \leq v_{n}$, the speed of the $n$-th vehicle is reduced to $d_{n}-1$, i.e.,

$$
v_{n} \rightarrow \min \left(v_{n}, d_{n}-1\right)
$$

Step 3: Randomization. If $v_{n}>0$, the speed of the $n$-th vehicle is decreased randomly by unity with probability $p$ but $v_{n}$ does not change if $v_{n}=0$, i.e.,

$$
v_{n} \rightarrow \max \left(v_{n}-1,0\right) \quad \text { with probability } p
$$

Step 4: Vehicle movement. Each vehicle is moved forward according to its new velocity determined in Steps 1-3, i.e.

$$
x_{n} \rightarrow x_{n}+v_{n}
$$

The NaSch model is a minimal model in the sense that all the four steps are necessary to reproduce the basic features of real traffic; however, additional rules need to be formulated to capture more complex situations. The step 1 reflects the general tendency of the drivers to drive as fast as possible, if allowed to do so, without crossing the maximum speed limit. The step 2 is intended to avoid collision between the vehicles. The randomization in step 3 takes into account the different behavioural patterns of the individual drivers, especially, nondeterministic acceleration as well as overreaction while slowing down; this is crucially important for the spontaneous formation of traffic jams. Even changing the precise order of the steps of the update rules stated above would change the properties of the model. E.g. after changing the order of steps 2 and 3 there will be no overreactions at braking and thus no spontaneous formation of jams. The NaSch model may be regarded as stochastic CA [18]. In the special case $v_{\max }=1$ the deterministic limit of the NaSch model is equivalent to the CA rule 184 in Wolfram's notation [18] and some abstract extensions of this CA-184 rules [129] have been studied in the more general context of complex dynamics and particle flow.

Why should the updating be done in parallel, rather than in random sequential manner, in traffic models like the NaSch model? In contrast to a random sequential update, parallel update can lead to a chain of overreactions. Suppose, a vehicle slows down due the randomization step. If the density of vehicles is large enough this might force the following vehicle also to brake in the deceleration step. In addition, if $p$ is larger than zero, it might brake even further in Step 3. Eventually this can lead to the stopping of a vehicle, thus creating 
a jam. This mechanism of spontaneous jam formation is rather realistic and cannot be modeled by the random sequential update.

The update scheme of the NaSch model is illustrated with a simple example in Fig. 13.

\section{Comflguration at time t:}

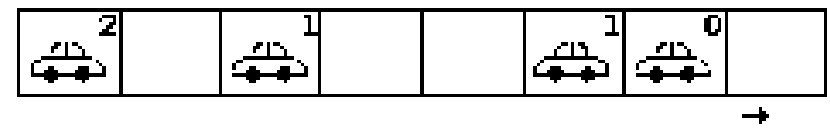

a) Acceleration:

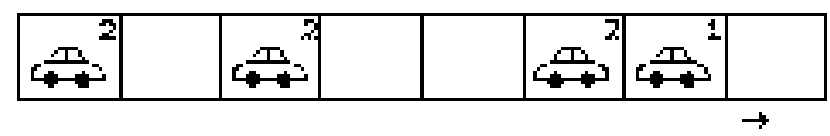

b) Braking:



c) Randomizatlon $(p=1 / 3)$ :



\section{d) Droing (= comflguration at time $t+1)$ :}



Fig. 13. Step-by-step example for the application of the update rules. We have assumed $v_{\max }=2$ and $p=1 / 3$. Therefore on average one third of the cars qualifying will slow down in the randomization step.

Space-time diagrams showing the time evolutions of the NaSch model demonstrate that no jam is present at sufficiently low densities, but spontaneous fluctuations give rise to traffic jams at higher densities (Fig. 14(a)). From the Fig. 14(b) it should be obvious that the intrinsic stochasticity of the dynamics [20], arising from non-zero $p$, is essential for triggering the jams [20,113]. For a realistic description of highway traffic [20], the typical length of each cell should be about $7.5 \mathrm{~m}$ which is the space occupied by a vehicle in a dense jam. When $v_{\max }=5$ each time step should correspond to approximately 1 sec of 



Fig. 14. Typical space-time diagrams of the NaSch model with $v_{\max }=5$ and (a) $p=0.25, c=0.20$, (b) $p=0, c=0.5$. Each horizontal row of dots represents the instantaneous positions of the vehicles moving towards right while the successive rows of dots represent the positions of the same vehicles at the successive time steps.

real time which is of the order of the shortest relevant timescale in real traffic, namely the reaction time of the drivers.

Almost all the models of traffic considered in this review, including the NaSch model, have been formulated in such a way that no accident between successive vehicles is possible. However, accident of the vehicles is possible if the condition for safe driving is relaxed. For example, Boccara et al. [130] replaced the update rule of the NaSch model by the rule

$$
\text { if } v_{n+1}(t)>0 \text { then } x_{n}(t+1)=x_{n}(t)+v_{n}(t+1)+\Delta v
$$

where $\Delta v$ is a Bernoulli random variable which takes the value 1 with probability $p_{\text {careless }}$ and zero with the probability $1-p_{\text {careless. }}$. The probability $P_{a c}$ of accident per vehicle per time step is a non-monotonic function of the vehicle density $c[130,131]$.

\subsection{Relation with other models}

\subsubsection{Relation with totally asymmetric simple exclusion process}

Now we point out the similarities and differences between the $v_{\max }=1$ limit of the NaSch model and the totally asymmetric simple exclusion process (TASEP) which is the simplest prototype model of interacting systems driven far from equilibrium $[16,14,15]$. In the TASEP (Fig. 15) a randomly chosen particle can move forward, by one lattice spacing, with probability $q$ if the lattice site immediately in front of it is empty. It corresponds to a Kawasaki dynamics [132] for exchange of a charged particle and hole on nearest-neighbour lattice sites at infinite temperature and in the presence of an infinite electric field [133] (see Appendix B for some technical aspects of TASEP). Several 
different generalized variants of the TASEP have been considered. For example, in the $k$-hop model [134] a particle can exchange its position with the nearest-hole on its right with probability $q$, provided the separation of the two sites under consideration is not more than $k$ lattice spacings. The $k$-hop model reduces to the TASEP in the special case $k=1$.



Fig. 15. ASEP with periodic boundary conditions.

Note that in the NaSch model with $v_{\max }=1$ every vehicle moves forward with probability $q=1-p$ in the time step $t+1$ if the site immediately in front of it were empty at the time step $t$; this, is similar to TASEP. But, updating is done in parallel in the NaSch model whereas that in the TASEP is done in a random sequential manner. Nevertheless, the special case of $v_{\max }=1$ for the NaSch model achieves special importance from the fact that so far it has been possible to derive exact analytical results for the NaSch model only in the special limits (a) $p=0$ and arbitrary $v_{\max }$ (which we have already considered), and (b) $v_{\max }=1$ and arbitrary $p$.

\subsubsection{Relation with surface growth models and the phenomenological fluid- dynamical theories of traffic}

The NaSch model with $v_{\max }=1$ can be mapped onto stochastic growth models of one-dimensional surfaces in a two-dimensional medium, the single-step model [135]. Corresponding to each configuration $\left\{\sigma_{j}\right\}$ of the NaSch model in the site-oriented description, one can obtain a unique surface profile $\left\{H_{j}\right\}$ through the relation $H_{j}=\frac{1}{2} \sum_{j \leq k}\left(1-2 \sigma_{k}\right)$ [135,136]. Pictorially one can interpret this mapping as shown in Fig. 16. Particle (hole) movement to the right (left) correspond to local forward growth of the surface via particle deposition. In this scenario a particle evaporation would correspond to a particle (hole) movement to the left (right) which is not allowed in the NaSch model. It is worth pointing out that any quenched disorder in the rate of hopping between two adjacent sites would correspond to columnar quenched disorder in the growth rate for the surface [137].

The surface growth model described above is known to be a discrete counterpart of continuum models of growing surfaces whose dynamics are governed 




Fig. 16. The schematic pictorial representation of the mapping of the NaSch model with $v_{\max }=1$ onto a stochastic model of surface growth.

by the so-called Kardar-Parisi-Zhang equation [135,136]. Since the KardarParisi-Zhang equation can be mapped onto the Burgers equation [53] using the Cole-Hopf transformation [135], it is not surprising that several features of vehicular traffic are described by the NaSch model at the microscopic level and by the noisy Burgers equation for the coarse-grained continuum of the fluid-dynamical theory [138].

\subsection{Limiting cases of the NaSch model}

In spite of the fact that the deterministic limits $p=0$ and $p=1$ of the NaSch model do not capture some of the most essential features of vehicular traffic it may be instructive to examine these limits to gain insight into the features of this simpler scenario. Another limiting case which exhibits a surprisingly complex behaviour is the case $v_{\max }=\infty$.

\subsubsection{NaSch model in the deterministic limit $p=0$}

The NaSch model, a stochastic CA, becomes a deterministic CA in the limit $p=0$. In this special case, the deterministic dynamical update rules of the model can be written as

$$
\begin{aligned}
& v_{n}(t+1)=\min \left[v_{\max }, v_{n}(t)+1, d_{n}(t)-1\right], \\
& x_{n}(t+1)=x_{n}(t)+v_{n}(t+1)
\end{aligned}
$$

which can lead to two types of steady states depending on the density $c$ [113]. At low densities, the system can self-organize so that $d_{n}>v_{\max }$ for all $n$ and, therefore, every vehicle can move with $v_{\max }$, i.e., $v_{n}(t)=v_{\max }$, giving rise to the corresponding flux $c v_{\max }$. This steady-state is, however, possible only if 
enough empty cells are available in front of every vehicle, i.e., for $c \leq c_{m}^{\text {det }}=$ $\left(v_{\max }+1\right)^{-1}$ and the corresponding maximum flux is $J_{\max }^{\text {det }}=v_{\max } /\left(v_{\max }+1\right)$. On the other hand, for $c>c_{m}^{\text {det }}, d_{n}(t)-1 \leq \min \left[v_{n}(t)+1, v_{\max }\right]$ and, therefore, the relevant steady-states are characterized by $v_{n}(t)=d_{n}(t)-1$, i.e., the flow is limited by the density of holes. Since the average distance-headway is $1 / c-1$, the fundamental diagram in the deterministic limit $p=0$ of the NaSch model (for any arbitrary $v_{\max }$ ) is given by the exact expression

$$
J=\min \left(c v_{\max }, 1-c\right) .
$$

Note that the result $v_{n}=1 / c-1$ is identical with Greenshields ansatz $v_{n}=$ $1 / c-1 / c_{\text {jam }}$ if we identify $c_{\text {jam }}=1$.

\subsubsection{NaSch model in the deterministic limit $p=1$}

Aren't the properties of the NaSch model with maximum allowed speed $v_{\max }$, in the deterministic limit $p=1$, exactly identical to those of the same model with maximum allowed speed $v_{\max }-1$ ? Although this expectation may seem to be consistent with the observation that $J=0$ for all $c$ in the special case $v_{\max }=1=p$, the answer to the question posed above is: NO. To understand the subtle features of the deterministic limit $p=1$ one has to consider $v_{\max }>$ 1. You can easily convince yourself that if, for example, $v_{\max }=2$, then, for $c>1 / 3$, all stationary states correspond to $J=0$ because at least one vehicle will have only one empty cell in front (i.e. $d_{n}=2$ ) and it will never succeed in moving forward. For $v_{\max }=2$ and $p=1$, although there are stationary states corresponding to $J \neq 0$ for all $c \leq 1 / 3$, such states are metastable in the sense that any local external perturbation leads to complete breakdown of the flow. If the initial state is random, such metastable states cannot lead to non-zero $J$ because they have a vanishing weight in the thermodynamic limit. Hence, if $p=1$, all random initial states lead to $J=0$ in the stationary state of the NaSch model irrespective of $v_{\max }$ and $c$ !

\subsubsection{NaSch model in the limit $v_{\max }=\infty$}

The limit $v_{\max }=\infty$ has been introduced in [139]. One has to be aware that there are several possible ways of performing this limit since only finite systems of length $L$ can be treated in computer simulations. In [139] the case $v_{\max }=L$ has been investigated $\square$, but other limiting procedures are also possible, e.g. $v_{\max } \propto L^{\alpha}$ with $\alpha>0$ or even $v_{\max }=\infty$ independent of the system size. In principle, these different limiting procedures could lead to different results, but up to now no systematic study has been performed. We therefore restrict ourselves to the case $v_{\max }=L$ studied in [139].

\footnotetext{
${ }^{10}$ See also [113], where the case $p=0$ was studied.
} 
Surprisingly one finds that the fundamental diagram has a form quite different from that of the case of a finite $v_{\max }$. The flow does not vanish in the limit $c \rightarrow$ 0 since already one single car produces a finite value of the flow, $J(c \rightarrow 0)=1$. Due to the hindrance effect of other cars, $J(c)$ is a monotonically decreasing function of the density $c$ (see Fig. 17). Another characteristic feature of the fundamental diagram is the existance of a plateau at flow $J_{P}$ where the value $J_{P}$ depends on the randomization $p$, but not on the system size $L$. The length of the plateau, on the other hand, increases with $L$.



Fig. 17. Fundamental diagram of the NaSch model with $v_{\max }=L$ for a system size of $L=1000$ (from [139]).

What is the microscopic structure of the stationary state leading to such a fundamental diagram? At low densities, where flow $J$ is larger than the plateau value $J_{P}$, the cars tend to be uniformly distributed just as in the deterministic case $p=0$ (see Sec. 8.2.1). For densities in the plateau regime, however, one jam exists in the system, whereas for higher densities there is more than one jam. In the thermodynamic limit, one expects a phase transition at $c=0$ between a jamless phase with $J=1$ and a phase with one jam and flow $J_{P}$ [139]. Increasing the density further, more jams develop and the plateau ceases. Note that this behaviour is completely different from the prediction of mean-field theory in that limit [140] (see Sec. 8.3.1) showing the importance of correlations.

\subsection{Analytical theories of the NaSch model with periodic boundary conditions}

CA are, by design, ideal for large scale computer simulations. However, proper interpretations of the numerical data obtained from computer simulations are not always quite straightforward because of the finite-size effects and "numerical noise". One cannot deny the importance of exact analytical results in providing a testing ground for the computer codes. On the other hand, the parallel updating makes exact analytical solution of CA models very difficult. Nevertheless, even in those situations where exact solutions are not possible, 
a combination of approximate analytical treatments and computer simulation often turns out out to be very powerful method of analysis of a problem. This approach has been quite successful in recent years in the studies of the NaSch model and its generalizations.

Before we proceed with the analytical theories in the non-deterministic NaSch model, we would like to point out that the fundamental diagram $J(c)$ is known exactly for arbitrary $v_{\max }$ and $p$ in the two limits $c \rightarrow 0$ and $c \rightarrow 1$. In the former case, $J \simeq c v_{F}$ where $v_{F}=v_{\max }-p$ is the free-flow velocity. On the other hand, in the latter case, $J \simeq(1-p)(1-c)$ as flow is determined by holes moving backwards at a speed $1-p$.

\subsubsection{Site-oriented naive mean-field theory for the NaSch model}

In the "site-oriented" theories one describes the state of the finite system of length $L$ by completely specifying the state of each site, i.e., by the set $\left(\sigma_{1}, \sigma_{2}, \ldots, \sigma_{L}\right)$ where $\sigma_{j}(j=1,2, \ldots, L)$ can, in principle, take $v_{\max }+2$ values one of which represents an empty site while the remaining $v_{\max }+1$ correspond to the $v_{\max }+1$ possible values of the speed of the vehicle occupying the site $j$. In some of the analytical calculations of steady-state properties of the NaSch model one follows, for convenience, the sequence $2-3-4-1$, instead of $1-2-3-4$ of the stages of updating [140] as this merely shifts the starting step and, therefore, does not influence the steady-state properties of the model. The advantage of this new sequence is that, in a site-oriented theory, the variable $\sigma_{j}$ can now take $v_{\max }+1$ values as none of the vehicles can have a speed $v=0$ at the end of the acceleration stage of the updating.

Let us introduce the lattice gas variables $n(i ; t)$ through the following definition: $n(i ; t)=0$ if the site labeled by $i$ is empty and $n(i ; t)=1$ if it is occupied by a vehicle (irrespective of the speed). Obviously, the space-average of $n(i ; t)$ is the density of the vehicles, i.e., $\sum_{i} n(i ; t) / L=c$. Suppose, $c_{v}(i ; t)$ is the probability that there is a vehicle with speed $v\left(v=0,1,2, \ldots, v_{\max }\right)$ at the site $i$ at the time step $t$. Obviously, $c(i ; t)=\sum_{v=0}^{v_{\max }} c_{v}(i ; t)$ is the probability that the site $i$ is occupied by a vehicle at the time step $t$ and $d(i ; t)=1-c(i ; t)$ is the corresponding probability that the site $i$ is empty at the time step $t$.

In the naive site-oriented mean-field (SOMF) approximation for the NaSch model one writes down the equations relating $c_{v}(i ; t+1)\left(v=1, \ldots, v_{\max }\right)$ with the corresponding probabilities at time $t$ and, then, solves the equations in the steady-state (see the Appendix $\mathrm{C}$ for a detailed derivation of these equations for arbitrary $\left.v_{\max }\right)$. In the simplest case of $v_{\max }=1$ and periodic boundary conditions one gets [140] 


$$
\begin{aligned}
& c_{0}(i ; t+1)=c(i ; t) c(i+1 ; t)+p c(i ; t) d(i+1 ; t), \\
& c_{1}(i ; t+1)=q c(i-1 ; t) d(i ; t) .
\end{aligned}
$$

The equation (64) expresses the simple fact that at the time step $t+1$ the speed of the vehicle at the $i$-th site can be zero either because the site $i+1$ was occupied at time $t$ or because of random deceleration (if the site $i+1$ was empty at time $t$ ). Similarly, the equation (65) implies that the speed of the vehicle at the site $i$ can be 1 at time $t+1$ if at the time step $t$ the site $i$ was empty while the site $i-1$ was occupied by a vehicle which did not decelerate during the random deceleration stage of updating.

In the steady state, $c_{v}(i, t)$ are independent of $t$. Besides, if periodic boundary conditions are imposed, the $i$-dependence of $c_{v}(i)$ also drops out in the translational-invariant steady-state. Therefore, in the steady-state

$$
J=c_{1}=q c(1-c)
$$

It turns out [140] that the naive SOMF underestimates the flux for all $v_{\max }$. Curiously, if instead of parallel updating one uses the random sequential updating, the NaSch model with $v_{\max }=1$ reduces to the TASEP for which the equation (66) is known to be the exact expression for the corresponding flux (see, e.g., $[20])$ !

\subsubsection{Paradisical mean-field theory of the NaSch model}

What are the reasons for these differences arising from parallel updating and random sequential updating? There are "Garden of Eden" (GoE) [141] states (dynamically forbidden states) [142] of the NaSch model which cannot be reached by the parallel updating whereas no state is dynamically forbidden if the updating is done in a random sequential manner. For example, the configuration shown in Fig. 18 is a GoE state $\square$ because it could occur at time $t$ only if the two vehicles occupied the same cell simultaneously at time $t-1$.

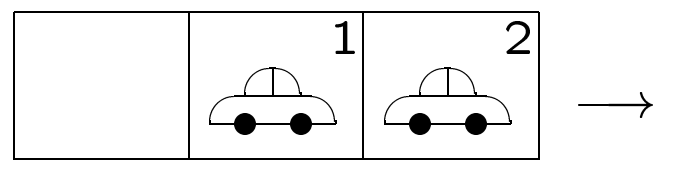

Fig. 18. A GoE state for the NaSch model with $v_{\max } \geq 2$.

The naive SOMF theory, discussed in the preceding subsection, does not exclude the GoE states. On the other hand, results of the paradisical mean-field

$\overline{11}$ The configuration shown in Fig. 12 is also a GoE state! 
(pMF) theory are derived by repeating the calculations of the naive SOMF theory excluding all the GoE states from consideration. The exact expression, given in the next subsection, for the flux in the steady-state of the NaSch model with $v_{\max }=1$ is obtained in the pMF theory (see Appendix D for detailed calculations), thereby indicating that the only source of correlation in this case is the parallel updating [142]. But, for $v_{\max }>1$, there are other sources of correlation because of which exclusion of the GoE states merely improves the naive SOMF estimate of the flux (Fig. 19) but does not yield exact results $[142,143]$.
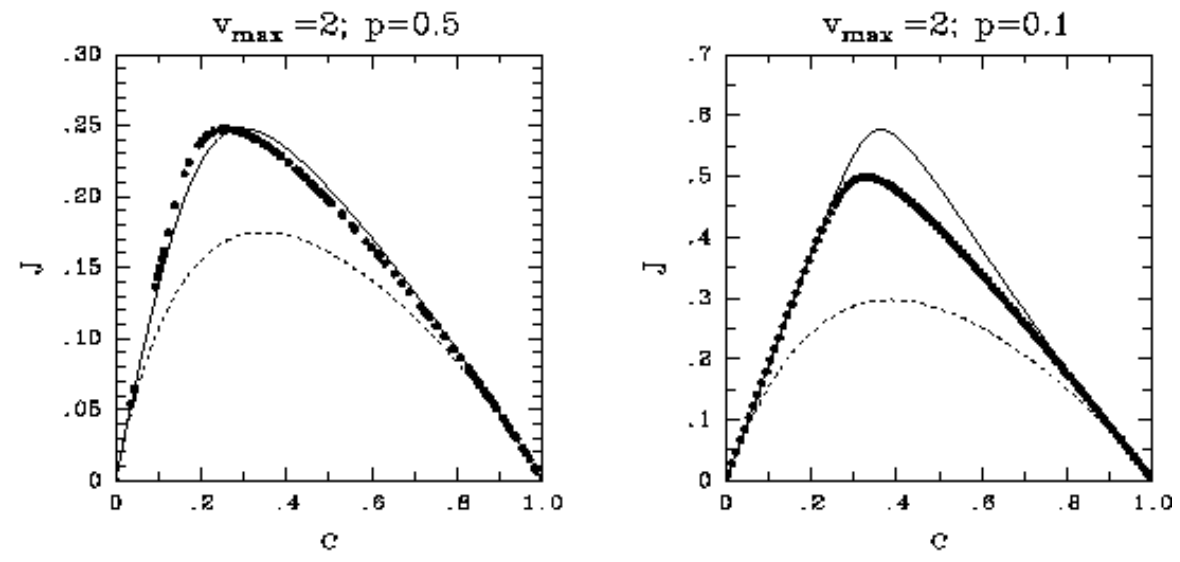

Fig. 19. Fundamental diagram for $v_{\max }=2$ and $p=0.5$ (left) and $p=0.1$ (right). Comparison of paradisical MFT (full line) with results from computer simulations $(\bullet)$ and the naive MFT (dotted line).

\subsubsection{Site-oriented cluster-theoretic approach to the NaSch Model}

The site-oriented cluster theoretic approach leads to a systematic improvement of the naive SOMF theory of the NaSch model. We define a $n$-cluster to be a collection of $n$ successive sites and denote the probability of finding an $n$ cluster in the state $\left(\sigma_{1}, \sigma_{2}, \ldots, \sigma_{n}\right)$ in the steady-state of the system by the symbol $P_{n}\left(\sigma_{1}, \sigma_{2}, \ldots, \sigma_{n}\right)$. In the general $n$-cluster approximation [140], one divides the lattice into "clusters" of length $n$ such that two neighbouring clusters have $n-1$ sites in common (see Fig. 20); an $n$-cluster is treated exactly and the cluster is coupled to the rest of the system in a self-consistent way, as we shall show in this subsection. Even without any calculation, one would expect that, for a given $v_{\max }$, the $n$-cluster approximation should yield more accurate results with increasing $n$ and should give exact results in the limit $n \rightarrow \infty$. Fortunately, in the special case $v_{\max }=1$ exact results are obtained already for $n$ as small as 2, i.e., the results of 2-cluster calculations are exact for $v_{\max }=1$ [140].

Let us first explain the key concepts involved in the cluster theory. It is straightforward to verify, for example, in the special case of $v_{\max }=1$, that the state of the 2 -cluster $\sigma_{i}, \sigma_{i+1}$ at time $t+1$ depends on the state of the 4 -cluster 
a)

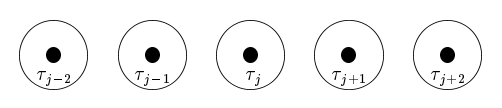

b)

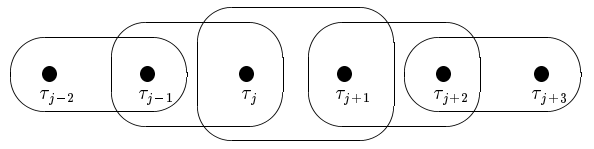

c)

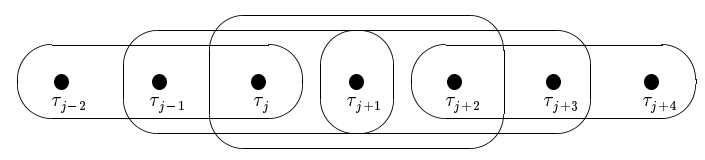

Fig. 20. Decomposition of a lattice into (a) 1-clusters (b) 2-clusters and (c) 3-clusters in the SOMF theory.

$\left(\tau_{i-1}, \tau_{i}, \tau_{i+1}, \tau_{i+2}\right)$ at time $t$. In general, in the $n$-cluster approximation for an arbitrary $v_{\max }$ one has to take into account the vehicles that can enter an $n$-cluster from one of the $v_{\max }$ cells to its left and can leave it to occupy one of the $v_{\max }$ cells to its right. Therefore, in general, the state $\sigma_{j}, \sigma_{j+1}, \ldots \sigma_{j+n-1}$ of an $n$-cluster at time $t+1$ depends on the state of a $n+2 v_{\max }$ cluster $\tau_{j-v_{\max }}, \ldots \tau_{j}, \tau_{j+1}, \ldots, \tau_{j+n-1}, \ldots, \tau_{j+n-1+v_{\max }}$ at time $t$. Therefore, in the special case of $v_{\max }=1$, the master equations

$$
P_{2}\left(\sigma_{i}, \sigma_{i+1} ; t+1\right)=\sum_{\tau_{j}} W\left(\sigma_{i}, \sigma_{i+1} \mid \tau_{i-1}, \tau_{i}, \tau_{i+1}, \tau_{i+2}\right) P_{4}\left(\tau_{i-1}, \tau_{i}, \tau_{i+1}, \tau_{i+2} ; t\right)
$$

governing the time evolution of the 2-cluster probabilities $P_{2}\left(\sigma_{i}, \sigma_{i+1}\right)$ involve the 4 -cluster probabilities for all those configurations $\left(\tau_{i-1}, \tau_{i}, \tau_{i+1}, \tau_{i+2} ; t\right)$ which can lead to the 2-cluster configuration $\left(\sigma_{i}, \sigma_{i+1} ; t+1\right)$ under consideration as well as the corresponding transition probabilities $W\left(\sigma_{i}, \sigma_{i+1} \mid \tau_{i-1}, \tau_{i}, \tau_{i+1}, \tau_{i+2}\right)$. Similarly, the master equation governing the time evolution of the 4-cluster probabilities $P_{4}\left(\tau_{i-1}, \tau_{i}, \tau_{i+1}, \tau_{i+2}\right)$ involve 6 -cluster probabilities, and so on. In order to obtain a closed set of equations one has to truncate this hierarchy in an appropriate manner; in the $n$-cluster approximation one expresses the $\left(n+2 v_{\max }\right)$-cluster probabilities in terms of products of $n$-cluster probabilities.

The $n$-cluster approximation represented geometrically in Fig. 20 for $n=1$ 
can be expressed mathematically as

$$
P\left(\tau_{j-2}, \tau_{j-1}, \tau_{j}, \tau_{j+1}, \tau_{j+2}\right)=\prod_{i=\tau_{j-2}}^{\tau_{j+2}} P_{1}\left(\tau_{i}\right)
$$

Thus, 1-cluster approximation is equivalent to the naive SOMF approximation. The 2-cluster approximation represented geometrically in Fig. 20 can be expressed mathematically as [140]

$$
\begin{aligned}
& P\left(\tau_{j-2}, \tau_{j-1}, \tau_{j}, \tau_{j+1}, \tau_{j+2}, \tau_{j+3}\right) \propto P_{2}\left(\tau_{j-2}, \tau_{j-1}\right) P_{2}\left(\tau_{j-1}, \tau_{j}\right) P_{2}\left(\tau_{j}, \tau_{j+1}\right) \\
& \cdot P_{2}\left(\tau_{j+1}, \tau_{j+2}\right) P_{2}\left(\tau_{j+2}, \tau_{j+3}\right)
\end{aligned}
$$

or, more precisely,

$$
\begin{aligned}
P\left(\tau_{j-2}, \tau_{j-1}, \tau_{j}, \tau_{j+1}, \tau_{j+2}, \tau_{j+3}\right)= & P_{2}\left(\tau_{j-2} \mid \underline{\tau_{j-1}}\right) P_{2}\left(\tau_{j-1} \mid \underline{\tau_{j}}\right) P_{2}\left(\tau_{j}, \tau_{j+1}\right) \\
& \cdot P_{2}\left(\underline{\tau_{j+1} \mid \tau_{j+2}}\right) P_{2}\left(\underline{\tau_{j+2} \mid} \mid \tau_{j+3}\right)
\end{aligned}
$$

where

$$
P_{2}\left(\tau_{j-1} \mid \underline{\tau_{j}}\right)=\frac{P_{2}\left(\tau_{j-1}, \tau_{j}\right)}{\sum_{\tau_{j-1}} P_{2}\left(\tau_{j-1}, \tau_{j}\right)}
$$

are 2-cluster conditional probabilities. Similarly, the 3-cluster approximation consists of the approximate factorization

$$
\begin{aligned}
& P\left(\tau_{j-2}, \tau_{j-1}, \tau_{j}, \tau_{j+1}, \tau_{j+2}, \tau_{j+3}, \tau_{j+4}\right)=P_{3}\left(\tau_{j-2} \mid \underline{\tau_{j-1}, \tau_{j}}\right) P_{3}\left(\tau_{j-1} \mid \underline{\tau_{j}, \tau_{j+1}}\right) \\
& \cdot P_{3}\left(\tau_{j}, \tau_{j+1}, \tau_{j+2}\right) P_{3}\left(\underline{\tau_{j+1}, \tau_{j+2}} \mid \tau_{j+3}\right) P_{3}\left(\underline{\tau_{j+2}, \tau_{j+3}} \mid \tau_{j+4}\right) .
\end{aligned}
$$

Analoguous factorizations hold for an arbitrary number of sites on the lefthand-side of (68),(70) and (72).

Let us now illustrate the scheme of the cluster calculations for the NaSch model by carrying out the calculation for the simplest case, namely, the 2cluster calculations for $v_{\max }=1$. For convenience, one follows the sequence $2-3-4-1$, instead of $1-2-3-4$ of the stages of updating so that, for $v_{\max }=1$, a two-state variable $\sigma$ is adequate to describe the state of a lattice site; $\sigma=0,1$ correspond, respectively, to an empty site and a site occupied by a vehicle with speed 1. Thus, in the special case of $v_{\max }=1$ one would need only four 2cluster probabilities, namely, $P_{2}(0,0), P_{2}(1,0), P_{2}(0,1), P_{2}(1,1)$. Interestingly, the constraints

$$
P_{2}(1,0)+P_{2}(1,1)=c
$$


and

$$
P_{2}(0,0)+P_{2}(0,1)=1-c
$$

together with the particle-hole symmetry

$$
P_{2}(1,0)=P_{2}(0,1)
$$

leave only one of the four 2-cluster probabilities, say, $P_{2}(1,0)$, as an independent variable which one needs to calculate by solving the corresponding master equation. For general $v_{\max }$, the $n$-cluster approximation on the right hand side of the master equation leads to $\left(v_{\max }+1\right)^{n}$ nonlinear equations; the number of independent equations gets reduced by the so-called Kolmogorov consistency conditions [144].

Using (70) one factorizes the 4-cluster probabilities on the right-hand-side of (67) for $P_{2}(1,0)$ in terms of 2-cluster conditional probabilities. In the first column of the table in Fig. 21 we list all those configurations $\left(\tau_{i-1}, \tau_{i}, \tau_{i+1}, \tau_{i+2} ; t\right)$ which can lead to the configurations, shown in the second column, which is the exhaustive list of the 4-cluster configurations each having $\sigma_{i}=1, \sigma_{i+1}=0$; the corresponding transition probabilities $W\left(1,0 \mid \tau_{i-1}, \tau_{i}, \tau_{i+1}, \tau_{i+2}\right)$ are given in the third column.

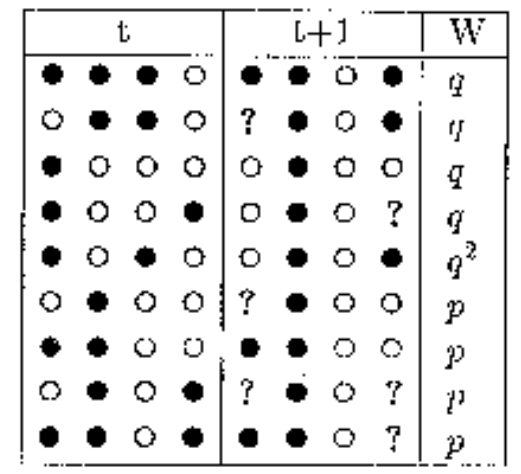

Fig. 21. The four circles in the first two columns represent the states of the sites of a 4-cluster; the open and filled circles correspond, respectively, to empty sites and sites occupied by vehicles with speed $v=1$.

Using the configurations at $t$ and $t+1$ as well as the corresponding transition probabilities given in table in Fig. 21 the master equation $(67)$ for $P_{2}(1,0)$ reduces to the quadratic algebraic equation

$$
q y^{2}-y+c(1-c)=0
$$

where we have used the shorthand notation $y=P_{2}(1,0)$. Solving this quadratic equation we get [140] (see also [145])

$$
P_{2}(1,0)=\frac{1}{2 q}[1-\sqrt{1-4 q c(1-c)}]
$$


and, hence, $P_{2}(1,1), P_{2}(0,0), P_{2}(0,1)$ from the equations $(73),(74)$ and $(75)$. Moreover, the expression (77) establishes that $P_{2}(1,0) \geq P_{1}(1) P_{1}(0)=c(1-c)$, which indicates an effective particle-hole attraction (particle-particle repulsion) in the NaSch model with $v_{\max }=1$. Furthermore, from equation (77) one gets the expression

$$
J(c, p)=q P_{2}(1,0)=\frac{1}{2}[1-\sqrt{1-4 q c(1-c)}]
$$

which can be proved [140] to be the exact expression for the corresponding flux. It is not difficult to carry out 2-cluster calculations for higher values of $v_{\max }$, but one gets only approximate results for $v_{\max }>1$ [140].
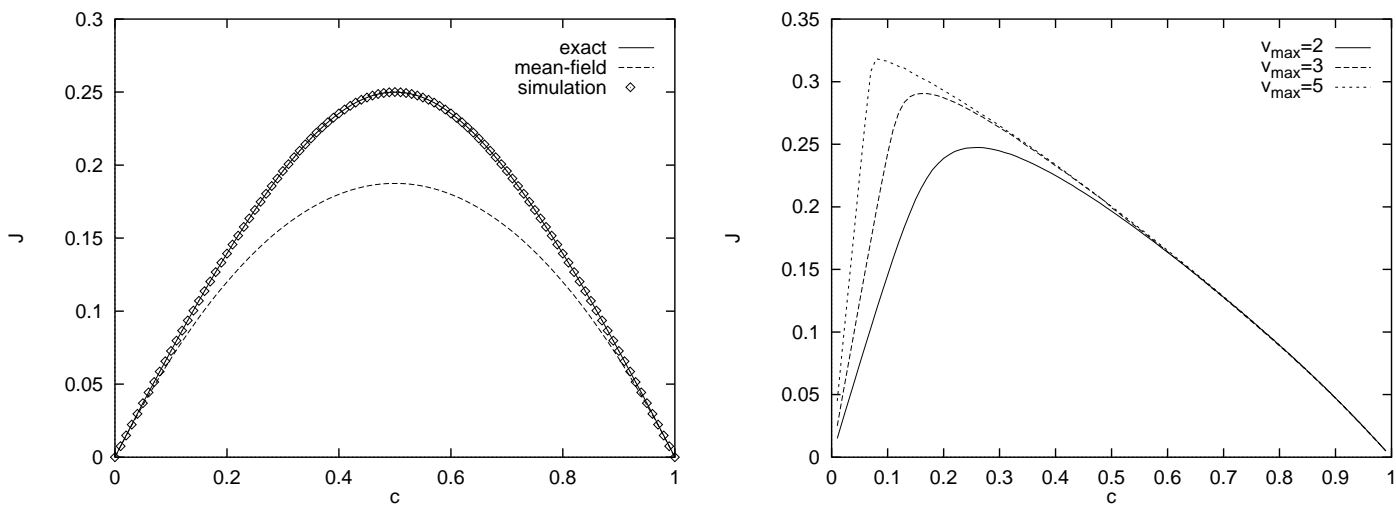

Fig. 22. The fundamental diagram in the NaSch model for (a) $v_{\max }=1$ and (b) $v_{\max }>1$, both for $p=0.25$. The data for all $v_{\max }>1$ have been obtained through computer simulations.

An interesting feature of the expression (78) is that the flux is invariant under charge conjugation, i.e., under the operation $c \rightarrow(1-c)$ which interchanges particles and holes. Therefore, the fundamental diagram is symmetric about $c=1 / 2$ when $v_{\max }=1$ (see Fig. 22(a)). Although this symmetry breaks down for all $v_{\max }>1$ (see Fig. 22(b)), the corresponding fundamental diagrams appear more realistic. Moreover, for given $p$, the magnitude of $c_{m}$ decreases with increasing $v_{\max }$ as the higher is the $v_{\max }$ the longer is the effective range of interaction of the vehicles (see Fig. 22). Furthermore, for $v_{\max }=1$, flux merely decreases with increasing $p$ (see eqn. (78)), but remains symmetric about $c=1 / 2=c_{m}$. On the other hand, for all $v_{\max }>1$, increasing $p$ not only leads to smaller flux but also lowers $c_{m}$ (Fig. 23).

For $v_{\max }>1$, one needs to carry out higher order cluster calculations $[140,143]$ to get more accurate results than those obtained in the 2-cluster approximation. For $v_{\max }=2$, the fundamental diagrams obtained from the $n$-cluster approximation $(n=1,2, . ., 5)$ are compared in Fig. 24 with the Monte Carlo data. This comparison clearly establishes a rapid convergence with increasing $n$ and already for $n=4$ the difference between the cluster calculation and MC data is extremely small. In [143] the cluster probabilities for $v_{\max }=2$ 


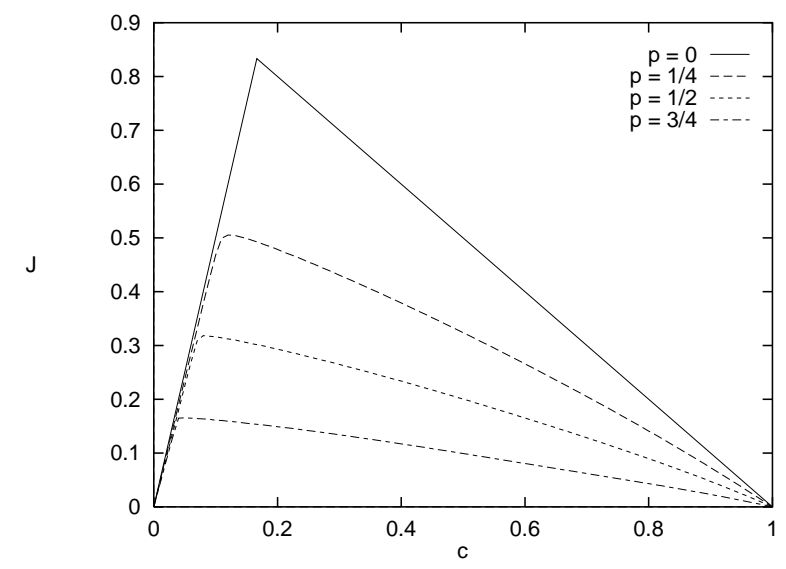

Fig. 23. The fundamental diagrams in the NaSch model with $v_{\max }=5$ are plotted for a few values of $p$.

have been obtained from computer simulations. The results suggest that the $n$-cluster approximation for $n \geq 3$ becomes asymptotically exact in the limit $p \rightarrow 0$.

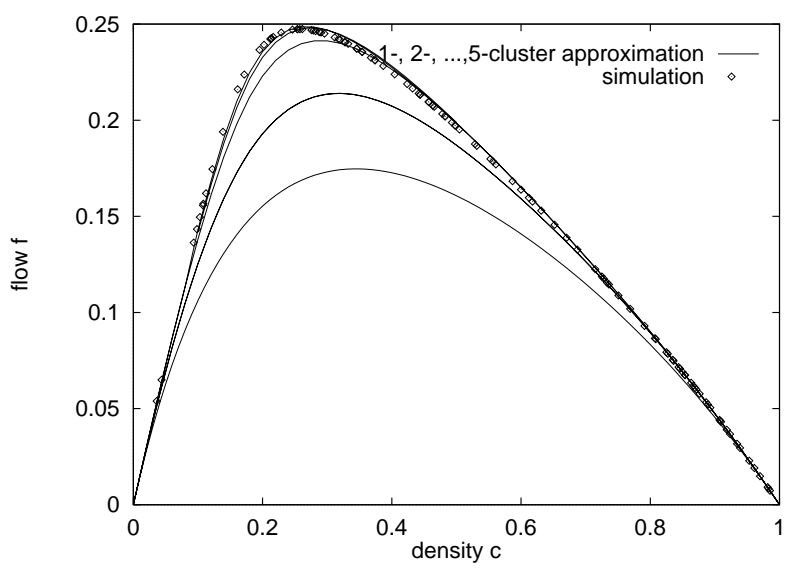

Fig. 24. The fundamental diagrams in the NaSch model with $v_{\max }=2$ in the $n$-cluster approximation $(n=1,2, . ., 5)$.

\subsubsection{Car-oriented mean-field theory of the NaSch model}

In the "car-oriented" theories the state of the traffic system is described by specifying the positions and speeds of all the $N$ vehicles in the system [146]. Suppose, $P_{n}(t)$ is the probability to find at time $t$ exactly $n$ empty sites immediately in front of a vehicle. Another auxiliary quantity, which turns out to be very convenient to use in several different calculations, is $g(t)$, the probability at time $t$ that a vehicle moves in the next time step. These two sets of quantities, namely, $P_{n}(t)$ and $g(t)$ are related. For example, in the NaSch model with $v_{\max }=1$, a vehicle will move in the next time step if there is at least one empty cell in front of it (probability $\sum_{n \geq 1} P_{n}(t)$ ) and 
if it does not decelerate in the randomization step (probability $q$ ); therefore, $g(t)=q\left[\sum_{n \geq 1} P_{n}(t)\right]=q\left[1-P_{0}(t)\right]$. Hence the flux $J(c, p)$ can be obtained from $J(c, p)=c g=c q\left[1-P_{0}\right]$.

The essence of the car-oriented mean-field (COMF) approximation [146] is to neglect the correlations between the gaps in front of the successive cars ${ }^{12}$. The equations describing the time evolution of the probabilities $P_{n}(t)$, under this approximation (see Appendix $\mathrm{E}$ for the derivation of these equations), can be solved in the steady-state using a generating function technique [146]. Following this approach, in the special case $v_{\max }=1$, one recovers the exact expression (78) for $J(c, p)=c q\left[1-P_{0}\right]$.

For $v_{\max }=2$ one has to distinguish between $P_{n}(v=1)$ and $P_{n}(v=2)$. Moreover, one has to generalize the quantity $g$ to $g_{\alpha}$, the probability that the vehicle moves $\alpha$ cells $(\alpha=1,2)$ in the next time step. Applying the same generating function techniques as for $v_{\max }=1$, one can also solve the coupled sets of steady-state equations for $P_{n}(v=1)$ and $P_{n}(v=2)$ for $v_{\max }=2$ but gets only approximate results [146].

Interestingly, finite size of the system affects the equations for $v_{\max }=2 \mathrm{in}$ a much more dramatic way [143] than those for $v_{\max }=1$ thereby revealing the intrinsic qualitative differences in the nature of correlations in the NaSch model for $v_{\max }=1$ and $v_{\max }>1$.

Comparisons with Monte Carlo simulations show that in contrast to the 3cluster approximation for $v_{\max }=2$ COMF does not become asymptotically exact in the limit $p \rightarrow 0$. This implies that even in this limit correlations between the headways are not negligible. It is interesting, however, that for the fundamental diagram one finds an excellent agreement between MC simulations and the predictions of COMF [146] for $p \rightarrow 0$. The reason is that in the deterministic limit many configurations exist which produce the same flow. COMF is not able to identify the dominating structures correctly, but nevertheless can predict the correct current.

\subsubsection{Microscopic structure of the stationary state}

As we have seen MFT underestimates the flow in the stationary state considerably. Deviations become larger for higher velocities $v_{\max }$. This shows the importance of correlations. As described above a particle-hole attraction exist. Using the 2-cluster probabilities for $v_{\max }=1$ this attraction can be expressed in mathematical form as $P_{2}(1,0)>P_{1}(1) P_{1}(0)=c(1-c)$.

$\overline{12}$ A similar approach, the so-called interparticle distribution functions technique, is used for studying reaction-diffusion systems [147]. 
For $v_{\max }=1$ all improvements of MFT (2-Cluster, COMF and pMFT) are exact. Here only correlations between neighbouring cells are important. The fact that pMFT is exact shows that no 'true' correlations exist. All correlations have their origin in the existence of GoE states. This also helps to understand why for random-sequential dynamics already MFT is exact for $v_{\max }=1$ and the stationary state is uncorrelated. The reason is simply that for randomsequential dynamics no GoE states exist!

The situation changes for higher velocities $v_{\max }>1$. Here pMFT is no longer exact ${ }^{13}$. Therefore 'true' correlations exist. This corresponds to the observation made in [140] that the NaSch model shows a qualitatively different behaviour for $v_{\max }=1$ and $v_{\max }>1$. Furthermore it explains why so far the exact determination of the stationary state for $v_{\max }>1$ has not been possible.

It is interesting to investigate how the microscopic structure of the stationary state depends on the randomization $p$. For $p=0$ we have seen in Sec. 8.2.1 that for densities $c \leq 1 /\left(v_{\max }+1\right)$ the vehicles arrange themselves in such a way that all headways are at least $v_{\max }$. This is no longer possible for larger densities, but still the vehicles have the tendency to maximize their headway. Furthermore, for $p=0$ no spontaneous formation of jams exists since overreactions are not possible. The behaviour in this limit can be interpreted as coming from a kind of "repulsive interaction" between the vehicles.

The behaviour for $p=1$ is a little bit different. Here we have seen in Sec. 8.2.2 that metastable states with finite flow exist for $c \leq 1 / 3$ and $v_{\max }>1$.

For $0<p<1$ the microscopic structure interpolates between these two limiting cases. This can be seen by analysing the 3-cluster probabilities obtained from Monte Carlo simulations [143]. For small $p$ the microscopic structure of the stationary state is determined by the 'repulsive interactions' between vehicles. With increasing $p$ one finds a tendency towards phase separation into jammed and free-flow regions. A standing vehicle is able to induce a jam even at low densities since the restart probability is small. The jams formed are typically not compact, but of the form '.0.0.0.' since a vehicle approaching the jam slows down in the randomization step with a rather high probability.

Concluding one might say that the microscopic structure for $0<p<1$ is determined by the competition of the two "fixed points" $p=0$ and $p=1$.

\footnotetext{
$\overline{13}$ Note that for random-sequential dynamics also MFT is no longer exact!
} 


\subsection{Spatio-temporal organization of vehicles; is there a phase transition?}

The density $c_{m}$ corresponding to maximum flux is an obvious first candidate for a critical density separating the regimes of free-flow and congested flow in the NaSch model. We shall show in this subsection that this, indeed, is a critical point provided $p=0$. However, in spite of strong indications that, probably, a noise-induced smearing of the transition takes place when $p \neq 0$, rigorous proofs are still lacking.

\section{- Order parameter}

For a proper description of a phase transition one should introduce an appropriate order parameter which can distinguish the two phases because of its different qualitative behavior within the two phases [24,25].

A first candidate [148] for the NaSch model would be the average fraction of vehicles at rest, i.e., with instantaneous speed $v=0$. In the deterministic limit $p=0$ this, indeed, serves the purpose of the order parameter for the sharp transition at $c_{m}^{d e t}$ from the free-flowing dynamical phase to the congested dynamical phase. But, in the general case of non-zero $p$, there is a non-vanishing probability that a vehicle comes to an instantaneous rest merely because of random braking even at extremely low density $c$; this probability is $p$ for $v_{\max }=1$ and decreases with increasing $c$.

The next obvious choice would be [149] the density of nearest-neighbor pairs in the stationary state

$$
m=\frac{1}{T} \frac{1}{L} \sum_{t=1}^{T} \sum_{j=1}^{L} n_{j} n_{j+1},
$$

where, as defined earlier, $n_{j}=0$ for an empty cell and $n_{j}=1$ for a cell occupied by a vehicle (irrespective of its velocity). Because of the step 2 of the updating rule (deceleration due to other vehicles) $m$ gives the space-timeaveraged density of those vehicles with velocity 0 which had to brake due to the next vehicle ahead.

The Fig. 25(a) shows that $m$ vanishes at $c_{m}^{\text {det }}$ if $p=0$. On the other hand if $p \neq 0, m$ does not vanish even for $c<c_{m}$ although $m$ becomes rather small at small densities (see Fig. 25(b)).

We now present a heuristic argument to point out why any quantity related to the fraction of jammed vehicles is non-zero at any density $c>0$ and, hence, inadequate to serve as an order parameter [150]. To slow down to $v_{\max }-2$ a vehicle must be hindered by one randomly braking vehicle in front. Similarly, to reach a speed $v_{\max }-3$ a vehicle must find two vehicles within the range of 

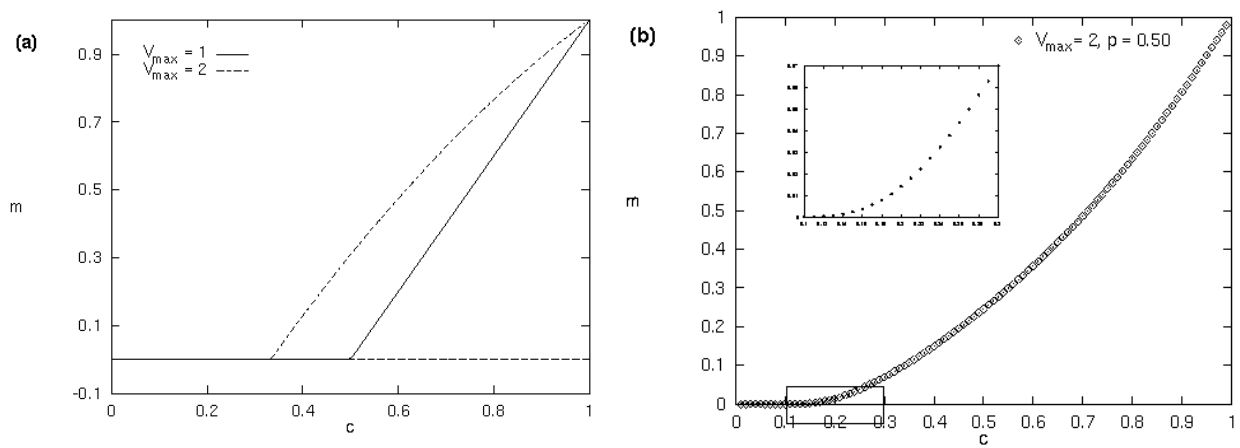

Fig. 25. The density-dependence of the order parameter $m$ in (a) the deterministic limit $p=0$ of the NaSch model $\left(v_{\max }=1,2\right)$ and $(\mathrm{b})$ the NaSch model with $v_{\max }=2, p=0.5$.

interaction, and so on. The probability for $n$ vehicles to be found in the close vicinity of a given vehicle is proportional to $c^{n}$. Therefore, the probability $P_{v}(c)$ of finding a vehicle with speed $v<v_{\max }-1$ is proportional to $c^{v_{\max }-1-v}$ and, hence, even for $v=0, P_{v}(c)$ is, in general, non-zero for all $c \rightarrow 0$.

- Spatial correlations

A striking feature of second-order phase transitions is the occurrence of a diverging length scale at criticality and a corresponding algebraic decay of the correlation function $[24,25]$. Using lattice gas variables $n_{j}$, the equal-time density-density correlation function is defined by

$$
G(r)=\frac{1}{T} \frac{1}{L} \sum_{t=1}^{T} \sum_{j=1}^{L} n_{j} n_{j+r}-c^{2} .
$$

which measures the correlations in the density fluctuations that occur at the same time at two different points in space separated by a distance $r$.

Again it is very instructive to consider first the deterministic case $p=0$ (Fig. 26(a)). Since, as argued before, there are exactly $v_{\max }$ empty sites in front of each vehicle at $c=c_{m}^{\text {det }}$ the correlation function at $c=c_{m}^{\text {det }}$ is given by

$$
G(r)= \begin{cases}c_{m}^{\operatorname{det}}\left(1-c_{m}^{\text {det }}\right) & \text { for } r \equiv 0 \bmod \left(v_{\max }+1\right) \\ -\left(c_{m}^{\text {det }}\right)^{2} & \text { else. }\end{cases}
$$

For all $v_{\max }$ the correlation function for small non-zero $p$ (Fig. 26(b)) has essentially the same structure as that for $p=0$ (Fig. 26(a)) but the amplitude decays exponentially [149] for all $c$. In the general case of non-vanishing $p$, the asymptotic behaviour $(r \rightarrow \infty)$ of the correlation length $\xi$ can be obtained analytically [143] only for $v_{\max }=1$. It turns out that, for given $p, \xi$ is maximum at $c=1 / 2=c_{m}$ and that $\xi(c=1 / 2) \propto p^{-1 / 2}$. Thus, for $v_{\max }=1, \xi$ diverges only for $p=0$ but remains finite for all non-zero $p$. For $v_{\max }>1$ the 

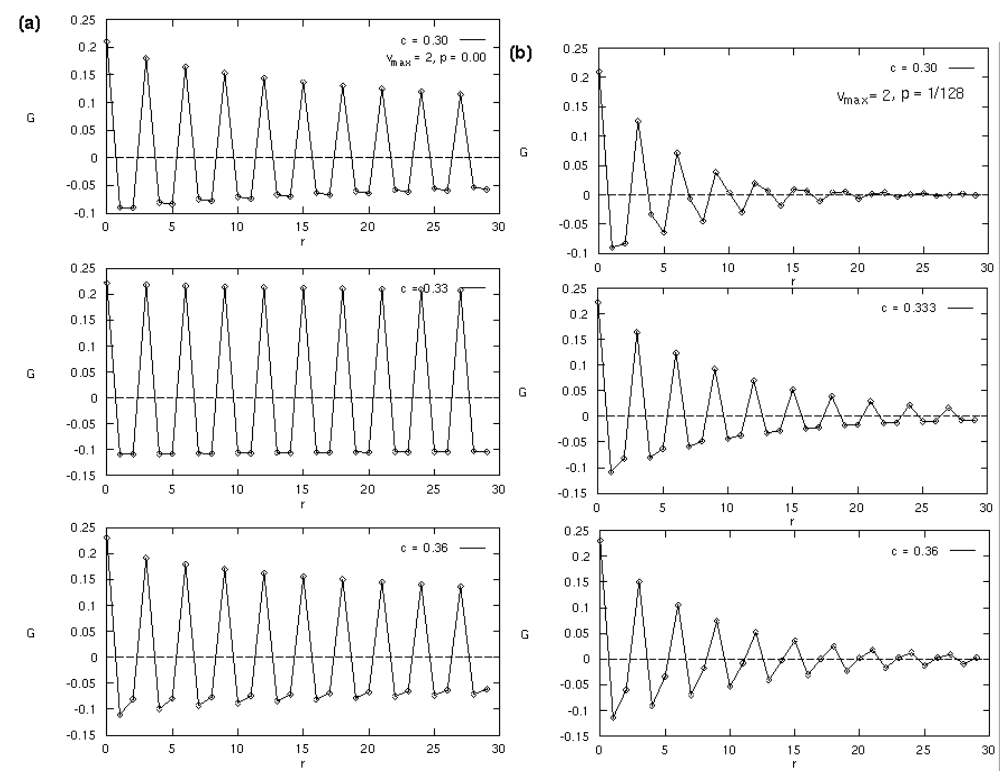

Fig. 26. The density-density correlation function $G(r)$ (a) the deterministic limit $p=0$ of the NaSch model $\left(v_{\max }=2\right)$ and $(\mathrm{b})$ the NaSch model with $v_{\max }=2$, $p=1 / 128$.

trend of variation of $\xi$ with $c$ (Fig. 27(a)) in the vicinity of $c_{m}$ is the same as that for $v_{\max }=1$ [149]. Moreover, for $v_{\max }>1$, the maximum value of the correlation length, $\xi_{\max }$ plotted against $p$ (Fig. 27(b)), is also consistent with the corresponding trend of variation for $v_{\max }=1$. Thus, the correlation function $G(r)$ gives a strong indication that the NaSch model exhibits a second order phase transition, at $c=c_{m}^{d e t}$, only for $p=0$ but this transition is smeared out if $p \neq 0$. This noise-induced smearing of the phase transition in the NaSch model is very similar to the smearing of critical phenomena by finite-size effects.
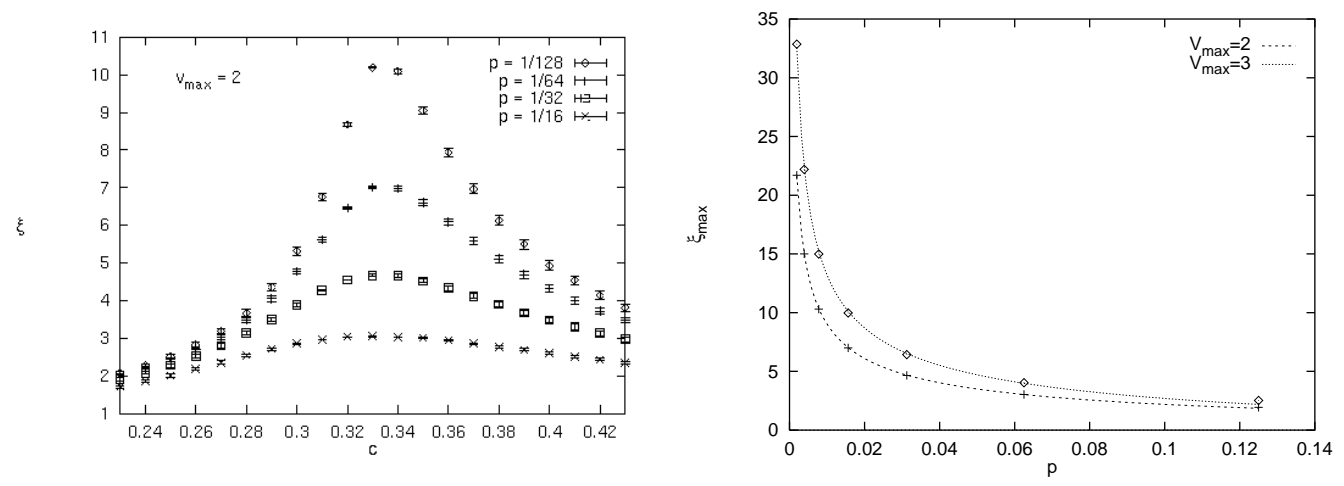

Fig. 27. The dependence of (a) $\xi(c, p)$ on $c$ for four different values of $p\left(v_{\max }=2\right)$ and (b) $\xi_{\max }$ on $p\left(v_{\max }=2,3\right)$.

- Distribution of lifetimes of jams

Another quantity which should be able to give information about the nature 
of the transition from free-flow to the jammed regime is the distribution of lifetimes of jams. Following Nagel [151] each vehicle which has a velocity less than $v_{\max }$ before the randomization step will be considered jammed. This definition is motivated by the cruise-control limit (see Sec. 9.1.1) where it is more natural than in the NaSch model. One expects, however, that the long-time behaviour of the lifetime distribution is independent of the exact definition of a jam. The short-time behaviour, on the other hand, might differ strongly, e.g. for "compact jams" where a jam is defined as a series of consecutive standing vehicles without any empty cells in between.

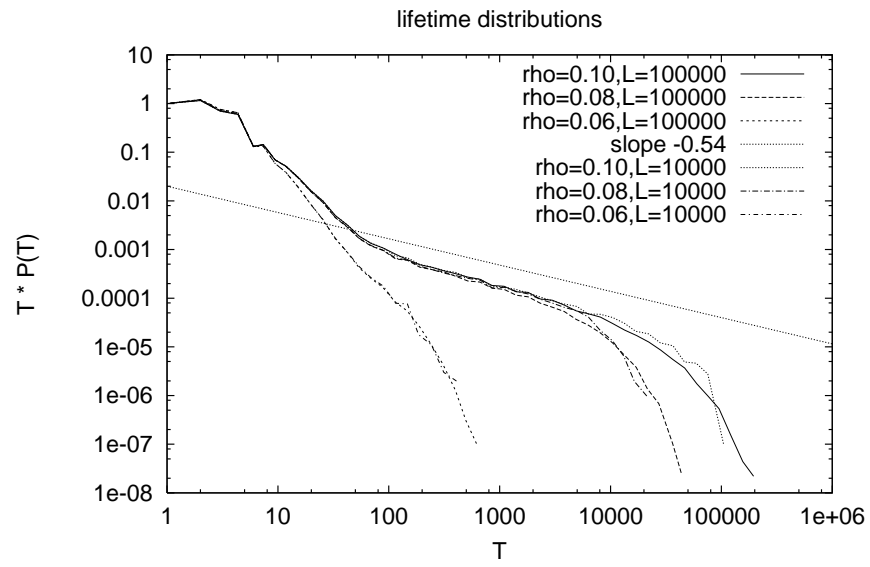

Fig. 28. Lifetime distribution in the NaSch model for $v_{\max }=5$ and $p=0.5$ and various densities below and above $c_{m} \approx 0.085$.

Fig. 28 shows the results of Monte Carlo simulations for the lifetime distribution in the NaSch model for different densities near the transition region, $c \approx c_{m}=0.085 \pm 0.005$ (for $v_{\max }=5, p=0.5$ ), where $c_{m}$ is the density where the flow is maximal. The most interesting feature of the lifetime distribution is the existence of a cutoff near $\tau_{c}=10000$. It has been shown [151] that this cutoff is neither a finite-size nor a finite-time effect. For times smaller than $\tau_{c}$ a scaling regime exists where the distribution decays algebraically.

- Dissolution of a megajam

Gerwinski and Krug [150] tried to find an intuitive criterion which allows the distinction of free-flow and jammed phases. It is based on the investigation of jam dissolution times. Starting from a megajam configuration, i.e. a block of $N$ consecutive cells occupied by vehicles with the remaining $L-N$ cells being empty, they determined the time until the jam ${ }^{14}$ has dissolved completely.

A simple estimate gives the density at which the lifetime is expected to become infinite. Suppose that the jam dissolves with velocity $v_{J}$. Since the first vehicle

${ }^{14}$ In [150] the same definition of a jam as in the previous point "Distribution of lifetimes of jams" (see [151]) has been used. 
move freely with average velocity $v_{F}=v_{\max }-p$ it will reach the end of the jam at the same time as the dissolution wave if the condition $(L-N) / v_{F}=N / v_{J}$ is satisfied. The corresponding density is then given by

$$
c^{*}=\frac{v_{J}}{v_{J}+v_{F}}=\frac{v_{J}}{v_{J}+v_{\max }-p} .
$$

For $v_{\max }=1$ vehicles accelerate immediately to $v_{\max }$. In this case one has $v_{J}=q=1-p$. For higher velocities, $q=1-p$ is only an upper bound for $v_{J}$. Inserting $v_{J}=1-p$ into (82) one therefore obtains an upper bound for the density $c^{*}$. Taking into account interactions between vehicles in the outflow region of the jam, one can derive an effective acceleration rate $q_{e f f}$, and thus the jam dissolution velocity $v_{J}=q_{e f f}$, as a function of $p$ [150].

Computer simulations show a sharp increase of the lifetime near the density $c^{*}$. It becomes "infinite", i.e. the jam does not dissolve within the measurement time, only at a higher density $c_{1}^{*}$ which is considerably larger than the density $c_{m}$ of maximum flow. At intermediate densities $c^{*}<c<c_{1}^{*}$ the jam does not dissolve during the first lap, but later due to fluctuations of the two ends of the jam. During this time other jams have usually formed. All results found in [150] are consistent with the measurements of the lifetime distributions presented in the previous point.

\section{- Relaxation time}

A characteristic feature of a second order phase transition is the divergence of the relaxation time at the transition point. For $p=0$ this has been studied first by Nagel and Herrmann [113]. They found a maximum $\tau_{\max }$ of the relaxation time at the density $c=1 /\left(v_{\max }+1\right)$ which diverges in the thermodynamic limit $L \rightarrow \infty$ as $\tau_{\max } \propto L$. For finite $p$ the behaviour of the relaxation time is more complicated. For technical reasons Csányi and Kertész [152] made no direct measurements of the relaxation time, but used the following approach: Starting from a random configuration of cars with velocity $v_{j}=0$ the average velocity $\bar{v}(t)$ is measured at each time step $t$. For $t \rightarrow \infty$ the system reaches a stationary state with average velocity $\left\langle\bar{v}_{\infty}\right\rangle$. The relaxation time is characterised by the parameter [152]

$$
\tau=\int_{0}^{\infty}\left[\min \left\{v^{*}(t),\left\langle\bar{v}_{\infty}\right\rangle\right\}-\langle\bar{v}(t)\rangle\right] d t
$$

$v^{*}(t)$ denotes the average velocity in the acceleration phase $t \rightarrow 0$ for low vehicle density $\rho \rightarrow 0$. In this regime, due to the absence of interactions between the vehicles, one has $v^{*}(t)=(1-p) t$. Thus the relaxation time is obtained by summing up the deviations of the average velocity $\langle\bar{v}(t)\rangle$ from the values

of a system with one single vehicle which can move without interactions with 
other cars $(\rho \rightarrow 0)$. Note that for a purely exponentially decaying quantity $v(t)=v_{\infty}+C \exp \left(-t / \tau^{\prime}\right)$ the definition (83) is proportional to $\tau^{\prime}$, i.e. the standard definition of the relaxation time. One finds a maximum of the relaxation parameter near, but below, the density of maximum flow for $p=0.25$ and $v_{\max }=5$ (see $\left.[152]\right)$.

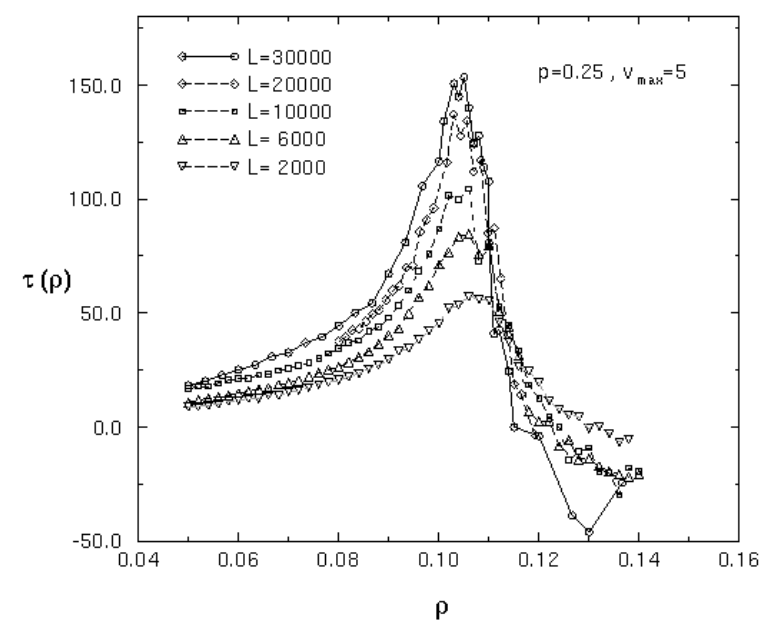

Fig. 29. Relaxation parameter near the transition density for $v_{\max }=5$ and $p=0.25$.

The relaxation time (83) shows interesting behavior [152,139,149] which is difficult to interpret in terms of critical point phenomena. One finds a maximum of the relaxation parameter near, but below, the density of maximal flow. This maximum value increases with system size, but the width of the transition region does not seem to shrink [139]. Two scenarios are possible: 1) The relaxation time converges to a large but finite value for large system sizes beyond the present computer power; 2) The relaxation time diverges for $L \rightarrow \infty$. Scenario 1) appears to be more plausible in view of the finite lifetimes of jams discussed above. Complicated interactions between jams could in principle lead to a divergence. Keeping in mind the unusual scaling behaviour of the width, this should occur in a finite interval, not at a special (critical) point.

The interpretation of the parameter $\tau$ as a relaxation time can be problematic. This can be seen clearly for $c>c_{\text {transition, }}$, where $\tau$ can become negative [149]. Here it is possible that during relaxation the system can temporarily reach states with a higher average velocity than in the stationary state. This overreaction can be divided into two phases for $p>0$. Within the first few time steps small clusters which occur in the initial configuration vanish. The second phase is characterized by the growth of surviving jams. More and more cars get trapped into large jams and therefore the average flow decreases to its stationary value. This decrease causes negative values of $\tau$ at large densities.

- Distribution of distance-headways

In order to get information on the spatial organization of the vehicles, one can 
calculate the distance-headway distribution $\mathcal{P}_{d h}(\Delta x)$ by following either a siteoriented approach [153] or a car-oriented approach [146] if $\Delta x_{j}=x_{j+1}-x_{j}$, i.e., if the number $\Delta x_{j}-1$ of empty lattice sites in front of the $j$-th vehicle is identified as the corresponding distance-headway.

Stated precisely, $\mathcal{P}_{d h}(k)$ is the conditional probability of finding a string of $k$ empty sites in front of a site which is given to be occupied by a vehicle. A comparison between the naive mean-field expression

$$
\mathcal{P}_{d h}^{m f a}(j)=c(1-c)^{j}
$$

for the distance-headway distribution in the NaSch model with $v_{\max }=1$ and the corresponding Monte Carlo data [153] reveals the inadequacy of equation (84) at very short distances which indicates the existence of strong short-range correlations in the NaSch model that are neglected by the mean-field treatment. This is consistent with our earlier observation that there are particlehole effective short-range attraction in the NaSch model with $v_{\max }=1$. Again, this correlation disappears when a random sequential updating is carried out! The exact distance-headway distribution in the NaSch model with $v_{\max }=1$ is found to be $[153,146]$

$$
\mathcal{P}_{d h}^{2 c}(j)=\frac{y^{2}}{c(1-c)}\left[1-\frac{y}{(1-c)}\right]^{j-1} \quad(j=1,2, \ldots)
$$

where $y=P_{2}(1,0)$ is given by the equation (76).
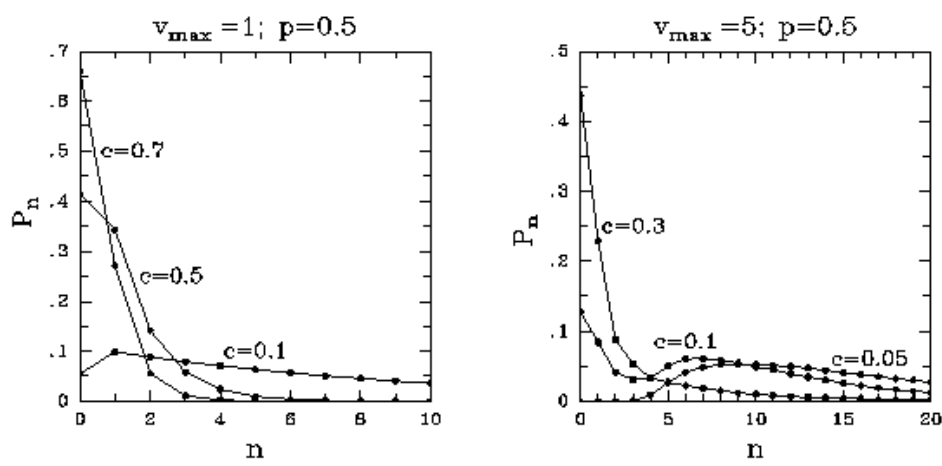

Fig. 30. Distributions of distance-headways in the NaSch model for $p=0.5$ and different densities for (a) $v_{\max }=1$ and (b) $v_{\max }=5$. $n$ denotes the number of empty cells in front of a vehicle and is related to the distance-headway by $n=\Delta x-1$.

For all $v_{\max }>1$, at moderately high densities, $\mathcal{P}_{d h}(\Delta x)$ exhibits two peaks, in contrast to a single peak in the distance-headway distributions for $v_{\max }=1$ at all densities (Fig. 30); the peak at $\Delta x=1$ is caused by the jammed vehicles while that at a larger $\Delta x$ corresponds to the most probable distanceheadway in the free-flowing regions. At first sight, the simultaneous existence of free-flowing and jammed regions may appear analogous to the coexistence of gaseous and liquid phases of matter in equilibrium. In fact, when 
the two-peaked structure of the distance-headway distribution was first observed $[153,115]$, it was erroneously interpreted as a manifestation of the coexistence of two dynamical phases, namely, the free-flowing phase and the jammed phase. But, later works [154] established that the analogy between the coexistence of free-flowing and jammed regions in the NaSch model and the coexistence of the gas and liquid phases of matter cannot be pushed too far because the analogue of the gas-liquid interfacial tension is zero in the NaSch model. Thus, one can not conclude that the NaSch model exhibits a first order dynamical phase transition.

- Distributions of jam sizes and gaps between jams

One can identify a string of $k$ successive stopped vehicles as a jam of length $k$ (by definition, such jams are compact). Similarly, when there are $k$ lattice sites between two successive jams, each occupied by a moving vehicle or is vacant then we say that there is a gap of length $k$ between the two successive jams. Analytical expressions for the distributions of the jam sizes as well as of the gaps between jams can be calculated for the NaSch model (and some of its extensions) using the 2-cluster approximation or or COMF $[143,153,155,156]$. The expressions are exact in the case $v_{\max }=1$ with periodic boundary conditions. For higher velocities the results are only approximative. In COMF the probability $C_{k}$ to find a jam of length $k$ is given by

$$
C_{k}^{(C O M F)}=\left(1-P_{0}\right) P_{0}^{k-1}
$$

whereas in the 2-cluster approach one finds

$$
\begin{aligned}
C_{k}^{(2-c l)} & =\frac{1}{\mathcal{N}_{J}} P(\underline{0} \mid 1) P(\underline{1} \mid 1)^{k-2} P(\underline{1} \mid 1) P(\underline{1} \mid 0) \quad(k \geq 2), \\
C_{1}^{(2-c l)} & =\frac{1}{\mathcal{N}_{J}} \sum_{v=1}^{v_{\max }} P(\underline{0} \mid v) P(\underline{v} \mid 0) .
\end{aligned}
$$

For the $n$-cluster approximation similar expressions can be derived.

Both distribitions (86) and (87) decay exponentially for large jam sizes. COMF always predicts a monotonous distribution with $C_{k}^{(C O M F)} \geq C_{k+1}^{(C O M F)}$. In contrast, the jam size distribution in the $n$-cluster approximation can in principle exhibit a maximum at small jam sizes $1 \leq k \leq n$.

- Distribution of time-headways

Since flux is equal to the inverse of the average time-headway, much more detailed information is contained in the full distribution of the time-headway than in the fundamental diagram. The time-headway distribution contains information on the temporal organization of the vehicles. 
Suppose, $\mathcal{P}_{m}\left(t_{1}\right)$ is the probability that the following vehicle takes time $t_{1}$ to reach the detector, moving from its initial position where it was located when the leading vehicle just left the detector site. Suppose, after reaching the detector site, the following vehicle waits there for $\tau-t_{1}$ time steps, either because of the presence of another vehicle in front of it or because of its own random braking; the probability for this event is denoted by $Q\left(\tau-t_{1} \mid t_{1}\right)$. The distribution $\mathcal{P}_{t h}(\tau)$, of the time-headway $\tau$, can be obtained from $[154,157]$

$$
\mathcal{P}_{t h}(\tau)=\sum_{t_{1}=1}^{\tau-1} \mathcal{P}_{m}\left(t_{1}\right) Q\left(\tau-t_{1} \mid t_{1}\right)
$$

Substituting the expressions for $\mathcal{P}_{m}\left(t_{1}\right)$ and $Q\left(\tau-t_{1} \mid t_{1}\right)$ for $v_{\max }=1$ in (88) we, finally, get $[154,157]$

$$
\begin{aligned}
\mathcal{P}^{t h}(t)= & \frac{q y}{c-y}\left(1-\frac{q y}{c}\right)^{t-1}+\frac{q y}{d-y}\left(1-\frac{q y}{d}\right)^{t-1} \\
& -\left[\frac{q y}{c-y}+\frac{q y}{d-y}\right] p^{t-1}-q^{2}(t-1) p^{t-2} .
\end{aligned}
$$

where, $q=1-p, d=1-c$ and, for the given $c$ and $p, y$ can be obtained from equation (77). The expression is plotted in Fig. 31(a) for a few typical values of $c$ for a given $p$. A few typical time-headway distributions in the NaSch model for $v_{\max }>1$, obtained through computer simulation, are shown in Fig. 31(b).
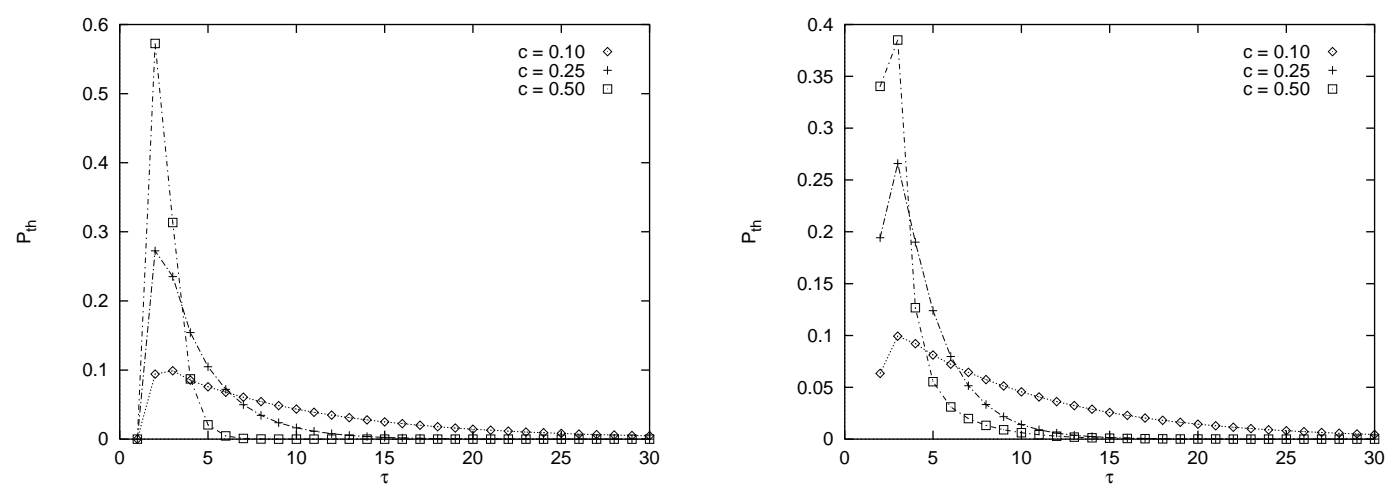

Fig. 31. Time-headway distribution in the NaSch model with (a) $v_{\max }=1$ and (b) $v_{\max }=5$.

- Temporal correlations

In order to probe the spatio-temporal correlations in the fluctuations of the occupation of the cells, one can study the space-time correlation function

$$
C(r, \tau)=\frac{1}{T} \frac{1}{L} \sum_{j=1}^{L} \sum_{t=1}^{T} n_{i}(t) n_{j+r}(t+\tau)-c^{2}
$$


which, by definition, vanishes in the absence of any correlation. In [158] three different regimes have been distinguished.

Free-flow $\left(0<\rho \leq \rho_{1}\right)$ : "Minijams" occur which resolve immediately. The correlation function shows anticorrelations around propagating peak.

Jamming $\left(\rho_{1}<\rho \leq \rho_{2}\right)$ : Free-flow and jamming coexist, i.e. jams with a finite lifetime and vehicles moving with $v_{\max }$ occur. This coexistence is reflected in the behaviour of the correlation function which exhibits a double-peak structure.

Superjamming $\left(\rho_{2}<\rho \leq 1\right)$ : The whole system is congested. Jamming waves are connected and form an infinite wave. As a consequence, the propagating peak in the correlation function has disappeared.

Neubert et al. [159] have introduced a special autocorrelation function of the density in order to study the velocity of jams. They have determined jam velocities for several variants of the NaSch model which will be introducted in later sections.

- Structure factor

Structure factors are known to give valuable information about driven systems [14]. For the NaSch model the static structure factor

$$
S(k)=\frac{1}{L}\left\langle\left|\sum_{j=1}^{L} n_{j} e^{i k j}\right|\right\rangle
$$

has been investigated in [160]. Again $n_{j}$ denotes the occupation number of cell $j$. Note that $S(k)$ is related to the Fourier transform of the density-density correlation function (80).

For all densities, $S(k)$ exhibits a maximum at $k_{0} \approx 0.72$ which corresponds to the characteristic wavelength $\lambda_{0}=2 \pi / k_{0}$ of the density fluctuations in the free-flow regime. For $v_{\max }>1$ one finds $k_{0}\left(v_{\max }+1\right)=$ const.

In [161] these investigations have been extended to the dynamical structure factor in velocity-space,

$$
S_{v}(k, \omega)=\frac{1}{N T}\left\langle\left|\sum_{n, t} v_{n}(t) e^{i(k n-\omega t)}\right|^{2}\right\rangle,
$$

with $k=2 \pi m_{k} / N, \omega=2 \pi m_{\omega} / T$, where $N$ is the number of vehicles and $m_{k}$ and $m_{\omega}$ are integers. $v_{n}(t)$ is the velocity of the $n$-th vehicle at time $t$.

Compared to the dynamical structure factor in real space, (92) has the advantage that the free-flow regime only contributes white noise, $\left.S_{v}(k, \omega)\right|_{\text {free-flow }}=$ const. Therefore it is easier to study jamming properties. It is found in [161] 
that $S_{v}(k, \omega)$ exhibits one ridge with negative slope, corresponding to backward moving jams. One finds that the velocity of the jams is a function of the randomization parameter $p$ only. It is independent of the density $c$ and the maximal velocity $v_{\max }$ [161]. This is consistent with results from a direct study of the autocorrelation function [159] ${ }^{\square}$. Above a transition density, an algebraic behavior $\left.S_{v}(k, \omega)\right|_{\omega / k=v_{\mathrm{j}}} \sim k^{-\gamma}$ of the structure factor is found. This has been interpreted as an indication of critical behavior in [161]. However, due to the difficulties involved in the calculation of (92) only relatively short times $T \leq 2048$ have been considered in [161]. This is much smaller than the cutoff found in the lifetime of jams (see the discussion above) and lies well in the region where an algebraic decay is found. Therefore the results for the dynamical structure factor (92) and the lifetime measurements are consistent, but the algebraic decay is not to be interpreted as an evidence for the existence of a critical point in the NaSch model. In order to see the cutoff, times $T>10^{4}$ would have to be considered.

\subsection{Exact solution of the NaSch model with $v_{\max }=1$ and open boundary conditions}

The analytical methods presented in Section 8.3 are well suited for the investigation of translationally-invariant stationary states which are achieved by imposing periodic boundary conditions. For both practical and theoretical reasons sometimes different boundary conditions are preferable. Imagine a situation where a multilane road is reduced to one lane, e.g. due to road construction. Such a bottleneck can be modeled by using a NaSch model with open boundaries. The multilane part of the road acts as a particle reservoir. If the first cell of the one-lane part is empty a car is inserted here with probability $\alpha$. At the other end a car is removed from the last cell with probability $\beta$ (see Fig. 32). These boundary conditions break the translational invariance and in general one can expect stationary states with a non-trivial density profile $\left\langle\tau_{j}\right\rangle$. From a more theoretical point of view such models have been studied intensively as prototypes of systems exhibiting so-called boundary-induced phase transitions $[162,163]$. In contrast to what one expects from experience with equilibrium systems, one-dimensional driven nonequilibrium systems can exhibit phase transitions, even when the interactions are short-ranged, just by 'slightly' changing the boundary conditions.

The probability distribution characterizing the steady state of the TASEP with parallel dynamics (i.e., NaSch model with $v_{\max }=1$ ) and open boundary conditions has been obtained recently in [164] and [165] using generalizations

\footnotetext{
${ }^{15}$ Measurements of the jam dissolution speed in [150], however, show a decrease with increasing $v_{\max }$ and saturation for large $v_{\max }$.
} 


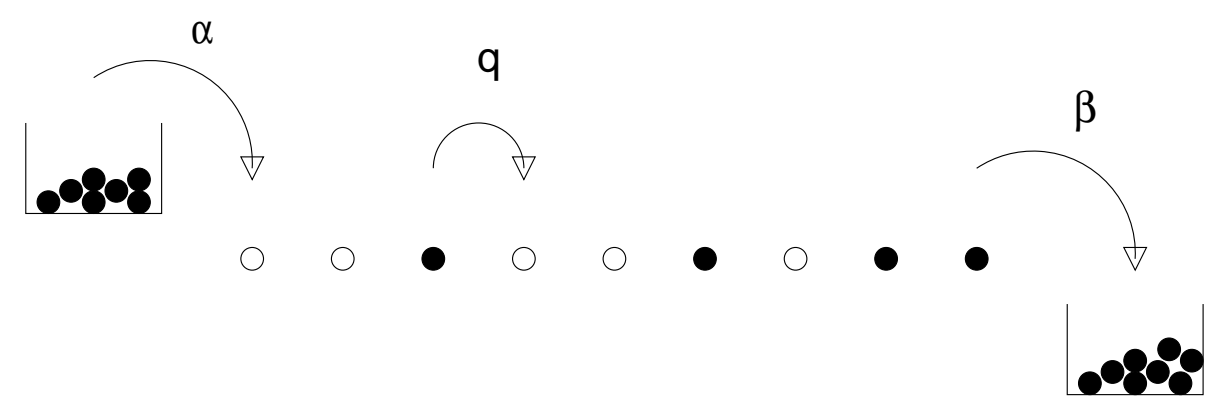

Fig. 32. Definition of the ASEP with open boundary conditions.

of techniques based on the Matrix Product Ansatz (MPA) (see Appendix F for a more technical introduction). By varying the boundary rates $\alpha$ and $\beta$ one obtains a surprisingly rich phase diagram (see Fig. 33) which is qualitatively the same for all types of dynamics. Three phases can be distinguished by the functional dependence of the current through the system on the system parameters. In the low-density phase $\mathrm{A}\left(\alpha<\beta, \alpha_{c}(p)\right)$ the current is independent of $\beta$. Here the current is limited by the rate $\alpha$ which then dominates the behaviour of the system. In the high-density phase $\mathrm{B}\left(\beta<\alpha, \beta_{c}(p)\right)$ the behaviour is dominated by the output rate $\beta$ and the current is independent of $\alpha$. In the maximum current phase $\mathrm{C}\left(\alpha>\alpha_{c}(p)\right.$ and $\left.\beta>\beta_{c}(p)\right)$ the limiting factor for the current is the bulk rate $q=1-p$. Here the current becomes independent of both $\alpha$ and $\beta$.

High- and low-density phase can be subdivided into two phases AI, AII and BI and BII, respectively. These subphases can be distinguished by the asymptotic behaviour of the density profiles at the boundaries.

The transitions between the phases can be characterized by the behaviour of two correlation lengths $\xi_{\alpha}$ and $\xi_{\beta}$ which only depend on $p$ and $\alpha$ or $\beta$. These lengths can be obtained explicitly from the exact solution. Apart from $\xi_{\alpha}$ and $\xi_{\beta}$ also a third length $\xi^{-1}=\left|\xi_{\alpha}^{-1}-\xi_{\beta}^{-1}\right|$ plays an important role.

The transition from AII (BII) to C is continuous with diverging correlation length $\xi_{\alpha}\left(\xi_{\beta}\right)$. The transition from the high- to the low-density phase is of first order. Here both $\xi_{\alpha}$ and $\xi_{\beta}$ are finite, but $\xi$ diverges. On the transition line one finds a linear density profile created by the diffusion of a domain wall between a low-density region at the left end of the chain and a high-density region at the right end.

For the case of parallel dynamics, i.e. the NaSch model with $v_{\max }=1$, the

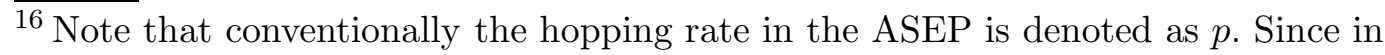
the NaSch model $p$ is the braking probability the hopping rate in the ASEP (for $v_{\max }=1$ ) becomes $q=1-p$.
} 


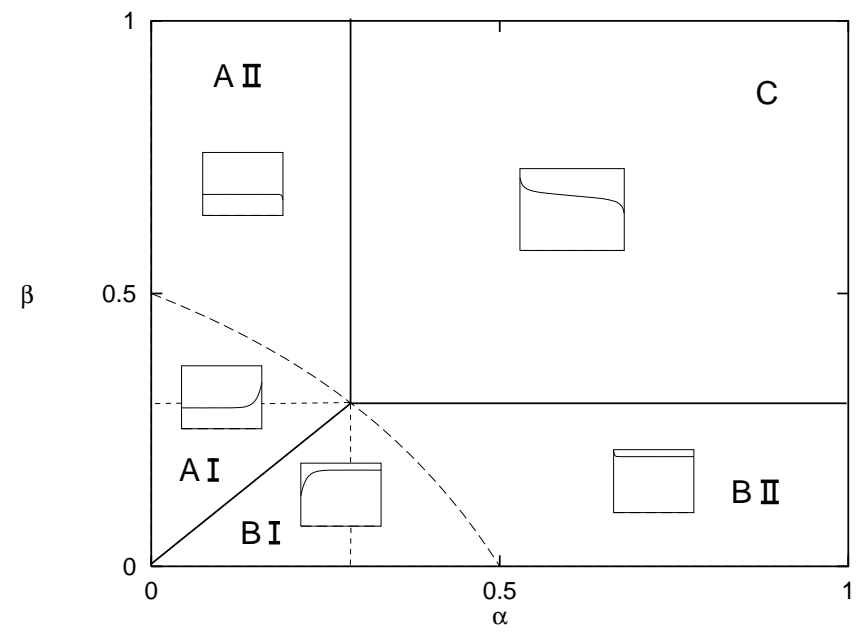

Fig. 33. Phase diagram of the ASEP for parallel dynamics. The inserts show typical density profiles.

currents in the three phases are given by [164-166]

$$
J_{A}=\frac{\alpha(q-\alpha)}{q-\alpha^{2}}, \quad J_{B}=\frac{\beta(q-\beta)}{q-\beta^{2}}, \quad J_{C}=\frac{1}{2}(1-\sqrt{p}) .
$$

The corresponding bulk densities ${ }^{17}$ are

$$
c_{A}=\frac{\alpha(1-\alpha)}{q-\alpha^{2}}, \quad c_{B}=\frac{q-\beta}{q-\beta^{2}}, \quad c_{C}=\frac{1}{2} .
$$

The phase boundaries are determined by the critical rates

$$
\alpha_{c}(p)=\beta_{c}(p)=1-\sqrt{p} .
$$

In Fig. 33 also the special line $(1-\alpha)(1-\beta)=p$ is indicated. Here the density profile is flat (i.e. constant). On this line the exact solution can be obtained by the 2-cluster approach of Sec. 8.3.3 [166]. Since it goes through all three phase these results are sufficient to obtain exact analytic expressions, e.g. for the currents, once the structure of the phase diagram is established (e.g. by Monte Carlo simulations).

The stationary state of the ASEP can also be obtained for other types of updates (see App. A), e.g. random-sequential [167,168], ordered-sequential $[166,169,170]$ and sublattice-parallel update $[171-173,166]$. One finds that the phase diagram has the same basic structure for all updates [166]. The functional dependence of the currents, density profiles etc. on the model parameters differs, however. For the important case of random-sequential updating

${ }^{17}$ In the maximum current phase no real bulk density can be defined due to the algebraic behaviour of the density profile. $c_{C}$ is therefore just $\left\langle\tau_{L / 2}\right\rangle$. 
(93)-(95) have to be replaced by $[167,168]$

$$
\begin{aligned}
J_{A} & =q \alpha(1-\alpha), & J_{B}=q \beta(1-\beta), & J_{C}=\frac{q}{4}, \\
\rho_{A} & =\alpha, & \rho_{B}=1-\beta, & \rho_{C}=\frac{1}{2}, \\
\alpha_{c}(p) & =\beta_{c}(p)=\frac{q}{2} . & &
\end{aligned}
$$

Results for other updates can be found in [166]. For a discussion of the calculation of diffision constants and shock profiles we refer to the reviews $[22,174]$ and references therein.

In [162] the behaviour of the ASEP for $\beta=1$ was explained by postulating a maximal-current principle. According to this principle, independent of the details of the dynamics, the system tries to maximize the stationary current $J$ :

$$
J=\max _{c \in\left[0, c_{-}\right]} J(c)
$$

Here $J(c)$ is the fundamental diagram (for periodic boundary conditions) and $c_{-}$is the density at the left (input) boundary, i.e. $c_{-}=\alpha$ in the case described above.

In [175] a nice physical picture has been developed which explains the structure of the phase diagram not only qualitatively, but also (at least partially) quantitatively. It is determined by the dynamics of a domain walls ${ }^{18}$. In nonequilibrium systems, a domain wall is an object connecting two possible stationary states. The notion of domain walls in the ASEP can be illustrated in the limit $\alpha L \ll 1$ and $\beta L \ll 1$ of small boundary rates. At late times there will be a low-density region at the left end of the chain and a high-density region at the right end, with a domain wall in between. Schematically this state can be depicted as 000011111. For general values of the rates the wall not be sharp in general, but spread over a few lattice sites.

For late times the dynamics of the system can then be interpreted in terms of the motion of the domain wall. A particle entering the system leads moves the wall one cell to left, and a particle leaving the system moves it one cell to the right. Therefore the domain wall performs a biased random walk with drift velocity $v_{D}=\beta-\alpha$ and diffusion coefficient $D=(\alpha+\beta) / 2$. For $\alpha<\beta$ the domain wall moves to the right until it reaches the end of the system which is thereafter in the low-density stationary state. For $\alpha>\beta$ the wall moves to the left until it reaches the left end and the systems goes into the high-density

${ }^{18} \mathrm{~A}$ somewhat related approach has been used to obtain an approximate solution for the special case of parallel dynamics with deterministic bulk dynamics $(p=0)$ $[176]$. 
stationary state. In the case $\alpha=\beta$ there is no net drift in the position of the wall. It fluctuates with its rms displacement increasing with time as $(D t)^{1 / 2}$, i.e. it can be anywhere in the system resulting in a linear density profile.

In order to understand the case of general $\alpha$ and $\beta$ one has to introduce a second kind of domain wall separating a maximum current phase from the high-density phase. Since the maximal possible flow for periodic boundary conditions is $J_{\max }=1 / 4$ (for $p=0$ and random-sequential update) the dynamics for $\alpha=1 / 2$ is dominated by the overfeeding at the left boundary. The injection rate could support a current larger than $1 / 4$, but in the bulk it can not exceed this value. Therefore at the left boundary a maximum current state is formed. If the particles are not extracted fast enough at the right boundary a high-density region will develop there. These two regions are separated by a new kind of domain wall, the maximum current/high-density domain wall. Schematically it can be represented as mmmm1111. Again this domain wall performs a biased random walk.

In order to obtain more quantitative predictions one goes to a coarse-grained picture. Then it is useful to replace the boundary rates $\alpha$ and $\beta$ by particle reservoirs with densities $c_{-}$and $c_{+}$. The continuity equation $\partial c / \partial t+\partial J / \partial x=0$ in the continuum limit has traveling wave solutions of the form $c\left(x-v_{D} t\right)$ with the domain wall velocity

$$
v_{D}=\frac{J_{+}-J_{-}}{c_{+}-c_{-}}
$$

which can be obtained by integration over the chain.

For the low-density/high-density domain wall one has 19 $J\left(c_{+}\right)=\beta(1-\beta)$ and $c_{-}=\alpha, J_{-}=J\left(c_{-}\right)=\alpha(1-\alpha)$. This gives indeed $v_{D}=$ $\beta-\alpha$ which should be valid for $\alpha, \beta<1 / 2$. For the maximum current/highdensity domain wall $c_{-}$takes the value $c_{-}=1 / 2$ so that $J_{-}=1 / 4$ and thus $v_{D}=\beta-1 / 2$.

The arguments described above can be generalized to any process where the fundamental diagram $J(c)$ of the periodic system has only one maximum at a density $c^{*}$. For all currents $J<J\left(c^{*}\right)$ there exist two corresponding densities $c_{1}$ and $c_{2}$ with $J\left(c_{1}\right)=J=J\left(c_{2}\right)$. For fundamental diagrams with more than one maximum, more than two densities might exist for a given current $J$. This implies the existence of a larger number of domain wall types. The phase diagram of the open system than exhibits a larger number of phases [177]. The maximal-current principle (99) for the TASEP with $\beta=1$ is generalized

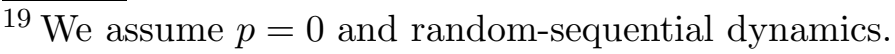


to the extremal-current principle $[177,15]$

$$
J= \begin{cases}\max _{c \in\left[c_{+}, c_{-}\right]} J(c) & \text { for } c_{-}>c_{+}, \\ \min _{c \in\left[c_{-}, c_{+}\right]} J(c) & \text { for } c_{-}<c_{+} .\end{cases}
$$

Since the above phenomenological picture does not depend on the microscopic details of the dynamics, it plausible that the phase diagrams for different updates are qualitatively the same. Boundary-induced phase transitions have recently been observed [178] in measurements on a German motorway [41]. One finds a first-order nonequilibrium phase transition between a free-flow and a congested phase. This transition is induced by the interplay between density waves induced by an on-ramp and a shock wave moving on the motorway [178].

\section{Generalizations and extensions of the NaSch model}

As stated earlier, the NaSch model is a minimal model. The first obvious possible generalization would be to replace the acceleration stage of updating rule (U1) to

$$
v_{n} \rightarrow \min \left(v_{n}+a_{n}, v_{\max }\right)
$$

where, $a_{n}$, acceleration assigned to the $n$-th vehicle, need not be unity and, in general, may depend on $n$. In the following subsections we consider more non-trivial generalizations and extensions of the NaSch model.

\subsection{Single-lane highways}

In the next few subsubsections we shall demonstrate the rich variety of traffic phenomena that can be observed by appropriate modifications of the random braking. We have earlier mentioned in the context of empirical results that traffic flow exhibits metastability and the related hysteresis effects. Such phenomena have been observed in continuum formulations of "microscopic" models, i.e., in coupled-map lattice models [115]. However, the NaSch model is too simple to account for these phenomena. We now briefly describe a few generalizations of the NaSch model, each of which is based on modifications of the braking rules of the original NaSch model; one common feature of all of these generalized models is that they show metastability and hysteresis.

Before we begin our discussions on specific generalized versions of the NaSch model, which exhibit metastability, we make some general remarks. In the schematic stationary fundamental diagram of Fig. 34, the low density branch 


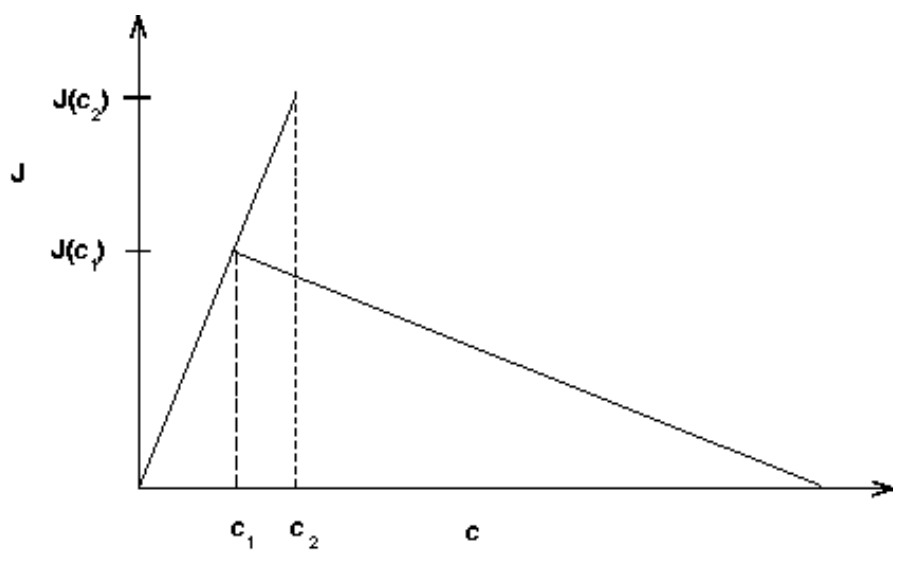

Fig. 34. Metastability and hysteresis.

corresponds to homogeneous free-flow states, while the high density branch corresponds to configurations, where jammed states are present. Obviously, at densities $c_{1}<c<c_{2}$, the flow depends non-uniquely on the global density.

In order to establish the existence of meta-stable states one can follow two basic strategies. In the first method, the density of vehicles is changed adiabatically by adding or removing vehicles from the stationary state at a certain density. Starting in the jamming phase (large densities) and removing vehicles, one obtains the lower branch of the hysteresis curve. On the other hand, by adding vehicles to a free flowing state (low densities), one obtains the upper branch. This method is closely related to the experimental situation, where the occupancy of the road varies continuously.

The second method does not require changing the density. Instead one starts from two different initial conditions, the mega-jam and the homogeneous state. The mega-jam consists of one large, compact cluster of standing vehicles. In the homogeneous state, vehicles are distributed periodically with same constant gap between successive vehicles (with one larger gap for incommensurate densities). Then, for $c>c_{1}$ the homogeneous initialization leads to a freeflow state, while the mega-jam initialization leads to the jammed high-density states.

\subsubsection{Cruise-control limit and self-organized criticality}

In the cruise-control limit of the NaSch model [179] vehicles moving with their desired velocity $v_{\max }$ are not subject to noise. This is exactly the effect of a cruise-control which automatically keeps the velocity constant at a desired value. In this model the acceleration, deceleration (due to other vehicles) and movement stages of updating are identical to those in the general case of the NaSch model; however, the randomization step is applied only to vehicles which have a velocity $v<v_{\max }$ after step 2 of the update rule. We can express 
this more formally by recasting the randomization stage of the update rules in the NaSch model as follows:

$$
v_{n} \rightarrow \max (0, v-1)
$$

with probability

$$
p= \begin{cases}p_{v_{\max }} & \text { if } v=v_{\max } \\ p & \text { if } v<v_{\max }\end{cases}
$$

where $v$ is the velocity of the vehicle at the end of the step 2 of the update rule, i.e., after deceleration due to blocking by other vehicles. In the original formulation of the NaSch model $p_{v_{\max }}=p$. On the other hand, the cruisecontrol limit corresponds to $p_{v_{\max }} \rightarrow 0, p \neq 0$.

For $p_{v_{\max }} \ll 1$, at sufficiently low densities, all the vehicles move deterministically with the velocity $v_{\max }$; this deterministic motion is, however, interrupted by small perturbations at a vanishingly small rate. Consequently, the system gets enough time to relax back to the state corresponding to the deterministic algorithm before it is perturbed again. This effectively separates completely the time scales for perturbing the system and the response of the system.

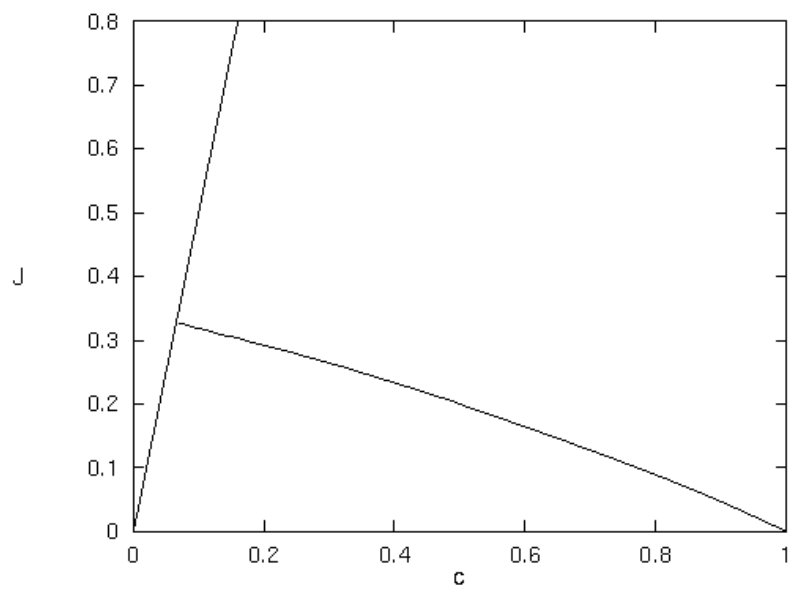

Fig. 35. Fundamental diagram in the cruise-control limit of the NaSch model for $v_{\max }=5$ and $p_{v_{\max }}=0, p=0.5$.

First, let us consider the periodic boundary conditions which is easier to treat than than the open boundary conditions. In this model, a sharp transition from the "free-flowing" dynamical phase to the "congested" phase takes place at a critical concentration $c_{*}\left(p, v_{\max }\right)$ which depends on $p$ as well as $v_{\max }$ and, for all $p \neq 0, c_{*}\left(p, v_{\max }\right)$ is smaller than $c_{m}^{\text {det }}=1 /\left(v_{\max }+1\right)$. For a given $v_{\max }, c_{*}(p)$ increases with decreasing $p$ and, in the deterministic limit $p \rightarrow 0$, $c_{*}\left(0, v_{\max }\right) \rightarrow c_{m}^{\text {det }}$.

In this model a jam is defined to consist of vehicles all of which have their instantaneous velocities smaller than $v_{\max }$. For all $c<c_{*}$, jams present in 
the initial configuration eventually disappear and in the jam-free stationary state every vehicle moves with the velocity $v_{\max }$. Therefore, in the density regime $c<c_{*}$ the flux increases linearly with density following $J=c v_{\max }$, just like that in the deterministic limit $p=0$ of the NaSch model (Fig. 35). But, unlike the deterministic limit $p=0$, the cruise-control limit of the NaSch model exhibits metastability in the interval $c_{*}<c<c_{m}^{d e t}$. In this context, the metastability means that, in the interval $c_{*}<c<c_{m}^{d e t}$, on appropriate initialization, the system can reach apparently steady states where no jam appears and where the fluxes are higher than $J\left(c_{*}\right)$; but, perturbations of such a "metastable" state creates long-lived jams thereby reducing the flux to a level consistent with the stable branch of the fundamental diagram. At all $c>c_{*}$ jams present in the initial configuration never disappear completely and, in this density regime, the stable steady-state is a mixture of laminar flow regions and jams. The long-lived jams lower the flux beyond $c_{*}$ and the flux decreases linearly with density (Fig. 35).

Let us assume that at densities slightly above $c_{*}$, only one jam of length $L_{\text {jam }}$ containing $N_{\text {jam }}$ vehicles exists in the system. Then, because of the periodic boundary conditions, the total number of vehicles $N$ is conserved and, hence,

$$
N=c_{\text {jam }} L_{\text {jam }}+c_{\text {out }}\left(L-L_{\text {jam }}\right)
$$

where $c_{j a m}=N_{j a m} / L_{j a m}$ and $c_{\text {out }}=\left(N-N_{\text {jam }}\right) /\left(L-L_{j a m}\right)$ are the densities of the vehicles in the jam and in the outflow region, respectively. Dividing both sides of (103) by $L$ we get

$$
c=c_{j a m} \frac{L_{j a m}}{L}+c_{\text {out }}\left(1-\frac{L_{\text {jam }}}{L}\right)
$$

Since in the cruise-control limit of the NaSch model $L_{j a m}$ must vanish as $c \rightarrow c_{*}$, we conclude [179] that we must have $c_{\text {out }}=c_{*}$, i.e., the average density in the outflow region of a jam is equal to the critical density $c_{*}$.

In order to study the traffic at the critical point of the cruise-control limit, Nagel and Paczuski [179] used a a special boundary condition which enables the system to select automatically the state of maximum throughput, i.e, the system exhibits self-organized criticality. This special boundary condition consists of an infinite jam from $-\infty$ to 0 (i.e., at the left boundary) while the right boundary is open. Vehicles emerge from the infinite jam in a jerky way, before attaining the velocity $v_{\max }$. Far away from the infinite jam all vehicles move with the same velocity $v_{\text {max }}$. In order to show that the state selected this way is "critical" [179] we perturb a vehicle, far downstream from the infinite jam, slightly by reducing its velocity from $v_{\max }$ to $v_{\max }-1$. This particular vehicle initiates a chain reaction and gives rise to a jam if the following vehicle is sufficiently close to it although it itself accelerates and, eventually, attains $v_{\max }$. This phantom jam has a time-dependent size $n(t)$, measured by the 
number of vehicles $n$ in this jam at time $t$ and it has a lifetime $\tau_{\text {life }}$. The statistics of this features of the phantom jam can be obtained by repeating the computer experiment sufficiently large number of times; sometimes the phantom jam created is small and has a short lifetime and sometimes it is large and has quite long lifetime. Interestingly, the characteristic quantities like, for example, the distributions of the sizes of the jams, lifetimes of the jams, etc. do, indeed, exhibit power-laws which are hall mark of the self-organized criticality $[26,27]$. E.g. the branching behaviour of the jams gives rise to intermittent dynamics with a $1 / f$ power law spectrum [179]. $1 / f$ noise in real traffic has been discovered by Musha and Higuchi [180]. They recorded transit times of vehicles passing underneath a bridge. The corresponding power spectral density of the flow fluctuations shows $1 / f$ behaviour at low frequencies.

The exponents associated with the various power laws in the cruise-control limit of the NaSch model can be calculated analytically, at least for $v_{\max }=1$, by utilizing a formal relation with one-dimensional unbiased random walk. If $v_{\max }=1$, all the vehicles in the jams have velocity $v=0$. Moreover, the jams are compact so that the number of vehicles in a jam is identical to its spatial extent. The probability distribution $P(n ; t)$ for the number of vehicles $n$ in such a jam, at time $t$, is determined by the following equation:

$$
P(n ; t+1)=\left(1-r_{\text {in }}-r_{\text {out }}\right) P(n ; t)+r_{\text {in }} P(n-1 ; t)+r_{\text {out }} P(n+1 ; t)
$$

where the phenomenological parameters $r_{\text {in }}$ and $r_{\text {out }}$ are the rates of incoming and outgoing vehicles. Of course, $r_{i n}$ depends on the density of the vehicles behind the jam. For large $n$ and $t$, taking the continuum limit of the equation (105) and expanding we get

$$
\frac{\partial P}{\partial t}=\left(r_{\text {out }}-r_{\text {in }}\right) \frac{\partial P}{\partial n}+\frac{r_{\text {in }}+r_{\text {out }}}{2} \frac{\partial^{2} P}{\partial n^{2}}
$$

If $r_{\text {in }}>r_{\text {out }}$ the jams would grow for ever. On the other hand, the jams would shrink, and eventually disappear, if $r_{\text {in }}<r_{\text {out }}$. If $r_{\text {in }}=r_{\text {out }}$, the first term on the right hand side of the equation (106) vanishes and the resulting equation governing the time evolution of $P(n ; t)$ is identical to that of the probability of finding, at time $t$, an unbiased one-dimensional random walker at a distance $n$ which was initially at the origin. Thus, when $r_{i n}=r_{\text {out }}$, the jams exhibit large ("critical") fluctuations which can be characterized by critical exponents. Using this formal mapping onto unbiased random walk, we find (a) that the mean size of jam at time $t$ corresponds to the mean displacement of the random walker from the origin after time interval $t$ and (b) that the lifetime of a jam corresponds to the time taken by the random walker to return to the origin for the first time. Hence, using the well known results from the theory of random walks $[181,182]$, we get

$$
n(t) \propto t^{1 / 2}, \quad \text { and } \quad P\left(\tau_{\text {life }}\right) \propto \tau_{\text {life }}^{-3 / 2} .
$$


It turns out that the power-law exhibited by the size of the jams, the distributions of the lifetimes, etc. are not restricted merely to the special case $v_{\max }=1$ of the cruise-control limit but is also shown by the corresponding computer simulation data also for arbitrary $v_{\max }$. The power-law distributions of $P\left(\tau_{\text {life }}\right)$ in the cruise-control limit of the NaSch model is in sharp contrast with the exponential distribution observed in the NaSch model [151]. Thus, in the cruise-control limit of the NaSch model, the large jams are fractal [183] in the sense that there are smaller sub-jams inside larger jams, ad infinitum. In other words, in between sub-jams, there are holes of all sizes.

\subsubsection{Slow-to-start rules, metastability and hysteresis}

The slow-to-start rules can lead not only to metastability and, consequently, hysteresis, but also to phase separated states at high densities, as we now show.

- Takayasu-Takayasu slow-to-start rule

Takayasu and Takayasu (TT) [184] were the first to suggest a CA model with a slow-to-start rule. Here, we investigate the generalization, as suggested in [185], of the original slow-to-start rule. According to this generalized version, a standing vehicle (i.e., a vehicle with the instantaneous velocity $v=0$ ) with exactly one empty cell in front accelerates with probability $q_{t}=1-p_{t}$, while all other vehicles accelerate deterministically. The other steps of the update rule (U2-U4) of the NaSch model are kept unchanged.

As in the case of the NaSch model, it is instructive to consider first the deterministic limits of the TT model $[184,186]$. The TT model reduces to the NaSch model in the limit $p_{t}=0$. What happens in the other deterministic limit, namely, $p_{t}=1$ ? In the latter deterministic limit, a stopped vehicle can move only if there are at least two empty cells in front [184]. Obviously, completely blocked states exist for densities $c \geq 0.5$, where the number of empty cells in front of each vehicle is smaller than two. However, in the region $0.5 \leq c \lesssim 0.66$ the number of blocked configurations is very small compared to the total number of configurations and states with a finite flow exist. Precisely at $c=0.5$, there are only two blocked states and the time to reach these states diverges exponentially with the system size.

The fundamental diagram for the TT model with $v_{\max }=1$ has been derived analytically by carrying out (approximate) 2-cluster calculations in the siteoriented approach [185]. But, the fundamental diagrams of the TT model for all $v_{\max }>1$ have been obtained so far only numerically by carrying out computer simulations (see Fig. 36).

Comparing these fundamental diagrams with the corresponding ones for the 


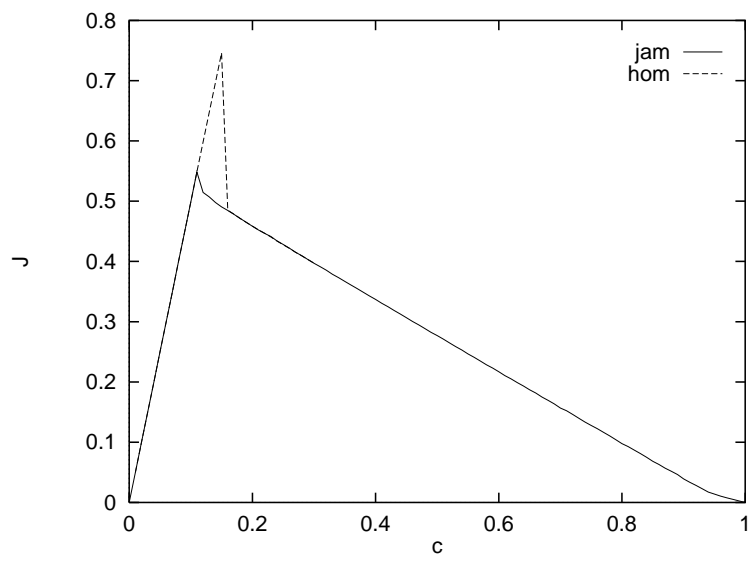

Fig. 36. The fundamental diagram in the TT model $\left(v_{\max }=5\right.$, $\left.p=0.01, p_{t}=0.75, L=10000\right)$ obtained using two different initial conditions, namely, a completely jammed state (jam) and a homogeneous state (hom) and averaging over 100000 sweeps through the lattice.

NaSch model $\left(p_{t}=0\right)$, we find the following effects of the TT slow-to-start rule: (i) for a given density $c$, the flux $J(c)$ is smaller in the TT model as compared to that in the NaSch model; (ii) the particle-hole symmetry is not exhibited by the TT model for any $v_{\max }$ (not even for $v_{\max }=1$ ) and (iii) the TT model exhibits metastability and hysteresis which are absent in the NaSch model. Note that the mechanism for meta-stability in the case $p_{t}=1$ is different from that for the metastability observed for $0<p_{t}<1$.

Because of the slow-to-start rules, the separations between the vehicles coming out of a jam are larger than those between the vehicles coming out a jam in the NaSch model. Since the density far downstream is smaller than the density of maximum flow, the vehicles can propagate freely in the low density regions of the lattice where spontaneous formation of jams is highly unlikely, if the parameter $p$ is sufficiently small. Therefore, the phase-separated steady-states at high global densities consist of a macroscopic jam and a macroscopic freeflow regime both of which coexist simultaneously (Fig. 37).

- The BJH model of slow-to-start rule

Benjamin, Johnson and Hui (BJH) [187] modified the updating rules of the NaSch model by introducing an extra step where their "slow-to-start" rule is implemented; this slow-to-start rule is different from that introduced by TT [184]. According the BJH "slow-to-start" rule, the vehicles which had to brake due to the next vehicle ahead will move on the next opportunity only with probability $1-p_{s}$. The steps of the update rules can be stated as follows:

Step 1: Acceleration. $v_{n} \rightarrow \min \left(v_{n}+1, v_{\max }\right)$.

Step 2: Slow-to-start rule: If $f l a g=1$, then $v_{n} \rightarrow 0$ with probability $p_{s}$. 


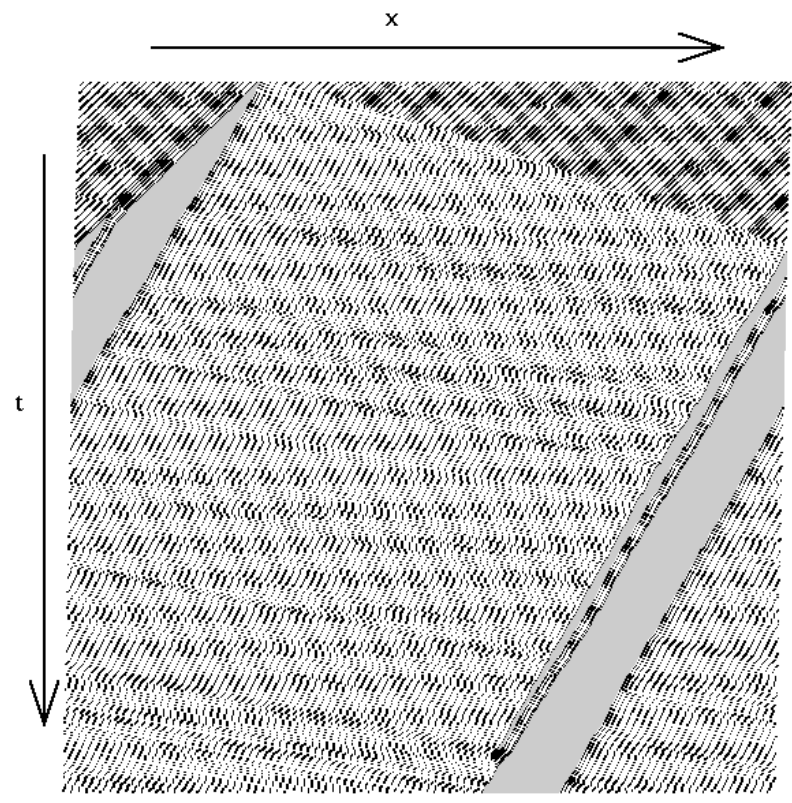

Fig. 37. Typical space-time diagram of the TT model with $v_{\max }=5$ and $c=0.20$, $p=0.01$ and $p_{t}=0.75$. Each horizontal row of dots represents the instantaneous positions of the vehicles moving towards right while the successive rows of dots represent the positions of the same vehicles at the successive time steps.

Step 3: Blockage (due to other vehicles). $v_{n} \rightarrow \min \left(v_{n}, d_{n}-1\right)$ and, then, flag $=1$ if $v_{n}=0$, else flag $=0$.

Step 4: Randomization. $v_{n} \rightarrow \max \left(v_{n}-1,0\right)$ with probability $p$.

Step 5: Vehicle movement. $x_{n} \rightarrow x_{n}+v_{n}$.

Here flag is a label distinguishing vehicles which have to obey the slow-tostart rule $($ flag $=1)$ from those which do not have to $($ flag $=0)$.

Obviously, for $p_{s}=0$ the above rules reduce to those of the NaSch model. The slow-to-start rule of the TT model is a 'spatial' rule. In contrast, the BJH slow-to-start rule requires 'memory', i.e. it is a 'temporal' rule depending on the number of trials and not on the free space available in front of the vehicle. The fundamental diagram of the BJH model (Fig. 38) clearly shows the existence of metastable states and, therefore, expected to exhibit hysteresis effects [155]. But, in the special case of $v_{\max }=1$, for which approximate analytical calculations can be carried out [185], no meta-stable states exist.

Since for all $v_{\max }>1$ in the BJH model, just as in the TT model, the outflow from a jam is smaller than the maximal flow, the phase-separated steadystates at high global densities consist of a macroscopic jam and a macroscopic free-flow regime both of which coexist simultaneously (Fig. 39) [155]. 


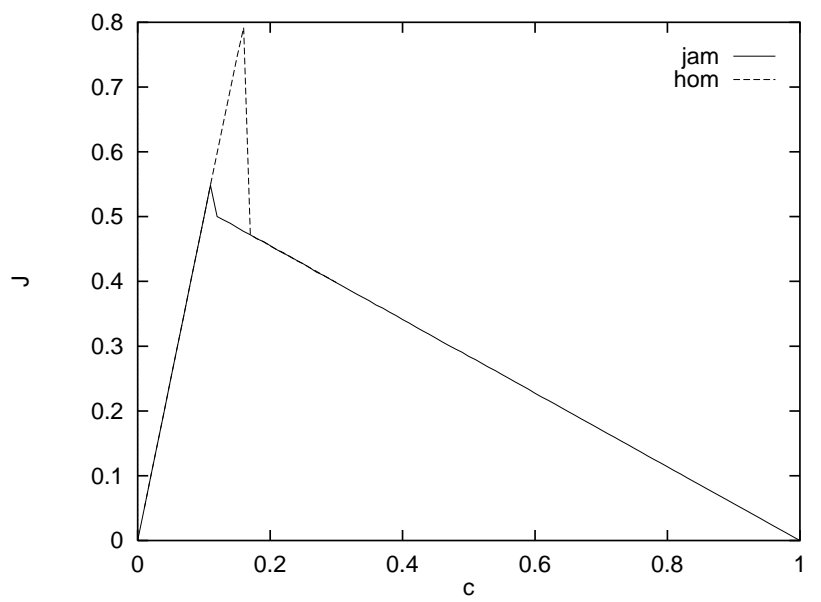

Fig. 38. The fundamental diagram in the BJH model $\left(v_{\max }=5, p=0.01, p_{s}=0.75\right)$ obtained using two different initial conditions, namely, a completely jammed state (jam) and a homogeneous state (hom).

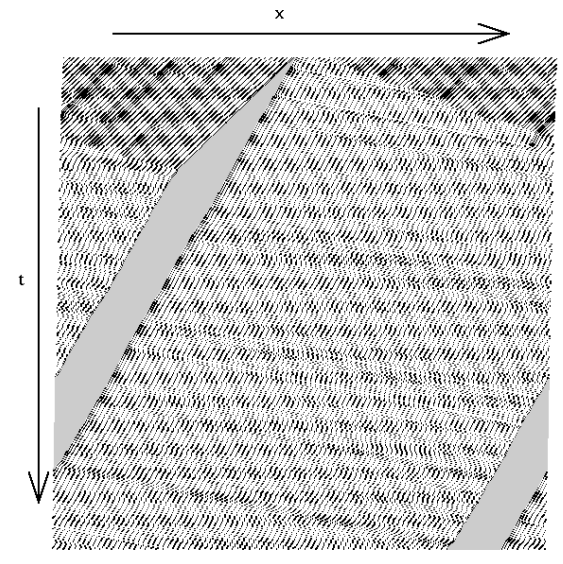

Fig. 39. Typical space-time diagram of the BJH model with $v_{\max }=5$ and $c=0.20$, $p=0.01$ and $p_{s}=0.75$. Each horizontal row of dots represents the instantaneous positions of the vehicles moving towards right while the successive rows of dots represent the positions of the same vehicles at the successive time steps.

However, the macroscopic jam is not compact. The typical size of the macroscopic free-flow regime can be estimated by measuring the distribution of the gaps between the successive jams [155]. A peak occurs in this distribution for headways of the order of the system size (see the inset of the right part of Fig. 40). The position of the peak indicates the typical size of the macroscopic free-flow regime.

- The NaSch model with a velocity-dependent slow-to-start rule

Although the NaSch model does not exhibit metastable states and hysteresis, a simple generalization exists which is able to reproduce these effects. It is the so-called Velocity-Dependent-Randomization (VDR) model [188]. Here, in 


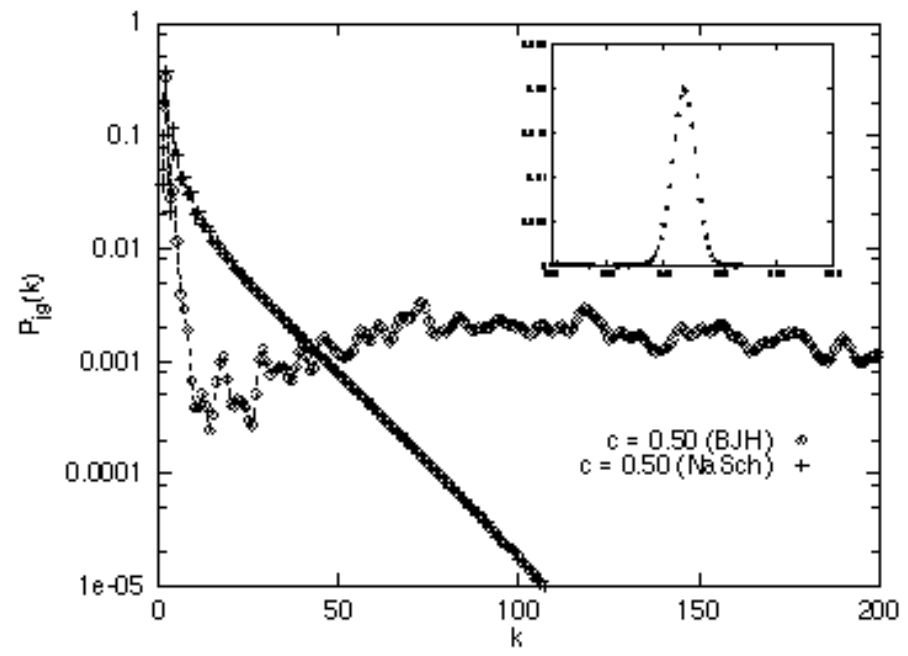

Fig. 40. Distribution of gaps between jams in the BJH and the NaSch model for $v_{\max }=5, p=0.05, p_{s}=0.5$ and density $c=0.5$.

contrast to the original NaSch model, the randomization parameter depends on the velocity of the vehicle, $p=p(v)$. The rules (see Sec. 8) are supplemented by a new rule,

Step 0: Determination of the randomization parameter. The randomization parameter used in step 3 for the $n$-th vehicle is given by $p=p\left(v_{n}(t)\right)$.

This new step has to be carried out before the acceleration step 1 . The randomization parameter used in step 3 depends on the velocity $v_{n}(t)$ of the $n$-th vehicle after the previous timestep. In order to implement a simple slow-tostart rule one chooses [188]

$$
p(v)= \begin{cases}p_{0} & \text { for } v=0 \\ p & \text { for } v>0\end{cases}
$$

with $p_{0}>p$. This means that vehicles which have been standing in the previous timestep have a higher probability $p_{0}$ of braking in the randomization step than moving vehicles.

The rules of the VDR model can be recast in a form similar to those of the BJH model. We define a label flag which distinguishes between vehicles which have to obey the slow-to-start rule $($ flag $=1)$ from those which do not have to $(f l a g=0)$. flag $=1$ if $v_{n}=0$ at the beginning of a time step, else flag $=0$. Explicitly, the update rules are as follows:

Step 1: Acceleration. $v_{n} \rightarrow \min \left(v_{n}+1, v_{\max }\right)$.

Step 2: Blockage (due to other vehicles). $v_{n} \rightarrow \min \left(v_{n}, d_{n}-1\right)$, 
Step 3: Randomization. $v_{n} \rightarrow \max \left(v_{n}-1,0\right)$ with probability $p_{0}$ if flag $=1$. else, $v_{n} \rightarrow \max \left(v_{n}-1,0\right)$ with probability $p$.

Step 4: Vehicle movement. $x_{n} \rightarrow x_{n}+v_{n}$.

Let us compare this VDR model with the cruise-control limit of the NaSch model. The vehicles with velocity $v=v_{\max }$ (at the end of the step 2) are treated deterministically in the cruise-control limit whereas in the VDR model velocities of all those with the velocity $v>0$ (just before the step 3) are updated stochastically, but using different values of the braking parameter.

Typical fundamental diagrams look like the one shown in Fig. 41 where, over a certain interval of $c, J(c)$ can take one of the two values depending on the initial state and, therefore, exhibit metastability.

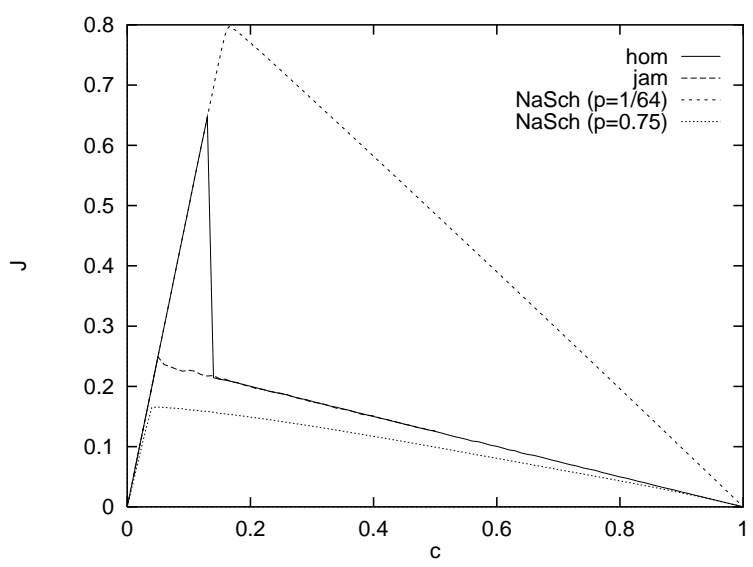

Fig. 41. The fundamental diagram in the NaSch model with a velocity-dependent slow-to-start rule $\left(v_{\max }=5, p_{0}=0.75, p=1 / 64\right)$ obtained using two different initial conditions, namely, a completely jammed state (jam) and a homogeneous state (hom).

Moreover, typical space-time diagrams of the VDR model (see Fig.42) clearly demonstrate that metastable homogeneous states have a lifetime after which their decay leads to a phase separated steady state. The microscopic structure of these phase-separated high-density states is qualitative similar to those observed in the high-density regimes of the TT and BJH models but differs drastically from those found in the NaSch model.

It is instructive to compare the fundamental diagram of the VDR model with those of the corresponding NaSch models. We now present a simple derivation of the fundamental diagram of the VDR model on the basis of heuristic arguments utilizing the observed structures of the steady-states. For small densities $c \ll 1$ there are no slow vehicles in the VDR model since interactions between vehicles are extremely rare. In this regime every vehicle can move with the free-flow velocity $v_{f}=(1-p) v_{\max }+p\left(v_{\max }-1\right)=v_{\max }-p$ and, therefore, 


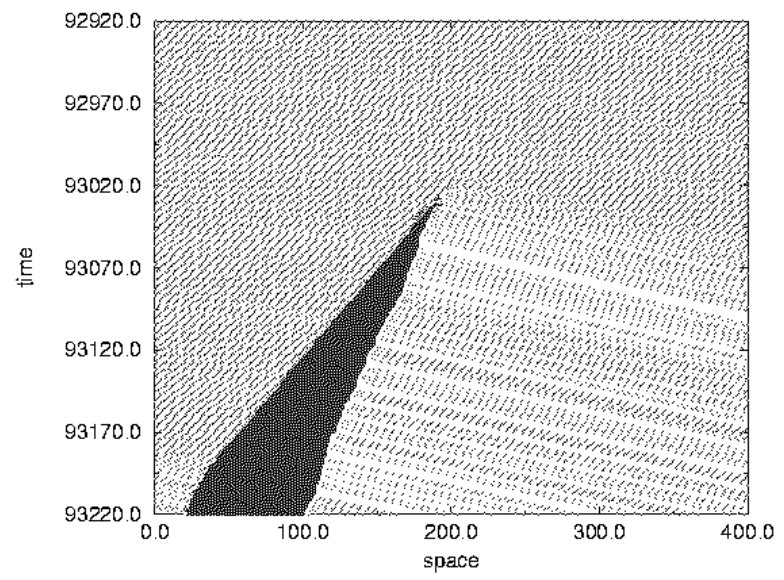

Fig. 42. Typical space-time diagram of the VDR model with $v_{\max }=5$ and $c=0.20$, $p=0.01$ and $p_{0}=0.75$. Each horizontal row of dots represents the instantaneous positions of the vehicles moving towards right while the successive rows of dots represent the positions of the same vehicles at the successive time steps.

the flux is given by

$$
J_{\text {hom }}(c)=c\left(v_{\max }-p\right)
$$

which is identical to the NaSch model with randomization $p$. On the other hand, for densities close to $c=1$, the vehicles are likely to have velocities $v=0$ or $v=1$ only and, therefore, the random braking is dominated by $p_{0}$, rather than $p$, while the flow is determined by the movement of the holes. Hence, for large densities, i.e., $1-c \ll 1$, the flow is given by $J(c) \approx\left(1-p_{0}\right)(1-c)$ which corresponds to the NaSch model with randomization $p_{0}$. This expression for flux in the high-density regime can also be derived as follows. In the phaseseparated state the vehicles are expected to move with the velocity $v_{f}=$ $v_{\max }-p$ in the free-flow region. Neglecting interactions between vehicles in the free-flowing region (which is justified because of the corresponding low density), the average distance of two consecutive vehicles in the free-flow region is given by $\Delta x=c_{f}^{-1}=T_{w} v_{f}+1$ where the average waiting time $T_{w}$ of the first vehicle at the head of the megajam is given by $T_{w}=\frac{1}{1-p_{0}}$. In other words, the density in the free-flow regime $c_{f}$ is determined by the average waiting time $T_{w}$ and $v_{f}$. Now suppose that $N_{J}$ and $N_{F}$ are the number of vehicles in the megajam and free-flowing regions, respectively. Using the normalization $L=N_{J}+N_{F} \Delta x$ we find that for the density $c=\frac{N_{F}+N_{J}}{L}$, the flux $J_{s e p}(c)$ is given by $J_{\text {sep }}(c)=\frac{N_{F}}{L}\left(v_{\max }-p\right)$ and, hence,

$$
J_{\text {sep }}(c)=\left(1-p_{0}\right)(1-c)
$$

Obviously, $c_{f}$ is precisely the lower branching density $c_{1}$, because for densities below $c_{f}$ the jam-length is zero. It should be noted that the heuristic arguments 
presented above remain valid for $p_{0} \gg p$ and $v_{\max }>1$. The condition $p \ll 1$ guarantees that the jams are compact in that limit. In the case $v_{\max }=1$, vehicles can stop spontaneously, even in the free-flow regime and these vehicles might initiate a jam. This is the basic reason why hysteresis is usually not observed for $v_{\max }=1$.

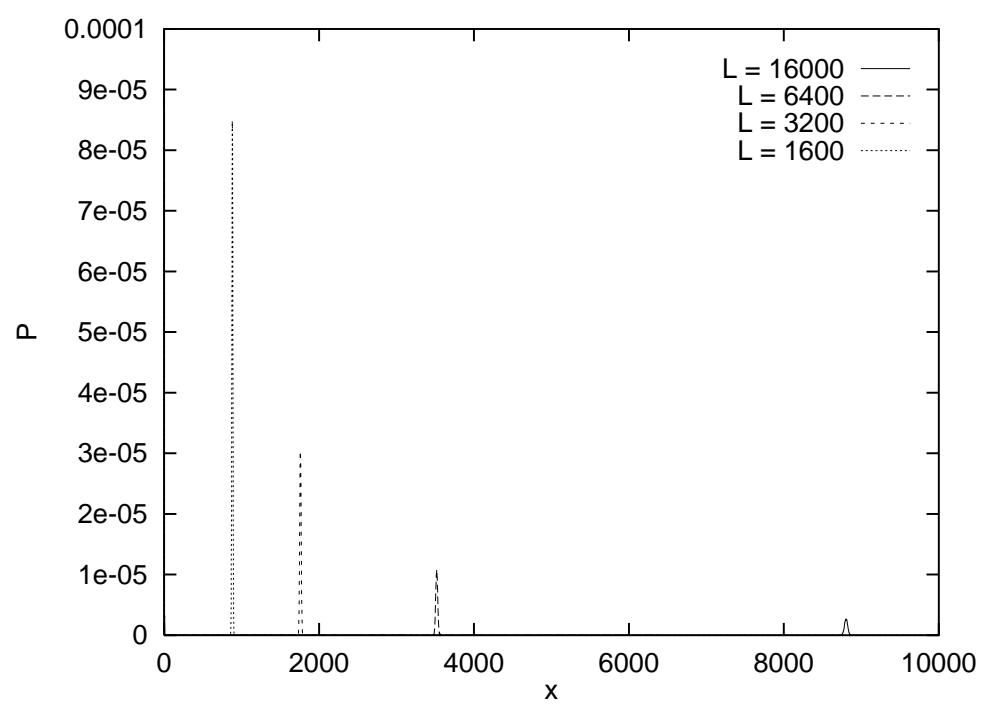

Fig. 43. Peaks of the jam-gap distribution at macroscopic distances.

Analogous to the BJH model phase separation can be directly identified using the results of the jam-gap distribution. Fig. 43 shows that the size of the free-flow regime is proportional to the system size.

The results for the slow-to-start models discussed above have been obtained by computer simulations of periodic systems of finite length. It was shown that the fundamental diagram which is sketched in Fig. 34 is generic for all models under consideration. Now it is self-evident to ask what kind of stationary states are realised in the thermodynamic limit $L \rightarrow \infty$. The simulation results indicate that $\Delta c=c_{1}-c_{2}$ decreases with larger system sizes and is expected to vanish for $L \rightarrow \infty$, i.e. the jammed branch is stable in that limit. This is readily understood if one analyses the typical configurations which lead to an emerging jam or, vice versa, the mechanism of the dissolution of a jam. Jams emerge if overreactions of drivers lead to a chain reaction. This is possible in dense regions of the free-flow state where the gap between the vehicles is not larger than $v_{\max }$. Obviously the probability to find large platoons of vehicles driving with small spatial headways increases with the system size (for fixed density). In addition to that the jammed states are phase separated, i.e. the size of the jam is of the order of the system size. During a simulation run the size of the jam fluctuates due to the stochastic movement and acceleration of the vehicles. Jams can dissolve if the amplitude of these fluctuations are of the order of the length of the jam, which is impossible in the thermodynamic limit. 
Therefore the non-unique behaviour of the fundamental diagram is only observable if finite system sizes are considered or if the vehicles move deterministically in the free flow regime. Nevertheless the results discussed above are highly relevant for practical purposes, because the hysteresis effects have been observed at realistic system sizes (e.g. $L=10000$ corresponds to a highway of length $75 \mathrm{~km}$ ).

\subsubsection{Flow-optimization and meta-stable states}

Hysteresis effects and meta-stable states are not only of theoretical interest, but also have interesting applications. From the previous discussion of the slow-to-start models it is evident, that one can optimize the maximum flow, if the homogeneous state is stabilized by controlling the density so that it never exceeds $c_{2}$. This strategy was followed in minimizing frequent jams in the Lincoln- and the Holland-Tunnels in New York. Before the traffic lights were installed at the entrance of the tunnels jams used to form spontaneously within the tunnel because (a) the vehicle density used to be sufficiently high and (b) the drivers used to drive more carefully inside the tunnel thereby giving rise to stronger fluctuations which caused the jams. But, the traffic lights installed at the entrance of the tunnels do not allow the density to exceed $c_{2}$ and, consequently, jams are not formed spontaneously by the decay of any metastable high-density state.

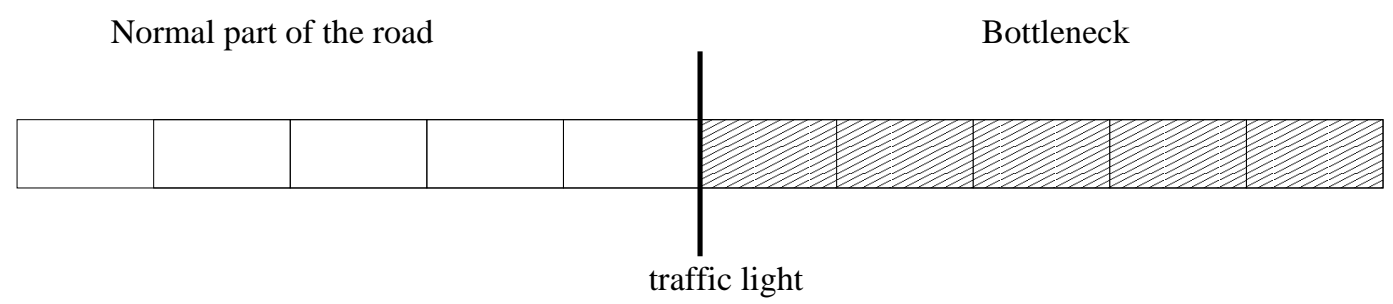

Fig. 44. A schematic representation of a tunnel on a highway. Larger values of the braking parameters are used for the right part, which mimics the tunnel.

One can mimic the situation of Lincoln and Holland-tunnels within the framework of the CA models in the following way [188-190]. The tunnel is considered as part of the road, where the braking probabilities $p^{t}, p_{0}^{t}$ are higher compared to the remaining part of the lattice ( $p, p_{0}$, see Fig. 44$)$. Therefore, if one allows for an uncontrolled inflow of the vehicles, jams typically appear inside the "tunnel" and the system capacity is governed by $p_{0}^{t}$.

The situation differs drastically if traffic lights are implemented [188-190]. As shown in Fig. 45, a considerable increase of the maximum capacity can be achieved for an optimal combination of the red-/green-signal periods. The gain of capacity obtained for the optimal intervals of the signal is of the same order as for the realistic examples [191]. 


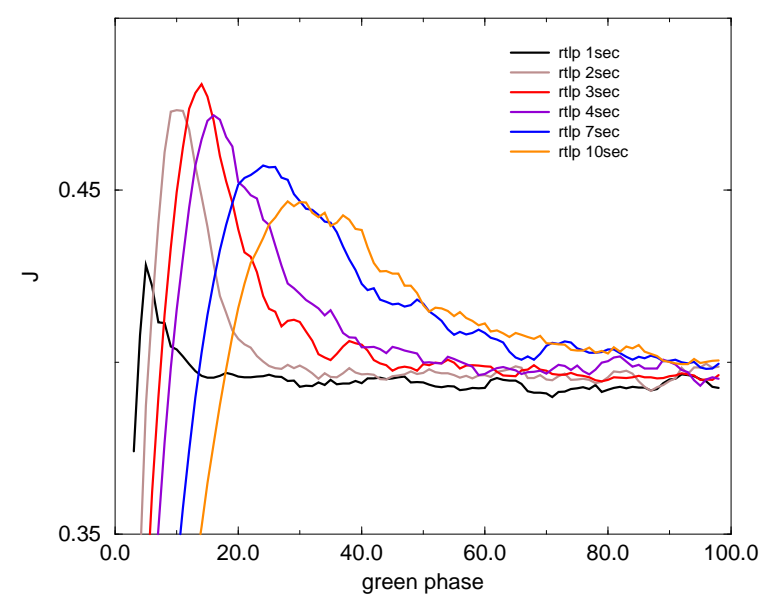

Fig. 45. Average flow for different combinations of red-/green-signal periods for a system of length $L=1000$ and $v_{\max }=5$. Inside the tunnel $p^{t}=0.15, p_{0}^{t}=0.60$ and outside $p=0.01, p_{0}=0.20$ has been used.

\subsection{Multi-lane highways}

For a realistic description of traffic on highways the idealized single-lane models must be generalized to develop CA models of multi-lane traffic; the main ingredient required for this generalization being the lane-changing rules. Several attempts have been made so far in this direction [192-199]. The lane changing rules for two-lane traffic can be symmetric or asymmetric with respect to the lanes. Similarly, if there are two (or more) different types of vehicles (say, cars and trucks) with two different $v_{\text {max }}$, the lane-changing rule can be symmetric or asymmetric with respect to the vehicles.

In general, the update in the two-lane models is divided into two sub-steps: in one sub-step, the vehicles may change lanes in parallel following the lanechanging rules and in the other sub-step each vehicle may move forward effectively as in the single-lane NaSch model. Drivers must find some incentive in changing the lane. Two obvious incentives are (a) the situation on the other lane is more convenient for driving, and (b) the need to make a turn in near future. Two general prerequisites have to be fulfilled in order to initiate a lane change: first, there must be an incentive and second, the safety rules must be fulfilled [200]. Lane changing rules according to this scheme have been introduced by Rickert et al. [193]. They suggested that vehicles are allowed to change the lane if the following four criteria are satisfied:
(C1) $\operatorname{gap}(i)<l$,
(C2) $\operatorname{gap}_{o}(i)>l_{o}$ 
(C3) $\operatorname{gap}_{o, b a c k}(i)>l_{o, b a c k}$

(C4) $\operatorname{rand}()<p_{c}$.

Here $\operatorname{gap}(i)$ and $\operatorname{gap}_{o}(i)$ are the gaps in front of vehicle $i$ on the own lane and the other lane 20 , respectively. $\operatorname{gap}_{o, b a c k}(i)$ is the gap on the other lane to the next vehicle behind. $l, l_{o}, l_{o, \text { back }}$ and $p_{c}$ are parameters specifying the rule and $\operatorname{rand}()$ is a random number in the interval $[0,1]$.

The first rule $\mathbf{C 1}$ represents the incentive criterion, i.e. if the gap gap $(i)$ in front of the vehicle is not sufficiently large vehicles want to change the lane. Typical choices of the parameter $l$ are given by $l=\min \left(v+1, v_{\max }\right)$. This choice of the minimal headway ensures that vehicles driving in a slow platoon try to change the lane if possible. In the next rule C2 it is checked if the situation on the other lane is indeed more convenient. This motivates the choice $l=l_{o}$. The third rule $\mathbf{C 3}$ avoids too small distances to following vehicles on the other lane. Rickert and coworkers suggested $l_{o, b a c k}=v_{\max }$. It is also important to perform lane changing stochastically. Even if the incentive and safety criteria are fulfilled a lane change is performed only with probability $p_{c}(\mathbf{C 4})$. This avoids, at least partially, so called ping-pong lane changes, i.e. multiple lanechanges of vehicles in consecutive timesteps ${ }^{21}$. Already implementations of the NaSch model using the basic lane-changing rules revealed quite realistic results. Nevertheless several variants of the basic rules have been developed in order to improve the realism. A large number of lane changing rules considered in the literature have been tabulated and compared by Nagel et al. [196] (see Fig. 46).

The lane changing rules for two-lane traffic can be symmetric or asymmetric with respect to the lanes [193]. If symmetric lane changing rules are applied the rules do not depend on the direction of the lane changing maneuver. In contrast also asymmetric lane changing rules have been considered. Lane changing rules can be asymmetric in two ways. First it is possible that it is preferred to drive on the right lane at low densities. This behavior can be implemented simply by leaving out the first rule for a change from the left to the right lane. Second it is also possible that it is even forbidden to overtake a vehicle on the right lane, e.g. on german highways. Then the single lane dynamics on the right lane depends on the configuration on the left lane. These examples show the flexibility of the CA approaches. Moreover the simulations also show that the details of the lane changing rules may lead to considerable changes of the model results $[194,196]$.

In multi-lane traffic it is of particular interest to investigate systems with differ-

\footnotetext{
${ }^{20}$ The gap on the other lane is defined in the same way as the gap on the own lane by imagining that the vehicle occupies the site parallel to its current position.

${ }^{21}$ This artifact of the parallel update was already pointed out by Nagatani [192], who simulated a two-lane system with $v_{\max }=1$.
} 


\begin{tabular}{|c|c|}
\hline $\begin{array}{l}\text { Schütt }[14]: \\
R \rightarrow L: v_{r}<v(\text { look-ahead distance }=9) \\
L \rightarrow R: v_{r} \geq v(\text { look-ahead distance }=15)\end{array}$ & {$\left[-v_{\max }+1, v_{\max }\right]$} \\
\hline 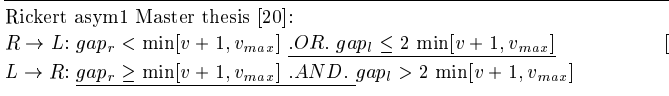 & {$\left[-v_{\max }, \min \left(v+1, v_{\max }\right)\right]$} \\
\hline 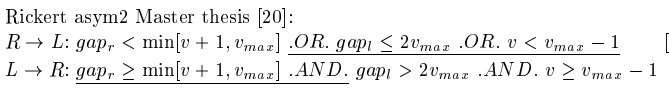 & {$\left[-v_{\max }, \min \left(v+1, v_{\max }\right)\right]$} \\
\hline $\begin{array}{l}\text { Latour } 1 \text { Master thesis [23]: } \\
R \rightarrow L: \operatorname{gap}_{r}<v \\
L \rightarrow R: \operatorname{gap}_{r} \geq v\end{array}$ & {$[0,0]$ (i.e. neighbor cell empty) } \\
\hline 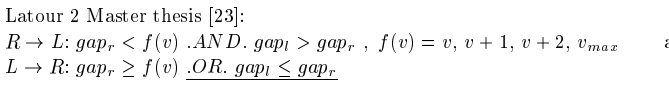 & a) $[0,0]$ b) $[-2,0]$ \\
\hline $\begin{array}{l}\text { Rickert et al Physica A }[21]: \\
R \rightarrow L: \operatorname{gap}_{r}<v+1 \\
L \rightarrow R: \operatorname{gap}_{r} \geq v+1\end{array}$ & {$\left[-\left(v_{\max }+1\right), v+1\right]$} \\
\hline 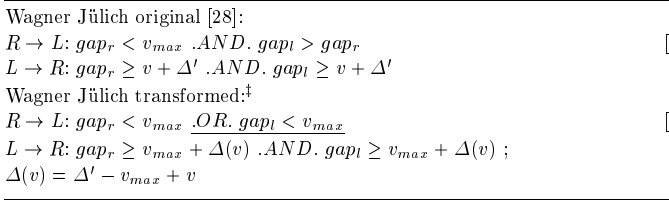 & $\begin{array}{l}{\left[-\left(v_{b a c k}+1\right), 0\right]} \\
{\left[-\left(v_{b a c k}+1\right), \min \left(g a p+1, v_{\max }\right)\right]}\end{array}$ \\
\hline 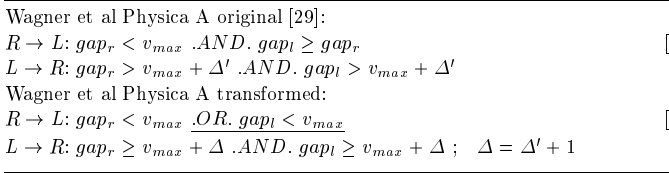 & $\begin{array}{l}{\left[-v_{\max }, 0\right]} \\
{\left[-v_{\max }, \min \left(\text { gap }, v_{\max }\right)\right]}\end{array}$ \\
\hline $\begin{array}{l}\text { Chowdhury et al Physica A }[30]: \\
R \rightarrow L: \operatorname{gap}_{r}<v . O R . v_{d}>v_{d, r} \quad\left(\text { look-ahead distance } v_{\max }\right) \\
L \rightarrow R: \operatorname{gap}_{r} \geq v \cdot A N D . v_{d} \leq v_{d, r} \quad\left(\text { look-ahead distance } v_{\max }\right) \\
\end{array}$ & $\begin{array}{l}{\left[-v_{d, b a c k}, g a p\right]} \\
{\left[-v_{d, b a c k}, v\right]}\end{array}$ \\
\hline $\begin{array}{l}\text { This paper (velocity): } \\
R \rightarrow L: v_{r} \leq v . O R . v_{l} \leq v(\text { look-ahead distance }=16) \\
L \rightarrow R: v_{r}>v+\Delta . A N D . v_{l}>v+\Delta(\text { look-ahead diglance }=16)\end{array}$ & {$\left[-v_{\max }, v\right]$} \\
\hline $\begin{array}{l}\text { This paper (gap): } \\
R \rightarrow L: \text { gap }_{r}<v_{\max } . \text { OR. gap }<v_{\max } \\
L \rightarrow R: \text { gap }_{r} \geq v_{\max }+\Delta . \text { AND. gapl } \geq v_{\max }+\Delta\end{array}$ & {$\left[-v_{\max }, v\right]$} \\
\hline
\end{tabular}

Fig. 46. The table gives an overview over different choices of the lane changing rules discussed in the literature. The numbers of the references correspond to those in [196]. The first column shows different incentive criteria and the second column denotes the corresponding minimal gap on the other lane. In general the incentive criteria can be different for a change from left to right $(L \rightarrow R)$ and $(R \rightarrow L)$ (from $[196])$.

ent types of vehicles. For CA models this has been done first by Chowdhury et al. [195] who simulated a periodic two-lane system with slow and fast vehicles, i.e. vehicles with different $v_{\max }$. The simulation results have been shown that already for small densities the fast vehicles take on the average the free-flow velocity of the slow vehicles, even if only a small fraction of slow vehicles have been considered. Analogous results have been obtained by Helbing and Huberman [212] who used a different CA model for the in-lane update (see Sec. 11.3 for the definition of the model). In addition to that Nagel et al. [196] have been shown that for a suitable choice of the lane changing rules and different types of vehicles even the phenomenon of "lane inversion" which has been observed at german highways can be reproduced ${ }^{22}$. The results discussed so far show

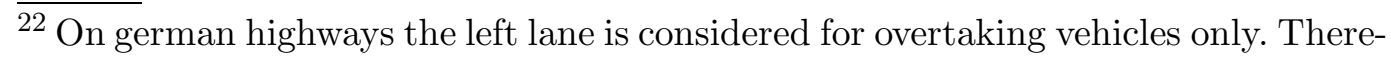


the strong influence of slow vehicles in multi-lane systems. They fit fairly well the empirical results, which show an alignment of the speeds on different lanes and and of different types of vehicles. Nevertheless recent simulation results of Knospe et al. [197] indicate that the influence of slow vehicles seems to be overestimated by the multi-lane variants of the NaSch model. In particular for symmetric lane changing rules even a single slow vehicle can dominate the dynamics close to the optimal value of the flow. In order to weaken the effect of slow vehicles they suggested to consider anticipation effects, i.e. the driver estimates the velocity of the vehicle in the next timestep [197].

Another interesting quantity to look at is the frequency of lane changes at different densities. Here the simulation results show that close to the density of maximal flow the number of lane changing maneuvers drastically decreases if the small values of the braking noise are considered in CA models where the velocities of vehicles are solely determined by the distance to the vehicle ahead. This is due to the fact that for homogeneous states at high densities no sufficiently large gaps exist. For larger values of the braking noise large density fluctuations are observable. Therefore the local minimum of the lane-changing frequency is not found for larger values of $p$.

In general the simulation results show that some generic multi-lane effects can be pointed out. First of all the maximal performance of multi-lane systems is slightly increased compared to corresponding single-lane network. In addition, slow vehicles lead to an alignment of velocities of different type of vehicles already at low densities which is confirmed by empirical observation. This effect is quite robust for different choices of the CA model as well as for different lane changing rules. It can be weakened most efficiently if anticipation effects are applied. The details of the lane changing rules, however, may have strong influence on the lane usage characteristics.

\subsection{Bidirectional traffic}

Simon and Gutowitz [201] have introduced a two-lane CA model where the vehicles move in opposite directions. Passing may be allowed on one or on both lanes. It is only attempted if there is a chance to complete the pass. Therefore drivers measure the local density, i.e. the density of vehicles in front that have to be passed. If it is sufficiently low, a pass will be attempted. This means that at high global densities the lanes are effectively decoupled since only very few passes will occur.

fore, at low densities, the right lane is used more often. Surprisingly, at higher densities not simply a balancing of the lane usage has been observed, but for densities close to the optimum flow the left lane is even higher frequented. 
In principle, three types of jams can occur on a bidirectional road: 1) Spontaneous jamming and start-and-stop waves on one of the lanes; 2) jams caused by drivers who try to pass but can not return to their home lane since there is not enough space and 3) "super jams" when an adjacent pair of drivers tries to pass simultaneously. These super jams halt traffic on both lanes and can be prevented by breaking the symmetry between the lanes.

The precise rules of the CA are in the same spirit as the rules for multilane traffic described in the previous subsection 9.2. First, the situation on the own lane is examined. If the motion is hindered by another vehicle (moving in the same direction), a pass is attempted. This will only be initiated if the safety criteria are satisfied: 1) The gap on the other lane has to be sufficiently large, and 2) the number of vehicles to be passed has to be small. Even if these criteria are satisfied a lane change occurs only with probability $p_{\text {change. After }}$ this lane changing step the vehicles move forward similar to the dynamics of the NaSch model. There are, however, important differences. Passing vehicles never decelerate randomly. In order to break the symmetry between the two lanes moving vehicles which are on their home lane and see oncoming traffic decelerate deterministically by one unit. This rule prevents the occurance of a super jam.

The results of [201] show the expected behaviour, namely that passing makes traffic more fluid. Start-stop waves are surpressed if the density is not too large. The improvement of the flow on one lane compared to the the one-lane model depends on the density of vehicles on the other lane. It is maximal for very small densities $(c \rightarrow 0)$ on the passing lane. If the density on the other lane is small $(c<0.25)$ the flow may be lower than in the one-lane model since passing oncoming vehicles create an additional hindrance. For large densities on at least on of the lanes there is little difference between the one- and twolane models.

Lee et al. [202] have proposed a toy model for bidirectional traffic based on a multispecies generalization of the ASEP. Here no passing is allowed. Instead oncoming traffic on the opposite lane reduces the hopping rates of the vehicles. The dynamics on each lane is given by that of the ASEP with randomsequential update and $v_{\max }=1$, but the hopping rate from an occupied cell $j$ to an empty cell $j+1$ on lane 1 depends on the occupancy of cell $j+1$ on the opposite lane (lane 2). When this cell is empty, vehicles hop with rate 1 , otherwise with rate $1 / \beta$. On lane 2 vehicles move in the opposite direction and the hopping rate from cell $j+1$ to cell $j$ depends on the occupancy of cell $j$ on lane 1 . It is given by $\gamma$ when this cell is empty and by $\gamma / \beta$ if it is occupied.

For $\gamma<1$ the uninfluenced hopping rate on lane 2 is smaller than that of lane 1. The vehicles on lane 2 might therefore be interpreted as trucks. The interlane interaction parameter $\beta$ can be interpreted as a kind of road narrowness. For 
$\beta=1$ vehicles are not slowed down by oncoming traffic. This corresponds to a highway with divider. The case $\beta \rightarrow 0$ corresponds to a narrow road being completely blocked by the oncoming traffic.

The behaviour of the model with only one truck is rather similar to that of the NaSch model with quenched disorder (see Sec. 10). For $\beta>\beta_{c}$ the system segregates into two phases, a high-density phase in front of the truck and a low-density phase behind it.

By forbidding trucks and cars to occupy parallel cell $j$ simultaneously the model can be mapped onto an exactly solvable 2-species variant of the ASEP. Using the matrix-product Ansatz (see App. F) many steady-state properties for the single-truck case can be obtained exactly. Two phase can be distinguished: A low-density phase for $c \beta<1$ and a jammed phase for $c \beta>1$ where $c$ is the density of vehicles on lane 1 . In contrast to the case of a fixed defect site (see Sec. 10.3) only one critical density $c_{\text {crit }}=1 / \beta$ exists since the particle-hole symmetry is broken.

Generalizations of this model to other updates and higher velocities can be found in [203].

\section{Effects of quenched disorder on traffic}

\subsection{Randomness in the braking probability of drivers and Bose-Einstein-like condensation}

We have seen how modifications of the random braking probability or the rule(s) for random braking in the NaSch model can give rise to a rich variety of physical phenomena, e.g., self-organized criticality, metastability and hysteresis, etc. Now we consider the effects of quenched randomness in the random braking probability $p$, i.e., we study the effects of assigning randomly different time-independent braking probabilities $p_{i}$ to different drivers $i$ in the NaSch model. Such "quenched" (i.e., time-independent) randomness in the random braking in the NaSch model can lead to exotic phenomena [204,197] which are reminiscent of "Bose-Einstein-like condensation" in the TASEP where particle-hopping rates are quenched random variables [205,206]. Various aspects of these phenomena have been thoroughly reviewed by Krug [207] and, therefore, we'll restrict our discussion to only the essential points.

Let us first consider the special case of the NaSch model with $v_{\max }=1$. As explained earlier, this model reduces to the TASEP if the parallel updating is replaced by random sequential updating scheme. If the same hopping prob- 
ability $q$ is assigned to every particle except one for which the the hopping probability is $q^{\prime}<q$, then the single "impurity" particle is the slowest moving one. The faster particles can be allowed to overtake the slow one at a non-zero rate [174,208]; however, if this rate of overtaking vanishes the slow particle will give rise to a platoon of particles behind it. This phenomenon is very similar to the formation of platoons of vehicles in a traffic behind the slow vehicles (e.g., trucks).

Here we are interested in a more general situation of quenched "disorder" in the form of a distribution of intrinsic hopping probabilities of the vehicles in the system rather than that of the single "defect" particle. In such situations random initial conditions can lead to the formation of platoons if (a) slow particles are sufficiently rare and (b) if the density of vehicles is sufficiently low. Following their formation, starting from a random initial condition, the platoons grow through coalescence. The coarsening of the platoons has been investigated in the same spirit in which coarsening of domains (the so-called Oswald ripening) is monitored while studying spinodal decomposition in, for example, binary alloys [28]. Suppose, $\xi(t)$ is the typical platoon size at time $t$. Starting from a homogeneous spatial distribution of the vehicles, $\xi(t)$ can be monitored as a function of time $t$ to find out the law of "growth" of the size of the platoons.

Before describing the effects of the quenched randomness in the hopping probabilities on the steady-states of the TASEP and the NaSch model, we consider an even simpler model of platoon formation [209,210] which was developed using the language of aggregation phenomena. In this model an initial velocity $v_{j}$ is assigned to each vehicle $j$, drawn randomly from a continuous probability density $f(v)$. The particles then move ballistically along a line and coalesce whenever a faster vehicle catches up with a slower one in front. It has been found that $\xi(t)$ increases indefinitely according to the power law

$$
\xi(t) \sim t^{(n+1) /(n+2)}
$$

where the exponent $n$ characterizes the behaviour of $f(v)$ in the vicinity of the minimal velocity $v_{\text {min }}$, i.e., $f(v) \sim A\left(v-v_{\text {min }}\right)^{n}$ as $v \rightarrow v_{\text {min }}$ with some positive constant $A$. An attempt has been made to develop a coarse-grained description of this phenomenon [211].

It has been shown $[205,206]$ that if quenched random hopping probabilities are assigned to each particle in the TASEP, there are small gaps between particles in the high-density congested phase but in the inhomogeneous low-density phase there is a macroscopically large empty region in front of the slowest particle (i.e., the particle with smallest hopping probability) behind which a platoon is formed. The phase transition from the low-density inhomogeneous phase (which consists of a macroscopic free region and a platoon) to the highdensity congested phase is, in many respects, analogous to the Bose-Einstein 
transition.

In order to see this analogy, let us imagine that the empty sites are bosons and the state of a boson is determined by which particle it is immediately in front of. In the language of the ideal Bose gas, in the high-density phase the bosons are thinly spread over all the states. On the other hand, in the low-density phase there is a finite fraction of the empty sites are condensed in front of the slowest particle in such "Bose-Einstein-like condensed" state. The steady-state velocity of the particles is the analogue of the fugacity of the ideal Bose gas. What makes the system interesting is the fact that the platoon appears at low-density rather than at high density of the vehicles.

The Bose-Einstein-like-condensation in the TASEP with quenched random hopping probabilities of the individual particles survives when the random sequential updating is replaced by parallel updating [206]. Finally, it is worth emphasizing that, the analogy with the ideal Bose gas is only formal as the empty sites in the TASEP are not non-interacting quantum particles.

The qualitative features of the dynamical phases and phase transitions observed in the NaSch model with random braking probabilities, for $v_{\max }=1$ as well as for larger $v_{\max }$, are very similar to those described above for the TASEP with random hopping probabilities [204]. Typical snapshots of the system at three different stages of evolution from a random initial state are shown in Fig. 47.

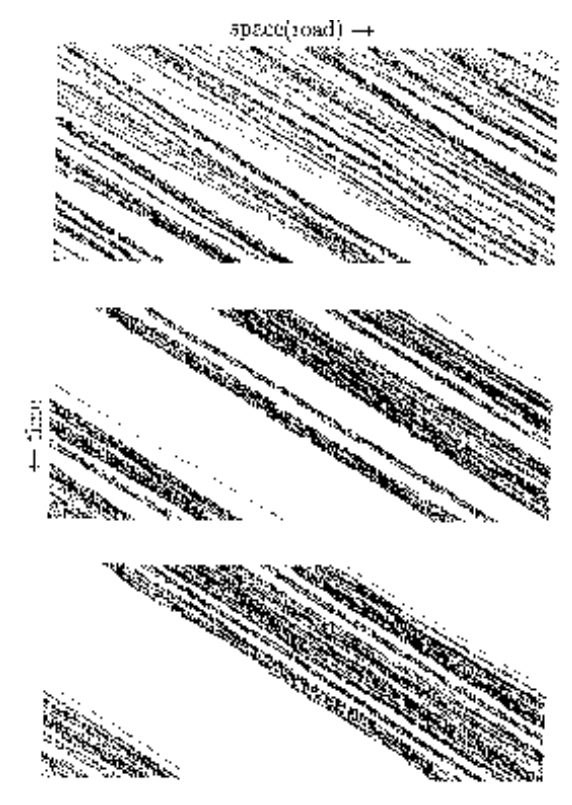

Fig. 47. Typical space-time diagram of the NaSch model with $v_{\max }=2$ and $c=0.1$ and random braking probabilities. Each horizontal row of dots represents the instantaneous positions of the vehicles moving towards right while the successive rows of dots represent the positions of the same vehicles at the successive timesteps. 
The typical size of the platoons $\xi(t)$ can be computed directly [204] by computing the correlation function (80) and identifying the separation $r=R_{0}$ of the first zero-crossing of this correlation as $\xi(t)$. Following this procedure, it has been observed that $\xi(t)$ follows the power law (111) when the distribution of the random braking probabilities is given by $P(p)=2^{n}(n+1)\left(\frac{1}{2}-p\right)^{n}$.

\subsection{Random $v_{\max }$}

The two important parameters of the NaSch model are $p$ and $v_{\max }$. In the preceding subsection we have seen the effects of randomizing $p$ assigning the same $v_{\max }$ to all the vehicles. In this subsection, on the other hand, we investigate the effects of randomizing $v_{\max }$, assigning a non-random constant $p$ to every driver.

The simplest possible model to investigate the effects of quenched randomness in $v_{\max }$ is that considered by Ben-Naim et al. [209] which was discussed to motivate the phenomenon of platoon formation in the preceding subsection.

In order to model traffic consisting of two different types of vehicles, say, for example, cars and trucks, of which a fraction $f_{\text {fast }}$ are intrinsically fast (say, cars) while the remaining fraction $1-f_{\text {fast }}$ are intrinsically slow (say, trucks), Chowdhury et al. [195] assigned a higher $v_{\max }$ (e.g., $v_{\max }=5$ ) to a fraction $f_{\text {fast }}$ of vehicles chosen randomly while the remaining fraction $1-f_{\text {fast }}$ were assigned a lower $v_{\max }$ (e.g., $v_{\max }=3$ ). As the density of the vehicles increases, the vehicles with higher $v_{\max }$ find it more difficult to change lane in order pass a vehicle with lower $v_{\max }$ ahead of it in the same lane. This leads to the formation of "coherent moving blocks" of vehicles each of which is led by a vehicle of lower $v_{\max }$ [212]. Two main causes of traffic accidents, namely, differences in vehicles speeds and lane changes, are reduced considerably in this state thereby making this state of traffic much safer. It is worth mentioning that even a small number of slow vehicles in 2-lane models, where overtaking is possible, can have a drastic effect. For details we refer to [197] and the discussion in Sec. 9.2.

\subsection{Randomly placed bottlenecks on the roads and the maximum flux prin- ciple}

So far we have investigated the effects of two different types of quenched randomness both of which were associated with the vehicles (i.e., particles). We now consider the effects of yet another type of quenched randomness which is associated with the road (i.e., lattice). 
In order to anticipate the effects of such randomness associated with the highway, let us begin with the simplest possible caricature of traffic with a "point defect" [213]: a single "impurity" (or, "defect") site in the deterministic limit $p=0$ of the NaSch model with $v_{\max }=1$. In this model, vehicles move forward, in parallel, by one lattice spacing if the corresponding site in front is empty; each vehicle takes $T_{i m p}(>1)$ timesteps to cross the "impurity" site but only one time step to cross a normal site when the next site is empty. The impurity sites acts like a blockage for all $T_{i m p}>1$. As explained in section 8.2.1, in the absence of the impurity, $J=c$ for $0<c \leq 1 / 2$ and $J=1-c$ for $1 / 2<c \leq 1$. Note that, if the impurity is present, $1 / T_{i m p}$ vehicle passes through the impurity site per unit time. Therefore, the bottleneck created by the impurity introduces an upper cut-off of the flux, viz., $1 / T_{i m p}$. Obviously, $J=c<1 / T_{i m p}$ so long as $c<c_{1}=1 / T_{i m p}$. Similarly, $J=1-c<1 / T_{i m p}$ for $c>c_{2}$ where $c_{2}=1-c_{1}$. In the density interval $c_{1}<c<1-c_{1}$, the bottleneck at the impurity is the flow-limiting factor and, hence, in this regime, $J=1 / T_{i m p}$ is independent of $c$. Thus, in the simple caricature of traffic under consideration one would expect the flux to vary with density following the relation

$$
J=\left\{\begin{array}{lll}
c & \text { if } & 0<c \leq c_{1} \\
1 / T_{i m p} & \text { if } & c_{1}<c \leq c_{2} \\
1-c & \text { if } & c_{2}<c \leq 1
\end{array}\right.
$$

where

$$
c_{1}=\frac{1}{1+(\Delta t)_{i m p}} \quad \text { and } \quad c_{2}=\frac{(\Delta t)_{i m p}}{1+(\Delta t)_{i m p}}
$$

and $T_{i m p}=1+(\Delta t)_{i m p}$ such that $(\Delta t)_{i m p}=0$ for the normal sites but $(\Delta t)_{i m p}>0$ for the impurity site.

The fundamental diagram, obtained numerically through computer simulation of the NaSch model with a single defect and non-zero $p$ (Fig. 48) is in qualitative agreement with those of the fundamental diagram (112). The qualitative features of the fundamental diagram in Fig. 48 are also similar to those of the TASEP with a single defect [214] where the hopping probability $q$ is smaller than that at all the normal sites. Equation (112) also indicates that the larger is $(\Delta t)_{i m p}$ the lower is the maximum flux $1 /\left[1+(\Delta t)_{i m p}\right]$ and the wider is the interval $c_{1} \leq c \leq c_{2}$ over which the flux remains constant.

What makes the problem of a single "point defect" nontrivial is that, over the interval $c_{1} \leq c \leq c_{2}$ of the density of the vehicles, where the $J$ is maximum and independent of $c$, the localized blockage has global effects whereby the traffic exhibits macroscopic phase segregation into high-density and low-density regions. Evidence for such macroscopic phase segregation can be obtained directly from the density profiles (see Fig. 49). Fig. 49 implies that so long as $c<c_{1}$ the particles will not pile up but a local increase of density will 


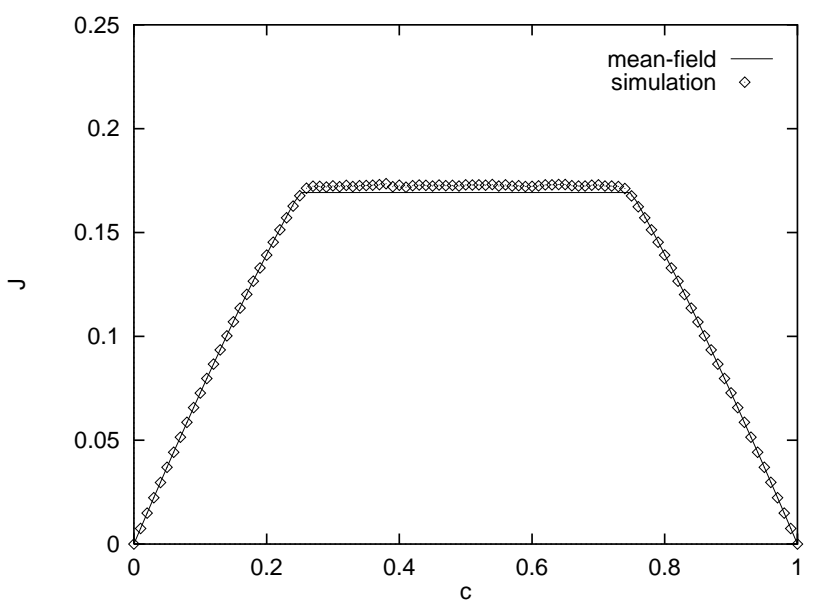

Fig. 48. Fundamental diagram of the NaSch model with $v_{\max }=1$ and a blockage site located at the site 1 . The hopping probability in the bulk is given by $q=0.75$ and at the defect by $q=0.25$.

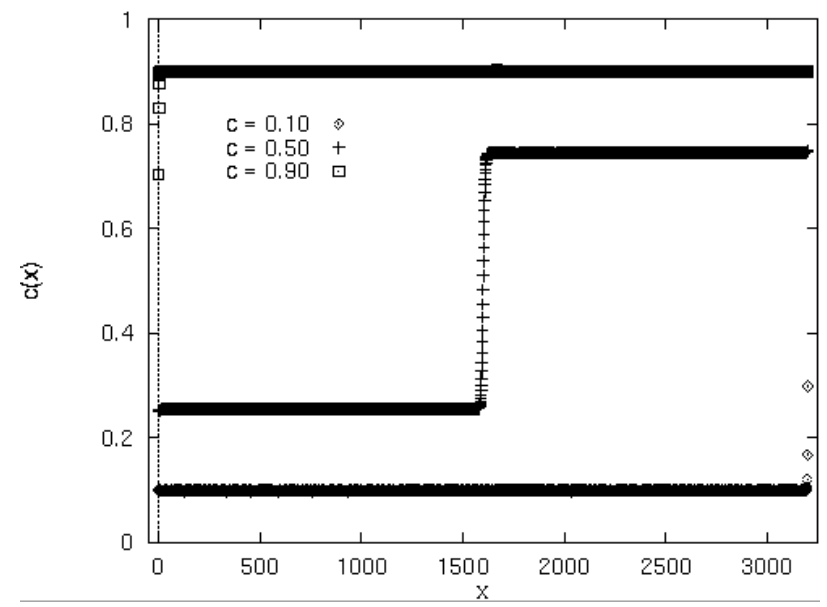

Fig. 49. Density profiles for different values of $c$ in the NaSch model with $v_{\max }=1$ and a blockage site located at the site 1 . The hopping probability in the bulk is given by $q=0.75$ and at the blockage site by $q=0.25$.

compensate for the reduced local velocity at the blockage so that the flux around the blockage is identical to that far from it. However, if the global density exceeds $c_{1}$, the particles pile up during the transient period leading to the phase-segregated steady state. Because of the particle-hole symmetry the phase-segregation does not take place if the particle density exceeds $c_{2}$.

We now develop a semi-phenomenological theory ${ }^{23}$ for the NaSch model with $v_{\max }=1$, non-zero $p$ and a single "impurity" site assuming the steady-state to be phase-segregated, as demonstrated by computer simulation (Fig. 49). Naturally, this theory cannot explain the underlying mechanism that gives rise to the phase-segregated structure of the steady-state. But, as we shall see

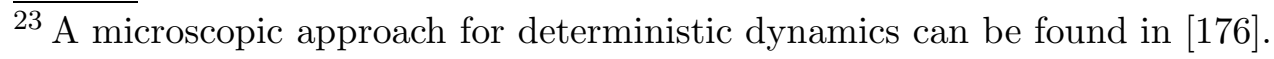


soon, it provides a good estimate of the flux in the phase-segregated regime. Our calculations are based on arguments similar to those suggested originally by Janowsky and Lebowitz [214] in the context of TASEP with a single defect.

Using equation (78), the flux in the high-density and low-density regions, far from their interface, are given by $J_{h}=\frac{1}{2}\left(1-\sqrt{1-4 q c_{h}\left(1-c_{h}\right)}\right)$ and $J_{\ell}=\frac{1}{2}\left(1-\sqrt{1-4 q c_{\ell}\left(1-c_{\ell}\right)}\right)$ and that across the defect bond is given by $J_{\text {def }} \simeq \frac{1}{2}\left(1-\sqrt{1-4 q_{d} c_{h}\left(1-c_{\ell}\right)}\right)$. Since, in the steady state, the flux is same across the entire system, we must have $q c_{h}\left(1-c_{h}\right)=q c_{\ell}\left(1-c_{\ell}\right)$ and, hence,

$$
c_{h}=c_{\ell} \quad \text { or } \quad c_{h}=1-c_{\ell}
$$

The condition $c_{h}=c_{\ell}$ is satisfied by the uniform density profiles whereas the condition $c_{h}=1-c_{\ell}$ is satisfied by the phase-segregated density profile (see Fig. 49). Moreover, using the condition $J_{h}=J_{d e f}=J_{\ell}$ we get

$$
c_{h}\left(1-c_{h}\right)=c_{\ell}\left(1-c_{\ell}\right) \simeq r c_{h}\left(1-c_{\ell}\right)
$$

where $r=q_{d} / q<1$ may be interpreted as the "transmission probability" or "permeability" of the blockage. From the (approximate) equations (115) we get

$$
c_{h} \simeq \frac{1}{r+1}=\frac{p}{p+p_{d}} \quad \text { and } \quad c_{\ell} \simeq \frac{r}{r+1}=\frac{p_{d}}{p+p_{d}}
$$

and, hence,

$$
J=\frac{1}{2}\left[1-\sqrt{1-\frac{4 q r}{(1+r)^{2}}}\right]
$$

The estimate (117) is in good agreement with the numerical data (Fig. 48) obtained from computer simulation [190]. Moreover, the estimates $c_{\ell}$ and $c_{h}$ are also in good agreement with $c_{\ell}$ and $c_{h}$, respectively, in Fig. 49.

Note that $c_{\ell}$ and $c_{h}$ depend only on $r$ and are independent of $c$. Moreover, the estimates (116) of $c_{\ell}$ and $c_{h}$ are in excellent agreement with $c_{1}$ and $c_{2}$, respectively, in Fig. 48. At first sight, these two results may appear surprising and counter-intuitive. But, we'll now show that these are related to the mechanism of the phase-segregation. Conservation of the vehicles demand that

$$
c L=c_{h} h+c_{\ell}=c_{h} h+c_{\ell}(L-h)
$$

where $h$ and $\ell=L-h$ are the lengths of the high-density and low-density regions, respectively. Thus,

$$
\frac{h}{L}=\frac{c-c_{\ell}}{c_{h}-c_{\ell}}=\frac{c(1+r)-r}{1-r}
$$


The equation (119) shows that $h / L \rightarrow 0$ as $c \rightarrow c_{\ell}$ and $h / L \rightarrow 1$ as $c \rightarrow c_{h}$. Therefore, keeping $r$ fixed as the density is increased beyond $c_{1}=c_{\ell}$, the densities of the two regions remain fixed but the high-density region grows thicker at the cost of the length of the low-density region as more and more vehicles pile up and, eventually, at $c=c_{2}=c_{h}$ the low-density region occupies a vanishingly small fraction of the total length of the system signaling the disappearance of the phase segregation. Interestingly, recasting the expressions for $c_{h}$ and $c_{\ell}$ as $c_{\ell}=1 /\left[1+(\Delta t)_{i m p}\right]$ and $c_{h}=(\Delta t)_{i m p} /\left[1+(\Delta t)_{i m p}\right]$, where $(\Delta t)_{i m p}=1 / r$, we find close formal analogies with $c_{1}$ and $c_{2}$, respectively, in equation (113) [215].

Schütz [216] considered a TASEP with sublattice-parallel update (see App. A) where the motion of the particles is deterministic (i.e., $q=1$ ) everywhere except at a defect site where they move with the probability $q_{d}<1$ (i.e., $r=q_{d}<1$ ). Exact solution is possible through a mapping on a 6 -vertex model. Later, a solution using the matrix-product Ansatz was presented in [217]. Except for minor differences, the qualitative features of the results do not differ from the corresponding approximate results obtained for $q_{d} \neq 1$ [214]. Qualitatively similar phase segregation phenomena have also been observed in a related model [202].

The qualitative features of the fundamental diagram do not change significantly if the "point-like" defect (or, impurity) is replaced by an "extended" defect [190], i.e. a few consecutive defect sites. However, with increasing length of the defect, the maximum value of the flux decreases monotonically and approaches the maximum flow of the homogeneous system where the hopping probability associated with each of the bonds is identical to that associated with the defects in our model (Fig. 50). From Figs. 50 and 51 we conclude that the monotonic decrease of the flow with increasing length of the extended defects, leads to a larger difference $c_{h}-c_{\ell}$ between the densities of the highdensity and the low-density regions of the phase-segregated steady-state.

Next, instead of a single point-like or extended "defect", let us consider the more general case of quenched "disorder" in the NaSch model with $v_{\max }=1$ where the quenched random hopping probabilities $q_{j, j+1}=1-p_{j, j+1}$ are chosen independently from some probability distribution $P(q)$, for the hopping from the cell $j$ to the cell $j+1(j=1,2, \ldots, L)$. For a given realization of the disordered system, every vehicle hopping from a given cell $i$ to the next cell $i+1$ must hop with the same probability $q_{i, i+1}$ and a given vehicle hops across different bonds, in general, with different probabilities assigned to these bonds as it moves forward with time. A similar generalization of the TASEP has also been studied [137]. We shall refer to this model as disordered TASEP (i.e., DTASEP). 


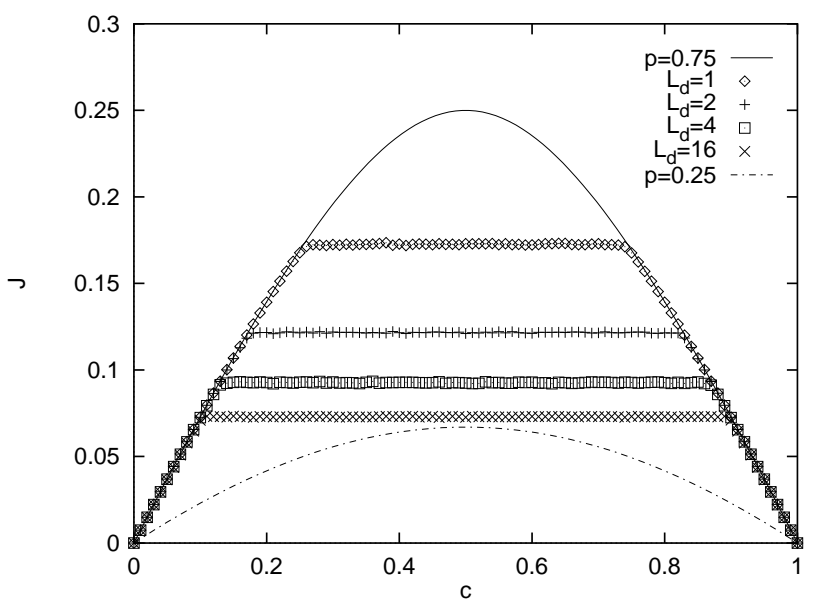

Fig. 50. Fundamental diagram for the NaSch model with $v_{\max }=1$ and defects of different length $L_{d}$. Again $p=0.75$ and $p_{d}=0.25$ is chosen. For comparison the fundamental diagram of the "fast" $(\mathrm{p}=0.75)$ and "slow" homogeneous systems are shown.

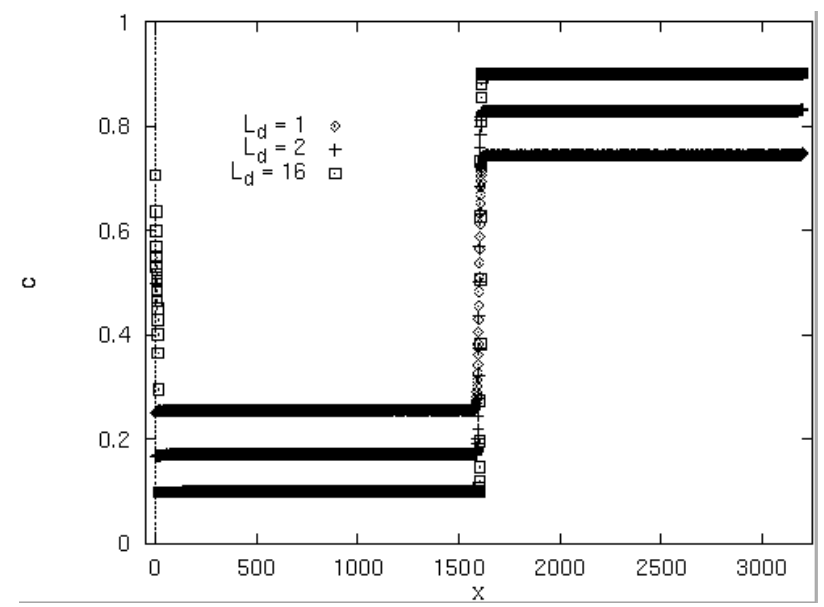

Fig. 51. Density profiles for different lengths of the defect in the NaSch model with $v_{\max }=1$. Again $p=0.75$ and $p=0.25$ are used and the average density is given by $c=0.50$. The defect extended over the first $L_{d}$ sites of the system.

Suppose, $q$ are chosen from the binary distribution

$$
P\left(q_{j, j+1}=q_{d}\right)=f, \quad P\left(q_{j, j+1}=q\right)=1-f
$$

i.e., a fraction $f$ of the bonds have a permeability $r<1$ while the remaining fraction $1-f$ have unit permeability. A mean-field theory has been developed [137] (see Appendix $\mathrm{G}$ for details) for computing the fundamental diagram of the DTASEP. The flux in this model has interesting symmetry properties under the operations of "charge conjugation" (which interchanges particles and holes), "parity" (which interchanges forward and backward hopping rates on each bond and "time reversal" (which reverses the direction of the current) $[137,218]$. 
The quenched disorder in these "disordered" models can be viewed as "pointlike impurities" distributed randomly over the lattice. But, the qualitative features of the fundamental diagram of DTASEP are similar to those observed for a single point-like defect and those for a single extended defect. Although the random distribution of the point-like impurities leads to a "rough" density profile for all densities, in an intermediate regime of density, phase-segregated steady-sates with macroscopic high- and low-density regions have been identified.

What is the underlying mechanism for the "macroscopic" phase segregation in all the models DTASEP [137] ? Let us denote the stretches of bonds with permeability 1 by $X$ and the stretches of bonds with permeability $r$ by $Y$. The two parabolas in Fig. 52 are the two steady-state fundamental diagrams for the two pure reference systems consisting of all $X$ and all $Y$, respectively. Since the flux must be spatially constant in the steady-state, the possible densities are given by the four intersections of the line $J=J_{0}$ with the two parabolas. If the average density is less (greater) than $1 / 2$ then the two possible densities are $c_{1}$ and $c_{2}\left(c_{3}\right.$ and $\left.c_{4}\right)$. The variation of density between $c_{1}$ and $c_{2}$ (or $c_{3}$ and $\left.c_{4}\right)$ in the $X$ and $Y$ stretches is merely micro phase-segregation while, on a macroscopic scale, the density remains uniform. For simplicity, we assume that the density in each stretch of like bonds is uniform. The global density of the system is approximately $c \simeq(1-f) c_{1,4}\left(J_{0}\right)+f c_{2,3}\left(J_{0}\right)$ where $f$ is given by equation (120). However, as the density increases the flux also increases till it attains the maximum allowed flux of the pure system consisting of all $Y$ (this happens at a global density smaller than $1 / 2$ ). What happens when the density increases further? According to the "maximum current principle" [162], no further increase of the flux is possible and the excess density is taken care of by increasing the density in some of the $X$ stretches from $c_{1}$ to $c_{4}$ (or, vice versa if $c>1 / 2$ ). This conversion takes place adjacent to the largest stretch of $Y$ bonds where the density also changes from $c_{2}$ to $c_{3}$ (or, vice versa if $c>1 / 2$ ) to accommodate the additional particles added to the system. This leads to the macroscopic phase segregation as the system consists of two macroscopic regions of two different mean densities- one with lower densities $c_{1}, c_{2}$ in the $X$ and $Y$ stretches and the other with the higher densities $c_{3}, c_{4}$ in the $X$ and $Y$ stretches.

It is not difficult to generalize the DTASEP to disordered NaSch (DNaSch) model by replacing the random sequential updating by parallel updating. However, we face subtle conceptual difficulties in extending DNaSch model further to arbitrary $v_{\max }$; if the position of a vehicle at time $t+1$ is decided by $q$ at its current position at time $t$, it may be forced to move $v$ sites downstream by hopping over sites of even smaller $q$ if $v>1$.

Some alternative parameterization of the defect (or, disordered) sites in the NaSch model for arbitrary $v_{\max }$ have also been suggested. In [219,190] local- 


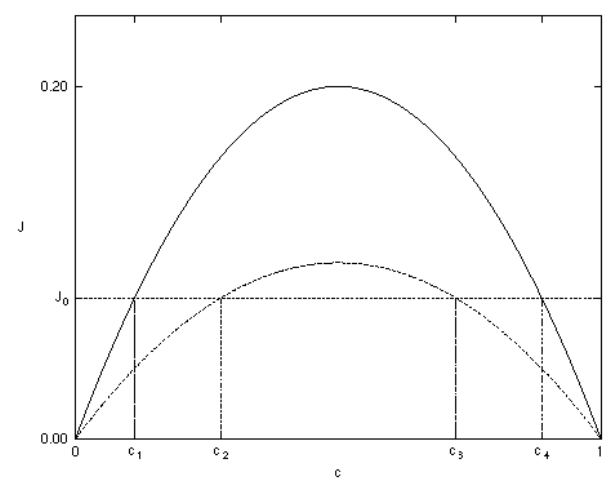

Fig. 52. Origin of phase separation in DTASEP. Shown are the fundamental diagrams of two reference non-disordered TASEPs.

ized defects have been investigated where the randomization parameter $p_{d}$ is larger than in the rest of the system. Csahok and Vicsek [220] have considered the blockages as sites with a "permeability" smaller than unity whereas the permeability of all the other sites is unity. This effectively reduces $v_{\max }$ while the vehicle is at a blockage. On the other hand, Emmerich and Rank $[221,154]$ considered a model of where the velocity of every vehicle in the region occupied by the blockage (or, more appropriately, hindrance) at the time step $t+1$ is half of that at time $t$, i.e., $v_{n}(t+1)=v_{n}(t) / 2$ is the $n$-th vehicle is located within the hindrance region. Some effects of static hindrances on vehicular traffic have also been investigated following alternative approaches, e.g., car-following theory [222]. From a practical point of view, ramps have effects very similar to those of a static defect. For the NaSch model this has been investigated in [223].

\section{Other CA models of highway traffic}

All the CA models of highway traffic described so far are basically generalizations of the minimal CA model proposed originally by Nagel and Schreckenberg [20]. We now describe a few other alternative minimal CA models and the interesting features of the corresponding results.

\subsection{Fukui-Ishibashi model}

The update rules of the Fukui and Ishibashi (FI) [120] model of single-lane highway traffic are as follows:

If $v_{\max }$ or more sites in front of the $n$-th vehicle is empty at the time step $t$, 
then it has a probability $1-p$ to move forward by $v_{\max }$ sites and a probability $p$ to move forward by $v_{\max }-1$ sites in the time step $t+1$. However, if only $d$ sites $\left(d<v_{\max }\right)$ in front of the $n$-th vehicle are empty at time $t$ then it moves by $d$ sites in the next time step. Since no site can be occupied simultaneously by more than one vehicle, a vehicle must not move forward in the time step $t+1$ if the site immediately in front of it is occupied by a vehicle at the time step $t$. The model becomes deterministic in both the limits $p=0$ and $p=1$.

The FI model differs from the NaSch model in two respects: (a) the increase of speed of the vehicles is not necessarily gradual and (b) the stochastic delay applies only to high-speed vehicles. The FI model, obviously, reduces to the NaSch model if $v_{\max }=1$. A site-oriented mean-field theory [224] and a caroriented mean-field theory $[225,226]$ for the FI model have been developed for arbitrary $v_{\max }$ and $p$. Note that the FI model is equivalent to the deterministic CA rule 184 (in the notation of Wolfram [18]) in the limit $v_{\max }=1, p=0$. Generalizations of the deterministic limit $p=0$ of the FI model have also been proposed $[227,228]$.

According to the classification of Sec. 7.3, the FI model belongs to class I, i.e. the high-acceleration limit where no spontaneous jamming exists. In [156] an alternative high-acceleration variant has been proposed. Here only the acceleration step $(U 1)$ of the NaSch model is changed to $v \rightarrow v_{\max }$, i.e. all vehicles accelerate immediately to the maximal possible velocity. The other update steps of the NaSch model are left unchanged. In contrast to the FI model, all vehicles are subject to the randomization step. The behaviour of this variant is therefore similar to that of the NaSch model, e.g. one finds spontaneous jam formation.

\subsection{Galilei-invariant vehicle-vehicle interaction and metastability}

In the NaSch model it is postulated that the gap between a pair of successive vehicles is adjusted according to the velocity of the leading vehicle alone. In contrast, often in real traffic, drivers tend to adjust the gap in front taking into account the difference between the velocity of their own vehicle and that of the leading vehicle. The latter aspect of real traffic is captured by a recent model developed by Werth, Froese and Wolf (WFW) [229,230].

If both the following vehicle and the leading vehicle move with constant acceleration $b$, then a collision between the two can be avoided provided

$$
g a p+\ell\left(v_{L V}\right) \geq v_{F V} \tau_{r}+\ell\left(v_{F V}\right)
$$

where $v_{L V}$ and $v_{F V}$ are the velocities of the leading vehicle and the following 
vehicle, respectively, $\tau_{r}$ is the reaction time of the following vehicle and

$$
\ell(v)=\frac{v^{2}}{2 b}
$$

is the distance covered by a vehicle with initial velocity $v$ before it comes to a stop by moving with a constant deceleration $b$. Using equation (122) the condition (121) for avoiding collision can be written as

$$
g a p \geq v_{F V} \tau_{r}+\frac{\bar{v}}{b}\left(v_{F V}-v_{L V}\right)
$$

where $\bar{v}=\left(v_{F V}+v_{L V}\right) / 2$ if the average velocity of the pair of vehicles under consideration. Therefore, a sufficient condition for avoiding a collision is

$$
g a p \geq \begin{cases}v_{F V} \tau_{r} & \text { for } v_{F V} \leq v_{L V} \\ v_{F V} \tau_{r}+\frac{v_{\max }}{b}\left(v_{F V}-v_{L V}\right) & \text { for } v_{F V}>v_{L V}\end{cases}
$$

In the limiting case $b \rightarrow \infty$ the sufficient condition (124) reduces to gap $\geq$ $v_{F V} \tau_{r}$ which is identical to the form of vehicle-vehicle interaction in the NaSch model if one chooses $\tau_{r}$ as the unit of time. In the opposite limit $\tau=0$, the sufficient condition (124) reduces to

$$
g a p \geq \begin{cases}0 & \text { for } v_{F V} \leq v_{L V}, \\ \frac{v_{\max }}{b}\left(v_{F V}-v_{L V}\right) & \text { for } v_{F V}>v_{L V} .\end{cases}
$$

Since this type of vehicle-vehicle interaction involves the difference of the velocities $v_{F V}-v_{L V}$, it is clearly invariant under a Galilean transformation and, hence, the name. The vehicle-vehicle interactions in real traffic may be somewhere in between the two limiting cases of NaSch model and the Galileiinvariant model.

Suppose the indices $n-1$ and $n$ label the leading vehicle and the following vehicle, respectively, of a pair. The update rules suggested by WFW [230] for implementing the Galilei-invariant vehicle-vehicle interaction are as follows:

Step 1: Acceleration.

$$
v_{n}^{(1)}=\min \left(v_{n}+1, v_{\max }\right)
$$

Step 2: Deceleration (due to other vehicles).

$$
v_{n}^{(2)}=\min \left(v_{n}^{(1)}, d_{n}-1+v_{n-1}\right)
$$

Step 3: Randomization.

$$
v_{n}^{(3)} \stackrel{p}{=} \max \left(v_{n}^{(2)}-1,0\right) \quad \text { with probability } p
$$


Step 4: Deceleration (due to other vehicles).

$$
v_{n}^{(4)}=\min \left(v_{n}^{(3)}, d_{n}-1+v_{n-1}^{(4)}\right)
$$

Step 5: Vehicle movement.

$$
\begin{aligned}
& x_{n}=x_{n}+v_{n}^{(4)}, \\
& v_{n}=v_{n}^{(4)} .
\end{aligned}
$$

Thus, the rule for deceleration (due to other vehicles) is applied twice. Step 4 makes sure collisions are avoided. Since also the new velocity $v_{n-1}^{(4)}$ of the preceding car enters, this step can not be performed in parallel for all cars. Instead it is performed sequentially, but the final configuration is independent of the starting point of this sequential updating. Step 4 has then to be applied twice in order to determine all velocities $v_{n}^{(4)}$ consistently.

The rules as given above define the retarded version of the Galilei-invariant model. In the non-retarded version, in step $2 v_{n-1}$ is replaced by the new velocity $v_{n-1}^{(2)}$. To determine $v_{n}^{(2)}$ consistently for all cars, step 2 has then to be iterated $v_{\max }-1$ times.

The most interesting feature of the Galilei-invariant model is that its fundamental diagram has a metastable branch although its update scheme involve neither cruise-control nor slow-to-start rules. The mechanism leading to the existence of metastable states is different from the models with slow-to-start rules (see Sec. 9.1.2). The outflow from jams is the same as in the NaSch model since it is independent of the interaction between vehicles. However, due to the inclusion of anticipation effects (i.e. the driver knows the velocity of the preceding vehicle) the free-flow is less sensitive to fluctuations.

\subsection{CA versions of the optimal-velocity model}

The traffic jams appear spontaneously in both the OV models and the CA models. However, in the OV models spontaneous formation of the jams are caused by the non-linearity of the dynamical equations whereas in the CA models it is triggered primarily by the stochasticity of the update "rules". The mechanism for the spontaneous formation of jams in real traffic may be a combination of these two.

In the OV model, the control of velocity is given by the control of acceleration through the OV function which gives the optimal velocity for the current distance-headway. Thus, unlike the CA models like the NaSch model, the 
vehicles in the OV models get an opportunity to avoid crash without any need to exert unphysically large deceleration. In fact, collision of vehicles may take place in naive discretization of the dynamical equations for the OV models unless special care is taken in the discretization process (see below).

In the following we present several CA model analogues of the OV model that have been proposed by different authors. In principle, the NaSch model is also an OV model, but with a linear OV function, $v(d)=\min \left[d-1, v_{\max }\right]$. The first attempt to generalize this relation is due to Emmerich and Rank [231]. The update rules of their model are as follows:

Step 1: Find largest gap.

Find the vehicle with the largest gap to the next vehicle ahead.

Step 2: Acceleration.

$$
v_{n} \rightarrow \min \left(v_{\max }, v_{n}+1\right)
$$

Step 3: Deceleration due to other vehicles.

$$
v_{n} \rightarrow M_{d_{n}-1, v_{n}} \quad \text { if } d_{n}-1 \leq v_{\max },
$$

i.e. a vehicle with velocity $j$ and $i$ empty cells in of it (i.e. a gap $d_{n}=i+1$ ) reduces its velocity to $M_{i, j}\left(0 \leq i, j \leq v_{\max }\right)$.

Step 4: Randomization.

$$
v_{n} \rightarrow \max \left(v_{n}-1,0\right) \quad \text { with probability } p
$$

Step 5: Vehicle movement.

$$
x_{n} \rightarrow x_{n}+v_{n}
$$

Step 6: Next vehicle.

Repeat steps 2-5 for the next vehicle behind, i.e. proceed in the direction opposite to the motion of the vehicles.

For the NaSch model with $v_{\max }=5$ the matrix $M_{i, j}$ is given by

$$
M_{i, j}^{(N a S c h)}=\left(\begin{array}{llllll}
0 & 0 & 0 & 0 & 0 & 0 \\
0 & 1 & 1 & 1 & 1 & 1 \\
0 & 1 & 2 & 2 & 2 & 2 \\
0 & 1 & 2 & 3 & 3 & 3 \\
0 & 1 & 2 & 3 & 4 & 4 \\
0 & 1 & 2 & 3 & 4 & 5
\end{array}\right)
$$


A general matrix $M_{i, j}$ has to satisfy certain conditions (e.g. $M_{i, j} \leq \min (i, j)$ and $M_{i, j} \leq M_{i, k}$ for $j \leq k$ ) to guarantee e.g. the absence of collisions in the model. In order to model the fact that faster vehicles keep a relatively larger headway to the preceding vehicle, Emmerich and Rank suggested the following matrix:

$$
M_{i, j}^{(E R)}=\left(\begin{array}{llllll}
0 & 0 & 0 & 0 & 0 & 0 \\
0 & 1 & 1 & 1 & 1 & 1 \\
0 & 1 & 2 & 2 & 2 & 2 \\
0 & 1 & 2 & 2 & 3 & 3 \\
0 & 1 & 2 & 3 & 3 & 4 \\
0 & 1 & 2 & 3 & 4 & 4
\end{array}\right)
$$

Using a parallel update scheme, the model shows unrealistic behaviour in the free-flow regime, especially in the deterministic limit $p \ll 1$. Here the fundamental diagram is non-monotonic [232], as can be seen from a simple example for $p=0$. At density $c=1 / 7$, the stationary state is of the form $5 \ldots \ldots .5 \ldots \ldots 5 \ldots \ldots$ where numbers denote the velocity of vehicles and '.' an empty cell. At density $c=1 / 6$, on the other hand, the stationary state is $4 \ldots . .4 \ldots . .4 \ldots .$. Comparing the corresponding flows, one finds $J(c=1 / 7)=5 / 7>J(c=1 / 6)=2 / 3$. At density $c=1 / 5$, the stationary state is $4 \ldots . \ldots . \ldots . \ldots$ with flow $J(c=1 / 5)=4 / 5$ which is again larger than the flow at $c=1 / 6$ and corresponds to the maximal possible flow. This kind of behaviour persists even in the presence of randomness $(p>0)$ [232]. In order to circumvent this problem, Emmerich and Rank had to introduce a special kind of ordered-sequential update, where first the vehicle with the largest gap ahead is updated. Then, the position of the next vehicle upstream is updated, and so on, using periodic boundary conditions.

Emmerich and Rank also investigated more general rules where even for gaps larger than $v_{\max }$ the velocity of the vehicles is reduced to a value $v<v_{\max }$.

Later, a similar model has been proposed by Helbing and Schreckenberg [233]. It is closer to the spirit of the original optimal-velocity model (see Sec. 6.2).

Step 1: Vehicle movement.

$$
x_{n} \rightarrow x_{n}+v_{n}(t)
$$

Step 2: Acceleration.

$$
v_{n}^{\prime}(t+1)=v_{n}(t)+\left\lfloor\lambda\left[V_{\text {opt }}\left(d_{n}(t)\right)-v_{n}(t)\right]\right\rfloor,
$$


Step 3: Randomization.

$$
v_{n}(t+1)=v_{n}^{\prime}(t+1)-\left\{\begin{array}{lll}
1 & \text { with probability } p & \left(\text { if } v_{n}^{\prime}(t+1)>0\right) \\
0 & \text { otherwise }
\end{array}\right.
$$

Here $\lfloor y\rfloor$ denotes the floor function, i.e. the largest integer $i \leq y$. In [233] various optimal velocity functions $V_{\text {opt }}(d)$ have been used in order to fit experimental data. The simplest, but unrealistic, choice was $V_{\text {opt }}(d)=\min \left(d, v_{\max }\right)$ where $d$ is the distance-headway. The parameter $\lambda$ corresponds formally to the sensitivity parameter in the OV model where it determines the timescale of relaxation towards the stationary fundamental diagram. Such an interpretation is not possible for discrete time models. Here the main effect of the parameter $\lambda$ is a rescaling of the OV function.

The naive discretization of the OV function produces some undesirable features of the model, e.g. the flow corresponding to the OV function is nonmonotonic in the free-flow region. Furthermore one finds - for certain initial conditions - a breakdown of the flow at a finite density $c_{*}<1$. This indicates a lack of robustness of model against small modifications of the rules.

For $\lambda<1$ the model is not intrinsically collision-free [234], in contrast to most other models discussed in this review. Problems occur e.g. when fast vehicles approach the end of a jam. For the $V_{\text {opt }}(d)=\min \left(d, v_{\max }\right)$ and the 'realistic' choice $\lambda=0.77$ [233] collisions can occur for $v_{\max } \geq 5$ [234]. For $\lambda>1$, on the other hand, a backward motion of vehicles is possible. For a given OV function it is possible to derive conditions for the parameter $\lambda$ which ensure the absence of collisions and backward motion [234].

Nagatani [235] has suggested a CA model which combines the OV idea with the TASEP. It is the discrete analogue of the simplified OV model (51) presented in Sec. 6.2. Here the $n$-th vehicle moves ahead with probability $v_{n}(t)$ where $v_{n}(t)$ is interpreted as velocity. This velocity is obtained by integration of the OV function

$$
\ddot{x}_{n}(t)= \begin{cases}a & \text { for } \Delta x_{n}(t) \geq \Delta x_{c} \\ -a & \text { for } \Delta x_{n}(t)<\Delta x_{c}\end{cases}
$$

where $a>0, \Delta x_{n}(t)=x_{n+1}(t)-x_{n}(t)$ and $\Delta x_{c}$ is a safety distance. Furthermore the velocity is restricted to the interval $0 \leq v_{n}(t) \leq v_{\max } \leq 1$. 


\subsection{CA from ultra-discretization}

Several CA which can be interpreted as traffic models have been derived using the so-called ultra-discretization method (UDM) [236]. This approach allows to establish a direct connection between certain differential equations and $\mathrm{CA}$. The problem in the derivation of CA from differential equations lies in the discretization of the 'state' (or dependent) variable. E.g. in a numerical treatment, only space and time variables are discretized.

The basic procedure of the UDM is as follows: 1) Start from a nonlinear wave equation, e.g. the KdV equation or Burgers' equation; 2) Discretize spaceand time-variables in a standard way to obtain the discrete analogue of the wave equation which is still continuous in the state variable $u_{j}(t)$; 3) The discrete analogue is now ultra-discretized. Defining $U_{j}(t)=\epsilon \ln \left(u_{j}(t)\right.$ ) (where $\epsilon$ depends on the discretization $\Delta x$ and $\Delta t$ of space and time) one can use the identity $\lim _{\epsilon \rightarrow 0} \epsilon \log \left(e^{A / \epsilon}+e^{B / \epsilon}+\cdots\right)=\max [A, B, \ldots]$ to derive the CA analogue fo the nonlinear wave equation.

By applying the UDM to Burgers' equation $v_{t}=2 v v_{x}+v_{x x}$ one obtains the $(L+1)$-state, deterministic CA $[237,238]$.

$$
n_{j}(t+1)=n_{j}(t)+\min \left[M, n_{j-1}(t), L-n_{j}(t)\right]-\min \left[M, n_{j}(t), L-n_{j+1}(t)\right]
$$

$n_{j}(t)$ is the occupation number of cell $j$ at time $t$. In contrast to most other CA discussed in this review multiple occupations of cells are allowed. The maximum number of particles which can occupy the same cell is given by $L$, i.e. $0 \leq n_{j}(t) \leq L$. The model defined by (129) might therefore by interpreted as a simple model for a highway with $L$ lanes where the effects of lane changes are completely neglected. In [238] it has also been suggested to interprete $n_{j}(t) / L$ for large $L$ as a coarse-grained density. The parameter $M$ denotes the maximum number of vehicles that can move from cell $j$ to cell $j+1$.

For $M \geq L=1$ the model reduces to the rule-184 CA, i.e. the NaSch model with $v_{\max }=1$ and $p=0$. In the case $L \leq 2 M$ the fundamental diagram looks similar to that of rule-184 CA, but it is 'degenerate' in the sense that qualitative different stationary states with the same flow exist. For $L>2 M$ the fundamental diagram the fundamental diagram resembles that of rule$184 \mathrm{CA}$ with a blockage site (see Sec. 10.3, especially Fig. 48). In the region $M / L \leq c \leq(L-M) / L$ the flow takes the constant value $M / L$, i.e. the parameter $M$ can be interpreted as a flow limiter.

In [238] also a generalization of (129) to higher velocities $v_{\max }>1$ has been suggested. For $v_{\max }=2$ the generalized update rule is given by 


$$
\begin{aligned}
n_{j}(t+1) & =n_{j}(t)+a_{j-2}(t)-a_{j}(t) \\
& +\min \left[b_{j-1}(t)-a_{j-1}(t), L-n_{j}(t)-a_{j-2}(t)\right] \\
& -\min \left[b_{j}(t)-a_{j}(t), L-n_{j+1}(t)-a_{j-1}(t)\right] .
\end{aligned}
$$

Here $b_{j}(t)=\min \left[n_{j}(t), L-n_{j+1}(t)\right]$ is the maximum number of vehicles at site $j$ that can move and $a_{j}(t)=\min \left[n_{j}(t), L-n_{j+1}(t), L-n_{j+2}(t)\right]$ is the number of vehicles that move two cells forward. The idea behind this dynamics is that first the vehicles try to move two cells forward. This is only possible if the two

cells ahead are not fully occupied, i.e. $L-n_{j+1}(t)>0$ and $L-n_{j+2}(t)>0$. Then $\min \left[b_{j}(t)-a_{j}(t), L-n_{j+1}(t)-a_{j-1}(t)\right]$ vehicles move forward one cell.

The model defined by (130) can be considered as a generalization of the FukuiIshibashi model (see Sec. 11.1) to which it reduces for $L=1$. Note that the flow limiter $M$ has been dropped in the extended model.

The fundamental diagram of the model (130) has a structure similar to that shown in Fig. 34, i.e. states of high flow exist. Due to the higher velocity the degeneracy found in the model (129) is lifted. In the case $L=2$, the $c=1 / 2$ states . . 1111 ... and . . 2020 . . are degenerate in the simple model. In the $v_{\max }=2$ case, however, in the state $\ldots 1111 \ldots$ the vehicles can increase their velocity, while in the state . . 2020 . . they can not due to the presence of the fully-occupied cells '2'. The high-flow states are unstable against local perturbations.

Another CA model obtained by ultra-discretization of the modified $\mathrm{KdV}$ equation has been suggested in [239]. It is a second-order CA since the configuration at time $t+1$ does not only depend on the configuration at time $t$, but also on the previous one at time $t-1$. The update rule for the position $x_{j}(t)$ of vehicle $j$ is given by

$x_{j}(t+2)=x_{j}(t)+\Delta x_{j}(t)+\max \left[0, \Delta x_{j}(t+1)-M\right]-\max \left[0, \Delta x_{j}(t+1)-L\right]$

where $\Delta x_{j}(t)=x_{j+1}(t)-x_{j}(t)$ is the gap and $M$ and $L$ are constants.

Although this model is deterministic it exhibits start-stop waves similar to those found in the NaSch model [239]. It seems that a second-order deterministic CA might produce effects similar to those of noise in a first-order stochastic CA. One should note, however, that the rules allow vehicles to move backwards. Other models obtained using the UDM have been discussed in $[240]$.

\section{Cellular automata models of city traffic and road networks}




\subsection{Biham-Middleton-Levine model and its generalizations}

In the BML model [21], each of the sites of a square lattice represent the crossing of a east-west street and a north-south street. All the streets parallel to the $\hat{x}$-direction of a Cartesian coordinate system are assumed to allow only single-lane east-bound traffic while all those parallel to the $\hat{y}$-direction allow only single-lane north-bound traffic. Let us represent the east-bound (northbound) vehicles by an arrow pointing towards east (north). In the initial state of the system, vehicles are randomly distributed among the streets. The states of east-bound vehicles are updated in parallel at every odd discrete time step whereas those of the north-bound vehicles are updated in parallel at every even discrete time step following a rule which is a simple extension of the TASEP: a vehicle moves forward by one lattice spacing if and only if the site in front is empty, otherwise the vehicle does not move at that time step.

Thus, the BML model is also a driven lattice gas model where each of the sites can be in one of the three possible states: either empty or occupied by an arrow $\uparrow$ or $\rightarrow$. Note that the parallel update rules of the BML model is fully deterministic and, therefore, it may also be regarded as a deterministic CA. The randomness arises in this model only from the random initial conditions [241]. Suppose, $N_{\rightarrow}$ and $N_{\uparrow}$ are the numbers of the east-bound and north-bound vehicles, respectively, in the initial state of the system. If periodic boundary conditions are imposed in all directions, the number of vehicles in every street is conserved since no turning of the vehicles are allowed by the updating rules. In a finite $L \times L$ system the densities of the east-bound and north-bound vehicles are given by $c_{\rightarrow}=N_{\rightarrow} / L^{2}$ and $c_{\uparrow}=N_{\uparrow} / L^{2}$, respectively, while the global density of the vehicles is $c=c_{\rightarrow}+c_{\uparrow}$.

Computer simulations of the BML model with periodic boundary conditions demonstrate that a first order phase transition takes place at a finite nonvanishing density $c_{*}$, where the average velocity of the vehicles vanishes discontinuously signaling complete jamming; this jamming arises from the mutual blocking ("grid-locking") of the flows of east-bound and north-bound traffic at various different crossings (see [242] for the corresponding results of the BML model with open boundary conditions).

At concentrations just above $c_{*}$, in the jammed phase, all the vehicles together form a single cluster which is stretched along the diagonal connecting the south-west to the north-east of the system. In other words, the lowestdensity jammed configurations consist of a single diagonal band where the $\rightarrow$ and $\uparrow$ occupy nearest-neighbour sites on the band in a zigzag manner. With further increase of density more and more vehicles get attached to the band in the form of off-diagonal branches and the infinite cluster of the jammed vehicles looks more and more random. Thus, in general, a typical infinite cluster of 
the jammed vehicles consists of a "backbone" and "dangling vehicles" which are the analogs of the "backbone" and the "dangling ends" of the infinite percolation clusters in the usual site/bond percolation [243]. However, in contrast to the infinite percolation cluster in the usual random site/bond percolation, the infinite spanning cluster of vehicles in the BML model emerges from the self-organization of the system. Nevertheless, concepts borrowed from percolation theory have been used to characterize the structure of the infinite cluster of jammed vehicles in the BML model at $c>c_{*}[244,245]$. The distribution of the waiting times of the vehicles at the signals (i.e., at the lattice sites) has also been investigated through computer simulations [246,247].

\subsubsection{Poor man's mean-field estimates for the BML model}

If one is not interested in detailed information on the "structure" of the dynamical phases, one can get a mean-field estimate of $c_{*}$ by carrying out a back-of-the-envelope calculation. Suppose, $c_{\rightarrow}$ and $c_{\uparrow}$ denote the average densities while $v_{\rightarrow}$ and $v_{\uparrow}$ denote the average speeds of the east-bound and northbound vehicles, respectively. In order to estimate $c_{*}$ one has to take into account interaction of the east-bound (north-bound) vehicles not only with the north-bound (east-bound) vehicles [248] but also, in a self-consistent manner, with other east-bound (north-bound) vehicles [249]. Following the arguments of Appendix I, one can show that in the symmetric case $c_{\rightarrow}=c_{\uparrow}=c$, a self-consistency equation for the speed is

$$
v_{\rightarrow}=v_{\uparrow}=v=\frac{1}{2}\left[1+\frac{c}{2}+\sqrt{\left(1+\frac{c}{2}\right)^{2}-4 c}\right]
$$

for $c<c_{*}$. The critical density $c_{*}$ is determined by the condition that at $c \geq c_{*}$ the equation (132) does not give a real solution. Hence we get $c=c_{*}=6-$ $\sqrt{32} \simeq 0.343$ which, in spite of the approximations made, is surprisingly close to the corresponding numerical estimate obtained from computer simulation [21]. Moreover, the mean-field estimate $c_{*} \simeq 0.343$ is also consistent with the more rigorous result $[251,253]$ that $c_{*}$ is strictly less than $1 / 2$. The BML model in three dimension, although not relevant for vehicular traffic, has also been studied [254].

\footnotetext{
${ }^{24}$ Ishibashi and Fukui [250] claimed that complete jamming can occur in the BML model only for $c=1$. However, a plausible flaw in their arguments was pointed out by Chau et al. [251]. In [252] it has been argued that $c_{*} \propto L^{-0.14}$, i.e. $c_{*}=0$ in the thermodynamic limit.
} 


\subsubsection{Mean-field theory of the BML model}

Recall that the occupation variables $n(i ; t)$ in the NaSch model describe the state of occupation of the sites $i(i=1,2, \ldots)$ by the vehicles on the onedimensional highway. A scheme for a truly microscopic analysis of the BML model begins [255] by introducing the corresponding generalized occupation variables $n_{\uparrow}(x, y ; t)$ and $n_{\rightarrow}(x, y ; t)$, which describe the state of occupation of the sites $(x, y)$ by the north-bound and east-bound vehicles, respectively, on the two-dimensional street-network. The analogs of the equations (64) and (65) (see Appendix I for details) have analogous physical interpretations. As usual, in the naive SOMF approximation one neglects the correlations between the occupations of different sites [255]. Since none of the sites is allowed to be occupied by more than one particle at a time, Pauli operators may be used to develop an analytical theory of vehicular traffic [256] but one should keep in mind that the particles representing the vehicles are purely classical and the system does not have any quantum mechanical characteristics.

\subsubsection{Generalizations and extensions of the BML model}

The BML model has been generalized and extended to take into account several realistic features of traffic in cities.

\section{- Asymmetric distribution of the vehicles:}

Suppose the vehicles are distributed asymmetrically among the east-bound and north-bound streets [248], i.e., $c_{\rightarrow} \neq c_{\uparrow}$. For convenience, let us write $c_{\uparrow}=$ $c f_{a}$ and $c_{\rightarrow}=c\left(1-f_{a}\right)$ where $f_{a}$ is the fraction of the vehicles moving towards north. Clearly $f_{a}=1 / 2$ corresponds to the symmetric case $c_{\rightarrow}=c_{\uparrow}=c / 2$. On the other hand, $f_{a}=0\left(f_{a}=1\right)$ correspond to the extreme asymmetric case where all the vehicles are east-bound (north-bound). Obviously, the absence of grid-locking in the extreme limits $f_{a}=0$ and $f_{a}=1$ rules out the possibility of BML-like complete jamming transition, i.e., $c_{*}=1$ for both $f_{a}=0$ and $f_{a}=1$. Moreover, $c_{*}$ decreases with decreasing asymmetry in the distribution of the vehicles; $c_{*}$ is the smallest for $f_{a}=1 / 2$, i.e., symmetric distribution of the vehicles. These results can be presented graphically by plotting the curve $c_{*}\left(f_{a}\right)$ in the phase diagram on the $c-f_{a}$ plane [248].

\section{- Unequal maximum velocities:}

In the BML model both east-bound and north-bound vehicles can move by a maximum of one lattice spacing at a time and, therefore, the average speeds of both types of vehicles can never exceed unity. On the other hand, recall that in the Fukui-Ishibashi model [120] of highway traffic vehicles can move up to a maximum of $M$ lattice sites at a time and, hence, have average velocities larger than unity. Incorporating similar high-speed vehicles Fukui et al. [257] 
generalized the BML model where the east-bound vehicles are allowed tom ove by $M$ sites at a single time step while the north-bound vehicles can move by only one lattice spacing.

\section{- Overpasses or two-level crossings:}

The BML model has been extended to take into account the effects of overpasses (or two-level crossings) [258]. A fraction $f_{o}$ of the lattice sites in the BML model are randomly identified as overpasses each of which can accommodate up to a maximum of two vehicles simultaneously. The overpasses weaken the grid-locking in the BML model. Therefore, $c_{*}$ is expected to increase with increasing $f_{o}$. Besides, $c_{*}$ is expected to be unity if $f_{o}=1$. Naturally, we address the question: does jamming disappear (i.e., $c_{*}$ is unity) only at $f_{o}=1$ or for even smaller values of $f_{o}$ ? In order to answer this question we extend the self-consistent mean-field arguments, which led to the equation (132), incorporating the effects of the overpasses thereby getting the generalized selfconsistency equation [259]

$$
v_{\rightarrow}=v_{\uparrow}=v=\frac{1}{2}\left[1+\frac{1-f_{o}}{2} c+\sqrt{\left(1+\frac{1-f_{o}}{2} c\right)^{2}-4\left(1-f_{o}\right) c}\right]
$$

in the symmetric case $c_{\rightarrow}=c_{\uparrow}=c$. The equation (133) reduces to the equation (132) in the limit $f_{o}=0$. It predicts that, if $f_{o} \neq 0$, a moving phase exists in the BML model with overpasses for vehicle densities $c \leq c_{*}=(6-\sqrt{32}) /\left(1-f_{o}\right)$ and that the jammed phase disappears altogether (i.e., $c_{*}$ becomes unity) for $f_{o} \geq 1-0.343=0.657$, an underestimate when compared with the corresponding computer simulations. These results can be presented graphically by drawing the curve $c_{*}\left(f_{o}\right)$ in the mean-field phase diagram on the $c-f_{o}$ plane not only for the symmetric distribution (i.e., for $f=1 / 2$ ) but also for asymmetric distributions of the vehicles among the east-bound and north-bound streets [259].

\section{- Faulty traffic lights:}

The effects of faulty traffic lights have been modeled by generalizing the BML as follows [260]: a fraction $f_{t l}$ of the sites are identified randomly as the locations of the faulty traffic lights. Both the north-bound vehicles currently south of the faulty traffic lights and the east-bound vehicles currently west of a faulty traffic light are allowed to hop onto the empty crossings where the faulty traffic lights are located, irrespective of whether the corresponding time step of updating is odd or even. If an east-bound vehicle and a north-bound vehicle simultaneously attempt to enter the same crossing, where the faulty traffic light is located, then only one of then is allowed to enter that crossing by selecting randomly. 
Since a north-bound (east-bound) vehicle will be able to move forward although only east-bound (north-bound) vehicles would have moved forward had $f_{t l}$ been zero, the average velocity of the vehicles is expected to increase with increasing $f_{t l}$. However, with the increase of $f_{t l}$ there is an increasing likelihood that an east-bound (north-bound) vehicle would be blocked by a north-bound (east-bound) vehicle at a faulty traffic light. Thus, the increasing density of faulty traffic lights increases the effect of grid-locking thereby decreasing $c_{*}$.

\section{- Static hindrances:}

The BML model has been extended to incorporate the effects of static hindrances or road blocks (e.g., vehicles crashed in traffic accident), i.e., stagnant points [261,262]. A vehicle, which stays at a normal site for only one time step before attempting to move out of it, stays at a point-like blockage for $T_{p}$ time steps before attempting to move out of it. Obviously, the longer is $T_{p}$, the lower is the corresponding $c_{*}$. The time-dependent phenomenon of spreading of the jam from the blockage site during the approach of the system to its jammed steady-state configurations has also been investigated $[261,263]$.

\section{- Stagnant street:}

Let us consider the effects of a stagnant street, where the local density $c_{s}$ of the vehicles is initially higher than that in the other streets [264], on the traffic flow in the BML model. The stagnant street, effectively, acts like a "line-defect", rather than a "point-defect". However, in contrast to the static roadblocks, a stagnant street offers a time-dependent hindrance to the vehicles moving in the perpendicular streets. As intuitively expected, the jamming transition has been found to occur at a lower global density when the local density in the stagnant street is higher.

\section{- Independent turning of the vehicles:}

Let us now generalize the BML model by assigning a trend or preferred direction of motion, $W_{n}(x, y)$, to each vehicle $n(n=1,2, \ldots)$ located at the site $x, y$. According to this definition, the vehicle $n$, located at $x, y$ jumps to the next site towards east with probability $W_{n}(x, y)$ while $1-W_{n}(x, y)$ is the corresponding probability that it hops to the next site towards north [265]. In this generalized model vehicles can take a turn but the processes of turning from east-bound (north-bound) to north-bound (east-bound) streets is stochastic. For simplicity, suppose, $N / 2$ vehicles are assigned $W_{n}(x, y)=\gamma$ while the remaining $N / 2$ vehicles are assigned $W_{n}(x, y)=1-\gamma$ where $0 \leq \gamma \leq 1 / 2$; this implies that $N / 2$ vehicles move preferentially east-ward whereas the remaining $N / 2$ vehicles move preferentially towards north. In the limit $\gamma=0$ no vehicle can turn and we recover the original BML model with deterministic update rules. The most dramatic effect of the stochastic turning is that the 
discontinuous jump $\Delta\langle v\rangle$ of the average velocity $\langle v\rangle$ decreases with increasing $\gamma$ and, eventually, the first order jamming transition ends at a critical point where $\Delta\langle v\rangle$ just vanishes.

\section{- Jam-avoiding turn and drive:}

In the model of turning considered in references [265] a vehicle turns stochastically independently of the other vehicles. In real traffic, however, a vehicle is likely to turn if its forward movement is blocked by other vehicles ahead of it in the same street. Therefore, let us now consider a model [266] where an east-bound (north-bound) vehicle turns north (east) with probability $p_{t u r n}$ if blocked by another vehicle in front of it. Computer simulations of this model shows that $c_{*}\left(p_{\text {turn }}\right)$ increases with increasing $p_{\text {turn }}$. In a slightly different model [267], on being blocked by a vehicle in front, an east-bound (north-bound) vehicle hops with probability $p_{j a}$ to the next east-bound (north-bound) street towards north (east).

\section{- A single north-bound street cutting across east-bound streets}

Let us now consider a special situation where only one north-bound street exists which cuts all of the $L$ equispaced mutually parallel east-bound streets of length $L$ [268]. This situation can be modeled as a $L \times L$ square lattice and each cell on the north-bound street can be in one of the three allowed states, namely, either empty or occupied by a $\rightarrow$ or a $\uparrow$. But, in contrast to the BML model, there are two allowed states for each cell (outside the crossings) on the east-bound street; these can be either empty or occupied by only $\rightarrow$. Since no grid-locking is possible with only one north-bound street, complete jamming occurs trivially in this case only if each of the cells either on the eastbound streets or on the north-bound street or on all the streets are occupied simultaneously by vehicles. Nevertheless, at any finite nonvanishing density, the crossings of the east-bound and north-bound streets act like hindrances with finite non-vanishing permeability for the flow of the east-bound traffic. Obviously, the higher is the density of the north-bound vehicles, the lower is the permeability and the stronger is the rate-limiting factor of the bottleneck. A comparison of this problem with that of highway traffic in the presence of static hindrances $[214,137]$ explains not only why the flux along the east-bound streets exhibits a flat plateau over an intermediate density regime but also why the plateau appears at lower values of the flux with increasing density of the north-bound vehicles [268]. A mean-field theory for the macroscopic phase segregation in this model has been developed by appropriately modifying that for the similar phenomenon in the one-dimensional models of highway traffic in the presence of static hindrances. It is worth emphasizing here that in [268] each site on the east-bound streets has been interpreted as a cell, which can accommodate one vehicle at a time, rather than as a crossing of the eastbound street with a north-bound street. Conceptually, this is an extension of 
the BML model. Finally, we briefly mention that in [269] the phase diagram of a system consisting of one east-bound and one north-bound street with one crossing has been investigated.

\section{- Green-wave synchronization}

\begin{tabular}{|c|c|c|}
\hline BML Model & Green Wave Model & Time \\
\hline$\underset{\uparrow}{\rightarrow} \rightarrow \longrightarrow \sqcup \sqcup$ & $\underset{\uparrow}{\uparrow} \rightarrow \rightarrow \sqcup \sqcup \sqcup$ & 0 \\
\hline$\rightarrow \underset{\uparrow}{\rightarrow} \sqcup \rightarrow \sqcup \sqcup$ & $\underset{\uparrow}{\uparrow} \rightarrow>\rightarrow \sqcup \sqcup$ & 2 \\
\hline$\rightarrow \underset{\uparrow}{\sqcup} \rightarrow \sqcup \rightarrow \sqcup$ & $\underset{\uparrow}{\sqcup} \rightarrow \rightarrow \rightarrow \sqcup$ & 4 \\
\hline $\begin{aligned} \rightarrow & \uparrow \rightarrow \sqcup \rightarrow \sqcup \\
\sqcup & \llcorner\end{aligned}$ & 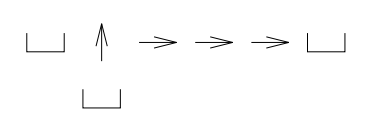 & 5 \\
\hline$\rightarrow \uparrow \sqcup \rightarrow \sqcup \rightarrow$ & $\sqcup \uparrow \sqcup \rightarrow \rightarrow \rightarrow$ & 6 \\
\hline
\end{tabular}

Fig. 53. Comparison of the update procedure in the BML model (left) and the GW model (right) (from [270]).

Often the traffic lights along the main streets in cities are synchronized to allow continuous flow; this is usually referred to as "green-wave" synchronization. A green-wave (GW) model has been developed [270] by replacing the parallel updating scheme of the BML model by an updating scheme which is partly backward-ordered sequential (see Appendix A for a general explanation of this update scheme). At odd time steps, an east-bound vehicle moves by one lattice spacing if the target site was empty at the end of the previous time step or has become empty in the current time step (this is possible because of the backward-ordered sequential updating at every time-step). Similarly the positions of the north-bound vehicles are updated at every even time step. The main difference between the BML model and the GW model (see Fig. 53), arising from the different updating schemes, is that in the GW model vehicles move as "convoys" (a cluster of vehicles with no empty cell between them) thereby mimicking the effects of green-wave synchronization of the traffic lights in real traffic. The jamming transition in the GW model has been investigated by a combination of a mean-field argument and numerical input from computer simulations [270].

\section{- More realistic description of streets and junctions}


At first sight the BML model may appear very unrealistic because the vehicles seem to hop from one crossing to the next. However, it may not appear so unrealistic if each unit of discrete time interval in the BML model is interpreted as the time for which the traffic lights remain green (or red) before switching red (or green) simultaneously in a synchronized manner, and over that time scale each vehicle, which faces a green signal, gets an opportunity to move from $j$-th crossing to the $(j+1)$-th (or, more generally [257], to the $(j+r)$-th where $r>1$ ).

In the original version of the BML model the vehicles are located on the lattice sites which are identified as the crossings. Brunnet and Goncalves [271] considered a modified version where, instead, the vehicles are located on the bonds and, therefore, never block the flow of vehicles in the transverse direction. Consequently, in this version of the CA model of city traffic jams of only finite sizes can form and these jams have finite lifetime after which they disappear while new jams may appear elsewhere in the system; an infinitely long-lived jam spanning the entire system is possible only in the trivial limit $c=1$. In contrast, Horiguchi and Sakakibara [272] generalized the BML by replacing each of the bonds connecting the nearest-neighbour lattice sites by a bond decorated with an extra lattice site in between. In [273] a generalization to $s$ extra lattice sites between crossings has been presented. However, the vehicles are still allowed to hop forward by only one lattice spacing in the model of Horiguchi and Sakakibara. Moreover, generalizing the rules for turning of the vehicles in Ref. [265], Horiguchi and Sakakibara also allowed probabilistic turning of the vehicles in their model. The model exhibits a transition from the flowing phase to a completely jammed phase.

The streets in the original BML model were assumed to allow only one-way traffic. This restriction was relaxed in a more realistic model proposed by Freund and Pöschel [274] which allows both-way traffic on all the streets. Thus, each east-west (north-south) street is implicitly assumed to consist of two lanes one of which allows east-bound (north-bound) traffic while the other allows west-bound (south-bound) traffic. Moreover, each site is assumed to represent a crossing of a east-west street and a north-south street where four numbers associated with the site denote the number of vehicles coming from the four nearest-neighbour crossings (i.e., from north, south, east and west) and queued up at the crossing under consideration. So, in this extended version of the BML model, each site can accommodate at most $4 Q$ particles if each of the four queues of vehicles associated with it is allowed to grow to a maximum length $Q$.

In the model proposed by Freund and Pöschel [274], initially, each of the vehicles is assigned a site selected at random, the queue to which it belongs (i.e., whether it is approaching the crossing from north,south, east or west) and the desired direction (i.e., left, right or straight) for its intended motion 
at the next time step. At each discrete time step a vehicle is allowed to move forward in its desired direction of motion by one lattice spacing provided (a) it is at the front of the queue in its present location and (b) there are fewer than $Q$ vehicles queued up at the next crossing in the same desired direction of motion. Once a vehicle moves to the next crossing it finds a place at the end of the corresponding queue at the new crossing while the vehicles in the queue it left behind are moved "closer to the crossing" by one position (by mere relabeling as no physical movement of the vehicles in the queue takes place explicitly). Various reasonable choices for the rule which determines the desired direction of each vehicle at every time step have also been considered.

The finite space of the streets in between successive crossings do not appear explicitly in the extension of the BML model suggested by Freund and Pöschel [274] although it is more realistic than the BML model because it implicitly takes into account the possibility of formation of queues by vehicles approaching one crossing from another. Chopard et al. [275] have developed a more realistic CA model of city traffic where the stretches of the streets in between successive crossings appear explicitly. In this model also each of the streets consist of two lanes which allow oppositely directed traffic. The rule for implementing the motion of the vehicles at the crossing is formulated assuming a rotary to be located at each crossing. Depending on the details of the rules to be followed by the vehicles at the rotary, the system can exhibit a variety of phenomena. For example, the flow can be metastable at all densities if each of the vehicles on the rotary is required to stop till the destination cell becomes available for occupation [275]. Moreover, the bottleneck created by the vehicles on the rotaries at the junctions can lead to a plateau in the fundamental diagram which is analogous to that caused by a static hindrances on a highway $[214,137]$.

\subsection{Marriage of the NaSch model and the BML model; a "unified" CA model of city traffic}

If one wants to develop a more detailed "fine-grained" description of city traffic than that provided by the BML model then one must first decorate each bond [268,272] with $D-1(D>1)$ sites to represent $D-1$ cells in between each pair of successive crossings $[268,275]$ thereby modeling each segment of the streets in between successive crossings in the same manner in which the entire highway is modelled in the NaSch model. Then, one can follow the prescriptions of the NaSch model for describing the positions, speeds and accelerations of the vehicles $[275,276]$ as well as for taking into account the interactions among the vehicles moving along the same street. Moreover, one should flip the color of the signal periodically at regular interval of $T(T \gg 1)$ time steps where, during each unit of the discrete time interval every vehicle facing green signal 
should get an opportunity to move forward from one cell to the next. Such a CA model of traffic in cities has, indeed, been proposed very recently $[277,278]$ where the rules of updating have been formulated in such a way that, (a) a vehicle approaching a crossing can keep moving, even when the signal is red, until it reaches a site immediately in front of which there is either a halting vehicle or a crossing; and (b) no grid-locking would occur in the absence of random braking.

Let us model the network of the streets as a $N \times N$ square lattice. For simplicity, let us assume that the streets parallel to $\hat{x}$ and $\hat{y}$ axes allow only single-lane east-bound and north-bound traffic, respectively, as in the original formulation of the BML model. Next, we install a signal at every site of this $N \times N$ square lattice where each of the sites represents a crossing of two mutually perpendicular streets. We assume that the separation between any two successive crossings on every street consists of $D$ cells so that the total number of cells on every street is $L=N \times D$. Each of these cells can be either empty or occupied by at most one single vehicle at a time. Because of these cells, the network of the streets can be viewed as a decorated lattice. However, unlike the BML model [21], which corresponds to $D=1$, and the model of Horiguchi and Sakakibara [272], which corresponds to $D=2, D(<L)$ in this model is to be treated as a parameter. Note that $D$ introduces a new length scale into the problem.

The signals are synchronized in such a way that all the signals remain green for the east-bound vehicles (and simultaneously, red for the north-bound vehicles) for a time interval $T$ and then, simultaneously, all the signals turn red for the east-bound vehicles (and, green for the north-bound vehicles) for the next $T$ timesteps. Clearly, the parameter $T$ introduces a new time scale into the problem. Thus, in contrast to the BML model, the forward movement of the individual vehicles over smaller distances during shorter time intervals are described explicitly in this "unified" model.

If no turning of the vehicles is allowed, as in the original BML model, the total number of vehicles on each street is determined by the initial condition, and does not change with time because of the periodic boundary conditions. Following the prescription of the NaSch model, we allow the speed $v$ of each vehicle to take one of the $v_{\max }+1$ integer values $v=0,1, \ldots, v_{\max }$. Suppose, $v_{n}$ is the speed of the $n$-th vehicle at time $t$ while moving either towards east or towards north. At each discrete time step $t \rightarrow t+1$, the arrangement of $N$ vehicles is updated in parallel according to the following "rules":

Step 1: Acceleration.

$v_{n} \rightarrow \min \left(v_{n}+1, v_{\max }\right)$.

Step 2: Deceleration (due to other vehicles or signal). 


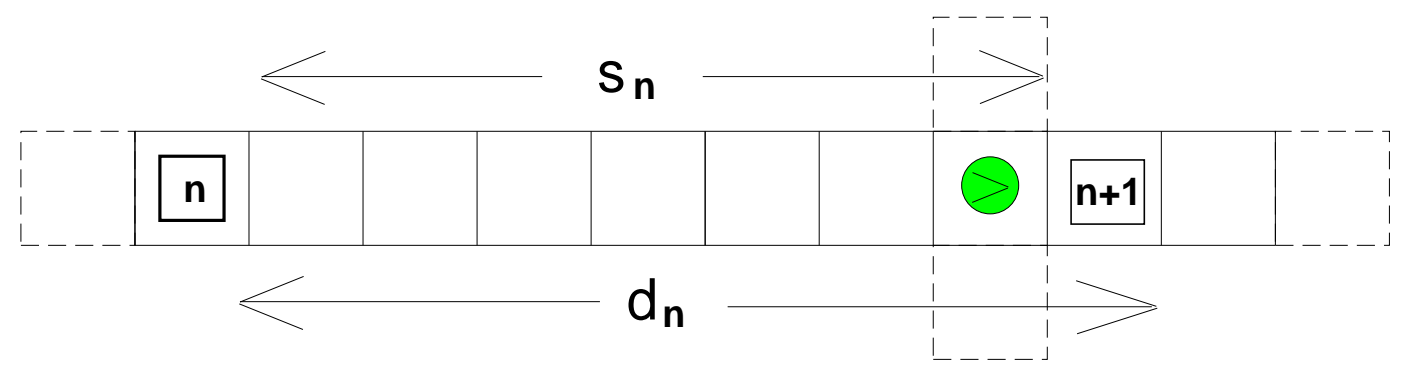

Fig. 54. A segment of a east-west street, where the $n$-th and the $(n+1)$-th vehicles are located, is shown schematically together with one of the nearest signals.

Suppose, $d_{n}$ is the gap in between the $n$-th vehicle and the vehicle in front of it, and $s_{n}$ is the distance between the same $n$-th vehicle and the closest signal in front of it (see Fig. 54).

Case I: The signal is red for the $n$-th vehicle under consideration:

$v_{n} \rightarrow \min \left(v_{n}, d_{n}-1, s_{n}-1\right)$.

Case II: The signal is green for the $n$-th vehicle under consideration:

Suppose, $\tau$ is the number of the remaining time steps before the signal turns red. Now there are two possibilities in this case:

(i) When $d_{n} \leq s_{n}$, then $v_{n} \rightarrow \min \left(v_{n}, d_{n}-1\right)$. The motivation for this choice comes from the fact that, when $d_{n} \leq s_{n}$, the hindrance effect comes from the leading vehicle.

(ii) When $d_{n}>s_{n}$, then $v_{n} \rightarrow \min \left(v_{n}, d_{n}-1\right)$ if $\min \left(v_{n}, d_{n}-1\right) \times \tau>s_{n}$; else $v_{n} \rightarrow \min \left(v_{n}, s_{n}-1\right)$. The motivation for this choice comes from the fact that, when $d_{n}>s_{n}$, the speed of the $n$-th vehicle at the next time step depends on whether or not the vehicle can cross the crossing in front before the signal for it turns red.

\section{Step 3: Randomization.}

$v_{n} \rightarrow \max \left(v_{n}-1,0\right)$ with probability $p(0 \leq p \leq 1) ; p$, the random deceleration probability, is identical for all the vehicles and does not change during the updating.

\section{Step 4: Vehicle movement.}

For the east-bound vehicles, $x_{n} \rightarrow x_{n}+v_{n}$ while for the north-bound vehicles, $y_{n} \rightarrow y_{n}+v_{n}$.

The rule in case II of step 2 can be simplified without changing the overall behaviour of the model [278]:

Case II':

If the signal turns to red in the next timestep $(\tau=1)$ :

$v_{n} \rightarrow \min \left(v_{n}, s_{n}-1, d_{n}-1\right)$

else

$v_{n} \rightarrow \min \left(v_{n}, d_{n}-1\right)$. 
These rules are not merely a combination of the rules proposed by Biham et al. [21] and those introduced by Nagel and Schreckenberg [20] but also involve some modifications. For example, unlike all the earlier BML-type models, a vehicle approaching a crossing can keep moving, even when the signal is red, until it reaches a site immediately in front of which there is either a halting vehicle or a crossing. Moreover, if $p=0$ every east-bound (north-bound) vehicle can adjust speed in the deceleration stage so as not to block the northbound (east-bound) traffic when the signal is red for the east-bound (northbound) vehicles.

Initially, we put $N_{\rightarrow}$ and $N_{\uparrow}$ vehicles at random positions on the east-bound and north-bound streets, respectively. We update the positions and velocities of the vehicles in parallel following the rules mentioned above. After the initial transients die down, at every time step $t$, we compute the average velocities $\left\langle v_{x}(t)\right\rangle$ and $\left\langle v_{y}(t)\right\rangle$ of the east-bound and north-bound vehicles, respectively.

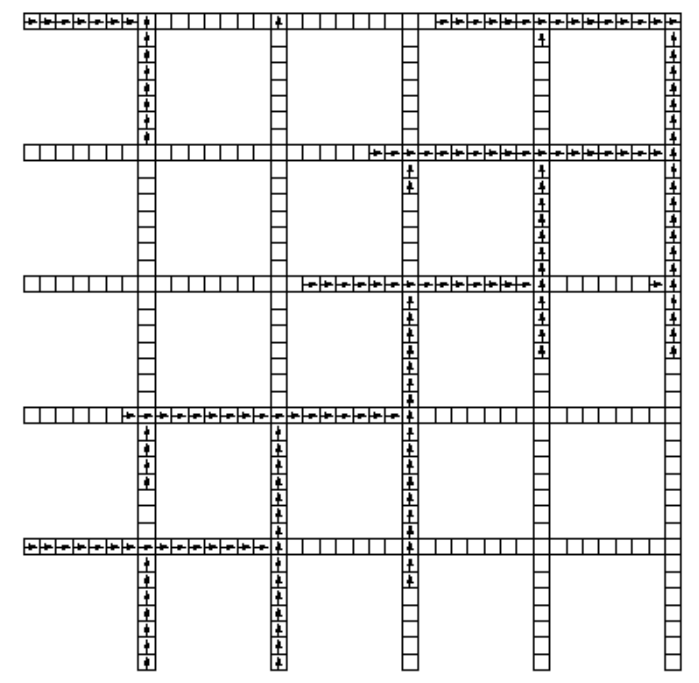

Fig. 55. A typical jammed configuration of the vehicles $(N=5, D=8)$. The east-bound and north-bound vehicles are represented by the symbols $\rightarrow$ and $\uparrow$, respectively.

A phase transition from the "free-flowing" dynamical phase to the completely "jammed" phase takes place in this model at a vehicle density $c_{*}(D)$. The dependence on the dynamical parameters $p, v_{\max }$ and $T$ is not clear yet [278]. The data obtained so far from computer simulations do not conclusively rule out the possibility that the transition density only depends on the structure of the underlying lattice, similar to the percolation transition [243], and is independent of the dynamical parameters. The intrinsic stochasticity of the dynamics, which triggers the onset of jamming, is similar to that in the NaSch model, while the phenomenon of complete jamming through self-organization as well as the final jammed configurations (see Fig. 55) are similar to those in the BML model. The variations of $\left\langle v_{x}\right\rangle$ and $\left\langle v_{y}\right\rangle$ with time as well as with $c$, $D, T$ and $p$ in the flowing phase are certainly more realistic that in the BML 
model [277].

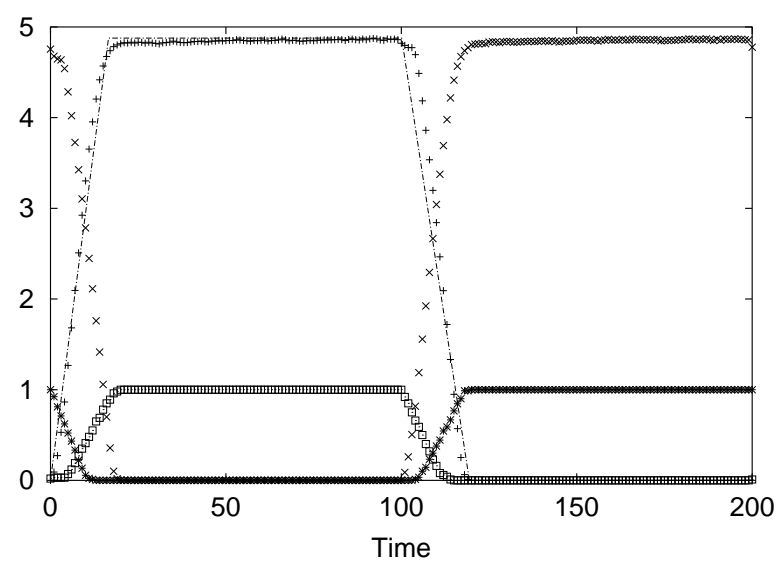

Fig. 56. Time-dependence of average speeds of vehicles. The symbols,$+ \times, *$ and $\square$ correspond, respectively, to $\left\langle v_{x}\right\rangle,\left\langle v_{y}\right\rangle$, and the fractions of vehicles with instantaneous speed $v=0, f_{x 0}$ and $f_{y 0}$, respectively. The common parameters are $v_{\max }=5, p=0.1, D=100, T=100$ and $c=0.1$. The continuous line has been obtained from heuristic arguments given in [277].

The "unified" model has been formulated intentionally to keep it as simple as possible and at the same time capture some of interesting features of the NaSch model as well as the BML model. We believe that this model can be generalized (i) to allow traffic flow both ways on each street which may consist of more than one lane, (ii) to make more realistic rules for the right-of-the-way at the crossings and turning of the vehicles, (iii) to implement different types of synchronization or staggering of traffic lights [276] (including green-wave), etc.

12.3 Practical applications of the models of vehicular traffic; On-line simulation of traffic networks

A large fraction of the available resources are spent by the governments, particularly in the industrialized developed countries, to construct more highways and other infrastructural facilities related to transportation. The car-following models, the coupled-map lattice models as well as the CA models have been used for computer simulation with a hope to utilize the results for on-line traffic control. For planning and design of the transportation network [19], for example, in a metropolitan area [279-281], one needs much more than just micro-simulation of how vehicles move on an idealized linear or square lattice under a specified set of vehicle-vehicle and road-vehicle interactions. For such a simulation, to begin with, one needs to specify the roads (including the number of lanes, ramps, bottlenecks, etc.) and their intersections. Then, times and places of the activities, e.g., working, shopping, etc., of individual 
drivers are planned. Micro-simulations are carried out for all possible different routes to execute these plans; the results give informations on the efficiency of the different routes and these informations are utilized in the designing of the transportation network [19]. Some socio-economic questions as well as questions on the environmental impacts of the planned transportation infrastructure also need to be addressed during such planning and design. For a thorough discussion of these aspects we refer to the recent review by Nagel et al. [19].

\section{Some related systems, models and phenomena}

In this section we will briefly present some stochastic models and phenomena which are somehow related to the main topic of this review. For some models the relation to traffic flow is rather obvious, e.g. there are toy models which share some features with traffic models, but which can be solved exactly. We also point out similarities in the description of other phenomena, e.g. granular flow or surface growth. Similarities also exist to systems from solid state physics, namely ionic conductors. This point will not discussed here, instead we refer to refs. [282,283]. Finally we like to mention that there are some resemblances between the CA models of vehicular traffic and the CA models of driven diffusive Frenkel-Kontorova-type systems [284].

\subsection{Generalizations of the TASEP}

The TASEP is probably one of the best studied models in nonequilibrium physics. Using powerful methods like MPA or Bethe Ansatz recently it became possible to calculate not only simple expectation values in the stationary states, but also more complicated quantities like diffusion constants or the large-deviation function. For a more detailed discussion and a list of references we refer to the recent reviews on the TASEP [22,174] (see also [15]). Several variants of the TASEP have been proposed. Most of those preserve the exact solvability. In the following we will discuss briefly some of the variants and generalizations of the TASEP discussed so far in the literature.

\section{- Partially asymmetric exclusion process}

An obvious generalization of the TASEP is to allow hopping processes in both directions [285]. Here only results for the random-sequential update exist, since for parallel dynamics ambiguities in the updating appear when two particles attempt to hop onto the same site. One finds that the phase diagram looks essentially like that of the TASEP with three different phases (see Sec. 8.5). Recently it has been shown [286], however, that the high- and low- 
density phases can be divided into three subphases (AI, AII, AIII and BI, BII, BIII) instead of two in the TASEP case. Again the phase boundaries of these subphases are determined by the behaviour of the density profiles and the corresponding correlation lengths.

\section{- Multispecies models I}

Karimipour and collaborators [287] have developed a multispecies generalization of the TASEP which retains the solvability by MPA. It is similar to the disordered model discussed in Sec. 10.1 where each particle is characterized by a hopping rate (also called "velocity" in this context) $v_{j}\left(j=1, \ldots, N_{s}\right)$, i.e. there are $N_{s}$ different "species" of particles. In contrast to the models with quenched disorder discussed in Sec. 10, however, overtaking of particles is possible, i.e. the ordering of the particles is not fixed.

More specifically the dynamics of the model is defined as follows: A particle is chosen at random (random-sequential update). If the particle is of species $j$ and the cell to its right is empty, it hops there with rate $v_{j}$. If the cell is occupied by a particle of species $l$ and $v_{l}<v_{j}$, then they interchange there positions with rate $v_{j}-v_{l}$. This means that a fast particle can overtake a slower one with a rate proportional to their velocity difference.

\section{- Multispecies models II}

In several papers multispecies generalizations of the ASEP have been suggested which exhibit phase separation and spontaneous symmetry breaking.

Arndt et al. [288] considered a system of positive and negative charged particles diffusing on a ring in opposite directions. Positive particles move to an empty right neighbour and negative particles move to an empty left neighbour with the same rate $\lambda$. Furthermore positive and negative particles on neighbouring sites can exchange their positions. The process $-+\rightarrow+-$ occurs with rate 1 , and the inverse process $+-\rightarrow-+$ with a different rate $q$. For equal densities of positive and negative particles the system exhibits three phases. For $q<1$ in the thermodynamic limit, the system organizes itself into configurations consisting of blocks of the type $000 \cdots+++\cdots---\cdots$ The dynamics out of theses states is extremely slow. Translational invariance is broken and the current vanishes. This phase is called 'pure phase'. For $1<q<q_{c}$ the system is in the 'mixed phase' which consists of two coexisting phase, the dense phase and the fluid phase. The dense phase where no vacancies exist covers a macroscopic region which shrinks to 0 for $q \rightarrow q_{c}$. It is remarkable that the current for $1<q<q_{c}$ takes the value $J=(q-1) / 4$ independent of the total density and the rate $\lambda$. For $q>q_{c}$ the fluid phase extends through the whole system. There is no charge separation and density profiles are uniform.

Similar results have been found by Evans et al. [289] in a slightly different 
model. The dynamics of their 3 -species model 20 is given by

$$
A B \underset{1}{\stackrel{q}{\rightleftarrows}} B A, \quad B C \underset{1}{\stackrel{q}{\rightleftarrows}} C B, \quad C A \underset{1}{\stackrel{q}{\rightleftarrows}} A C
$$

i.e. the rates are cyclic in $A, B$ and $C$ and the numbers $N_{A}, N_{B}$ and $N_{C}$ of particles of each species is conserved.

In the case $N_{A}=N_{B}=N_{C}$ the dynamics satisfies the detailed balance condition with respect to a Hamiltonian with long-range asymmetric interactions. Stationary states are of the form $A \cdots A B \cdots B C \cdots C$ and exhibit phase separation, i.e. for large separations $r$ the two-point function satisfies $\lim _{L \rightarrow \infty}\left[\left\langle A_{1} A_{r}\right\rangle-\left\langle A_{1}\right\rangle\left\langle A_{r}\right\rangle\right]>0$.

\subsection{Surface growth, KPZ equation and Bethe Ansatz}

In Sec. 8.1.2 we have explained how the NaSch model with $v_{\max }=1$ can be mapped onto a stochastic surface growth model. This connection can be employed to calculate several properties of the noisy Burgers and KPZ equation exactly.

Gwa and Spohn [290] used the Bethe Ansatz (see e.g. [291]) to determine the spectrum of the stochastic Hamiltionian (see App. F)

$$
\mathcal{H}=-\frac{1}{4} \sum_{j=1}^{L}\left[\boldsymbol{\sigma}_{j} \cdot \boldsymbol{\sigma}_{j+1}-1+i \epsilon\left(\sigma_{j}^{x} \sigma_{j+1}^{y}-\sigma_{j}^{y} \sigma_{j+1}^{x}\right)\right]
$$

corresponding to the ASEP with random-sequential updating and periodic boundary conditions. $\boldsymbol{\sigma}_{j}=\left(\sigma_{j}^{x}, \sigma_{j}^{y}, \sigma_{j}^{z}\right)$ are the standard Pauli matrices at site $j$ and $\epsilon$ is the asymmetry of the hopping rates, $q_{\text {right }}=\frac{1}{2}(1+\epsilon)$ and $q_{\text {left }}=\frac{1}{2}(1-\epsilon)$.

The "ground state" of $\mathcal{H}$ has eigenvalue 0 and is $L$-fold degenerate. For a fixed number $N$ of up-spins ${ }^{26}$ every configuration has equal weight in the ground state. In order to determine the dynamical scaling exponent of the noisy Burgers and KPZ equations, Gwa and Spohn investigated the finite-size behaviour of the energy gap of $\mathcal{H}$. Since $\mathcal{H}$ is non-hermitian its spectrum is complex. The first excited state is then defined as the eigenvalue with the smallest (positive) real part $E_{\text {gap }}$. In [290] it was shown that $E_{\text {gap }} \propto L^{-3 / 2}$ for $\epsilon=1$ and $N=L / 2$. This implies that the dynamical exponent $z$ for the stationary correlations of the KPZ equation is given by $z=3 / 2$. The

\footnotetext{
${ }^{25}$ One species may be interpreted as a vacant site.

${ }^{26}$ i.e. a fixed number $N$ of particles
} 
dynamical exponent relates temporal and spatial scaling behaviour on large scale. Generalizations and related results can be found in [292].

By an extension of the Bethe Ansatz method of Gwa and Spohn, Derrida and Lebowitz [293] calculated the large deviation function (LDF) of the timeaveraged current of the TASEP. The LDF is related to the total displacement $Y(t)$, i.e. the total number of hops to the right minus the total number of hops to the left between time 0 and time $t$. In the corresponding growth model $Y(t)$ is the total number of particles deposited until time $t$. The LDF is then defined as

$$
f(y)=\lim _{t \rightarrow \infty} \frac{1}{t} \ln \left[\operatorname{Prob}\left(\frac{Y(t)}{t}=\bar{v}+y\right)\right]
$$

where $\bar{v}=\lim _{t \rightarrow \infty}\left\langle Y(t) / t=\epsilon c(1-c) L^{2} /(L-1)\right.$ is the mean current for a ring of finite size $L$ and density $c=N / L$. The results of [293] have been extended and generalized in [294].

Apart from the treatment of finite systems, the BA can also be used to solve the master equation for an infinite system with a finite number of particles [295]. This allows e.g. to study the collective diffusion of two single particles.

\subsection{Protein synthesis}

You must have noticed in the earlier sections that some of the models of traffic are non-trivial generalizations or extensions of the TASEP, the simplest of the driven-dissipative systems which are of current interest in nonequilibrium statistical mechanics $[14,15,17]$. Some similarities between these systems and a dynamical model of protein synthesis have been pointed out $[296,15]$.

In a simplified picture of the mechanism of biopolymerisation ribosomes read the genetic information encoded in triplets of base pairs. They attach to one end of a messenger-RNA and then move along the chain after adding a monomer to a biopolymer attached to the ribosome. The type of monomer added depends on the genetic information read by the ribosome. When the ribosome reaches the other end of the m-RNA the biopolymer is fully synthesized and the ribosome is released.

MacDonald et al. [297] have described the kinetics of this process using an ASEP-type model. The m-RNA is represented by a chain of $L$ sites where each site corresponds to one triplet of base pairs. The ribosome is given by a hard-core particle covering $r$ neighbouring sites $(r \approx 20-30)$ which moves forward by one site with rate $q$. At the beginning of the chain particles are added with rate $\alpha q$ and at the end they are released with rate $\beta q$. In the 
idealized case $r=1$ this is exactly the TASEP of Sections 8.1.1 and 8.5. The relevant case for the experiments is $\alpha=\beta<1 / 2$. The exact solution of the TASEP allowed for an explanation of many aspects of the experiments [296].

\subsection{Granular flow}

Another quasi-one-dimensional driven-dissipative system, which is also receiving wide attention of physicists in recent years, is the granular material flowing through a pipe $[3,4]$. Since the fascinating phenomena (e.g. size segregation, convection, standing waves, localized excitations) found in granular materials have been subject of several excellent reviews [298] we discuss only briefly the similarities between the clustering of vehicles on a highway and particleparticle (and particle-cluster) aggregation process [299,300,209].

Obviously both highway traffic and granular flow through a pipe may be described as quasi-onedimensional systems consisting of discrete elements (vehicles, grains). The dynamics of these elements is determined by an intricate interplay between a driving force (driver, gravitation) and dissipation (braking, inelastic scattering processes). These similarities already show that both systems can be described by similar approaches. One important difference between traffic flow and granular flow exist, however. In granular flow density waves can move both in and against the direction of the flow whereas in traffic flow they only move backwards.

An important success of a description of granular flow using the optimal velocity model (Sec. 6.2) is the explanation of the experimentally observed $f^{-4 / 3}$ behaviour of the power spectrum [301].

\subsection{The bus route model}

The bus route model (BRM) [302] has been formulated as a one-dimensional lattice with periodic boundary conditions. The sites represent cells, each of which may be thought of as a bus stop and are labeled by an index $i$ ( $i=$ $1,2, \ldots, L)[302]$. Two binary variables $\sigma_{i}$ and $\tau_{i}$ are assigned to each cell $i$ : (i) If the cell $i$ is occupied by a bus then $\sigma_{i}=1$; otherwise $\sigma_{i}=0$. (ii) If cell $i$ has passengers waiting for a bus then $\tau_{i}=1$; otherwise $\tau_{i}=0$. Since a cell cannot have simultaneously a bus and waiting passengers, let us impose the condition that a cell cannot have both $\sigma_{i}=1$ and $\tau_{i}=1$ simultaneously. Each bus is assumed to hop from one stop to the next.

Next, let us specify the update rules (see Fig. 57): a cell $i$ is picked up at random. Then, (i) if $\sigma_{i}=0$ and $\tau_{i}=0$ (i.e, cell $i$ contains neither a bus nor 


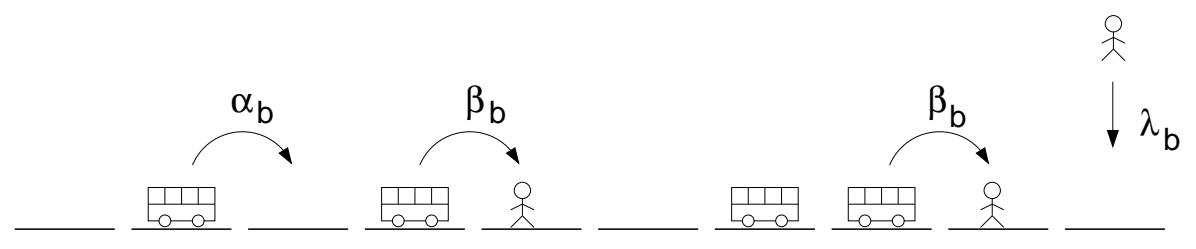

Fig. 57. The bus-route model (from [302]).

waiting passengers), then $\tau \rightarrow 1$ with probability $\lambda$, where $\lambda$ is the probability of arrival of passenger(s) at the bus stop. (ii) If $\sigma_{i}=1$ (i.e., there is a bus at the cell $i$ ) and $\sigma_{i+1}=0$, then the hopping rate $\mu$ of the bus is defined as follows: (a) if $\tau_{i+1}=0$, then $\mu=\alpha_{b}$ but (b) if $\tau_{i+1}=1$, then $\mu=\beta_{b}$, where $\alpha_{b}$ is the hopping rate of a bus onto a stop which has no waiting passengers and $\beta_{b}$ is the hopping rate onto a stop with waiting passenger(s). Generally, $\beta_{b}<\alpha_{b}$, which reflects the fact that a bus has to slow down when it has to pick up passengers. We can set $\alpha_{b}=1$ without loss of generality. When a bus hops onto a stop $i$ with waiting passengers $\tau_{i}$ is reset to zero as the bus takes all the passengers. Note that the density of buses $c=N / L$ in a conserved quantity whereas that of the passengers is not.

An ideal situation in this bus-route model would be one where the buses are evenly distributed over the route so that each bus picks up roughly the same number of passengers. However, because of some fluctuation, a bus may be delayed and, consequently, the gap between it and its predecessor will be larger than the average gap. Therefore, this bus has to pick up more passengers than what a bus would do on the average, because during the period of delay more passengers would be waiting for it and, as a result, it would get further delayed. On the other hand, the following bus has to pick up fewer passengers than what a bus would do on the average and, therefore, it would catch up with the delayed bus from behind. The slowly moving delayed bus would slow down the buses behind it thereby, eventually, creating a jam. In other words, once a larger-than-average gap opens up between two successive buses, the gap is likely grow further and the steady state in a finite system would consist of a single jam of buses and one large gap. This is very similar to the Bose-Einsteincondensation-like phenomenon we have observed earlier in particle-hopping models with slow impurities. On the basis of heuristic arguments and meanfield approximation it has been argued [302] that this model exhibits a true phase transition from an inhomogeneous low-density phase to a homogeneous (but congested) high-density phase only in the limit $\lambda \rightarrow 0$. Finally we mention that the BRM with parallel dynamics has been recently been studied in [303] where also its connection with the NaSch model has been elucidated. 


\subsection{Mobile directional impurities}

We have considered the effects of random hopping probabilities, assigned either to the lattice sites $[214,137,190]$ or to the particles $[204-206]$ in the TASEP and in the NaSch-type models of vehicular traffic, on the nature of the corresponding steady states as well as their approach to the steady-states starting from random initial conditions. Toroczkai and Zia [304] solved analytically a model with one "mobile directional impurity"; this model is also an extension of the ASEP. In this model, $N$ particles, labeled by integers 1 to $N$ (from left to right), occupy the sites of a one-dimensional lattice of length $N+1$ where periodic boundary conditions are applied. Thus, there is a single "hole" (i.e., empty site) in this model. The shifting of the hole from the site in between the particles $n$ and $n+1$ to the site in between particles $n+1$ and $n+2$ is described by the statement "hole jump from position $n$ to $n+1$ ". In the absence of any impurity, the hole at position $n$ can exchange position with either the particle on its left (with probability $W_{n-1, n}$ ) or the particle on its right (with probability $\left.W_{n+1, n}\right)$. These probabilities are arbitrary and direction-dependent (i.e., in general, $\left.W_{n-1, n} \neq W_{n+1, n}\right)$ but time-independent. Note that the hopping probabilities of the hole is determined by the particles (rather than the lattice sites) in front and behind it. The general case, where $W_{n-1, n} \neq W_{n+1, n}$ and the probabilities $W_{n-1, n}$ as well as $W_{n+1, n}$ for different $n$ are chosen randomly, is referred to as the random asymmetric case. As the hole wanders, the string of particles also shifts as a whole. However, this system is translationally invariant in the sense that the jump rate of any particular particle-hole pair is independent of its location on the lattice.

The "directional impurity" is introduced by identifying a specific bond (whose position is fixed with respect to the lattice) as a defect bond such that the time-independent rates of particle-hole exchanges across it are fixed at, say, $q$ and $q^{\prime}$ irrespective of the particle-hole pair involved. In other words, when the hole is in between the particles $n$ and $n+1$, the hopping probability to the right (left) is always $W_{n+1, n}\left(W_{n-1, n}\right)$, except when the defect bond is involved. This model can be represented as in Fig. 58 where the defect bond is shown as a kink; the motivation for such a kink came from an earlier model [305] of gel electrophoresis [306].

More specifically, suppose, the probabilities for the exchange of the hole with a particle across the defect bond are $q$ if the hole moved upward and $q^{\prime}$ if the hole moved downward, independent of the particle involved. In the socalled pure limit, we have, for all $n, W_{n+1, n}=W_{\rightarrow}$ and $W_{n-1, n}=W_{\leftarrow}$. In this limit, the hole can also be regarded as a particle undergoing biased diffusion (if $\left.W_{\rightarrow} \neq W_{\leftarrow}\right)$ everywhere except across across a specific defect bond. If we make the further assumption that no backward motion of any particle is allowed, then this model reduces to the model of TASEP with a single blockage [214]. 
(a)

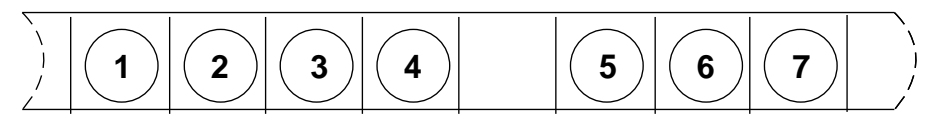

(b)

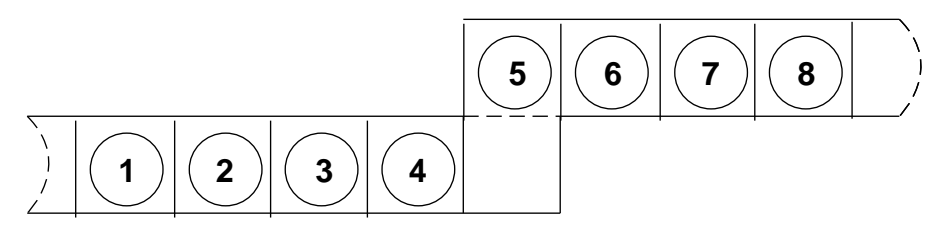

Fig. 58. (a) The translationally-invariant Toroczkai-Zia model without any defect bond. (b) The translational invariance is broken by the defect bond (represented by the kink denoted by the dashed line).

\subsection{Computer networks}

Inspired by the recent success of the methods of statistical mechanics outside the traditional domain of physics, tools of statistical mechanics have also been applied to analyze fundamental properties of information traffic on the international network of computers (Internet) [307-310]. Messages in the form of information packets are continuously being emitted from the hundreds of millions of host computers and transported to their destination computers through this network. Each of these packets is relayed through the so-called routers on its way. The routers can deal with the packets one by one. Each router has a finite buffer where the arriving packets get queued up and forwarded one by one from the head of the remaining queue to their respective next destinations. Since packets run with the velocity of light through the cables, information congestion does not take place inside the cables. It is the routers which give rise to the information congestion on the internet. Measuring the fluctuations in the round-trip time taken by a message on the internet (using the ping command of the UNIX operating system), $1 / f$-like power spectrum has been observed [307,308].

In the square lattice model of a computer network developed by Ohira and Sawatari [310] information packets are generated at the sites on the boundary at a rate $\lambda$ with the corresponding destination addresses chosen randomly from among the boundary sites. The packets can form queues of unlimited length at the the inner nodes, which act as routers of the network. At every time step, the packets from the heads of the queues at the routers are forwarded to the tail ends of the queues at the next router. Both deterministic and probabilistic strategies have been considered for selecting the next router to which the individual packets are to be forwarded. On reaching their individual destinations the packets die. The average number of time steps between the birth and death of a packet is referred to as the average lifetime of a packet. Computing the average lifetime as a function of the birth rate $\lambda$ of the packets, Ohira and Sawatari [310] observed a transition from a low-congestion phase 
to a high-congestion phase at a non-zero finite value $\lambda_{*}$.

\section{Summary and conclusion}

In this section we summarize our conclusions regarding the current status of understanding of the statistical physics of vehicular traffic. We also speculate on the future trends of research in this area.

As we stated in the beginnning, one of the main aims of basic research on vehicular traffic, from the point of view of statistical physics, is to understand the nature of the steady-states of the system. We have summarized the empirical evidences available at present in support of the occurrence of three distinct dynamical phases, namely, those corresponding to free-flow, synchronized flow and stop-and-go traffic. Our critical review of the theoretical works has made it clear that, at present, the physical mechanisms at the "microscopic" level, which give rise to the synchronized traffic, are not as well understood as those responsible for the free-flow and stop-and-go traffic. There are, however, strong indications that for a complete theory, which would account for all these three phases, one must take into account not only the vehicles on a given stretch of the highway but also on the on- and off-ramps.

We have explored the possibility of transitions from one dynamical phase to another in the NaSch model (and TASEP) with periodic boundary conditions. Moreover, we have also presented the generic phase diagram of the TASEP with open boundary conditions and explained the notion of boundary-induced phase transitions in such one-dimensional driven-diffusive lattice gases which are far from equilibrium. Furthermore, we have found that while some models exhibit first-order phase transitions, some others exhibit second order phase transitions and the signatures of "criticality" while in some rare situations, e.g., in the cruise-control limit of the NaSch model, the system is found to exhibit even "self-organized" criticality.

In the beginning, we stated that one of the aims of basic research on vehicular traffic is to understand the nature of the dynamical fluctuations around the steady-states. The time-dependent correlations functions and the distributions of the relaxation times have been computed for the NaSch model and some other models but the general questions of the validity of dynamic scaling and dynamic universality classes have not been addressed. Another aim of the statistical mechanical approach to vehicular traffic, as we stated also in the beginning, is to investigate how the system evolves from initial states which are far from the corresponding steady-state. The phenomenon of "coarsening" of the platoons of vehicles during evolution from random initial states have been studied in some models. But, the questions of "universality", if any, of 
the growth exponents have not been addressed so far. Metastable states have been observed in several CA models. But, to our knowledge, the mechanisms of spontaneous decay of such states (analogue of homogeneous nucleation) has not been investigated so far. Besides, to our knowledge, so far it has not been possible to develope any powerfull analytical technique for calculating the dynamical properties of the traffic models.

While stating the aims of basic research on vehicular traffic, we also mentioned the need to understand the effects of quenched disorder on the steady-states as well as on the dynamical properties of the systems. We have seen that the randomization of the hopping probability of the vehicles can lead to some exotic platooning phenomena which are close analogs of the "Bose-Einsteincondensation". An alternative prescription for introducing quenched disorder into the traffic models is to install random bottlenecks on the road and assign a time-independent hopping probability (or, equivalently, a "permeability") for hopping across bonds in such locations. It has been found that such localized bottlenecks can lead to global phase-segregation.

The NaSch model is the most extensively studied minimal CA model of vehicular traffic on idealized single-lane highways. We have explained the conceptual framework, and illustrated the use of the mathematical formalism, of the cluster-theoretic analytical calculations for the NaSch model. This formalism, which yields exact results for the NaSch model with $v_{\max }=1$, gives quite accurrate estimates of various quantities of interest as long as $v_{\max }$ is not too large. It would be desirable to develope a new formalism to carry out exact analytical calculations for higher velocities too. Here also the limit $v_{\max }=\infty$ is interesting since it shows a rather peculiar behaviour.

In addition to the detailed discussions on the NaSch model and its various generalizations, we have also mentioned briefly some other alternative CA models of single-lane highway traffic, e.g., the VDR model, the Fukui-Ishibashi model, the Werth-Froese-Wolf model, etc. so that one can appreciate the ongoing efforts to formulate the most satisfactory minimal model.

From the point of view of practical applications, modelling vehicular traffic on multi-lane highways are more relevant than that on idealized single-lane highways which are, nevertheless, interesting from the point of fundamental understanding of truly non-equilibrium phenomena in driven-diffusive lattice gases. At present, there are several different alternative prescriptions for formulating the CA rules for lane-changing of the vehicles on multi-lane highways. But, in order to pick out the most appropriate one from among these CA models theorists would require input from careful further observations of the phenomenon of lane-changing on real multi-lane highways. Empirical observations may also indicate modifications or extensions of the CA rules necessary for more realistic modelling of the multi-lane traffic. 
The generalizations of the CA models of traffic on idealized single-lane highways to those on multi-lane highways may be regarded as extensions of onedimensional model chains to one-dimensional strips. The BML model of vehicular traffic in cities may be regarded as a further generalizations of these models from one-dimensional chains to two-dimensional lattices, or further, to decorated lattices. A few different CA rules have been considered so far for taking into account the effects of the traffic lights at the crossings of streets in such idealized street networks. We have emphasized the intrinsic differences between percolation clusters and the cluster of jammed vehicles in the BML (and similar) models in spite of some apparent similarities between them.

We have focused attention mainly on the progress made in the recent years using "particle-hopping" models, formulated in terms of cellular automata, and compared these with several other similar systems. Although this may be a slightly biased overview (as all reviews usually are) of the theory of vehicular traffic, we have also discussed the main ideas behind all the major approaches including the fluid-dynamical, gas-kinetic and car-following theories of vehicular traffic. At present the relationships between different approaches of modelling have not explored in great detail. It would be very useful if the phenomenological parameters of the macroscopic theories can be estimated by utilizing the mathematical formulae relating these with those of the "microscopic" models.

It is now quite clear that, in order to make significant further progress, we not only need more realistic models and better techniques of calculation but we also need more detailed and accurate empirical data from real traffic on highways as well as more careful re-analysis of the existing data in the light of recently developed concepts. So far as the observation of the real traffic is concerned, a lot can be learnt from a systematic analysis of aereal pictures or video photographs. Alternatively, as the second best choice, a series of counting loops along the highway can give more insight, by providing detailed information on, for example, time-headways (flux), velocity and local density.

If you are a critical thinker (or a pragmatist) you may ask: "armed with the theoretical tools at our disposal now, can we predict the occurrence of a traffic jam at a specific place on a given highway (or street) at a particular instant of time"? This question sounds similar to questions often asked in the context of some other interdisciplinary topics of current research in the area of complex systems, e.g., "can we predict an earthquake", or, "can we predict a stock market crash"? Of course, we know that, at present, the best we can hope for is to predict (if at all possible) probabilities of occurrences of all these phenomena. But, we must admit that, we have a long way to go before we come even close to this goal. Nevertheless, we hope you have enjoyed the fascinating twists and turns of the way we have covered so far. Our endeveour will be more successful if your interest has been stimulated by the intellectual 
challenges posed by the open problems and if you are willing to uncover the current mysteries as well as anticipating new surprises that may lie ahead. We are just at the beginning of a long road!

"The volume of vehicular traffic in the past several years has rapidly outstripped the capacities of the nation's highways. It has become increasingly necessary to understand the dynamics of traffic flow and obtain a mathematical description of the process" - H. Greenberg (1959)

Acknowledgements: It is our pleasure to thank P. Arndt, R. Barlovic, M. Barma, J.G. Brankov, E. Brockfeld, G. Diedrich, B. Eisenblätter, J. Esser, K. Ghosh, N. Ito, J. Kertész, K. Klauck, W. Knospe, S. Krauss, J. Krug, D. Ktitarev, A. Majumdar, K. Nagel, L. Neubert, A. Pasupathy, V. Popkov, V.B. Priezzhev, N. Rajewsky, M. Schreckenberg, G. Schütz, S. Sinha, D. Stauffer, R.B. Stinchcombe, Y. Sugiyama, P. Wagner, D.E. Wolf and J. Zittartz for enjoyable collaborations the results of some of which have been reviewed here, for useful discussions and for critical comments as well as suggestions on a preliminary draft of this review. One of us (DC) acknowledges warm hospitality of ICTP, Trieste during the preparation of this manuscript. This work is supported by SFB341 Köln-Aachen-Jülich. 


\section{A Definition of update orders}

A dynamical model is not fully defined just by its local transition rules. In addition one has to specify the order in which the rules are applied to the different particles, i.e. the update ordering (sometimes also called 'dynamics'). This is an essential part of the definition of the model since the transient and even the stationary state may differ dramatically [311,312].

For the NaSch model one uses a parallel update scheme [140] where the rules are applied to all particles (i.e., vehicles) at the same time. This kind of ordering is sometimes also called synchronous updating.

Among the various types of asynchronous update schemes most frequently the the so-called random-sequential update is used. Here one picks one particle at random and applies the transition rules to it. Then one makes another random choice (which can also be the same particle again) and so on. This update is sometimes called continuous since it can be described by a master equation in continuous time.

Apart from the parallel update there are other updates which are discrete in time. We just mention the ordered-sequential updates. Here one starts by applying the transition rules to one particle. After that the rules are applied to the other particles in a fixed order, e.g. one might continue with the next particle ahead of the first one (forward-ordered) or the next particle behind it (backward-ordered). We would like to point out that, in principle, one has to distinguish two different types of ordered-sequential updates which one could name site-ordered-sequential and particle-ordered-sequential, respectively. In contrast to the particle-ordered-sequential update described above, in the siteordered-sequential update the rules are applied to all sites consecutively. This might have a strong effect, since a particle might move to next cell ahead which then is updated next (for the forward-particle-ordered-sequential update). Then this particle might move again and so on. This is different from the particle-ordered-sequential case where a particle at most moves once during a sweep through the lattice. As an example consider the extreme case of the NaSch mode with $v_{\max }=1$ where only one particle is present which moves with probability $q=1$ to an empty cell in front. This particle will move through the whole lattice during one sweep! By looking at a lattice with two particles, one can already see that the two different updates might introduce rather different correlations. Starting with particles separated by $d$ empty sites, in the site-ordered-sequential update the left particle will move to the right until it reaches the right particle, which then starts to move. On the other hand, in the case of particle-ordered-sequential update the particles will stay always $d$ or $d-1$ sites apart. For general values of $q$ the situation is similar. 
There are several other updates which can be defined. We refer to the literature (see e.g. [166,311-315]) for a comparison of different update procedures. The parallel update usually produces the strongest correlations and is used for traffic simulations [140]. Note that the forward-particle-ordered-sequential update is almost identical to the parallel update. In the case of periodic boundary conditions a difference only occurs during the update of the last particle. In the forward-ordered case the particle in front of it (i.e. the first particle) might already have moved since it has been updated earlier. Although this difference appears to be minor it can have a large effect. The difference between parallel and forward-particle-ordered update can be viewed as a dynamical defect.

\section{B TASEP}

This simple model of driven systems of interacting particles is one of the most exhaustively studied prototype models in nonequilibrium statistical mechanics [14-16,316,317]. This model can be divided into four classes on the basis of the boundary conditions and the update scheme for the implementation of the dynamics. In this appendix we consider the TASEP with only randomsequential dynamics.

Let us consider the TASEP with periodic boundary conditions and randomsequential dynamics. Since only two states, namely empty and occupied, are allowed for each site we can use a two-state variable $n_{i}$ to denote the state of the $i$-th site where $n_{i}=0$ if the $i$-th site is empty and $n_{i}=1$ if the $i$ th site is occupied. For any given initial configuration $\left\{n_{i}(0)\right\}$, we can write the equations governing the time evolution of $\left\langle n_{i}(t)\right\rangle$ (and all the correlation functions) by taking into account all the processes during the elementary time interval $d t$. It is not difficult to establish that

$$
\begin{gathered}
n_{i}(t+d t)= \begin{cases}n_{i}(t) & \text { with probability } 1-2 d t \\
n_{i-1}(t)+n_{i}(t)-n_{i-1}(t) n_{i}(t) & \text { with probability } d t \\
n_{i}(t) n_{i+1}(t) & \text { with probability } d t\end{cases} \\
\qquad \frac{d n_{i}}{d t}=n_{i-1}\left(1-n_{i}\right)-n_{i}\left(1-n_{i+1}\right)
\end{gathered}
$$

which, upon averaging over the history between times 0 and $t$, leads to the equation

$$
\frac{d\left\langle n_{i}\right\rangle}{d t}=\left\langle n_{i-1}\right\rangle-\left\langle n_{i}\right\rangle-\left\langle n_{i-1} n_{i}\right\rangle+\left\langle n_{i} n_{i+1}\right\rangle
$$


for $\left\langle n_{i}\right\rangle$, the average occupation of the $i$-th site. Note that the equation for $\left\langle n_{i}\right\rangle$ involves two-site correlations. Similarly, it is straightforward to see that the equations for the two-site correlations involve three-site correlations and so on. Thus, the problem is an intrinsically $N$-body problem! The probability distribution for this system in the steady-state is given by [22]

$$
P_{\text {steady-state }}\left(\left\{n_{i}\right\}\right)=\frac{N !(L-N) !}{L !}
$$

where $L$ and $N$ refer to the total number of sites and the total number of particles, respectively. From this distribution it follows that $\left\langle n_{i}\right\rangle=N / L$ and

$\langle v\rangle=\frac{L-N}{L-1}$ which lead to $\left\langle n_{i}\right\rangle=c$ and $\langle v\rangle=(1-c)$ in the thermodynamic limit.

The stationary state of the TASEP with open boundary conditions and randomsequential dynamics has been determined exactly using the so-called Matrix Product Ansatz (MPA) (see Appendix F for a more technical introduction) in [167] and in [168] using recursion relations. This solution has been generalized to different types of discrete dynamics in $[172,173,166]$. The solution for parallel dynamics was obtained recently in [164] and [165] using generalizations of the MPA technique.

\section{Naive site-oriented mean-field treatment of the NaSch model}

Suppose, $c_{v}(i, t) \equiv$ Probability that there is a vehicle with speed $v(v=$ $\left.0,1,2, \ldots, v_{\max }\right)$ at the site $i$ at the time step $t$. Then, obviously, $c(i, t)=$ $\sum_{j=0}^{v_{\max }} c_{j}(i, t) \equiv$ Probability that the site $i$ is occupied by a vehicle at the time step $t$ and $d(i, t)=1-c(i, t)$ is the corresponding probability that the site $i$ is empty at the time step $t$. Using the definition

$$
J(c, p)=\sum_{v=1}^{v_{\max }} v c_{v}
$$

for the flux $J(c, p)$ one can get the mean-field fundamental diagram for the given $p$ provided one can get $c_{v}$ in the mean-field approximation.

Step I: Acceleration stage $\left(t \rightarrow t_{1}\right)$

$$
\begin{aligned}
c_{0}\left(i, t_{1}\right) & =0, \\
c_{v}\left(i, t_{1}\right) & =c_{v-1}(i, t), \quad\left(0<v<v_{\max }\right) \\
c_{v_{\max }}\left(i, t_{1}\right) & =c_{v_{\max }}(i, t)+c_{v_{\max }-1}(i, t)
\end{aligned}
$$

Step II: Deceleration stage $\left(t_{1} \rightarrow t_{2}\right)$ 


$$
\begin{aligned}
c_{0}\left(i, t_{2}\right)= & c_{0}\left(i, t_{1}\right)+c\left(i+1, t_{1}\right) \sum_{v=1}^{v_{\max }} c_{v}\left(i, t_{1}\right) \\
c_{v}\left(i, t_{2}\right)= & c\left(i+v+1, t_{1}\right) \prod_{j=1}^{v} d\left(i+j, t_{1}\right) \sum_{v^{\prime}=v+1}^{v_{\max }} c_{v^{\prime}}\left(i, t_{1}\right) \\
& +c_{v}\left(i, t_{1}\right) \prod_{j=1}^{v} d\left(i+j, t_{1}\right), \quad\left(0<v<v_{\max }\right) \\
c_{v_{\max }}\left(i, t_{2}\right)= & \prod_{j=1}^{v_{\max }} d\left(i+j, t_{1}\right) c_{v_{\max }}\left(i, t_{1}\right)
\end{aligned}
$$

Step III: Randomization stage $\left(t_{2} \rightarrow t_{3}\right)$

$$
\begin{array}{rlrl}
c_{0}\left(i, t_{3}\right) & =c_{0}\left(i, t_{2}\right)+p c_{1}\left(i, t_{2}\right) & \\
c_{v}\left(i, t_{3}\right) & =q c_{v}\left(i, t_{2}\right)+p c_{v+1}\left(i, t_{2}\right), & & \left(0<v<v_{\max }\right) \\
c_{v_{\max }}\left(i, t_{3}\right) & =q c_{v_{\max }}\left(i, t_{2}\right) &
\end{array}
$$

Step IV: Movement stage $\left(t_{3} \rightarrow t+1\right)$

$$
c_{v}(i, t+1)=c_{v}\left(i-v, t_{3}\right), \quad\left(0 \leq v \leq v_{\max }\right)
$$

In the special case $v_{\max }=1$ the equations (C.1-C.10) get simplified and, hence, we get

$$
\begin{aligned}
& c_{0}(i, t+1)=c(i, t) c(i+1, t)+p c(i, t) d(i+1, t), \\
& c_{1}(i, t+1)=q c(i-1, t) d(i, t) .
\end{aligned}
$$

Similarly, in the case of $v_{\max }=2$, one gets

$$
\begin{array}{rr}
c_{0}(i, t+1)=[c(i, t)+p d(i, t)] c_{0}(i, t)+[1+p d(i, t)] c(i, t)\left[c_{1}(i, t)+c_{2}(i, t)\right], & (\mathrm{C} .13) \\
c_{1}(i, t+1)=d(i, t) q c_{0}(i, t)+d(i, t)[q c(i, t)+p d(i, t)]\left[c_{1}(i, t)+c_{2}(i, t)\right], \\
c_{2}(i, t+1)=q d^{2}(i, t)\left[c_{1}(i, t)+c_{2}(i, t)\right] .
\end{array}
$$

\section{Paradisical mean-field theory}

For $v_{\max }=1$ the question, whether a state is a GoE state or not, can be decided locally by investigating just nearest-neighbour configurations. By analysing 
the update rules one finds that all states containing the local configurations $(0,1)$ or $(1,1)$, i.e. configurations where a moving vehicle is directly followed by another car, are GoE states. This is not possible as can be seen by looking at the previous configurations. The momentary velocity gives the number of cells that the car moved in the previous timestep. In both configurations the first car moved one cell. Therefore it is immediately clear that $(0,1)$ is a GoE state since otherwise there would have been a doubly-occupied cell before the last timestep. The configuration $(1,1)$ is also not possible since both cars must have occupied neighbouring cells before the last timestep too. Therefore, according to step 2, the second car could not move.

The MFT equations (64) and (65) have to be modified to take into account the existence of GoE states. In general, one has to follow the procedure outlined in Appendix C. A quicker way to derive the paradisical mean-field (pMF) equations is to analyse the MF equations (64) and (65). In (64) the contribution $c(i ; t) c(i+1 ; t)$ appears. Since we know that site $i+1$ can never be occupied by a car with velocity 1 if site $i$ is not empty, this contribution has to be modified to $c(i ; t) c_{0}(i+1 ; t)$ in pMFT. All other contributions are left unchanged compared to MFT.

Due to this modification and the corresponding reduction of the configuration space the normalization $c_{0}+c_{1}=c$ is no longer satisfied automatically. Therefore a normalization constant $\mathcal{N}$ has to be introduced. The final equations for a homogeneous stationary state are than given by

$$
\begin{aligned}
& c_{0}=\mathcal{N}\left(c_{0}+p d\right) c \\
& c_{1}=\mathcal{N} q c d
\end{aligned}
$$

with the normalization

$$
\mathcal{N}=\frac{1}{c_{0}+d}
$$

Since $c_{0}+c_{1}=c$ we have only one independent variable for fixed density $c$, e.g. $c_{1}$. Solving (D.1), (D.2) for $c_{1}$ we obtain

$$
c_{1}=\frac{1}{2}(1-\sqrt{1-4 q(1-c) c})
$$

The flow is given by $f(c)=c_{1}$ and we recover the exact solution for the case $v_{\max }=1$.

In the case $v_{\max }=2$ more GoE states exist. In order to identify these it is helpful to note that the rules step 1 - step 4 imply $d_{j}(t)=d_{j}(t-1)+v_{j+1}(t)-$ $v_{j}(t)$ and therefore 


$$
\begin{aligned}
& d_{j}(t) \geq v_{j+1}(t)-v_{j}(t) \\
& v_{j}(t) \leq d_{j}(t-1)
\end{aligned}
$$

The second inequality (D.6) is a consequence of step 2.

In the following we list the elementary GoE states, i.e. the local configurations which are dynamically forbidden (cars move from left to right):

$$
\begin{array}{ccc}
(0,1), \quad(0,2), & (1,2), & (0, \bullet, 2), \\
(1,1), \quad(2,1), \quad(2,2), \quad(1, \bullet, 2), & (2, \bullet, 2), \\
& (0, \bullet, \bullet, 2) .
\end{array}
$$

The numbers give the velocity of a vehicle in an occupied cell and $\bullet$ denotes an empty cell.

The elementary GoE states in (D.7) violate the inequality (D.5), and the configurations in (D.8) violate (D.6). The state in (D.9) is a second order GoE state. Going one step back in time leads to a first order GoE state since $(0, \bullet, \bullet, 2)$ must have evolved from $(0, v)$ (with $v=1$ or $v=2$ ).

Again we can derive the pMF equations by modifying the method for the derivation of the MFT. Taking into account only the first order GoE states (D.7) and (D.8) one obtains the following pMF equations:

$$
\begin{aligned}
& c_{0}=\mathcal{N}\left[c_{0} c+p d\left(c_{0}+c_{1} c\right)\right], \\
& c_{1}=\mathcal{N}\left[p d^{2}\left(c_{1}+c_{2}\right)+q d\left(c_{0}+c_{1} c\right)\right], \\
& c_{2}=\mathcal{N} q d^{2}\left(c_{1}+c_{2}\right) .
\end{aligned}
$$

The normalization $\mathcal{N}$ ensures $c_{0}+c_{1}+c_{2}=c$ and is given explicitly by

$$
\mathcal{N}=\frac{1}{c_{0}+d c_{1}+d^{2} c_{2}}=\frac{1}{c_{0}+d\left(1-c_{2}\right)}
$$

These equations have been analysed in [142]. After expressing $c_{2}$ through $c_{0}$ and $c$ by

$$
c_{2}=\frac{1}{2 d}\left(c_{0}+d-\sqrt{\left(c_{0}+d\right)^{2}-4 q d^{3}\left(c-c_{0}\right)}\right) .
$$

Inserting this result into (D.10) we obtain a cubic equation which determines $c_{0}$ in terms of the parameters $c$ and $p$ [142]. Results for different values of $p$ are shown in Fig. 19. These results are only slightly modified when also the second order GoE state is taken into account [142]. 


\section{E Equations of car-oriented theory of NaSch Model and COMF approximation}

In terms of $p, q, g$ and $\bar{g}(t)=1-g(t)$, the equations describing the time evolution of the probabilities $P_{n}(t)$ for the NaSch model with $v_{\max }=1$ are given by

$$
\begin{aligned}
& P_{0}(t+1)=\bar{g}(t)\left[P_{0}(t)+q P_{1}(t)\right], \\
& P_{1}(t+1)=g(t) P_{0}(t)+[q g(t)+p \bar{g}(t)] P_{1}(t)+q \bar{g}(t) P_{2}(t), \\
& P_{n}(t+1)=p g(t) P_{n-1}(t)+[q g(t)+p \bar{g}(t)] P_{n}(t)+q \bar{g}(t) P_{n+1}(t) \quad(n \geq 2) .
\end{aligned}
$$

It is worth mentioning here that, for the NaSch model with $v_{\max }=1$, the 2-cluster probabilities $P_{2}\left(\sigma_{i}, \sigma_{j}\right)$ of the the SOMF theory are related to the probabilities $P_{n}$ of the COMF theory through

$$
\begin{aligned}
& P_{2}(1,1)=c P_{0}, \\
& P_{2}(1,0)=c(1-c) P_{1}, \\
& P_{2}(0,0)=(1-c) \frac{P_{n+1}}{P_{n}} \quad(n \geq 1)
\end{aligned}
$$

\section{F The matrix-product Ansatz for stochastic systems}

For the stochastic systems considered here the time-evolution of the probability $P(\boldsymbol{\tau}, t)$ to find the system in the configuration $\boldsymbol{\tau}=\left(\tau_{1}, \ldots, \tau_{L}\right)$ is determined by the master equation. For random-sequential dynamics it has the form

$$
\frac{\partial P(\boldsymbol{\tau}, t)}{\partial t}=\sum_{\tilde{\boldsymbol{\tau}}} w(\tilde{\boldsymbol{\tau}} \rightarrow \boldsymbol{\tau}) P(\tilde{\boldsymbol{\tau}}, t)-\sum_{\tilde{\boldsymbol{\tau}}} w(\boldsymbol{\tau} \rightarrow \tilde{\boldsymbol{\tau}}) P(\boldsymbol{\tau}, t)
$$

with transition rates $w(\tilde{\boldsymbol{\tau}} \rightarrow \boldsymbol{\tau})$ from state $\tilde{\boldsymbol{\tau}}$ to state $\boldsymbol{\tau}$. Eq. (F.1) can be rewritten in the form of a Schrödinger equation in imaginary time [318],

$$
\frac{\partial}{\partial t}|P(t)\rangle=-\mathcal{H}|P(t)\rangle,
$$

with the state vector $|P(t)\rangle=\sum_{\boldsymbol{\tau}} P(\boldsymbol{\tau}, t)|\boldsymbol{\tau}\rangle$. The vectors $|\boldsymbol{\tau}\rangle=\left|\tau_{1}, \ldots, \tau_{L}\right\rangle$ corresponding to the configurations $\tau$ form an orthonormal basis of the configuration space. The stochastic Hamiltonian $\mathcal{H}$ is defined through its matrix 
elements

$$
\langle\boldsymbol{\tau}|\mathcal{H}| \tilde{\boldsymbol{\tau}}\rangle=-w(\tilde{\boldsymbol{\tau}} \rightarrow \boldsymbol{\tau}), \quad\langle\boldsymbol{\tau}|\mathcal{H}| \boldsymbol{\tau}\rangle=\sum_{\boldsymbol{\tau} \neq \tilde{\boldsymbol{\tau}}} w(\boldsymbol{\tau} \rightarrow \tilde{\boldsymbol{\tau}}) \quad(\boldsymbol{\tau} \neq \tilde{\boldsymbol{\tau}})
$$

The stationary state of the stochastic process corresponds to the eigenvector $\left|P_{0}\right\rangle$ of $\mathcal{H}$ with eigenvalue 0 .

For discrete-time dynamics the master equation takes the form

$$
P(\boldsymbol{\tau}, t+1)=\sum_{\tilde{\boldsymbol{\tau}}} W(\tilde{\boldsymbol{\tau}} \rightarrow \boldsymbol{\tau}) P(\tilde{\boldsymbol{\tau}}, t)
$$

where $W(\tilde{\boldsymbol{\tau}} \rightarrow \boldsymbol{\tau})=w(\tilde{\boldsymbol{\tau}} \rightarrow \boldsymbol{\tau}) \cdot \Delta t$ are transition probabilities. This can be rewritten as

$$
|P(t+1)\rangle=\mathcal{T}|P(t)\rangle
$$

Here the stationary state corresponds to the eigenvector $\left|P_{0}\right\rangle$ of the transfer matrix $\mathcal{T}$ with eigenvalue 1.

A very powerful method for the determination of stationary solutions of the master equation is the so-called matrix-product Ansatz (MPA). For a system with open boundaries the weights $P(\boldsymbol{\tau})$ in the stationary state can be written in the form

$$
P\left(\tau_{1}, \ldots, \tau_{L}\right)=\frac{1}{Z_{L}}\left\langle W\left|\prod_{j=1}^{L}\left[\tau_{j} D+\left(1-\tau_{j}\right) E\right]\right| V\right\rangle .
$$

For periodic boundary condition the MPA takes the form

$$
P\left(\tau_{1}, \ldots, \tau_{L}\right)=\operatorname{Tr}\left(\prod_{j=1}^{L}\left[\tau_{j} D+\left(1-\tau_{j}\right) E\right]\right) .
$$

For simplicity we have assumed a two-state system where e.g. $\tau_{j}=0$ corresponds to an empty cell $j$ and $\tau_{j}=1$ to an occupied cell. $Z_{L}$ is a normalization constant that can be calculated as $Z_{L}=\left\langle W\left|C^{L}\right| V\right\rangle$. In (F.6), (F.7) $E$ and $D$ are matrices and $\langle W|$ and $|V\rangle$ are vectors characterizing the boundary conditions. The explicit form of these quantities has to be determined from the condition that (F.6) or (F.7) solves the master equation. This leads in general to a algebraic relations between the matrices $E$ and $D$ and the boundary vectors $\langle W|$ and $|V\rangle$. Once one these have been determined one has a simple recipe for determining $P\left(\tau_{1}, \ldots, \tau_{L}\right)$ : First, translate the configuration $\tau_{1}, \ldots, \tau_{L}$ into a product of matrices by identifying each empty cell $\left(\tau_{j}=0\right)$ with a factor $E$ and each occupied cell $\left(\tau_{j}=1\right)$ with $D$. In that way the configuration $011001 \cdots$ corresponds to the product $E D D E E D \cdots=E D^{2} E^{2} D \cdots$. The weight of the configuration is then just the matrix element with the vectors $\langle w|$ and $|v\rangle$. 
A simple example is the ASEP discussed in Sect. 8.1.1. Here these quantities have to satisfy

$$
\begin{aligned}
p D E & =D+E, \\
\alpha\langle W| E & =\langle W|, \\
\beta D|V\rangle & =|V\rangle .
\end{aligned}
$$

If one is able to find explicit representations for this algebra one can determine in principle all expectation values in the stationary state exactly. For (F.8)(F.10) one can show that all representations are infinite-dimensional [167]. Only on the line $\alpha+\beta=p$ one-dimensional representations (with $E, D$ and $\langle W|| V$,$\rangle being real numbers) are possible 27$.

At this point it might appear that the MPA works only in very special cases. However, it can be shown that the stationary state of one-dimensional stochastic processes is generically of matrix-product form [319-321] 28 . Even if it is not straightforward to find general representations of the resulting algebras, one can at least search systematically for finite-dimensional representations on special lines in the parameter space of the model. Furthermore, since the mathematical structure of the stationary state is known it is sometimes possible to derive rather general results. As an example in [320] interesting relations between expectation values for ordered-sequential and sublattice-parallel dynamics have been derived.

For a more detailed description of the MPA for different types of dynamics and its relation with the MPA technique for quantum-mechanical spin systems [322] we refer to [166]. A review of the treatment of the ASEP using the MPA is given in [22]. The MPA has also been extended to treat the full dynamics, not only the stationary state [323]. Using time-dependent matrices $D(t)$ and $E(t)$ one obtains the Bethe Ansatz equations for the corresponding stochastic Hamiltonian [323-325].

\section{G Two schemes for solving the mean-field approximation of the DTASEP}

A mean-field approximation scheme for this model has also been developed [137]. The time-averaged steady-state current $J_{j, j+1}$ in the bond $(j, j+1)$ is given by $J_{j, j+1}=q_{j, j+1}\left\langle n_{j}\left(1-n_{j+1}\right)\right\rangle$. In the mean-field approximation,

27 This can be seen easily from (F.8)-(F.10).

${ }^{28}$ We have to mention here that these results up to now do not include the case of parallel dynamics. 
$\left\langle n_{j}\left(1-n_{j+1}\right)\right\rangle=\left\langle n_{j}\right\rangle\left\langle\left(1-n_{j+1}\right)\right\rangle$ and, hence,

$$
J=J_{j, j+1}=q_{j, j+1} c_{j}\left(1-c_{j+1}\right)
$$

where $c_{j}=\left\langle n_{j}\right\rangle$. In order to calculate the steady-state flux $J$ as a function of the mean density $c$ of the particles, Tripathy and Barma [137] used two different iteration schemes based on the equation (G.1).

(i) Constant-current iteration scheme: In this scheme, for a given system length $L$ and a fixed flux $J=J_{0}$, one starts with some value of $c_{1}$ and, computes all the other $c_{j}(j>1)$ using the equation (G.1), i.e.,

$$
c_{j+1}=1-\frac{J_{0}}{q_{j, j+1} c_{j}}, \quad j=1,2, \ldots, L
$$

together with the periodic boundary condition $c_{j+L}=c_{j}$. If the iteration converges, i.e., one gets all the site densities in the physically acceptable range $[0,1]$, one accepts the average of these final site densities to be the global mean density of the particles corresponding to the flux $J_{0}$. (ii) Constant-density iteration scheme: In this scheme, for a given system length $L$ and fixed global average density $c$, one begins by assigning the site densities $0 \leq c_{j}(0) \leq 1$ to the lattice sites subject to the global constraint $\frac{1}{L} \sum_{j} c_{j}(0)=c$. Then, the site densities are updated in parallel according to

$$
c_{j}(t+1)=c_{j}(t)+J_{j-1, j}(t)-J_{j, j+1}(t), \quad j=1,2, \ldots, L
$$

which follows from the equation (G.1). It is straightforward to verify that the this iteration scheme keeps the average global density $c$ unchanged at every step of updating and hence the name. After sufficient number of iterations the set of densities converge to a set $\left\{c_{j}\right\}$ and the flux on each bond converge to the steady-state flux $J_{0}$.

\section{$\mathrm{H}$ Self-consistent equations for $v_{x}$ and $v_{y}$ in the mean-field approx- imation of the BML model}

Suppose, $v_{x}$ and $v_{y}$ denote the average speeds of east-bound and north-bound vehicles, respectively. Then, on the average, an east-bound vehicle spends a time $1 / v_{x}$ at a site whereas a north-bound vehicle spends a time $1 / v_{y}$ at a site. The north-bound vehicles lead to a reduction of the speed of the eastbound vehicles by $n_{y} / v_{y}$. Moreover, because of the hindrance of the east-bound vehicles by other east-bound vehicles ahead of it there will be further reduction

of the speed of the east-bound vehicles by $n_{x}\left[\frac{1}{v_{x}}-1\right]$. Furthermore, if the density 
of the overpasses is $f_{o}$, then

$$
v_{x}=1-\left(1-f_{o}\right)\left[\frac{n_{y}}{v_{y}}+n_{x}\left(\frac{1}{v_{x}}-1\right)\right]
$$

Similarly, the corresponding equation for $v_{y}$ is given by

$$
v_{y}=1-\left(1-f_{o}\right)\left[\frac{n_{x}}{v_{x}}+n_{y}\left(\frac{1}{v_{y}}-1\right)\right]
$$

In the special case $n_{x}=n_{y}=n / 2$ both the equations (H.1) and (H.2) reduce to the form

$$
v=1-\left(1-f_{o}\right)\left[\frac{1}{v}-\frac{1}{2}\right] n
$$

where $v_{x}=v_{y}=v$. The solution of the quadratic equation (H.3) for $v$ is

$$
v=\frac{1}{2}\left[1+\frac{1-f_{o}}{2} n+\sqrt{\left(1+\frac{1-f_{o}}{2} n\right)^{2}-4\left(1-f_{o}\right) n}\right]
$$

\section{Derivation of the equations in the microscopic theory of the BML model}

By definition, $n_{\uparrow}(x, y ; t)\left(n_{\rightarrow}(x, y: t)\right)$ is unity if the site $(x, y)$ is occupied at time $t$ by a north-bound (east-bound) vehicle and zero if the site $(x, y)$ is not occupied by a north-bound (east-bound) vehicle. Normalization requires $n_{\uparrow}(x, y ; t)+n_{\rightarrow}(x, y ; t)=1-n_{\text {empty }}(x, y ; t)$ where the two-state variable $n_{\text {empty }}(x, y ; t)$ is unity if, at time $t$, the site $(x, y)$ is empty and zero if the site $(x, y)$ is not empty. In order to describe the state of the signals at time $t$, one also defines a two-state variable $S(t): S(t)=1(0)$ if the signal is green (red) for the vehicles under consideration. The space-average of $n_{\uparrow}(x, y ; t)$ and $n_{\rightarrow}(x, y ; t)$ are $c_{\rightarrow}(t)$ and $c_{\uparrow}(t)$, respectively. Besides, the time-average of $S(t)$ is $1 / 2$.

The updating rules of the BML model lead to the equations

$$
\begin{aligned}
& n_{\rightarrow}(x, y ; t+1)=n_{\rightarrow}(x, y ; t)\left[n_{\uparrow}(x+1, y ; t)+n_{\rightarrow}(x+1, y ; t)\right] S(t) \\
& +n_{\rightarrow}(x-1, y ; t)\left[1-n_{\uparrow}(x, y ; t)-n_{\rightarrow}(x, y ; t)\right] S(t)+n_{\rightarrow}(x, y ; t)[1-S(t)]
\end{aligned}
$$

The first term on the right hand side of equation (I.1) describes the situation when the east-bound vehicle, which was at the site $(x, y)$ at time $t$, finds a 
green signal but cannot move because the next site towards east is occupied by another vehicle. The second term on the right hand side of (I.1) corresponds to the situation where the east-bound vehicle, which was at the site $(x-1, y)$ at time $t$, finds a green signal and moves to the next site towards east, which was empty. The last term on the right hand side of equation (I.1) arises from the possibility that the east-bound vehicle, which was at $(x, y)$ at time $t$, could not move because of a red signal, irrespective of the state of occupation of the next site towards east. Following the similar arguments, one can also write down the corresponding equation for the north-bound vehicles. 


\section{References}

[1] S.M. de Oliveira, P.M.C. de Oliveira and D. Stauffer Evolution, Money, War and Computers (Teubner, 1999); F. Schweitzer (ed.), Self-organization of Complex Systems: From Individual to Collective Dynamics (Gordon and Breach, 1997); G. Weisbuch, Complex Systems Dynamics (Addison-Wesley, 1990)

[2] H. Reiss, A.D. Hammerich and E.W. Montroll, J. Stat. Phys. 42, 647 (1986)

[3] D.E. Wolf, M. Schreckenberg and A. Bachem (eds.), Traffic and Granular Flow (World Scientific, Singapore, 1996)

[4] M. Schreckenberg and D.E. Wolf (eds.), Traffic and Granular Flow '97 (Springer, Singapore, 1998)

[5] V.J. Pande, A. Yu Grossberg and T. Tanaka, to appear in Rev. Mod. Phys. (2000)

[6] E. Ben-Jacob, Contemp. Phys. 34, 247 (1993); 38, 205 (1997)

[7] S. Kauffman, At Home in the Universe (Viking, 1995)

[8] P.W. Anderson, K.J. Arrow and D. Pines, The Economy as an Evolving Complex System (Addison-Wesley, 1988); W.B. Arthur, S. Durlauf and D. Lane, The Economy as an Complex System II (Addison-Wesley, 1997); J. Kertész and I. Kondor, Econophysics: An Emerging Science (Kluwer, 1998); J.P. Bouchaud, P. Cizeau, L. Laloux and M. Potters, Phys. World, Jan. 1999, p.25.; J.-P. Bouchand and M. Potters, Theory of Financial Risk (Cambridge University Press, to be published)

[9] G. Parisi, Physica A 263, 557 (1999)

[10] R. Herman and K. Gardels, Sci. Am. 209(6), 35 (1963)

[11] D.C. Gazis, Science, 157, 273 (1967)

[12] I. Prigogine and R. Herman, Kinetic Theory of Vehicular Traffic (Elsevier, Amsterdam, 1971)

[13] D. Helbing, Verkehrsdynamik: Neue Physikalische Modellierungskonzepte (in German) (Springer, 1997)

[14] B. Schmittmann and R.K.P. Zia, in: Phase Transitions and Critical Phenomena, Vol. 17, eds. C. Domb and J.L. Lebowitz (Academic Press, 1995); Phys. Rep. 301, 45 (1998); R.K.P. Zia, L.B. Shaw, B. Schmittmann and R.J. Astalos, cond-mat/9906376

[15] G. Schütz, in: Phase Transitions and Critical Phenomena, eds. C. Domb and J.L. Lebowitz (to appear)

[16] H. Spohn, Large Scale Dynamics of Interacting Particles (Springer, 1991) 
[17] V. Privman (ed.), Nonequilibrium Statistical Mechanics in One Dimension (Cambridge University Press, 1997)

[18] S. Wolfram, Theory and Applications of Cellular Automata, (World Scientific, 1986); Cellular Automata and Complexity (Addison-Wesley, 1994).

[19] K. Nagel, J. Esser and M. Rickert, in: Annu. Rev. Comp. Phys., ed. D. Stauffer (World Scientific, 1999)

[20] K. Nagel and M. Schreckenberg, J. Physique I, 2, 2221 (1992); K. Nagel, in: Physics Computing '92, eds. R.A. de Groot and J. Nadrchal (World Scientific, 1993), p.419; K. Nagel, High Speed Microsimulations of Traffic Flow, Ph.D Thesis, University of Cologne (1995).

[21] O. Biham, A.A. Middleton and D. Levine, Phys. Rev. A 46, R6124 (1992)

[22] B. Derrida and M.R. Evans, in: Nonequilibrium Statistical Mechanics in One Dimension, ed. V. Privman (Cambridge University Press, 1997); B. Derrida, Phys. Rep. 301, 65 (1998)

[23] G. Schütz, R. Ramaswamy and M. Barma, J. Phys. A 29, 837 (1996)

[24] H.E. Stanley, Introduction to Phase Transitions and Critical Phenomena (Oxford University Press, 1971)

[25] N. Goldenfeld, Lectures on Phase Transitions and Renormalization Group (Addision Wesley, 1992)

[26] P. Bak, How Nature Works: The Science of Self-Organized Criticality (Copernicus, New York, 1996)

[27] D. Dhar, Physica A 263, 4 (1999) and references therein

[28] J.D. Gunton and M. Droz, Lect. Notes in Phys. Vol. 183 (Springer, 1983); A. Bray, Adv. Phys. 43, 357 (1994)

[29] A.D. May, Traffic Flow Fundamentals (Prentice-Hall, 1990)

[30] B.D. Greenshields, Proc. Highw. Res. 14, 448 (1935)

[31] H. Greenberg, Op. Res. 7, 79 (1959)

[32] F.L. Hall, in [3], p. 11

[33] Th. Winzer, Messung von Beschleunigungsverteilungen (Measurements of Acceleration Distributions), Forschung Straßenbau und Straßentechnik (Research in Road Construction and Traffic Engineering), Federal Ministry of Transportation, Bonn, Germany (1980)

[34] D H. Hoefs, Untersuchung des Fahrverhaltens in Fahrzeugkolonnen, Straßenbau und Verkehrstechnik, Heft 8, Federal Ministry of Transportation, Bonn, Germany (1972)

[35] C.F. Daganzo, M.J. Cassidy, R.L. Bertini, Transp. Res. A 33, 365 (1999) 
[36] J. Treiterer: Ohio State Technical Report No. PB 246 094, (1975)

[37] B.S. Kerner and H. Rehborn, Phys. Rev. E 53, R1297 (1996)

[38] B.S. Kerner and H. Rehborn, Phys. Rev. E 53, R4275 (1996)

[39] B.S. Kerner, in [4], p. 239

[40] F.L. Hall, B.L. Allen and M.A. Gunter, Transp. Res. A20, 197 (1986)

[41] L. Neubert, L. Santen, A. Schadschneider and M. Schreckenberg, Phys. Rev. E 60, 6480 (1999)

[42] B.S. Kerner and H. Rehborn, Phys. Rev. Lett. 79, 4030 (1998)

[43] B.S. Kerner, Phys. Rev. Lett. 81, 3797 (1998)

[44] B.S. Kerner, Phys. World 8, 25 (1999)

[45] M. Koshi, M. Iwasaki, and I. Okuhra, in Proceedings of the 8th International Symposium on Transportation and Traffic Theory; edited by V.F. Hurdle, E. Hauer, and G.N. Steward (University of Toronto Press, Toronto, Ontario 1983), p. 403

[46] D. Helbing, Phys. Rev. E 55, 3735 (1997)

[47] D. Helbing, Phys. Rev. E 55, R25 (1997)

[48] P. Wagner, Z. Naturforsch. 52a, 600 (1997)

[49] R. Kühne, in: N. Gartner, C.J. Messner, A.J. Rathi (eds.), Transportation Research Board (TRB) Special Report 165, "Traffic Flow Theory," 2nd ed. (1998)

[50] M.J. Lighthill and G.B. Whitham, Proc. Roy. Soc. Lond. A 229, 281 (1955)

[51] G.B. Whitham, Linear and Nonlinear Waves (Wiley, 1974); Lectures on Wave Propagation (Springer, 1979)

[52] P.I. Richards, Op. Res. 4, 42 (1956)

[53] J.M. Burgers, The Nonlinear Diffusion Equation: Asymptotic Solutions and Statistical Problems (Reidel, Boston, 1974)

[54] M. Leibig, Phys. Rev. E 49, 184 (1994)

[55] H.J. Payne, in: Mathematical Models of Public Systems, ed. G.A. Bekey (Simulation Council, La Jolla, 1971), Vol. 1

[56] H.J. Payne, in: Research Directions in Computer Control of Urban Traffic Systems, ed. W.S. Levine, E. Lieberman and J.J. Fearnsides (American Society of Civil Engineers, New York, 1979)

[57] R.D. Kühne, in: Proc. 9th International Symposium on Transportation and Traffic Theory, ed. I. Volmuller and R. Hamerslag (VNU Science Press, Utrecht, 1984) 
[58] R.D. Kühne, in: Proc. 10th International Symposium on Transportation and Traffic Theory, ed. N.H. Gartner and N.H.M. Wilson (Elsevier, 1987)

[59] B.S. Kerner and P. Konhäuser, Phys. Rev. E 48, R2335 (1993); 50, 54 (1994); B.S. Kerner, P. Konhäuser and M. Schilke, Phys. Rev. E 51, 6243 (1995); B.S. Kerner, S.L. Klenov and P. Konhäuser, Phys. Rev. E 56, 4200 (1997).

[60] D.A. Kurtze and D.C. Hong, Phys. Rev. E 52, 218 (1995); D.C. Hong and S. Yue, Phys. Rev. E 58, 4763 (1998).

[61] M.Y. Choi and H.Y. Lee, Phys. Rev. E 52, 5979 (1995)

[62] H.Y. Lee, H.-W. Lee and D. Kim, Phys. Rev. Lett. 81, 1130 (1998); Phys. Rev. E 59, 5101 (1999)

[63] H.Y. Lee, D. Kim and M.Y. Choi, in [4], p. 433

[64] T. Nagatani, Phys. Rev. E 59, 4857 (1999)

[65] D. Helbing and M. Treiber, Science 282, 2002 (1998)

[66] D. Helbing, and M. Treiber, Phys. Rev. Lett. 81, 3042 (1998)

[67] D. Helbing, A. Hennecke, and M. Treiber, Phys. Rev. Lett. 82, 4360 (1999)

[68] G.F. Newell, Transp. Res. B. 27B(4), 281 (1993); 27B(4), 289 (1993); 27B(4), 305 (1993).

[69] C.F. Daganzo, Transp. Res. B. 28B, 269 (1994); 29B, 79 (1995); 29B, 277 (1995).

[70] I. Prigogine and F.C. Andrews, Op. Res. 8, 789 (1960)

[71] S.L. Paveri-Fontana, Trans. Res. 9, 225 (1975)

[72] H. Lehmann, Phys. Rev. E 54, 6058 (1996)

[73] C. Wagner, C. Hoffmann, R. Sollacher, J. Wagenhuber and B. Schürmann, Phys. Rev. E 54, 5073 (1996)

[74] C. Wagner, Physica A 245, 124 (1997); J. Stat. Phys. 90, 1251 (1998)

[75] D. Helbing, Phys. Rev. E 53, 2366 (1996); Phys. Rev. E 57, 6176 (1997); D. Helbing and M. Treiber, Phys. Rev. Lett. 81, 3042 (1998)

[76] T. Nagatani, Physica A 237, 67 (1997); J. Phys. Soc. Jap.66, 1219 (1997)

[77] K. Huang, Statistical Mechanics (2nd edition) (John Wiley, 1987)

[78] T. Nagatani, Physica A 237, 67 (1997)

[79] D. Helbing and A. Greiner, Phys. Rev. E 55, 5498 (1997); V. Shvetsov and D. Helbing, Phys. Rev. E 59, 6328 (1999); D. Helbing, Physica A 242, 175 (1997)

[80] T. Nagatani, J. Phys. Soc. Jap. 65, 3150 (1996) 
[81] R.W. Rothery, in: N. Gartner, C.J. Messner, A.J. Rathi (eds.), Transportation Research Board (TRB) Special Report 165, "Traffic Flow Theory," 2nd ed. (1998)

[82] D. Helbing and B. Tilch, Phys. Rev. 58, 133 (1998)

[83] A. Reuschel, Österreichisches Ingenieur-Archiv, 4, 193 (1950); A. Reuschel and Z. Öster, Ing. und Arch. Vereines, 95, 59 (1950)

[84] L.A. Pipes, J. Appl. Phys. 24, 274 (1953)

[85] R.E. Chandler, R. Herman and E.W. Montroll, Op. Res. 6, 165 (1958)

[86] D.C. Gazis, R. Herman and R.B. Potts, Op. Res. 7(4), 499 (1959)

[87] D.C. Gazis, R. Herman and R.W. Rothery, Op. Res. 9, 454 (1961)

[88] P.G. Gipps, Transp. Res. 15B, 105 (1981)

[89] M. Bando, K. Hasebe, A. Nakayama, A. Shibata and Y. Sugiyama, Phys. Rev. E 51, 1035 (1995); Jpn. J. Ind. Appl. Math. 11, 202 (1994)

[90] M. Bando, K. Hasebe, K. Nakanishi, A. Nakayama, A. Shibata, Y. Sugiyama, J. Physique I 5, 1389 (1995)

[91] Y. Sugiyama and H. Yamada, Phys. Rev. E 55, 7749 (1997)

[92] Y. Sugiyama and H. Yamada, in [4], p. 301

[93] T.S. Komatsu and S. Sasa, Phys. Rev. E 52, 5574 (1995)

[94] T. Nagatani, J. Phys. Soc. Jap. 65, 2333 (1996)

[95] T. Nagatani, Physica A 246, 460 (1997); 248, 353 (1998); Phys. Rev. E 58, $4271(1998)$

[96] K. Nakanishi, K. Itoh, Y. Igarashi and M. Bando, Phys. Rev. E 55, 6519 (1997)

[97] A.D. Mason and A.W. Woods, Phys. Rev. E 55, 2203 (1997)

[98] T. Nagatani and K. Nakanishi, Phys. Rev. E 57, 6415 (1998)

[99] M. Muramatsu and T. Nagatani, Phys. Rev. E 60, 180 (1999)

[100] H. Hayakawa and K. Nakanishi, Phys. Rev. E 57, 3839 (1998)

[101] C. Wagner, Physica A 260, 218 (1998)

[102] H. Lenz, C.K. Wagner and R. Sollacher, Eur. Phys. J. B 7, 331 (1999)

[103] N. Mitarai and H. Nakanishi, J. Phys. Soc. Jap. 68, 2475 (1999)

[104] T. Nagatani, K. Nakanishi and H. Emmerich, J. Phys. A 31, 5431 (1998)

[105] T. Nagatani, Physica A 265, 297 (1999)

[106] R. Mahnke and N. Pieret, Phys. Rev. E 56, 2666 (1997) 
[107] R. Mahnke and J. Kapusz, Phys. Rev. E 59, 117 (1999)

[108] K. Kaneko (ed.), Theory and Applications of Coupled Map Lattices (John Wiley and Sons, 1993) and the references therein

[109] S. Yukawa and M. Kikuchi, J. Phys. Soc. Jap. 64, 35 (1995)

[110] S. Yukawa and M. Kikuchi, J. Phys. Soc. Jap.65, 916 (1996)

[111] S. Yukawa and M. Kikuchi, in [4], p. 319

[112] S. Tadaki, M. Kikuchi, Y. Sugiyama and S. Yukawa, J. Phys. Soc. Jap.67, 2270 (1998) and in [4], p. 373

[113] K. Nagel and H.J. Herrmann, Physica A 199, 254 (1993)

[114] G. Sauermann and H.J. Herrmann, in [4], p. 481

[115] S. Krauss, P. Wagner and C. Gawron, Phys. Rev. E 54, 3707 (1996); 55, 5597 (1997)

[116] S. Krauss, in: [4], p. 269; see also S. Krauss, Microscopic Modeling of Traffic Flow: Investigation of Collision Free Vehicle Dynamics, DLRForschungsbericht 98-08

[117] J.G. Brankov, V.B. Priezzhev, A. Schadschneider and M. Schreckenberg, J. Phys. A 29, L229 (1996)

[118] P.W. Kasteleyn, J. Math. Phys. 4, 287 (1963)

[119] J.G. Brankov and M. Schreckenberg, J. Phys. A 31, 2133 (1998)

[120] M. Fukui and Y. Ishibashi, J. Phys. Soc. Jap. 65, 1868 (1996)

[121] S. Migowsky, T. Wanschura and P. Rujan, Z. Phys. B 95, 407 (1994); T. Wanschura, S. Migowsky and P. Rujan, in [3], p. 211

[122] E.F. Codd, Cellular Automata (Academic Press, 1968)

[123] For an amusing introduction see, for example, M. Gardner, Sci. Am. 220(4), $120(1970)$

[124] D. Stauffer, J. Phys. A 24, 909 (1991)

[125] K. Preston and M. Duff, Modern Cellular Automata: Theory and Applications (Plenum, 1984)

[126] B. Chopard and M. Droz, Cellular Automata Modelling of Physical Systems (Cambridge University Press, 1998)

[127] J. Marro and R. Dickman, Nonequilibrium Phase Transitions in Lattice Models (Cambridge University Press, 1999)

[128] M. Cremer and J. Ludwig, Mathematics and Computers in Simulation 28, 297 (1986) 
[129] A. Awazu, Phys. Lett. A 261, 309 (1999)

[130] N. Boccara, H. Fuks and Q. Zeng, J. Phys. A 30, 3329 (1997)

[131] D.W. Huang, J. Phys. A 31, 6167 (1998)

[132] K. Kawasaki, Phys. Rev. 145, 224 (1966); see also in: Phase Transitions and Critical Phenomena, Vol. 5A, eds. C. Domb and M.S. Green (Academic Press, 1976)

[133] S. Katz, J.L. Lebowitz and H. Spohn, Phys. Rev. B, 28, 1655 (1983); J. Stat. Phys. 34, 497 (1984)

[134] P.M. Binder, M. Paczuski and M. Barma, Phys. Rev. E 49, 1174 (1994)

[135] A.-L. Barabasi and H.E. Stanley, Fractal Concepts in Surface Growth (Cambridge University Press, 1995); T. Halpin-Healy and Y.-C. Zhang, Phys. Rep. 254, 215 (1995)

[136] J. Krug and H. Spohn, Phys. Rev. A 38, 4271 (1988); also in: Solids far from Equilibrium: Growth, Morphology and Defects, ed. C. Godreche (Cambridge University Press, 1991)

[137] G. Tripathy and M. Barma, Phys. Rev. Lett. 78, 3039 (1997); Phys. Rev. E 58, 1911 (1998); see also G. Tripathy, Ph.D. thesis, TIFR/University of Mumbai (1998), unpublished

[138] K. Nagel, Phys. Rev. E 53, 4655 (1996)

[139] M. Sasvári and J. Kertész, Phys. Rev. E 56, 4104 (1997)

[140] M. Schreckenberg, A. Schadschneider, K. Nagel and N. Ito, Phys. Rev. E 51, 2939 (1995); A. Schadschneider and M. Schreckenberg, J. Phys. A 26, L679 (1993)

[141] E.F. Moore, Proc. Symb. Appl. Math. 14, 17 (1962)

[142] A. Schadschneider and M. Schreckenberg, J. Phys. A 31, L225 (1998)

[143] A. Schadschneider, Eur. Phys. J. B 10, 573 (1999)

[144] H.A. Gutowitz, D. Victor and B.W. Knight, Physica 28D, 18 (1987)

[145] B.H. Wang and P.M. Hui, J. Phys. Soc. Jap. 66, 1238 (1997)

[146] A. Schadschneider and M. Schreckenberg, J. Phys. A 30, L69 (1997).

[147] D. ben-Avraham, in [17]

[148] L.C.Q. Vilar and A.M.C. De Souza, Physica A 211, 84 (1994)

[149] B. Eisenblätter, L. Santen, A. Schadschneider and M. Schreckenberg, Phys. Rev. E 57, 1309 (1998)

[150] M. Gerwinski and J. Krug, Phys. Rev. E 60, 188 (1999) 
[151] K. Nagel, Int. J. Mod. Phys. C 5, 567 (1994)

[152] G. Csányi and J. Kertész, J. Phys. A28, L427 (1995) (E: A29, 471 (1996))

[153] D. Chowdhury, A. Majumdar, K. Ghosh, S. Sinha and R.B. Stinchcombe , Physica A 246, 471 (1997).

[154] D. Chowdhury, A. Pasupathy and S. Sinha, Eur. Phys. J. B 5, 781 (1998)

[155] D. Chowdhury, L. Santen, A. Schadschneider, S. Sinha and A. Pasupathy, J. Phys. A 32, 3229 (1999)

[156] A. Schadschneider, in [4], p. 417

[157] K. Ghosh, A. Majumdar and D. Chowdhury, Phys. Rev. E 58, 4012 (1998)

[158] S. Cheybani, J. Kertész and M. Schreckenberg, J. Phys. A 31, 9787 (1998)

[159] L. Neubert, H.Y. Lee and M. Schreckenberg, J. Phys. A 32, 6517 (1999)

[160] S. Lübeck, M. Schreckenberg and K.D. Usadel, Phys. Rev. E 57, 1171 (1998)

[161] L. Roters, S. Lübeck and K.D. Usadel, Phys. Rev. E 59, 2672 (1999)

[162] J. Krug, Phys. Rev. Lett. 67, 1882 (1991)

[163] M. Henkel, G. Schütz, Physica A 206, 187 (1994)

[164] M.R. Evans, N. Rajewsky and E.R. Speer, J. Stat. Phys. 95, 45 (1999)

[165] J. de Gier and B. Nienhuis, Phys. Rev. E 59, 4899 (1999)

[166] N. Rajewsky, L. Santen, A. Schadschneider and M. Schreckenberg, J. Stat. Phys. 92, 151 (1998)

[167] B. Derrida, M.R. Evans, V. Hakim and V. Pasquier, J. Phys. A 26, 1493 (1993)

[168] G. Schütz and E. Domany, J. Stat. Phys.72, 277 (1993)

[169] N. Rajewsky, A. Schadschneider and M. Schreckenberg, J. Phys. A 29, L305 (1996)

[170] J.G. Brankov, N. Pesheva and N. Valkov, to appear in Phys. Rev. E; N.C. Pesheva, D.P. Daneva and J.G. Brankov, Rep. Math. Phys. 44, 1 (1999)

[171] G. Schütz, Phys. Rev. E 47, 4265 (1993)

[172] H. Hinrichsen, J. Phys. A 29, 3659 (1996)

[173] A. Honecker and I. Peschel, J. Stat. Phys. 88, 319 (1997)

[174] B. Derrida, in Statphys 19, Ed. Hao Bailin (World Scientific, 1996)

[175] A.B. Kolomeisky, G. Schütz, E.B. Kolomeisky and J.P. Straley, J. Phys. A 31, $6911(1998)$

[176] L.G. Tilstra and M.H. Ernst, J. Phys. A 31, 5033 (1998) 
[177] V. Popkov and G. Schütz, Europhys. Lett. 48, 257 (1999)

[178] V. Popkov, L. Santen, A. Schadschneider and G. Schütz, preprint (1999)

[179] K. Nagel and M. Paczuski, Phys. Rev. E 51, 2909 (1995); see also M. Paczuski and K. Nagel, in [3], p. 73

[180] T. Musha and H. Higuchi, Jap. J. Appl. Phys. 15, 1271 (1976); 17, 811 (1978)

[181] W. Feller, An Introduction to Probability Theory and Its Applications, Vol. 1 (Wiley, 1968)

[182] G.H. Weiss, Aspects and Applications of Random Walk (North-Holland, 1994)

[183] B.B. Mandelbrot, The Fractal Geometry of Nature (W.H. Freeman and Co. 1983)

[184] M. Takayasu and H. Takayasu, Fractals, 1, 860 (1993)

[185] A. Schadschneider and M. Schreckenberg, Ann. der Phys. 6, 541 (1997).

[186] M. Fukui and Y. Ishibashi, J. Phys. Soc. Jap. 66, 385 (1997)

[187] S.C. Benjamin, N.F. Johnson and P.M. Hui, J. Phys. A 29, 3119 (1996)

[188] R. Barlovic, L. Santen, A. Schadschneider and M. Schreckenberg, Eur. Phys. J. B 5, 793 (1998)

[189] R. Barlovic, Diploma Thesis, Universität Duisburg (1998); R. Barlovic, L. Santen, A. Schadschneider and M. Schreckenberg, in preparation

[190] L. Santen, Numerical Investigations of Discrete Models for Traffic Flow, Ph.D. thesis, Universität zu Köln (1999)

[191] H. Greenberg, A. Daou, Op. Res. 8, 524 (1960)

[192] T. Nagatani, J. Phys. A 26, L781 (1993); Physica A 202, 449 (1994); J. Phys. Soc. Jpn. 63, 52 (1994); J. Phys. A 29, 6531 (1996)

[193] M. Rickert, K. Nagel, M. Schreckenberg and A. Latour, Physica A 231, 534 (1996)

[194] P. Wagner, K. Nagel and D.E. Wolf, Physica A 234, 687 (1997)

[195] D. Chowdhury, D. E. Wolf and M. Schreckenberg, Physica A 235 , 417 (1997)

[196] K. Nagel, D.E. Wolf, P. Wagner, and P. Simon, Phys. Rev. E 58, 1425 (1998)

[197] W. Knospe, L. Santen, A. Schadschneider and M. Schreckenberg, Physica A 265, 614 (1999)

[198] X. Zhang and G. Hu, Phys. Rev. E 52, 4664 (1995)

[199] A. Awazu, J. Phys. Soc. Jpn. 67, 1071 (1998) 
[200] U.

Sparmann,

Spurwechselvorgänge auf zweispurigen BAB-Richtungsfahrbahnen. Forschung Straßenbau und Straßenverkehrstechnik 263, Bundesministerium für Verkehr, Bonn-Bad Godesberg, Germany (1978)

[201] P. Simon and H.A. Gutowitz, Phys. Rev. E 57, 2441 (1998)

[202] H.W. Lee, V. Popkov and D. Kim, J. Phys. A 30, 8497 (1997)

[203] M.E. Fouladvand and H.-W. Lee, Phys. Rev. E 60, 6465 (1999); M.E. Fouladvand and A. Schadschneider, in preparation

[204] D. Ktitarev, D. Chowdhury and D.E. Wolf, J. Phys. A 30, L221 (1997)

[205] J. Krug and P. A. Ferrari, J. Phys. A 29, L465 (1996)

[206] M. R. Evans, Europhys. Lett. 36, 13 (1996); J. Phys. A 30, 5669 (1997)

[207] J. Krug, in ref. [4], p. 285

[208] K. Mallick, J. Phys. A 29, 5375 (1996)

[209] E. Ben-Naim, P.L. Krapivsky and S. Redner, Phys. Rev. E 50, 822 (1994); E. Ben-Naim and P.L. Krapivsky, Phys. Rev. E 56, 6680 (1997); J. Phys. A 31, 8073 (1998); Phys. Rev. E 59, 88 (1999)

[210] T. Nagatani, J. Phys. Soc. Jpn. 65, 3386 (1996)

[211] T. Nagatani, H. Emmerich and K. Nakanishi, Physica A 255, 158 (1998)

[212] D. Helbing and B.A. Huberman, Nature 396, 738 (1998)

[213] K.H. Chung and P.M. Hui, J. Phys. Soc. Jap. 63, 4338 (1994)

[214] S.A. Janowsky and J.L. Lebowitz, Phys. Rev. A 45, 618 (1992); J. Stat. Phys. 77, 35 (1994)

[215] S. Yukawa, M. Kikuchi and S. Tadaki, J. Phys. Soc. Jap. 63, 3609 (1994)

[216] G. Schütz, J. Stat. Phys. 71, 471 (1993)

[217] H. Hinrichsen and S. Sandow, J. Phys. A 30, 2745 (1997)

[218] S. Goldstein and E.R. Speer, Phys. Rev. E 58, 4226 (1998)

[219] W. Knospe, L. Santen, A. Schadschneider and M. Schreckenberg, in [4], p. 349

[220] Z. Csahok and T. Vicsek, J. Phys. A 27, L591 (1994)

[221] H. Emmerich and E. Rank, Physica A 216, 435 (1995)

[222] T. Nagatani, J. Phys. Soc. Jap. 66, 1928 (1997)

[223] G. Diedrich, L. Santen, A. Schadschneider and J. Zittartz, to appear in Int. J. Mod. Phys. C 
[224] B.H. Wang, Y.R. Kwong and P.M. Hui, Phys. Rev. E 57, 2568 (1998); Physica A 254, $122(1998)$

[225] B.H. Wang, L. Wang and P.M. Hui, J. Phys. Soc. Jap. 66, 3683 (1997)

[226] B.H. Wang, L. Wang, P.M. Hui and B. Hu, Phys. Rev. E 58, 2876 (1998)

[227] H. Fuks and N. Boccara, Int. J. Mod. Phys. C 9, 1 (1998)

[228] H. Fuks, Phys. Rev. E 60, 197 (1999)

[229] D.E. Wolf, Physica A 263, 438 (1999)

[230] J. Werth, K. Froese and D.E. Wolf, to be published

[231] H. Emmerich and E. Rank, Physica A 234, 676 (1997)

[232] L. Santen and A. Schadschneider, unpublished results (1997)

[233] D. Helbing and M. Schreckenberg, Phys. Rev. E 59, R2505 (1999)

[234] L. Santen and A. Schadschneider, in preparation

[235] T. Nagatani, Physica A 223, 137 (1996)

[236] T. Tokihiro, D. Takahashi, J. Matsukidaira and J. Satsuma, Phys. Rev. Lett. 76, 3247 (1996)

[237] K. Nishinari and D. Takahashi, J. Phys. A 31, 5439 (1998)

[238] K. Nishinari and D. Takahashi, J. Phys. A 32, 93 (1999)

[239] H. Emmerich, T. Nagatani and K. Nakanishi, Physica A 254, 548 (1998)

[240] T. Nagatani, H. Emmerich and K. Nakanishi, Physica A254, 158 (1998); H. Emmerich and B. Kahng, Physica A259, 81 (1998)

[241] M. Fukui and Y. Ishibashi, J. Phys. Soc. Jap. 65, 1871 (1996).

[242] S. Tadaki, Phys. Rev. E 54, 2409 (1996); J. Phys. Soc. Jap. 66, 514 (1997)

[243] D. Stauffer and A. Aharony, Introduction to Percolation Theory (Taylor and Francis, London 1994)

[244] S. Tadaki and M. Kikuchi, Phys. Rev. E 50, 4564 (1994); J. Phys. Soc. Jap. 64, $4504(1995)$

[245] H.S. Gupta and R.Ramaswamy, J. Phys. A 29, L547 (1996)

[246] T. Nagatani, J. Phys. Soc. Jap. 62, 2533 (1993)

[247] S. Mukherji and S.M. Bhattacharjee, Physica A 245, 534 (1997)

[248] T. Nagatani, J. Phys. Soc. Jap. 62, 2656(1993)

[249] B.H. Wang, Y.F. Woo and P.M. Hui, J.Phys.A29, L31 (1996) 
[250] Y. Ishibashi and M. Fukui, J. Phys. Soc. Jap.63, 2882 (1994); 65, 1878 (1996)

[251] H.F. Chau, P.M. Hui and Y.F. Woo, J. Phys. Soc. Jap. 64, 3570 (1995)

[252] Y. Shi, adap-org/9509003

[253] H.F. Chau, K.Y. Wan and K.K. Yan, Physica A 254, 117 (1998)

[254] H.F. Chau and K.Y. Wan, cond-mat/9905014

[255] J.M. Molera, F.C. Martinez, J.A. Cuesta and R. Brito, Phys. Rev. E, 51, 175 (1995)

[256] M. Kaulke and S. Trimper, J. Phys. A 28, 5445 (1995)

[257] M. Fukui, H. Oikawa and Y. Ishibashi, J. Phys. Soc. Jap. 65, 2514 (1996)

[258] T. Nagatani, Phys. Rev. E 48, 3290 (1993)

[259] B.H. Wang, Y.F. Woo and P.M. Hui, J. Phys. Soc. Jap. 65, 2345 (1996).

[260] K.H. Chung, P.M. Hui and G.Q. Gu, Phys. Rev. E 51, 772 (1995)

[261] T. Nagatani, J.Phys.A 26, L1015 (1993); J. Phys. Soc. Jap. 62, 1085 (1993)

[262] G.Q. Gu, K.H. Chung and P.M. Hui, Physica A 217, 339 (1995).

[263] M. Fukui and Y. Ishibashi, J. Phys. Soc. Jap. 62, 3841 (1993)

[264] T. Nagatani, Physica A 198, 108 (1993)

[265] J.A. Cuesta, F.C. Martines, J.M. Molera and A. Sanchez, Phys. Rev. E 48, R4175 (1993); F.C. Martines, J.A. Cuesta, J.M. Molera and R. Brito, Phys. Rev. E51, R835 (1995)

[266] T. Nagatani, J. Phys. Soc. Jap. 63, 1228 (1994)

[267] T. Nagatani, J. Phys. Soc. Jap. 64, 1421 (1995)

[268] T. Nagatani and T. Seno, Physica A 207, 574 (1994)

[269] Y. Ishibashi and M. Fukui, J. Phys. Soc. Jap. 65, 2793 (1996)

[270] J. Török and J. Kertész, Physica A 231, 515 (1996)

[271] L. G. Brunnet and S. Goncalves, Physica A 237, 59 (1997)

[272] T. Horiguchi and T. Sakakibara, Physica A 252, 388 (1998)

[273] T. Horiguchi and T. Sakakibara, Interdisc. Inf. Sc. 4, 39 (1998)

[274] J. Freund and T. Pöschel, Physica A 219, 95 (1995)

[275] B. Chopard, P.O. Luthi and P.A. Queloz, J. Phys. A 29, 2325 (1996)

[276] P.M. Simon and K. Nagel, Phys. Rev. E 58, 1286 (1998)

[277] D. Chowdhury and A. Schadschneider, Phys. Rev. E 59, R1311 (1999); 
[278] A. Schadschneider, D. Chowdhury, E. Brockfeld, K. Klauck, L. Santen and J. Zittartz, cond-mat/9911312; D. Chowdhury, K. Klauck, L. Santen, A. Schadschneider and J. Zittartz (to be published)

[279] J. Esser and M. Schreckenberg, Int. J. Mod. Phys.C 8, 1025 (1997).

[280] M. Rickert and K. Nagel, Int. J. Mod. Phys.C 8, 483 (1997); K. Nagel and C.L. Barrett, Int. J. Mod. Phys. C 8, 505 (1997)

[281] B. Chopard, A. Dupuis and P. Luthi, in ref. [4], p. 153

[282] W. Dieterich, P. Fulde and I. Peschel, Adv. Phys. 29, 527 (1980)

[283] S. Sandow, S. Trimper and D. Mukamel, Phys. Rev. B 51, 2805 (1995)

[284] B.H. Wang, Y.R. Kwong, P.M. Hui and B. Hu, Phys. Rev. E 60, 149 (1999)

[285] S. Sandow, Phys. Rev. E 50, 2660 (1994); F.H.L. Essler and V. Rittenberg, J. Phys. A 29, 3375 (1996); K. Mallick and S. Sandow, J. Phys. A 30, 4513 (1997); T. Sasamoto, S. Mori and M. Wadati, J. Phys. Soc. Jpn. 65, 2000 (1996)

[286] T. Sasamoto, J. Phys. A 32, 7109 (1999)

[287] V. Karimipour, Phys. Rev. E 59, 117 (1999), Europhys. Lett. 47, 304 (1999); M.E. Fouladvand and F. Jafarpour, J. Phys. A 32, 5845 (1999); M. Khorrami and V. Karimipour, cond-mat/9908037

[288] P.F. Arndt, T. Heinzel and V. Rittenberg, J. Phys. A 31, L45 (1998); J. Stat. Phys. 90, 783 (1998); J. Stat. Phys. 97, 373 (1999); P.F. Arndt, preprint (1999)

[289] M.R. Evans, Y. Kafri, H.M. Koduvely and D. Mukamel, Phys. Rev. Lett. 80, 425 (1998); Phys. Rev. E 58, 2764 (1998)

[290] L.-H. Gwa and H. Spohn, Phys. Rev. A 46, 844 (1992)

[291] H. A. Bethe, Z. Phys. 71, 205 (1931); for a pedagogical introduction, see e.g. Y. A. Izyumov and Y.N. Skryabin, Statistical Mechanics of Magnetically Ordered Systems (Consultants Bureau, 1988)

[292] D. Kim, Phys. Rev. E 52, 3512 (1995); J. Phys. A 30, 3817 (1997); U. Bilstein and B. Wehefritz, J. Phys. A 30, 4925 (1997); G. Albertini, S.R. Dahmen and B. Wehefritz, J. Phys. A 29, L369(1996)

[293] B. Derrida and J.L. Lebowitz, Phys. Rev. Lett. 80, 209 (1998)

[294] C. Appert and B. Derrida, J. Stat. Phys. 94, 1 (1999); D.-S. Lee and D. Kim, Phys. Rev. E 59, 6476 (1999)

[295] G.M. Schütz, J. Stat. Phys. 88, 427 (1997)

[296] G.M. Schütz, Int. J. Mod. Phys. B 11, 197 (1997)

[297] J.T. MacDonald, J.H. Gibbs and A.C. Pipkin, Biopolymers 6, 1 (1968); J.T. MacDonald and J.H. Gibbs, Biopolymers 7, 707 (1969) 
[298] H.H. Jaeger and S.R. Nagel, Science 255, 1523 (1992); H.H. Jaeger, S.R. Nagel and R.P. Behringer, Phys. Today 4, 32 (1996); H.J. Herrmann, Phys. World 11, 31 (1997); L.P. Kadanoff, Rev. Mod. Phys. 71, 435 (1999); P.G. de Gennes, Rev. Mod. Phys. 71, S374 (1999)

[299] H.J. Herrmann, in: Nonlinear Physics of Complex Systems: Current Status and Future Trends, eds. J. Parisi, S.C. Müller and W. Zimmermann, Lec. Notes in Phys., Vol. 476, 23 (Springer, 1996);

H. Hayakawa and K. Nakanishi, Prog. Theor. Phys. Suppl. 130, 57 (1998)

[300] K.L. Gavrilov, Phys. Rev. E 56, 4860 (1997)

[301] O. Moriyama, N. Kuroiwa, M. Matsushita and H. Hayakawa, Phys. Rev. Lett. 80, 2833 (1998)

[302] O.J. O'Loan, M.R. Evans and M.E. Cates, Europhys. Lett. 42, 137 (1998); Phys. Rev. E 58, 1404 (1998)

[303] D. Chowdhury and R. C. Desai, to appear in Eur. Phys. J. B

[304] Z. Toroczkai and R.K.P. Zia, Phys. Lett. A 217, 97 (1996); J. Stat. Phys. 87, $545(1997)$

[305] M. Rubinstein, Phys. Rev. Lett., 59, 1946 (1987); T.A.J. Duke, Phys. Rev. Lett., 62, 2877 (1989)

[306] For an elementary introduction to gel electrophoresis, see, for example, J.L. Viovy, T. Duke and F. Caron, Contemp. Phys. 33, 25 (1992)

[307] I. Csabai, J. Phys. A. 27, L417 (1994).

[308] M. Takayasu, H. Takayasu and T. Sato, Physica A 233, 824 (1996); M. Takayasu, A. Yu Tretyakov, K. Fukuda and H. Takayasu, in ref. [4], p. 57

[309] N. Vandewalle, D. Strivay, H.P. Garnir and M. Ausloos, in ref. [4], p. 81

[310] T. Ohira and R. Sawatari, Phys. Rev. E 58, 193 (1998)

[311] G. Le Caër, Physica A157, 669 (1989)

[312] B.A. Huberman and N.S. Glance, Proc. Natl. Acad. Sci. USA 90, 7716 (1993)

[313] M.Y. Choi and B.A. Huberman, Phys. Rev. B 28, 2547 (1983)

[314] B. Schönfisch and A. de Roos, BioSystems 51, 123 (1999)

[315] H.J. Blok and B. Bergersen, Phys. Rev. E 59, 3876 (1999)

[316] F. Spitzer, Adv. Math. 5, 246 (1970)

[317] T.M. Liggett, Interacting Particle Systems, (Springer, 1985)

[318] F.C. Alcaraz, M. Droz, M. Henkel, V. Rittenberg, Ann. Phys. 230, 250 (1994)

[319] K. Krebs and S. Sandow, J. Phys. A 30, 3165 (1997) 
[320] N. Rajewsky and M. Schreckenberg, Physica A 245, 139 (1997)

[321] K. Klauck and A. Schadschneider, Physica A 271, 102 (1999)

[322] A. Klümper, A. Schadschneider and J. Zittartz, J. Phys. A 24, L955 (1991); Z. Phys. B 87, 281 (1992); Europhys. Lett, 24293 (1993)

[323] R.B. Stinchcombe and G. Schütz, Europhys. Lett. 29, 663 (1995); Phys. Rev. Lett. 75, 140 (1995)

[324] G. Schütz, Eur. Phys. J. B 5, 589 (1998)

[325] T. Sasamoto and M. Wadati, J. Phys. Soc. Jpn. 66, 279 (1997) 